\title{
Chemistry and deposition in the Model of Atmospheric composition at Global and Regional scales using Inversion Techniques for Trace gas Emissions (MAGRITTE v1.1) - Part 1: Chemical mechanism
}

\author{
Jean-François Müller $^{1}$, Trissevgeni Stavrakou ${ }^{1}$, and Jozef Peeters ${ }^{2}$ \\ ${ }^{1}$ Royal Belgian Institute for Space Aeronomy, Avenue Circulaire 3, 1180 Brussels, Belgium \\ ${ }^{2}$ Department of Chemistry, University of Leuven, Celestijnenlaan 200F, 3001 Leuven, Belgium
}

Correspondence: Jean-François Müller (jean-francois.muller@ aeronomie.be)

Received: 9 December 2018 - Discussion started: 19 December 2018

Revised: 18 April 2019 - Accepted: 17 May 2019 - Published: 14 June 2019

\begin{abstract}
A new chemical mechanism for the oxidation of biogenic volatile organic compounds (BVOCs) is presented and implemented in the Model of Atmospheric composition at Global and Regional scales using Inversion Techniques for Trace gas Emissions (MAGRITTE v1.1). With a total of 105 organic species and over 265 gas-phase reactions, 69 photodissociations, and 7 heterogeneous reactions, the mechanism treats the chemical degradation of isoprene - its main focus - as well as acetaldehyde, acetone, methylbutenol, and the family of monoterpenes. Regarding isoprene, the mechanism incorporates a state-of-the-art representation of its oxidation scheme accounting for all major advances put forward in recent theoretical and laboratory studies. The recycling of $\mathrm{OH}$ radicals in isoprene oxidation through the isomerization of $Z$ - $\delta$-hydroxyperoxy radicals is found to enhance $\mathrm{OH}$ concentrations by up to $40 \%$ over western Amazonia in the boundary layer and by $10 \%-15 \%$ over the southeastern US and Siberia in July. The model and its chemical mechanism are evaluated against the suite of chemical measurements from the SEAC ${ }^{4} \mathrm{RS}$ (Studies of Emissions and Atmospheric Composition, Clouds and Climate Coupling by Regional Surveys) airborne campaign, demonstrating a good overall agreement for major isoprene oxidation products, although the aerosol hydrolysis of tertiary and non-tertiary nitrates remain poorly constrained. The comparisons for methylnitrate indicate a very low nitrate yield $\left(<3 \times 10^{-4}\right)$ in the $\mathrm{CH}_{3} \mathrm{O}_{2}+\mathrm{NO}$ reaction. The oxidation of isoprene, acetone, and acetaldehyde by $\mathrm{OH}$ is shown to be a substantial source of enols and keto-enols, primarily through the photolysis of multifunctional carbonyls generated in their oxidation schemes. Oxidation of those enols by $\mathrm{OH}$ radi-
\end{abstract}

cals constitutes a sizable source of carboxylic acids estimated at $9 \mathrm{Tg}(\mathrm{HC}(\mathrm{O}) \mathrm{OH}) \mathrm{yr}^{-1}$ and $11 \mathrm{Tg}\left(\mathrm{CH}_{3} \mathrm{C}(\mathrm{O}) \mathrm{OH}\right) \mathrm{yr}^{-1}$ or $\sim 20 \%$ of their global identified source. The ozonolysis of alkenes is found to be a smaller source of $\mathrm{HC}(\mathrm{O}) \mathrm{OH}$ $(6 \mathrm{Tg} \mathrm{HC} \mathrm{O}) \mathrm{OH} \mathrm{yr}^{-1}$ ) than previously estimated, due to several factors including the strong deposition sink of hydroxymethyl hydroperoxide (HMHP).

\section{Introduction}

The terrestrial biosphere is, by far, the largest source of nonmethane volatile organic compounds (NMVOCs) into the global atmosphere (Guenther et al., 2012). Because those biogenic VOCs (BVOCs) are generally very reactive, their chemical degradation takes place mostly in the boundary layer in the vicinity of the emission regions, where they have a strong impact on the budget of oxidants and the formation and growth of secondary organic aerosol (SOA), a major component of fine particulate matter (Seinfeld and Pandis, 2006; Hallquist et al., 2009). Even far away from those regions, longer-lived intermediates generated in their oxidation (e.g. organic nitrates and peroxynitrates) have a large impact on nitrogen oxides $\left(\mathrm{NO}_{x}\right)$, hydroxyl radical $(\mathrm{OH})$, and ozone levels (Paulot et al., 2012).

Among the BVOCs, isoprene has by far the largest global emissions, of the order of $500 \mathrm{Tg} \mathrm{yr}^{-1}$, representing about $50 \%$ of all BVOCs; other major biogenic compounds in terms of emissions include monoterpenes, methanol, acetone, acetaldehyde, and ethanol (Guenther et al., 2012). The 
complex chemical degradation mechanism and the profound impact of isoprene on air quality and the climate has been the topic of numerous field (Trainer et al., 1987; Claeys et al., 2004; Lelieveld et al., 2008; Hofzumahaus et al., 2009; Toon et al., 2016; Carlton et al., 2018; Mao et al., 2018), laboratory (Tuazon and Atkinson, 1989; Paulot et al., 2009a, b; Crounse et al., 2011; Wolfe et al., 2012; Kwan et al., 2012; Lin et al., 2013; Fuchs et al., 2013; Bates et al., 2014, 2016; Nguyen et al., 2015a, 2016; Schwantes et al., 2015; Teng et al., 2017; Novelli et al., 2018b; Berndt et al., 2019), theoretical (Peeters et al., 2009; Kjaergaard et al., 2012; Crounse et al., 2013; Peeters et al., 2014; Peeters and Nguyen, 2012; Liu et al., 2017; Praske et al., 2018; Møller et al., 2019) and modelling studies (Stavrakou et al., 2010; Paulot et al., 2012; Taraborrelli et al., 2012; Jenkin et al., 2015; Squire et al., 2015; Travis et al., 2016; Lelieveld et al., 2016; Silva et al., 2018; Stadtler et al., 2018; Mao et al., 2018; Li et al., 2018).

Our understanding of isoprene oxidation has expanded considerably in the last decade. Most importantly perhaps, the traditional views regarding the fate of large, multifunctional peroxy radicals formed in the oxidation of isoprene and other NMVOCs has been radically altered by the realization that $\mathrm{H}$-shift reactions in such radicals can sometimes be fast enough to compete with, or even outrun, their reactions with nitric oxide and peroxy radicals (Peeters et al., 2009; Crounse et al., 2011; Teng et al., 2017). The impact of the 1,6 H-shifts in allylic peroxy radicals formed in the oxidation of isoprene by $\mathrm{OH}$ is enhanced by their thermal instability allowing fast interconversion of the different peroxy isomers and conformers (Peeters et al., 2009), such that the $1,6 \mathrm{H}$-shifts can compete with the conventional bimolecular reactions for the entire pool of initial peroxys, which greatly affects the product yields (Peeters and Müller, 2010; Peeters et al., 2014; Teng et al., 2017). Other examples of peroxy isomerization reactions shown to be of importance include 1,4 aldehyde H-shifts (Asatryan et al., 2010; Crounse et al., 2012) and the very fast enol-H-shifts (Peeters and Nguyen, 2012) as well as hydroperoxide H-shifts (Jørgensen et al., 2016). The resulting autoxidation reactions generate multifunctional hydroperoxides shown in some cases (in monoterpene oxidation) to be of such extremely low volatility as to play a crucial role in SOA and cloud condensation nuclei (CCN) formation (Crounse et al., 2013; Jokinen et al., 2014, 2015), while in other cases, they are believed to be an important source of $\mathrm{HO}_{x}$ radicals through photodissociation (Peeters and Müller, 2010; Wolfe et al., 2012; Liu et al., 2017, 2018). The recycling of $\mathrm{HO}_{x}$ radicals associated with peroxy $\mathrm{H}$-shifts and their subsequent reactions, as well as with other previously unsuspected reactions such as epoxide formation from activated hydroxy hydroperoxy radicals (Paulot et al., 2009a), has led to a reassessment of the overall impact of isoprene (and other BVOCs) on $\mathrm{OH}$ and $\mathrm{HO}_{2}$ levels, now found to be fairly consistent with $\mathrm{HO}_{x}$ measurements in isoprene photooxidation experiments (Fuchs et al., 2013; Novelli et al., 2018b) as well as in field experiments in isoprene-rich, low- $\mathrm{NO}_{x}$ environments (Bottorff et al., 2018). The importance of isoprene-derived epoxides stems from their major role as precursors of SOA demonstrated by laboratory and field measurements (Paulot et al., 2009a; Surratt et al., 2010; Lin et al., 2012, 2013). Finally, the impact of isoprene on $\mathrm{NO}_{x}$ levels has also been reevaluated, due to a better assessment of organic nitrate formation in isoprene oxidation by OH (Paulot et al., 2009b; Lee et al., 2014; Teng et al., 2017; Wennberg et al., 2018) and $\mathrm{NO}_{3}$ (Kwan et al., 2012; Schwantes et al., 2015; Wennberg et al., 2018), as well as of the balance between $\mathrm{NO}_{x}$-recycling pathways such as photolysis (Müller et al., 2014) and $\mathrm{NO}_{x}$ terminal losses through heterogeneous hydrolysis in aqueous aerosols (Romer et al., 2016) and dry deposition (Nguyen et al., 2015b).

A proper model assessment of the role of BVOCs in the global troposphere and in issues such as air quality and the interaction between the biosphere, the atmosphere and the climate requires the implementation of up-to-date, state-of-the-art chemical mechanisms in large-scale (global or regional) models. Whereas completely explicit mechanisms are not advisable due to computational cost concerns, oversimplified mechanisms are clearly not appropriate as tools to explore the impact of mechanistic changes, especially in the context of the rapid evolution of our understanding of the mechanisms. Here, we present a semiexplicit mechanism of intermediate complexity incorporating the major advances reported above. It covers the oxidation of isoprene, monoterpenes, methanol, acetone, acetaldehyde, ethanol, and 2-methyl-3-butene-2-ol (abbreviated as methylbutenol or MBO). This mechanism is implemented in the Model of Atmospheric composition at Global and Regional scales using Inversion Techniques for Trace gas Emissions (MAGRITTE v1.1), which is based on the previous global model IMAGES (Muller and Brasseur, 1995; Stavrakou et al., 2009a, b, 2015; Bauwens et al., 2016).

Given the very large uncertainties in monoterpene oxidation, their treatment is still very crude in the mechanism, the focus being put on the formation yield of important products. Regarding isoprene, the mechanism relies on the Leuven Isoprene Mechanism (Peeters et al., 2009, 2014), on the extensive, explicit Caltech oxidation mechanism (ca. 900 reactions and 400 species) recently presented by Wennberg et al. (2018), based on a critical appraisal of the relevant theoretical and laboratory studies, and on the very recent experimental investigation of Berndt et al. (2019). For other reactions not addressed in those studies, it also relies on the Master Chemical Mechanism (MCM) (Saunders et al., 2003; Jenkin et al., 2015) and on our own evaluation. The mechanism also incorporates important new mechanistic developments related to, e.g. the revisited role of hydroperoxycarbonyl photolysis (Liu et al., 2017, 2018) and the fate of enols and keto-enols produced from such processes. Due to these developments, the oxidation of isoprene, as well as of other compounds (e.g. acetone and acetaldehyde), by $\mathrm{OH}$ entails a previously unsuspected source of formic and acetic acid, 
for which atmospheric observations suggest the existence of large missing sources (Paulot et al., 2011; Stavrakou et al., 2012; Millet et al., 2015), especially since the $\mathrm{HC}(\mathrm{O}) \mathrm{OH}$ source due to alkene ozonolysis through the Criegee Intermediate $\mathrm{CH}_{2} \mathrm{OO}$ recently turned out smaller than previously thought (Sheps et al., 2017; Allen et al., 2018).

The complete chemical mechanism of BVOC oxidation is presented in Sect. 2. The parameterization of Henry's law constants and dry deposition velocities is presented and evaluated in a companion paper (Müller et al., 2018). Simulations with the MAGRITTE model and the updated chemical mechanism are presented in Sect. 4, including an evaluation against airborne measurements over the eastern United States (Sect. 4.3) and a presentation of the global sources of carboxylic acids (Sect. 4.4) and glyoxal (Sect. 4.5) resulting from the implementation of the chemical mechanism.

\section{The chemical mechanism of BVOC oxidation in MAGRITTE}

The list of chemical species and the complete gas-phase BVOC oxidation mechanism are given in Tables 1-3.

\subsection{Isoprene $+\mathrm{OH}$}

\subsubsection{Initial steps of the mechanism}

To limit the number of species and reactions, the $\mathrm{OH}$-adducts formed from the reaction of isoprene with $\mathrm{OH}$ are not explicitly represented and the isoprene peroxys are lumped into three compounds: ISOPBO2 and ISODO2 resulting from the addition of $\mathrm{OH}$ to carbons 1 and $4\left(\mathrm{C}_{1}\right.$ and $\left.\mathrm{C}_{4}\right)$, respectively, and ISOPEO 2 resulting from $\mathrm{OH}$ addition to the central carbons (see Peeters et al., 2014, regarding carbon numbering). For example, ISOPBO2 includes the 1,2-OH-peroxy as well as the 1,4-OH-peroxy, which can undergo a 1,6 H-shift leading to a $\delta$-hydroperoxyaldehyde (HPALD1) and other products. The ratio of $\mathrm{OH}$ addition to $\mathrm{C}_{4}$ to addition to $\mathrm{C}_{1}$ is $37: 63$ (Wennberg et al., 2018). Based on a detailed steadystate analysis, the bulk isomerization rate of ISOPBO2 and ISOPDO 2 was shown to increase linearly with the sink rate $\left(k_{\mathrm{p}}\right)$ of the traditional peroxy reactions (Peeters et al., 2014). The reason for this behaviour is that at low $k_{\mathrm{p}}$, the ratio of the $Z-\delta$-OH-peroxys over the lower-energy $\beta$-OH-peroxys is close to their equilibrium ratio, of the order of only $\sim 0.01$, whereas at the high $k_{\mathrm{p}}$ limit, where all peroxys have a similar lifetime, their ratio is governed by their initial formation branching ratio, which is an order of magnitude higher (Peeters et al., 2014; Teng et al., 2017). The following expressions of the bulk 1,6 isomerization rates are obtained by linear regression of the bulk rates between 285 and $305 \mathrm{~K}$, based on the experimental estimates of the peroxy unimolec- ular reaction rates (Teng et al., 2017; Wennberg et al., 2018).

$$
\begin{aligned}
k_{\text {ISOPBO } 2}^{1,6}= & 3.409 \times 10^{12} \cdot \exp (-10698 / T)+ \\
& k_{\mathrm{p}} \cdot 1.07 \times 10^{-3} \cdot \exp (64 / T) \\
k_{\text {ISOPDO2 }}^{1,6}= & 4.253 \times 10^{8} \cdot \exp (-7254 / T)+ \\
& k_{\mathrm{p}} \cdot 2.33 \times 10^{-7} \cdot \exp (3662 / T)
\end{aligned}
$$

The steady-state $Z$ - $\delta$-OH-peroxy / $\beta$-OH-peroxy ratio is essentially always established in the atmosphere and remains constant in time at given temperature and $\mathrm{NO} / \mathrm{HO}_{2}$ levels, as implied in our approach to representing the bulk peroxy isomerization rate. Note that the steady-state ratio used here, based on the $\mathrm{RO}_{2}$ kinetic coefficients of Teng et al. (2017), differs only slightly from the ratio based on the kinetic coefficients of LIM1 (Peeters et al., 2014) and MCM 3.3.1 (Jenkin et al., 2015).

For the practical purposes of model implementation, the bulk isomerization rates being dependent on the concentrations of $\mathrm{NO}$ and $\mathrm{HO}_{2}$, these reactions are split artificially into a unimolecular reaction and several pseudo-two-body reactions of ISOPBO 2 and ISOPDO2 with $\mathrm{NO}$ and $\mathrm{HO}_{2}$.

For the 1,5 $\mathrm{H}$-shift reactions of the $\beta$-OH-peroxy radicals, we use their theoretically estimated rates (Peeters et al., 2014) multiplied by 0.95 for ISOPBO2 and 0.94 for ISOPDO 2 to account for the small fraction of $\delta$-OH-peroxy radicals not undergoing those reactions (see Sect. 2.1.3). This parameterization of the bulk 1,5 and 1,6 $\mathrm{H}$-shifts leads to product yields in excellent agreement with an exact estimation based on the kinetic parameters of Wennberg et al. (2018), as seen in Fig. 1.

\subsubsection{Products from the isomerization of the $Z-\delta$-OH-peroxys}

The 1,6 H-shift of the Z- $\delta$-OH-peroxys $\mathrm{HOCH}_{2}-\mathrm{C}\left(\mathrm{CH}_{3}\right)=\mathrm{CH}-\mathrm{CH}_{2} \mathrm{O}_{2} \quad($ Case I) and $\mathrm{O}_{2} \mathrm{CH}_{2}-\mathrm{C}\left(\mathrm{CH}_{3}\right)=\mathrm{CH}-\mathrm{CH}_{2} \mathrm{OH}$ (Case II) forms allylic radicals, e.g. $Z-\mathrm{HOC}^{\circ} \mathrm{H}-\mathrm{C}\left(\mathrm{CH}_{3}\right)=\mathrm{CH}-\mathrm{CH}_{2} \mathrm{OOH} \Leftrightarrow$ $Z-\mathrm{HOCH}=\mathrm{C}\left(\mathrm{CH}_{3}\right)-\mathrm{C}^{\circ} \mathrm{H}-\mathrm{CH}_{2} \mathrm{OOH}$ for Case I. Therefore, two second-generation peroxys can result, peroxy $i \quad\left(Z-\mathrm{HOCH}\left(\mathrm{O}_{2}\right)-\mathrm{C}\left(\mathrm{CH}_{3}\right)=\mathrm{CH}-\mathrm{CH}_{2} \mathrm{OOH}\right)$ and peroxy ii $\left(\mathrm{Z}-\mathrm{HOCH}=\mathrm{C}\left(\mathrm{CH}_{3}\right)-\mathrm{CH}\left(\mathrm{O}_{2}\right)-\mathrm{CH}_{2} \mathrm{OOH}\right)$ in an approximate ratio of $40: 60$, and two pathways are open to product formation (Peeters et al., 2014). The subsequent chemistry is given here for Case I, unless stated otherwise. Peroxy $i$ readily eliminates $\mathrm{HO}_{2}$ at a rate of $\sim 2000 \mathrm{~s}^{-1}$ (Hermans et al., 2005) to produce $Z$ $\mathrm{O}=\mathrm{CH}-\mathrm{C}\left(\mathrm{CH}_{3}\right)=\mathrm{CH}-\mathrm{CH}_{2} \mathrm{OOH}$ (HPALD1) (Peeters et al., 2014, 2009; Crounse et al., 2011; Teng et al., 2017). Peroxy $i$ i may isomerize by a fast 1,6 enol-H-shift, promptly at $\sim 1.5 \times 10^{9} \mathrm{~s}^{-1}$ and thermally at $>10^{4} \mathrm{~s}^{-1}$, to form $\mathrm{Z}-\mathrm{O}=\mathrm{CH}-\mathrm{C}^{\circ}\left(\mathrm{CH}_{3}\right)-\mathrm{CH}(\mathrm{OOH})-\mathrm{CH}_{2} \mathrm{OOH}$ (Peeters and Nguyen, 2012; Peeters et al., 2014) that, in part, arises chemically activated, such that it can promptly undergo concerted $\mathrm{OH}$ loss and ring closure to an hydroperoxy 
Table 1. Chemical species of the oxidation mechanism of isoprene, monoterpenes, and methylbutenol (MBO).

\begin{tabular}{|c|c|}
\hline Notation & Chemical formula \\
\hline \multicolumn{2}{|c|}{$\mathrm{C}_{1}$ compounds } \\
\hline $\mathrm{HCHO}$ & $\mathrm{HCHO}$ \\
\hline $\mathrm{CO}$ & $\mathrm{CO}$ \\
\hline $\mathrm{CH}_{3} \mathrm{OH}$ & $\mathrm{CH}_{3} \mathrm{OH}$ \\
\hline $\mathrm{HCOOH}$ & $\mathrm{HC}(\mathrm{O}) \mathrm{OH}$ \\
\hline $\mathrm{CH}_{3} \mathrm{OOH}$ & $\mathrm{CH}_{3} \mathrm{OOH}$ \\
\hline $\mathrm{CH}_{3} \mathrm{OOOH}$ & $\mathrm{CH}_{3} \mathrm{OOOH}$ \\
\hline $\mathrm{CH}_{3} \mathrm{ONO}_{2}$ & $\mathrm{CH}_{3} \mathrm{ONO}_{2}$ \\
\hline НMHР & $\mathrm{HOCH}_{2} \mathrm{OOH}$ \\
\hline \multicolumn{2}{|l|}{$\mathrm{C}_{2}$ compounds } \\
\hline $\mathrm{CH}_{3} \mathrm{CHO}$ & $\mathrm{CH}_{3} \mathrm{CHO}$ \\
\hline GLYALD & $\mathrm{HOCH}_{2} \mathrm{CHO}$ \\
\hline GLY & $\mathrm{CHOCHO}$ \\
\hline $\mathrm{C}_{2} \mathrm{H}_{5} \mathrm{OH}$ & $\mathrm{C}_{2} \mathrm{H}_{5} \mathrm{OH}$ \\
\hline $\mathrm{CH}_{3} \mathrm{COOH}$ & $\mathrm{CH}_{3} \mathrm{C}(\mathrm{O}) \mathrm{OH}$ \\
\hline PAA & $\mathrm{CH}_{3} \mathrm{C}(\mathrm{O}) \mathrm{OOH}$ \\
\hline GPA & $\mathrm{OCHC}(\mathrm{O}) \mathrm{OOH}$ \\
\hline ETHLN & $\mathrm{OCHCH}_{2} \mathrm{ONO}_{2}$ \\
\hline HPAC & $\mathrm{OCHCH}_{2} \mathrm{OOH}$ \\
\hline GCO3H & $\mathrm{HOCH}_{2} \mathrm{C}(\mathrm{O}) \mathrm{OOH}$ \\
\hline GCOOH & $\mathrm{HOCH}_{2} \mathrm{C}(\mathrm{O}) \mathrm{OH}$ \\
\hline PAN & $\mathrm{CH}_{3} \mathrm{C}(\mathrm{O}) \mathrm{OONO}_{2}$ \\
\hline GPAN & $\mathrm{HOCH}_{2} \mathrm{C}(\mathrm{O}) \mathrm{OONO}_{2}$ \\
\hline VA & $\mathrm{CH}_{2}=\mathrm{CHOH}$ \\
\hline \multicolumn{2}{|l|}{$\mathrm{C}_{3}$ compounds } \\
\hline $\mathrm{CH}_{3} \mathrm{COCH}_{3}$ & $\mathrm{CH}_{3} \mathrm{C}(\mathrm{O}) \mathrm{CH}_{3}$ \\
\hline HYAC & $\mathrm{HOCH}_{2} \mathrm{C}(\mathrm{O}) \mathrm{CH}_{3}$ \\
\hline MGLY & $\mathrm{CH}_{3} \mathrm{C}(\mathrm{O}) \mathrm{CHO}$ \\
\hline $\mathrm{C}_{2} \mathrm{H}_{5} \mathrm{COOH}$ & $\mathrm{CH}_{3} \mathrm{CH}_{2} \mathrm{C}(\mathrm{O}) \mathrm{OH}$ \\
\hline NOA & $\mathrm{CH}_{3} \mathrm{C}(\mathrm{O}) \mathrm{CH}_{2} \mathrm{ONO}_{2}$ \\
\hline HPACET & $\mathrm{CH}_{3} \mathrm{C}(\mathrm{O}) \mathrm{CH}_{2} \mathrm{OOH}$ \\
\hline MVA & $\mathrm{CH}_{2}=\mathrm{C}\left(\mathrm{CH}_{3}\right) \mathrm{OH}$ \\
\hline DHA & $\mathrm{CH}_{3} \mathrm{C}(\mathrm{O}) \mathrm{CH}(\mathrm{OH})_{2}$ \\
\hline \multicolumn{2}{|l|}{$\mathrm{C}_{4}$ compounds } \\
\hline MACR & $\mathrm{CH}_{2}=\mathrm{C}\left(\mathrm{CH}_{3}\right) \mathrm{CHO}$ \\
\hline MVK & $\mathrm{CH}_{2}=\mathrm{CHC}(\mathrm{O}) \mathrm{CH}_{3}$ \\
\hline MPAN & $\mathrm{CH}_{2}=\mathrm{C}\left(\mathrm{CH}_{3}\right) \mathrm{C}(\mathrm{O}) \mathrm{OONO}_{2}$ \\
\hline $\mathrm{MCO} 3 \mathrm{H}$ & $\mathrm{CH}_{2}=\mathrm{C}\left(\mathrm{CH}_{3}\right) \mathrm{C}(\mathrm{O}) \mathrm{OOH}$ \\
\hline $\mathrm{MCOOH}$ & $\mathrm{CH}_{2}=\mathrm{C}\left(\mathrm{CH}_{3}\right) \mathrm{C}(\mathrm{O}) \mathrm{OH}$ \\
\hline MVKOOH & $0.55 \mathrm{CH}_{3} \mathrm{C}(\mathrm{O}) \mathrm{CH}(\mathrm{OOH}) \mathrm{CH}_{2} \mathrm{OH}+0.45 \mathrm{CH}_{3} \mathrm{C}(\mathrm{O}) \mathrm{CH}(\mathrm{OH}) \mathrm{CH}_{2} \mathrm{OOH}$ \\
\hline MACRNO3 & $\mathrm{OCHC}\left(\mathrm{CH}_{3}\right)\left(\mathrm{ONO}_{2}\right) \mathrm{CH}_{2} \mathrm{OH}$ \\
\hline MVKNO3 & $0.2 \mathrm{CH}_{3} \mathrm{C}(\mathrm{O}) \mathrm{CH}(\mathrm{OH}) \mathrm{CH}_{2} \mathrm{ONO}_{2}+0.8 \mathrm{CH}_{3} \mathrm{C}(\mathrm{O}) \mathrm{CH}\left(\mathrm{ONO}_{2}\right) \mathrm{CH}_{2} \mathrm{OH}$ \\
\hline MACROH & $\mathrm{HOCH}_{2} \mathrm{C}\left(\mathrm{CH}_{3}\right)(\mathrm{OH}) \mathrm{CHO}$ \\
\hline BIACETOH & $\mathrm{CH}_{3} \mathrm{C}(\mathrm{O}) \mathrm{C}(\mathrm{O}) \mathrm{CH}_{2} \mathrm{OH}$ \\
\hline DHBO & $\mathrm{CH}_{3} \mathrm{C}(\mathrm{O}) \mathrm{CH}(\mathrm{OH}) \mathrm{CH}_{2} \mathrm{OH}$ \\
\hline HOBA & $\mathrm{CH}_{3} \mathrm{C}(\mathrm{O}) \mathrm{CH}(\mathrm{OH}) \mathrm{CHO}$ \\
\hline DIHPMEK & $\mathrm{CH}_{3} \mathrm{C}(\mathrm{O}) \mathrm{CH}(\mathrm{OOH}) \mathrm{CH}_{2} \mathrm{OOH}$ \\
\hline HPKETAL & $\mathrm{CH}_{3} \mathrm{C}(\mathrm{O}) \mathrm{CH}(\mathrm{OOH}) \mathrm{CHO}$ \\
\hline HPDIAL & $\mathrm{OCHC}\left(\mathrm{CH}_{3}\right)(\mathrm{OOH}) \mathrm{CHO}$ \\
\hline HMVK & $\mathrm{CH}_{3} \mathrm{C}(\mathrm{O}) \mathrm{CH}=\mathrm{CHOH}$ \\
\hline HMAC & $\mathrm{OCHC}\left(\mathrm{CH}_{3}\right)=\mathrm{CHOH}$ \\
\hline HMML & $\mathrm { HOCH } _ { 2 } \longdiv { \mathrm { C } ( \mathrm { CH } _ { 3 } ) \mathrm { OC } } = \mathrm { O }$ \\
\hline
\end{tabular}


Table 1. Continued.

\begin{tabular}{|c|c|}
\hline Notation & Chemical formula \\
\hline \multicolumn{2}{|l|}{$\mathrm{C}_{5}$ compounds } \\
\hline ISOP & $\mathrm{CH}_{2}=\mathrm{C}\left(\mathrm{CH}_{3}\right) \mathrm{CH}=\mathrm{CH}_{2}$ \\
\hline MBO & $\mathrm{CH}_{3} \mathrm{C}(\mathrm{OH})\left(\mathrm{CH}_{3}\right) \mathrm{CH}=\mathrm{CH}_{2}$ \\
\hline HCOC5 & $\mathrm{CH}_{2}=\mathrm{C}\left(\mathrm{CH}_{3}\right) \mathrm{C}(\mathrm{O}) \mathrm{CH}_{2} \mathrm{OH}$ \\
\hline ISOPBOOH & $0.95 \mathrm{CH}_{2}=\mathrm{CHC}\left(\mathrm{CH}_{3}\right)(\mathrm{OOH}) \mathrm{CH}_{2} \mathrm{OH}+0.05 \mathrm{HOCH}_{2} \mathrm{C}\left(\mathrm{CH}_{3}\right)=\mathrm{CHCH}_{2} \mathrm{OOH}$ \\
\hline ISOPDOOH & $0.94 \mathrm{CH}_{2}=\mathrm{C}\left(\mathrm{CH}_{3}\right) \mathrm{CH}(\mathrm{OOH}) \mathrm{CH}_{2} \mathrm{OH}+0.06 \mathrm{HOCH}_{2} \mathrm{CH}=\mathrm{C}\left(\mathrm{CH}_{3}\right) \mathrm{CH}_{2} \mathrm{OOH}$ \\
\hline ISOPEOOH & $\mathrm{CH}_{2}=\mathrm{C}\left(\mathrm{CH}_{3}\right) \mathrm{CH}(\mathrm{OH}) \mathrm{CH}_{2} \mathrm{OOH}$ \\
\hline INDOOH & $\mathrm{HOCH}_{2} \mathrm{CH}\left(\mathrm{ONO}_{2}\right) \mathrm{C}\left(\mathrm{CH}_{3}\right)(\mathrm{OOH}) \mathrm{CH}_{2} \mathrm{OH}$ \\
\hline ISOPANO3 & $\mathrm{HOCH}_{2} \mathrm{C}\left(\mathrm{CH}_{3}\right)=\mathrm{CHCH}_{2} \mathrm{ONO}_{2}$ \\
\hline ISOPBNO3 & $\mathrm{CH}_{2}=\mathrm{CHC}\left(\mathrm{CH}_{3}\right)\left(\mathrm{ONO}_{2}\right) \mathrm{CH}_{2} \mathrm{OH}$ \\
\hline ISOPCNO3 & $\mathrm{HOCH}_{2} \mathrm{CH}=\mathrm{C}\left(\mathrm{CH}_{3}\right) \mathrm{CH}_{2} \mathrm{ONO}_{2}$ \\
\hline ISOPDNO3 & $\mathrm{CH}_{2}=\mathrm{C}\left(\mathrm{CH}_{3}\right) \mathrm{CH}\left(\mathrm{ONO}_{2}\right) \mathrm{CH}_{2} \mathrm{OH}$ \\
\hline ISOPENO3 & $\mathrm{CH}_{3} \mathrm{C}\left(=\mathrm{CH}_{2}\right) \mathrm{CH}(\mathrm{OH}) \mathrm{CH}_{2} \mathrm{ONO}_{2}$ \\
\hline MBONO3 & $0.67 \mathrm{CH}_{3} \mathrm{C}(\mathrm{OH})\left(\mathrm{CH}_{3}\right) \mathrm{CH}\left(\mathrm{ONO}_{2}\right) \mathrm{CH}_{2} \mathrm{OH}+0.33 \mathrm{CH}_{3} \mathrm{C}(\mathrm{OH})\left(\mathrm{CH}_{3}\right) \mathrm{CH}(\mathrm{OH}) \mathrm{CH}_{2} \mathrm{ONO}_{2}$ \\
\hline INCCO & $\mathrm{HOCH}_{2} \mathrm{C}(\mathrm{O}) \mathrm{C}\left(\mathrm{CH}_{3}\right)(\mathrm{OH}) \mathrm{CH}_{2} \mathrm{ONO}_{2}$ \\
\hline INCNO3 & $\mathrm{HOCH}_{2} \mathrm{CH}\left(\mathrm{ONO}_{2}\right) \mathrm{C}\left(\mathrm{CH}_{3}\right)(\mathrm{OH}) \mathrm{CH}_{2} \mathrm{ONO}_{2}$ \\
\hline NISOPOOHB & $0.9 \mathrm{CH}_{2}=\mathrm{CHC}\left(\mathrm{CH}_{3}\right)(\mathrm{OOH}) \mathrm{CH}_{2} \mathrm{ONO}_{2}+0.1 \mathrm{CH}_{2}=\mathrm{C}\left(\mathrm{CH}_{3}\right) \mathrm{CH}(\mathrm{OOH}) \mathrm{CH}_{2} \mathrm{ONO}_{2}$ \\
\hline NISOPOOHD & $0.84 \mathrm{HOOCH}_{2} \mathrm{CH}=\mathrm{C}\left(\mathrm{CH}_{3}\right) \mathrm{CH}_{2} \mathrm{ONO}_{2}+0.26 \mathrm{O}_{2} \mathrm{NOCH}_{2} \mathrm{CH}=\mathrm{C}\left(\mathrm{CH}_{3}\right) \mathrm{CH}_{2} \mathrm{OOH}$ \\
\hline IEPOX & $\mathrm{HOCH}_{2} \mathrm{CHOC}\left(\mathrm{CH}_{3}\right) \mathrm{CH}_{2} \mathrm{OH}$ \\
\hline ICHE & 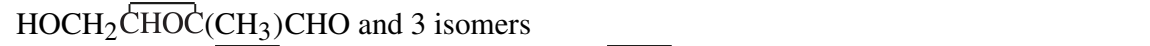 \\
\hline HPCE & $0.18 \mathrm{HOOCH}_{2} \mathrm{CHOC}\left(\mathrm{CH}_{3}\right) \mathrm{CHO}+0.82 \mathrm{OCHCHOC}\left(\mathrm{CH}_{3}\right) \mathrm{CH}_{2} \mathrm{OOH}$ \\
\hline DHHEPOX & $\mathrm{HOCH}_{2} \mathrm{C}\left(\mathrm{CH}_{3}\right)(\mathrm{OOH}) \stackrel{\mathrm{CHOCH}}{(\mathrm{OH})}$ \\
\hline $\mathrm{NC} 4 \mathrm{CHO}$ & $0.75 \mathrm{OCHCH}=\mathrm{C}\left(\mathrm{CH}_{3}\right) \mathrm{CH}_{2} \mathrm{ONO}_{2}+0.25 \mathrm{OCHC}\left(\mathrm{CH}_{3}\right)=\mathrm{CHCH}_{2} \mathrm{ONO}_{2}$ \\
\hline ISOPBOH & $\mathrm{CH}_{2}=\mathrm{CHC}\left(\mathrm{CH}_{3}\right)(\mathrm{OH}) \mathrm{CH}_{2} \mathrm{OH}$ \\
\hline ISOPDOH & $\mathrm{CH}_{2}=\mathrm{C}\left(\mathrm{CH}_{3}\right) \mathrm{CH}(\mathrm{OH}) \mathrm{CH}_{2} \mathrm{OH}$ \\
\hline HALD1 & $\mathrm{OCHC}\left(\mathrm{CH}_{3}\right)=\mathrm{CHCH}_{2} \mathrm{OH}$ \\
\hline HALD2 & $\mathrm{OCHCH}=\mathrm{C}\left(\mathrm{CH}_{3}\right) \mathrm{CH}_{2} \mathrm{OH}$ \\
\hline HPALD1 & $\mathrm{OCHC}\left(\mathrm{CH}_{3}\right)=\mathrm{CHCH}_{2} \mathrm{OOH}$ \\
\hline HPALD2 & $\mathrm{OCHCH}=\mathrm{C}\left(\mathrm{CH}_{3}\right) \mathrm{CH}_{2} \mathrm{OOH}$ \\
\hline MMAL & $\mathrm{O}=\mathrm{CCH}=\mathrm{C}\left(\mathrm{CH}_{3}\right) \mathrm{C}(=\mathrm{O}) \mathrm{O}$ \\
\hline IHNE & $0.57 \mathrm{O}_{2} \mathrm{NOCH}_{2} \mathrm{C}\left(\mathrm{CH}_{3}\right) \mathrm{OC} \mathrm{HCH}_{2} \mathrm{OH}+0.25 \mathrm{O}_{2} \mathrm{NOCH}_{2} \mathrm{C}\left(\mathrm{CH}_{3}\right)(\mathrm{OH}) \mathrm{CHOCH}_{2}$ and isomers \\
\hline \multicolumn{2}{|l|}{$\mathrm{C}_{10}$ compounds } \\
\hline APIN & $\mathrm{C}_{10} \mathrm{H}_{16}$ (sum of monoterpenes) \\
\hline APINONO2 & $\mathrm{C}_{10} \mathrm{H}_{16}(\mathrm{OH})\left(\mathrm{ONO}_{2}\right)$ \\
\hline \multicolumn{2}{|l|}{ Peroxy radicals } \\
\hline $\mathrm{CH}_{3} \mathrm{O}_{2}$ & $\mathrm{CH}_{3} \mathrm{O}_{2}$ \\
\hline $\mathrm{CH}_{3} \mathrm{CO}_{3}$ & $\mathrm{CH}_{3} \mathrm{C}(\mathrm{O}) \mathrm{O}_{2}$ \\
\hline $\mathrm{OCHCH}_{2} \mathrm{O}_{2}$ & $\mathrm{OCHCH}_{2} \mathrm{O}_{2}$ \\
\hline $\mathrm{HOCH}_{2} \mathrm{CH}_{2} \mathrm{O}_{2}$ & $\mathrm{HOCH}_{2} \mathrm{CH}_{2} \mathrm{O}_{2}$ \\
\hline $\mathrm{GCO} 3$ & $\mathrm{HOCH}_{2} \mathrm{C}(\mathrm{O}) \mathrm{O}_{2}$ \\
\hline QO2 & $\mathrm{HOCH}_{2} \mathrm{CH}_{2} \mathrm{O}_{2}$ \\
\hline ACETO2 & $\mathrm{CH}_{3} \mathrm{COCH}_{2} \mathrm{O}_{2}$ \\
\hline MVKO2 & $0.75 \mathrm{CH}_{3} \mathrm{C}(\mathrm{O}) \mathrm{CH}\left(\mathrm{O}_{2}\right) \mathrm{CH}_{2} \mathrm{OH}+0.25 \mathrm{CH}_{3} \mathrm{C}(\mathrm{O}) \mathrm{CH}(\mathrm{OH}) \mathrm{CH}_{2} \mathrm{O}_{2}$ \\
\hline MCO3 & $\mathrm{CH}_{2}=\mathrm{C}\left(\mathrm{CH}_{3}\right) \mathrm{C}(\mathrm{O}) \mathrm{O}_{2}$ \\
\hline ISOPBO2 & $0.95 \mathrm{HOCH}_{2} \mathrm{C}\left(\mathrm{CH}_{3}\right)\left(\mathrm{O}_{2}\right) \mathrm{CH}=\mathrm{CH}_{2}+0.05 \mathrm{HOCH}_{2} \mathrm{C}\left(\mathrm{CH}_{3}\right)=\mathrm{CHCH}_{2} \mathrm{O}_{2}$ \\
\hline ISOPDO2 & $0.94 \mathrm{CH}_{2}=\mathrm{C}\left(\mathrm{CH}_{3}\right) \mathrm{CH}\left(\mathrm{O}_{2}\right) \mathrm{CH}_{2} \mathrm{OH}+0.06 \mathrm{HOCH}_{2} \mathrm{CH}=\mathrm{C}\left(\mathrm{CH}_{3}\right) \mathrm{CH}_{2} \mathrm{O}_{2}$ \\
\hline ISOPEO2 & $\mathrm{CH}_{3} \mathrm{C}\left(=\mathrm{CH}_{2}\right) \mathrm{CH}(\mathrm{OH}) \mathrm{CH}_{2} \mathrm{O}_{2}$ \\
\hline DIHPCARP1 & $\mathrm{CH}_{3} \mathrm{C}(\mathrm{OO})(\mathrm{CHO}) \mathrm{CH}(\mathrm{OOH}) \mathrm{CH}_{2} \mathrm{OOH}$ \\
\hline DIHPCARP2 & $\mathrm{OCHCH}(\mathrm{OO}) \mathrm{C}\left(\mathrm{CH}_{3}\right)(\mathrm{OOH}) \mathrm{CH}_{2} \mathrm{OOH}$ \\
\hline DHPAO2 & $\mathrm{HOOCH}_{2} \mathrm{C}\left(\mathrm{CH}_{3}\right)\left(\mathrm{O}_{2}\right) \mathrm{CH}(\mathrm{OOH}) \mathrm{C}(\mathrm{O}) \mathrm{OOH}$ \\
\hline KPO2 & $0.5 \mathrm{CH}_{3} \mathrm{C}(\mathrm{O}) \mathrm{CH}\left(\mathrm{O}_{2}\right) \mathrm{CH}_{2} \mathrm{OOH}+0.5 \mathrm{CH}_{3} \mathrm{C}(\mathrm{O}) \mathrm{CH}(\mathrm{OOH}) \mathrm{CH}_{2} \mathrm{O}_{2}$ \\
\hline
\end{tabular}


Table 1. Continued.

\begin{tabular}{|c|c|}
\hline Notation & Chemical formula \\
\hline IEPOXAO2 & $\mathrm{HOCH}_{2} \mathrm{CH}(\mathrm{OH}) \mathrm{C}\left(\mathrm{CH}_{3}\right)\left(\mathrm{O}_{2}\right) \mathrm{CHO}$ \\
\hline IEPOXBO2 & $\mathrm{HOCH}_{2} \mathrm{C}(\mathrm{OH})\left(\mathrm{CH}_{3}\right) \mathrm{CH}\left(\mathrm{O}_{2}\right) \mathrm{CHO}$ \\
\hline $\mathrm{C} 59 \mathrm{O} 2$ & $\mathrm{HOCH}_{2} \mathrm{C}\left(\mathrm{CH}_{3}\right)\left(\mathrm{O}_{2}\right) \mathrm{C}(\mathrm{O}) \mathrm{CH}_{2} \mathrm{OH}$ \\
\hline INAO2 & $0.73 \mathrm{HOCH}_{2} \mathrm{C}\left(\mathrm{O}_{2}\right)\left(\mathrm{CH}_{3}\right) \mathrm{CH}(\mathrm{OH}) \mathrm{CH}_{2} \mathrm{ONO}_{2}+0.27 \mathrm{HOCH}_{2} \mathrm{C}(\mathrm{OH})\left(\mathrm{CH}_{3}\right) \mathrm{CH}\left(\mathrm{O}_{2}\right) \mathrm{CH}_{2} \mathrm{ONO}_{2}$ \\
\hline INBO2 & $0.85 \mathrm{HOCH}_{2} \mathrm{CH}\left(\mathrm{O}_{2}\right) \mathrm{C}\left(\mathrm{CH}_{3}\right)\left(\mathrm{ONO}_{2}\right) \mathrm{CH}_{2} \mathrm{OH}+0.15 \mathrm{O}_{2} \mathrm{CH}_{2} \mathrm{CH}(\mathrm{OH}) \mathrm{C}\left(\mathrm{CH}_{3}\right)\left(\mathrm{ONO}_{2}\right) \mathrm{CH}_{2} \mathrm{OH}$ \\
\hline INCO2 & $0.67 \mathrm{HOCH}_{2} \mathrm{CH}(\mathrm{OH}) \mathrm{C}\left(\mathrm{O}_{2}\right)\left(\mathrm{CH}_{3}\right) \mathrm{CH}_{2} \mathrm{ONO}_{2}+0.33 \mathrm{HOCH}{ }_{2} \mathrm{CH}\left(\mathrm{O}_{2}\right) \mathrm{C}(\mathrm{OH})\left(\mathrm{CH}_{3}\right) \mathrm{CH}_{2} \mathrm{ONO}_{2}$ \\
\hline INDO2 & $\mathrm{HOCH}_{2} \mathrm{CH}\left(\mathrm{ONO}_{2}\right) \mathrm{C}\left(\mathrm{CH}_{3}\right)\left(\mathrm{O}_{2}\right) \mathrm{CH}_{2} \mathrm{OH}$ \\
\hline NISOPO2 & $\begin{array}{l}0.45 \mathrm{O}_{2} \mathrm{CH}_{2} \mathrm{CH}=\mathrm{C}\left(\mathrm{CH}_{3}\right) \mathrm{CH}_{2} \mathrm{ONO}_{2}+0.42 \mathrm{CH}_{2}=\mathrm{CHC}\left(\mathrm{CH}_{3}\right)\left(\mathrm{O}_{2}\right) \mathrm{CH}_{2} \mathrm{ONO}_{2}+ \\
0.085 \mathrm{O}_{2} \mathrm{NOCH}_{2} \mathrm{CH}=\mathrm{C}\left(\mathrm{CH}_{3}\right) \mathrm{CH}_{2} \mathrm{O}_{2}+0.045 \mathrm{CH}_{2}=\mathrm{C}\left(\mathrm{CH}_{3}\right) \mathrm{CH}\left(\mathrm{O}_{2}\right) \mathrm{CH}_{2} \mathrm{ONO}_{2}\end{array}$ \\
\hline MBOO2 & $0.67 \mathrm{CH}_{3} \mathrm{C}(\mathrm{OH})\left(\mathrm{CH}_{3}\right) \mathrm{CH}\left(\mathrm{O}_{2}\right) \mathrm{CH}_{2} \mathrm{OH}+0.33 \mathrm{CH}_{3} \mathrm{C}(\mathrm{OH})\left(\mathrm{CH}_{3}\right) \mathrm{CH}(\mathrm{OH}) \mathrm{CH}_{2} \mathrm{O}_{2}$ \\
\hline APINOHO2 & Peroxy radical from APIN + OH \\
\hline APINO3O2 & Peroxy radical from $\mathrm{APIN}+\mathrm{O}_{3}$ \\
\hline
\end{tabular}

carbonyl epoxide, $\quad \mathrm{Z}$ - $\mathrm{HOOCH}_{2}-\overparen{\mathrm{CHOC}}\left(\mathrm{CH}_{3}\right)-\mathrm{CHO}$ (HPCE), as proposed and observed by Teng et al. (2017), and in another part lead to a third-generation peroxy, $\mathrm{Z}-\mathrm{O}=\mathrm{CH}-\mathrm{C}\left(\mathrm{CH}_{3}\right)\left(\mathrm{O}_{2}\right)-\mathrm{CH}(\mathrm{OOH})-\mathrm{CH}_{2} \mathrm{OOH}$ (DIHPCARP1) (Peeters et al., 2014). The DIHPCARP radicals have been suggested (Peeters et al., 2014) to either undergo a fast aldehyde- $\mathrm{H}$-shift to eliminate $\mathrm{CO}$ and expel $\mathrm{OH}$ and form dihydroperoxy carbonyls, or react with $\mathrm{NO}$ and $\mathrm{HO}_{2}$, to result mainly in $\mathrm{OH}+\mathrm{CH}_{3} \mathrm{C}(\mathrm{O}) \mathrm{CHO}(\mathrm{MGLY})+\mathrm{HOOCH}_{2} \mathrm{CHO}$ (HPAC) (Case I) or $\mathrm{OH}+\mathrm{OCHCHO}+\mathrm{CH}_{3} \mathrm{C}(\mathrm{O}) \mathrm{CH}_{2} \mathrm{OOH}$ (HPACET) (Case II). While the $\mathrm{CO}$ elimination above may be fast enough to outrun $\mathrm{O}_{2}$ addition for Case I (Novelli et al., 2018b), this appears less likely for Case II, for which the barrier should be about $2 \mathrm{kcal} \mathrm{mol}^{-1}$ higher (Méreau et al., 2001). Note that HPAC and HPACET were observed by Teng et al. (2017) but in a ratio to HPALDs nearly independent of the NO level. Secondly, it is estimated using statistical rate theory that the 1,6 enol-H-shift above can occur at about $50 \%$ while its peroxy precursor is still chemically activated, such that the resulting radical contains close to $30 \mathrm{kcal} \mathrm{mol}^{-1}$ internal energy (Peeters et al., 2014), sufficient for prompt HPCE epoxide formation.

In this work, the quantitative product distribution from the $1,6 \mathrm{H}$-shift of the $Z-\delta$-OH-peroxys is adopted from the recent experimental study of Berndt et al. (2019), supported and complemented by computational results of the LIM1 paper, i.e. Peeters et al. (2014). Note that the 1,6 H-shifts of the $Z$ - $\delta$-OH-peroxys occur for $\sim 85 \%$ by tunnelling (Coote et al., 2003) at energies lower than $2 \mathrm{kcal} \mathrm{mol}^{-1}$ below the barrier top, such that the Boltzmann population there is only marginally affected by the $\mathrm{O}_{2}$ loss that occurs only at energies above this range; therefore, there is no reason to suspect (Wennberg et al., 2018) that the agreement between experimental results (Teng et al., 2017) and the rate constants predicted using Transition State Theory by Peeters et al. (2014) is fortuitous. The Berndt et al. (2019) investigation offers several advantages: (i) the reaction time was so short ( $8 \mathrm{~s})$ that no secondary products could be formed; (ii) due to the absence of $\mathrm{NO}$ and near absence of $\mathrm{HO}_{2}$, essentially only the products of the $Z-\delta$-OH-peroxy $1,6 \mathrm{H}$-shift could be formed, excluding potential interferences; (iii) the peroxy radicals could also be observed; and (iv) the sampled products and peroxy radicals could be quasi-quantitatively converted into ion complexes, which were detected by highresolution mass spectrometry capable of measuring concentrations as low as $10^{4} \mathrm{~cm}^{-3}$. Hydroxyl radicals were prepared by reacting $10^{12} \mathrm{~cm}^{-3}$ of $\mathrm{O}_{3}$ with $2 \times 10^{11} \mathrm{~cm}^{-3}$ of tetramethylethylene, in the presence of $2.5 \times 10^{12} \mathrm{~cm}^{-3}$ of isoprene. At $8 \mathrm{~s}$ reaction time, the modelled total ISOPOO concentration is $1.2 \times 10^{9} \mathrm{~cm}^{-3}$, of which $6 \times 10^{6} \mathrm{~cm}^{-3}$ were $Z-\delta-O H-$ peroxys $\left(50 \%\right.$ Case I isomer $\mathrm{HOCH}_{2} \mathrm{C}\left(\mathrm{CH}_{3}\right)=\mathrm{CHCH}_{2} \mathrm{O}_{2}$ and $50 \%$ Case II isomer $\mathrm{O}_{2} \mathrm{CH}_{2} \mathrm{C}\left(\mathrm{CH}_{3}\right)=\mathrm{CHCH}_{2} \mathrm{OH}$ at $\left.8 \mathrm{~s}\right)$. Integrated over the entire reaction time of $8 \mathrm{~s}$, the modelled ratio of these two peroxys is circa $0.8: 1.0$. Using the isomer-specific $1,6 \mathrm{H}$-shift rates of 0.36 and $3.7 \mathrm{~s}^{-1}$ for $Z$ $\delta$-OH-peroxys I and II (Teng et al., 2017), the expected total formation rate of isomerization products at $8 \mathrm{~s}$ is $1.2 \times$ $10^{6} \mathrm{~cm}^{-3} \mathrm{~s}^{-1}$. For these conditions, Berndt et al. (2019) measured the following concentrations at $8 \mathrm{~s}: \mathrm{C}_{5} \mathrm{H}_{8} \mathrm{O}_{3}$ (HPALDs) at $2.3 \times 10^{7} \mathrm{~cm}^{-3}, \mathrm{C}_{5} \mathrm{H}_{8} \mathrm{O}_{4}$ (hydroperoxy carbonyl epoxides) at $4.6 \times 10^{6} \mathrm{~cm}^{-3}, \mathrm{C}_{4} \mathrm{H}_{8} \mathrm{O}_{5}$ (dihydroperoxy carbonyls) at $6.2 \times 10^{5} \mathrm{~cm}^{-3}, \mathrm{C}_{5} \mathrm{H}_{9} \mathrm{O}_{5}$ (the second-generation peroxys above) at $1.7 \times 10^{6} \mathrm{~cm}^{-3}$, and $\mathrm{C}_{5} \mathrm{H}_{9} \mathrm{O}_{7}$ (the third-generation peroxys) at $3.5 \times 10^{5} \mathrm{~cm}^{-3}$. In principle, these values are minimum concentrations. Neither HPAC nor HPACET were detected. The detected product and peroxy concentrations together account for $60 \%$ of the modelled total products at $8 \mathrm{~s}$ using the experimental kinetic parameters of Teng et al. (2017), which, together with the uncertainties, leaves room for some other products. The theoretically derived parameters of Peeters et al. (2014) predict a higher product formation from the $Z-\delta-O H$-peroxy isomerization at $8 \mathrm{~s}$, but this is due to a too low LIM1-predicted $\mathrm{O}_{2}$ loss from the peroxys, such that the populations of the $Z-\delta-\mathrm{OH}$-peroxys at $8 \mathrm{~s}$ are still too close to their high initial formation fraction and attain their much lower final steady-state fraction too late. 
Table 2. Chemical mechanism and rates. Units for first-, second-, and third-order reactions are $\mathrm{s}^{-1}, \mathrm{~cm}^{3} \mathrm{molec} . \mathrm{s}^{-1}$, and $\mathrm{cm}^{6}$ molec. ${ }^{-2} \mathrm{~s}^{-1}$, respectively. Read $2.7(-11)$ as $2.7 \times 10^{-11} . T$ is temperature $(\mathrm{K}) ;[M]$ is air density (molec. $\mathrm{cm}^{-3}$ ); $K_{\mathrm{RO} 2 \mathrm{NO}}=2.7(-12) \exp (350 / T)$; and the PAN-like compounds formation and decomposition rates are calculated with $k=$ $\frac{k_{0}[M]}{+k_{0}[M] / k_{\infty}} 0.3^{\left\{1+\left[\log _{10}\left(k_{0}[M] / k_{\infty}\right) / 1.414\right]^{2}\right\}^{-1}}$. References: 1, MCM (Saunders et al., 2003; Jenkin et al., 2015); 2, Nguyen et al. (2016); 3, Wennberg et al. (2018); 4, Liu et al. (2013); 5, Peeters and Müller (2010); 6, Capouet et al. (2004); 7, Atkinson et al. (2006); 8, Peeters et al. (2014); 9, St. Clair et al. (2016); 10, D’Ambro et al. (2017); 11, Lee et al. (2014); 12, Jacobs et al. (2014); 13, Paulot et al. (2009b); 14, Bates et al. (2016); 15, Schwantes et al. (2015); 16, Xiong et al. (2016); 17, Crounse et al. (2012); 18, Gross et al. (2014); 19, Burkholder et al. (2015); 20, Nguyen et al. (2015a); 21, Galloway et al. (2011); 22, Praske et al. (2015); 23, Vu et al. (2013); 24, Baeza-Romero et al. (2007); 25, Magneron et al. (2005); 26, Taraborrelli et al. (2012); 28, So et al. (2014); 29, Assaf et al. (2016); 30, Assaf et al. (2018); 31, Müller et al. (2016); 32, Allen et al. (2018); 34, Chan et al. (2009). The notes are provided in Sect. 2.8.

\begin{tabular}{|c|c|c|c|}
\hline Reaction & Rate & Ref. & Note \\
\hline \multicolumn{4}{|l|}{$\mathrm{C}_{5}$ compounds } \\
\hline $\begin{array}{l}\text { ISOP }+ \text { OH } \rightarrow 0.586 \text { ISOPBO } 2+0.344 \text { ISOPDO } 2+0.02 \text { ISOPEO } 2 \\
\quad+0.10 \mathrm{HO}_{2}+0.05 \mathrm{ACETO} 2+0.05 \mathrm{HCHO}+0.05 \mathrm{CO}_{2}\end{array}$ & $2.7(-11) \exp (360 / T)$ & & N1 \\
\hline $\mathrm{ISOP}+\mathrm{NO}_{3} \rightarrow \mathrm{NISOPO} 2$ & $3.15(-12) \exp (-450 / T)$ & 1 & \\
\hline $\begin{array}{l}\text { ISOP }+\mathrm{O}_{3} \rightarrow 0.41 \mathrm{MACR}+0.17 \mathrm{MVK}+0.86 \mathrm{HCHO}+0.03 \mathrm{MCOOH} \\
\quad+0.3 \mathrm{CO}_{2}+0.3 \mathrm{HO}_{2}+0.1 \mathrm{CH}_{3} \mathrm{O}_{2}+0.24 \mathrm{CO}+0.05 \mathrm{CH}_{3} \mathrm{CO}_{3} \\
\quad+0.14 \mathrm{OH}+0.58\left(0.55 \mathrm{HMHP}+0.4 \mathrm{HCHO}+0.4 \mathrm{H}_{2} \mathrm{O}_{2}\right. \\
\quad+0.05 \mathrm{HCOOH})\end{array}$ & $1.03(-14) \exp (-1995 / T)$ & 2 & $\mathrm{~N} 2$ \\
\hline $\begin{array}{l}\mathrm{ISOPBO} 2+\mathrm{NO} \rightarrow \mathrm{NO}_{2}+0.95 \mathrm{MVK}+0.95 \mathrm{HCHO}+0.973 \mathrm{HO}_{2} \\
\quad+0.023 \mathrm{HALD} 1+0.027 \mathrm{MVKOOH}+0.027 \mathrm{CO}+0.027 \mathrm{OH}\end{array}$ & $K_{\mathrm{RO} 2 \mathrm{NO}} \cdot Y_{\mathrm{oxy}}(T, M, 6,1.19)$ & 3 & N3 \\
\hline ISOPBO $2+\mathrm{NO} \rightarrow 0.96 \mathrm{ISOPBNO} 3+0.04 \mathrm{ISOPANO} 3$ & $K_{\mathrm{RO} 2 \mathrm{NO}} \cdot Y_{\mathrm{nit}}(T, M, 6,1.19)$ & 3 & N3 \\
\hline $\begin{array}{l}\mathrm{ISOPBO}_{2}+\mathrm{NO}_{3} \rightarrow \mathrm{NO}_{2}+0.95 \mathrm{MVK}+0.95 \mathrm{HCHO}+0.973 \mathrm{HO}_{2} \\
\quad+0.023 \mathrm{HALD} 1+0.027 \mathrm{MVKOOH}+0.027 \mathrm{CO}+0.027 \mathrm{OH}\end{array}$ & $2.3(-12)$ & 1,3 & \\
\hline $\begin{array}{l}\mathrm{ISOPBO} 2+\mathrm{HO}_{2} \rightarrow 0.94 \mathrm{ISOPBOOH}+0.06 \mathrm{OH} \\
\quad+0.06 \mathrm{MVK}+0.06 \mathrm{HCHO}+0.06 \mathrm{HO}_{2}\end{array}$ & $2.1(-13) \exp (1300 / T)$ & $1,3,4$ & \\
\hline $\mathrm{ISOPBO} 2+\mathrm{ISOPBO} 2 \rightarrow 2 \mathrm{MVK}+2 \mathrm{HCHO}+2 \mathrm{HO}_{2}$ & $6.6(-14)$ & 3 & \\
\hline $\begin{array}{l}\text { ISOPBO } 2+\text { ISOPBO } 2 \rightarrow 0.5 \mathrm{HO}_{2}+0.5 \mathrm{HALD} 1+0.5 \mathrm{CO}+0.5 \mathrm{OH} \\
\quad+0.5 \mathrm{MVKOOH}\end{array}$ & $1.1(-13)$ & 3 & \\
\hline $\begin{array}{l}\text { ISOPBO } 2+\text { ISOPDO } 2 \rightarrow 0.9 \mathrm{MVK}+1.8 \mathrm{HCHO}+1.8 \mathrm{HO}_{2} \\
\quad+0.1 \mathrm{ISOPBOH}+0.9 \mathrm{MACR}+0.1 \mathrm{HCOC} 5\end{array}$ & $3.08(-12)$ & 3 & \\
\hline $\begin{array}{l}\mathrm{ISOPBO} 2+\mathrm{CH}_{3} \mathrm{O}_{2} \rightarrow 0.5 \mathrm{MVK}+1.5 \mathrm{HCHO}+0.7 \mathrm{HO}_{2} \\
\quad+0.5 \mathrm{ISOPBOH}\end{array}$ & $2.0(-12)$ & 3 & \\
\hline $\mathrm{ISOPBO} 2+\mathrm{CH}_{3} \mathrm{CO}_{3} \rightarrow \mathrm{MVK}+\mathrm{HCHO}+\mathrm{HO}_{2}+\mathrm{CH}_{3} \mathrm{O}_{2}+\mathrm{CO}_{2}$ & $1.8(-12) \exp (500 / T)$ & 6,7 & \\
\hline $\begin{array}{l}\text { ISOPBO } 2 \rightarrow 0.75 \mathrm{HPALD} 1+0.75 \mathrm{HO}_{2}+0.15 \mathrm{HPCE} \\
\quad+0.25 \mathrm{OH}+0.1 \mathrm{CO}+0.1 \mathrm{DIHPMEK}\end{array}$ & $\begin{array}{l}3.409(+12) \exp (-10698 / T) \\
+2.89(-15) \exp (414 / T) \cdot[\mathrm{NO}] \\
+2.26(-16) \exp (1364 / T) \cdot\left[\mathrm{HO}_{2}\right]\end{array}$ & & $\mathrm{N} 4$ \\
\hline $\mathrm{ISOPBO} 2 \rightarrow \mathrm{MVK}+\mathrm{HCHO}+\mathrm{OH}$ & $9.9(+10) \exp (-9746 / T)$ & 8 & \\
\hline $\mathrm{ISOPBOOH}+\mathrm{OH} \rightarrow 0.85 \mathrm{IEPOX}+0.15 \mathrm{DHHEPOX}+\mathrm{OH}$ & $1.7(-11) \exp (390 / T)$ & $9,3,10$ & N6 \\
\hline $\begin{array}{l}\mathrm{ISOPBOOH}+\mathrm{OH} \rightarrow 0.75 \mathrm{ISOPBO} 2+0.2 \mathrm{HCOOH}+0.3 \mathrm{HO}_{2} \\
\quad+0.05 \mathrm{HCHO}+0.05 \mathrm{OH}+0.25 \mathrm{MVK}\end{array}$ & $4.6(-12) \exp (200 / T)$ & 9,3 & N7 \\
\hline $\begin{array}{l}\mathrm{ISOPDO} 2+\mathrm{NO} \rightarrow \mathrm{NO}_{2}+0.94 \mathrm{MACR}+0.94 \mathrm{HCHO}+\mathrm{HO}_{2} \\
\quad+0.027 \mathrm{HALD} 2+0.033 \mathrm{HYAC}+0.066 \mathrm{CO}+0.066 \mathrm{OH}\end{array}$ & $K_{\mathrm{RO} 2 \mathrm{NO}} \cdot Y_{\mathrm{oxy}}(T, M, 6,1.3)$ & 1,3 & N3 \\
\hline ISOPDO $2+\mathrm{NO} \rightarrow 0.944 \mathrm{ISOPDNO} 3+0.056 \mathrm{ISOPCNO} 3$ & $K_{\mathrm{RO} 2 \mathrm{NO}} \cdot Y_{\mathrm{nit}}(T, M, 6,1.3)$ & 1,3 & N3 \\
\hline $\begin{array}{l}\mathrm{ISOPDO} 2+\mathrm{NO}_{3} \rightarrow \mathrm{NO}_{2}+0.94 \mathrm{MACR}+0.94 \mathrm{HCHO}+\mathrm{HO}_{2} \\
\quad+0.027 \mathrm{HALD} 2+0.037 \mathrm{HYAC}+0.066 \mathrm{CO}+0.066 \mathrm{OH}\end{array}$ & $2.3(-12)$ & 1 & \\
\hline $\begin{array}{l}\text { ISOPDO } 2+\mathrm{HO}_{2} \rightarrow 0.941 \mathrm{ISOPDOOH}+0.059 \mathrm{OH} \\
\quad+0.059 \mathrm{MACR}+0.059 \mathrm{HCHO}+0.059 \mathrm{HO}_{2}\end{array}$ & $2.1(-13) \exp (1300 / T)$ & 1,3 & \\
\hline $\begin{array}{l}\mathrm{ISOPDO} 2+\mathrm{ISOPDO} 2 \rightarrow 1.6 \mathrm{MACR}+1.6 \mathrm{HCHO}+1.6 \mathrm{HO}_{2} \\
\quad+0.2 \mathrm{HCOC} 5+0.2 \mathrm{ISOPDOH}\end{array}$ & $5.74(-12)$ & 3 & \\
\hline $\begin{array}{l}\text { ISOPDO } 2+\mathrm{CH}_{3} \mathrm{O}_{2} \rightarrow 0.5 \mathrm{MACR}+1.25 \mathrm{HCHO}+\mathrm{HO}_{2} \\
\quad+0.25 \mathrm{ISOPDOH}+0.25 \mathrm{HCOC} 5+0.25 \mathrm{CH}_{3} \mathrm{OH}\end{array}$ & $2.0(-12)$ & 3 & \\
\hline $\begin{array}{l}\text { ISOPDO } 2+\mathrm{CH}_{3} \mathrm{CO}_{3} \rightarrow 0.9 \mathrm{MACR}+0.9 \mathrm{HCHO}+0.9 \mathrm{HO}_{2} \\
\quad+0.9 \mathrm{CH}_{3} \mathrm{O}_{2}+0.9 \mathrm{CO}_{2}+0.1 \mathrm{CH}_{3} \mathrm{COOH}+0.1 \mathrm{HCOC} 5\end{array}$ & $2.0(-12) \exp (500 / T)$ & 6,7 & \\
\hline $\begin{array}{l}\text { ISOPDO } 2 \rightarrow 0.75 \mathrm{HPALD} 2+0.75 \mathrm{HO}_{2}+0.15 \mathrm{HPCE} \\
\quad+0.15 \mathrm{OH}+0.1 \mathrm{DHPAO} 2\end{array}$ & $\begin{array}{l}4.253(+8) \exp (-7254 / T) \\
+6.29(-19) \exp (4012 / T) \cdot[\mathrm{NO}] \\
+4.9(-20) \exp (4962 / T) \cdot\left[\mathrm{HO}_{2}\right]\end{array}$ & & N4 \\
\hline $\mathrm{ISOPDO} 2 \rightarrow \mathrm{MACR}+\mathrm{HCHO}+\mathrm{OH}$ & $1.77(+11) \exp (-9752 / T)$ & 8 & \\
\hline $\mathrm{HPCE}+\mathrm{OH} \rightarrow 1.82 \mathrm{CO}+0.82 \mathrm{OH}+0.82 \mathrm{HPACET}+0.18 \mathrm{KPO} 2$ & $2.5(-11)$ & & N5 \\
\hline
\end{tabular}


Table 2. Continued.

\begin{tabular}{|c|c|c|c|}
\hline Reaction & Rate & Ref. & Note \\
\hline $\begin{array}{l}\mathrm{KPO} 2+\mathrm{NO} \rightarrow \mathrm{NO}_{2}+0.5 \mathrm{CH}_{3} \mathrm{CO}_{3}+0.5 \mathrm{HPAC} \\
\quad+0.5 \mathrm{HCHO}+0.5 \mathrm{OH}+0.5 \mathrm{MGLY}\end{array}$ & $2.7(-12) \exp (350 / T)$ & & N5 \\
\hline $\begin{array}{l}\mathrm{KPO} 2+\mathrm{NO}_{3} \rightarrow \mathrm{NO}_{2}+0.5 \mathrm{CH}_{3} \mathrm{CO}_{3}+0.5 \mathrm{HPAC} \\
\quad+0.5 \mathrm{HCHO}+0.5 \mathrm{OH}+0.5 \mathrm{MGLY}\end{array}$ & $2.3(-12)$ & & N5 \\
\hline $\begin{array}{l}\mathrm{KPO} 2+\mathrm{HO}_{2} \rightarrow \mathrm{OH}+0.5 \mathrm{CH}_{3} \mathrm{CO}_{3}+0.5 \mathrm{HPAC} \\
\quad+0.5 \mathrm{HCHO}+0.5 \mathrm{OH}+0.5 \mathrm{MGLY}\end{array}$ & $2.26(-13) \exp (1300 / T)$ & & N5 \\
\hline $\mathrm{DHPAO} 2+\mathrm{NO} \rightarrow \mathrm{NO}_{2}+\mathrm{HPACET}+\mathrm{OH}+\mathrm{PGA}$ & $2.7(-12) \exp (350 / T)$ & & N5 \\
\hline $\mathrm{DHPAO} 2+\mathrm{NO}_{3} \rightarrow \mathrm{NO}_{2}+\mathrm{HPACET}+\mathrm{OH}+\mathrm{PGA}$ & $2.3(-12)$ & & \\
\hline $\mathrm{DHPAO} 2+\mathrm{HO}_{2} \rightarrow \mathrm{OH}+\mathrm{HPACET}+\mathrm{OH}+\mathrm{PGA}$ & $2.64(-13) \exp (1300 / T)$ & & N5 \\
\hline $\mathrm{ISOPDOOH}+\mathrm{OH} \rightarrow 0.85 \mathrm{IEPOX}+0.15 \mathrm{DHHEPOX}+\mathrm{OH}$ & $3.0(-11) \exp (390 / T)$ & $9,3,10$ & N6 \\
\hline $\begin{array}{l}\mathrm{ISOPDOOH}+\mathrm{OH} \rightarrow 0.6 \mathrm{ISOPDO} 2+0.32 \mathrm{HCOOH}+0.48 \mathrm{HO}_{2} \\
\quad+0.08 \mathrm{HCHO}+0.08 \mathrm{OH}+0.4 \mathrm{MACR}\end{array}$ & $4.1(-12) \exp (200 / T)$ & 9,3 & N8 \\
\hline $\mathrm{ISOPEO} 2+\mathrm{NO} \rightarrow \mathrm{MACR}+\mathrm{HO}_{2}+\mathrm{HCHO}+\mathrm{NO}_{2}$ & $K_{\mathrm{RO} 2 \mathrm{NO}} \cdot Y_{\mathrm{oxy}}(T, M, 6,1.27)$ & 1,3 & N3 \\
\hline ISOPEO $2+\mathrm{NO} \rightarrow$ ISOPENO3 & $K_{\mathrm{RO} 2 \mathrm{NO}} \cdot Y_{\mathrm{nit}}(T, M, 6,1.27)$ & 1,3 & N3 \\
\hline $\mathrm{ISOPEO} 2+\mathrm{HO}_{2} \rightarrow \mathrm{ISOPEOOH}$ & $2.1(-13) \exp (1300 / T)$ & 1,3 & \\
\hline $\begin{array}{l}\text { ISOPEO } 2+\text { ISOPBO } 2 \rightarrow 0.7 \mathrm{MVK}+1.4 \mathrm{HCHO}+1.4 \mathrm{HO}_{2} \\
\quad+0.3 \mathrm{ISOPBOH}+0.7 \mathrm{MACR}+0.3 \mathrm{HCOC} 5\end{array}$ & $1.2(-12)$ & 5 & \\
\hline $\begin{array}{l}\mathrm{ISOPEO} 2+\mathrm{ISOPDO} 2 \rightarrow \mathrm{MACR}+\mathrm{HCHO}+\mathrm{HO}_{2}+0.5 \mathrm{HCOC} 5 \\
\quad+0.5 \mathrm{ISOPDOH}\end{array}$ & $1.1(-11)$ & 5 & \\
\hline $\begin{array}{l}\mathrm{ISOPEO} 2+\mathrm{ISOPEO} 2 \rightarrow \mathrm{MACR}+\mathrm{HCHO}+\mathrm{HO}_{2} \\
\quad+0.5 \mathrm{HCOC} 5+0.5 \mathrm{ISOPDOH}\end{array}$ & $5.0(-12)$ & 5 & \\
\hline $\mathrm{ISOPEOOH}+\mathrm{OH} \rightarrow 0.83 \mathrm{HYAC}+0.83 \mathrm{GLY}+0.17 \mathrm{MACR}+\mathrm{HO}_{2}$ & $1.0(-10)$ & 1 & N9 \\
\hline $\mathrm{ISOPENO} 3+\mathrm{OH} \rightarrow \mathrm{HYAC}+\mathrm{ETHLN}+\mathrm{HO}_{2}$ & $6.0(-11)$ & 1,11 & N9 \\
\hline $\mathrm{ISOPBNO} 3+\mathrm{OH} \rightarrow 0.85 \mathrm{INBO} 2+0.15 \mathrm{IEPOX}+0.15 \mathrm{NO}_{2}$ & $8.4(-12) \exp (390 / T)$ & 1,3 & \\
\hline $\mathrm{INBO} 2 \rightarrow 2 \mathrm{HO}_{2}+\mathrm{CO}+\mathrm{MVKOOH}+\mathrm{NO}_{2}$ & $7.5(12) \exp (-10000 / T)$ & 3 & N11 \\
\hline $\mathrm{INBO} 2+\mathrm{NO} \rightarrow \mathrm{HNO}_{3}$ & $K_{\mathrm{RO} 2 \mathrm{NO}} \cdot Y_{\mathrm{nit}}(T, M, 11,6.3)$ & 1,3 & N12 \\
\hline $\begin{array}{l}\mathrm{INBO} 2+\mathrm{NO} \rightarrow 1.85 \mathrm{NO}_{2}+0.85 \mathrm{GLYALD}+0.85 \mathrm{HYAC} \\
\quad+0.15 \mathrm{MACRNO} 3+0.15 \mathrm{HO}_{2}+0.15 \mathrm{HCHO}\end{array}$ & $K_{\mathrm{RO} 2 \mathrm{NO}} \cdot Y_{\mathrm{oxy}}(T, M, 11,6.3)$ & $1,13,3$ & \\
\hline $\begin{array}{l}\mathrm{INBO} 2+\mathrm{NO}_{3} \rightarrow 1.85 \mathrm{NO}_{2}+0.85 \mathrm{GLYALD}+0.85 \mathrm{HYAC} \\
\quad+0.15 \mathrm{MACRNO} 3+0.85 \mathrm{HO}_{2}+0.15 \mathrm{HCHO}\end{array}$ & $2.3(-12)$ & 1 & \\
\hline $\mathrm{INBO} 2+\mathrm{HO}_{2} \rightarrow \mathrm{HNO}_{3}$ & $2.5(-13) \exp (1300 / T)$ & 1,3 & N13 \\
\hline $\mathrm{ISOPDNO} 3+\mathrm{OH} \rightarrow 0.85 \mathrm{INDO} 2+0.15 \mathrm{IEPOX}+0.15 \mathrm{NO}_{2}$ & $3.9(-11)$ & 1,3 & \\
\hline $\mathrm{INDO} 2 \rightarrow 3 \mathrm{HO}_{2}+2 \mathrm{CO}+\mathrm{OH}+\mathrm{HYAC}+\mathrm{NO}_{2}$ & $7.5(12) \exp (-10000 / T)$ & 3 & N14 \\
\hline $\mathrm{INDO} 2+\mathrm{NO} \rightarrow \mathrm{HNO}_{3}$ & $K_{\mathrm{RO} 2 \mathrm{NO}} \cdot Y_{\mathrm{nit}}(T, M, 11,7.9)$ & 1,3 & N12 \\
\hline $\mathrm{INDO} 2+\mathrm{NO} \rightarrow \mathrm{HCHO}+\mathrm{HO}_{2}+\mathrm{MVKNO} 3+\mathrm{NO}_{2}$ & $K_{\mathrm{RO} 2 \mathrm{NO}} \cdot Y_{\mathrm{Oxy}}(T, M, 11,7.9)$ & $1,3,11,12$ & \\
\hline $\mathrm{INDO} 2+\mathrm{NO}_{3} \rightarrow \mathrm{HCHO}+\mathrm{HO}_{2}+\mathrm{MVKNO} 3+\mathrm{NO}_{2}$ & $2.3(-12)$ & 1 & \\
\hline $\begin{array}{l}\mathrm{INDO} 2+\mathrm{HO}_{2} \rightarrow 0.39 \mathrm{INDOOH}+0.65 \mathrm{HCHO}+0.65 \mathrm{HO}_{2} \\
\quad+0.65 \mathrm{MVKNO} 3\end{array}$ & $2.5(-13) \exp (1300 / T)$ & 1,3 & \\
\hline $\begin{array}{l}\mathrm{INDOOH}+\mathrm{OH} \rightarrow 0.39 \mathrm{INDO} 2+1.22 \mathrm{HO}_{2}+0.61 \mathrm{CO} \\
\quad+0.61 \mathrm{MVKNO} 3+0.61 \mathrm{OH}\end{array}$ & $9.2(-12)$ & 1 & N15 \\
\hline $\mathrm{IEPOX}+\mathrm{OH} \rightarrow 0.19 \mathrm{ICHE}+0.58 \mathrm{IEPOXAO} 2+0.23 \mathrm{IEPOXBO} 2$ & $4.4(-11) \exp (-400 / T)$ & 3 & N16 \\
\hline $\mathrm{ICHE}+\mathrm{OH} \rightarrow 0.28 \mathrm{OH}+1.28 \mathrm{CO}+0.28 \mathrm{HYAC}+0.72 \mathrm{MVKO} 2$ & $1.5(-11)$ & & N17 \\
\hline $\mathrm{ICHE}+\mathrm{OH} \rightarrow \mathrm{CO}+\mathrm{HO}_{2}+0.28 \mathrm{HPDIAL}+0.72 \mathrm{HPKETAL}$ & $2.2(-11) \exp (-400 / T)$ & & N18 \\
\hline $\mathrm{IEPOXAO} 2 \rightarrow \mathrm{DHBO}+\mathrm{OH}+\mathrm{CO}$ & $1.0(7) \exp (-5000 / T)$ & 3 & N19 \\
\hline $\begin{array}{l}\mathrm{IEPOXAO} 2 \rightarrow \mathrm{CO}+2.5 \mathrm{HO}_{2}+1.5 \mathrm{OH}+0.5 \mathrm{HOBA} \\
\quad+0.5 \mathrm{HPDIAL}\end{array}$ & $1.875(13) \exp (-10000 / T)$ & 3 & $\mathrm{~N} 20$ \\
\hline $\begin{array}{l}\mathrm{IEPOXAO} 2+\mathrm{NO} \rightarrow \mathrm{NO}_{2}+\mathrm{HO}_{2}+0.8 \mathrm{MGLY}+0.8 \mathrm{GLYALD} \\
\quad+0.2 \mathrm{DHBO}+0.2 \mathrm{CO}\end{array}$ & $K_{\mathrm{RO} 2 \mathrm{NO}}$ & 1,3 & \\
\hline $\begin{array}{l}\mathrm{IEPOXAO} 2+\mathrm{HO}_{2} \rightarrow \mathrm{OH}+\mathrm{HO}_{2}+0.8 \mathrm{MGLY}+0.8 \mathrm{GLYALD} \\
\quad+0.2 \mathrm{DHBO}+0.2 \mathrm{CO}\end{array}$ & $1.6(-13) \exp (1300 / T)$ & 3 & $\mathrm{~N} 21$ \\
\hline $\mathrm{IEPOXAO} 2+\mathrm{HO}_{2} \rightarrow \mathrm{CO}+\mathrm{HO}_{2}+\mathrm{OH}+\mathrm{DHBO}$ & $0.8(-13) \exp (1300 / T)$ & 3 & $\mathrm{~N} 22$ \\
\hline $\mathrm{IEPOXBO} 2 \rightarrow \mathrm{MACROH}+\mathrm{OH}+\mathrm{CO}$ & $1.0(7) \exp (-5000 / T)$ & 3 & N19 \\
\hline $\mathrm{IEPOXBO} 2 \rightarrow 1.5 \mathrm{CO}+3 \mathrm{HO}_{2}+0.5 \mathrm{MGLY}+0.5 \mathrm{HPKETAL}$ & $1.875(13) \exp (-10000 / T)$ & 3 & $\mathrm{~N} 23$ \\
\hline $\begin{array}{l}\mathrm{IEPOXBO} 2+\mathrm{NO} \rightarrow \mathrm{NO}_{2}+\mathrm{HO}_{2}+0.8 \mathrm{GLY}+0.8 \mathrm{HYAC} \\
\quad+0.2 \mathrm{MACROH}+0.2 \mathrm{CO}\end{array}$ & $K_{\mathrm{RO} 2 \mathrm{NO}}$ & 1,3 & \\
\hline $\begin{array}{l}\mathrm{IEPOXBO} 2+\mathrm{HO}_{2} \rightarrow \mathrm{OH}+\mathrm{HO}_{2}+0.8 \mathrm{GLY}+0.8 \mathrm{HYAC} \\
\quad+0.2 \mathrm{MACROH}+0.2 \mathrm{CO}\end{array}$ & $1.6(-13) \exp (1300 / T)$ & 3 & $\mathrm{~N} 21$ \\
\hline
\end{tabular}


Table 2. Continued.

\begin{tabular}{|c|c|c|c|}
\hline Reaction & Rate & Ref. & Note \\
\hline $\mathrm{IEPOXBO} 2+\mathrm{HO}_{2} \rightarrow \mathrm{CO}+\mathrm{HO}_{2}+\mathrm{OH}+\mathrm{MACROH}$ & $0.8(-13) \exp (1300 / T)$ & 3 & $\mathrm{~N} 24$ \\
\hline $\mathrm{HCOC} 5+\mathrm{OH} \rightarrow \mathrm{C} 59 \mathrm{O} 2$ & $3.81(-11)$ & 1 & \\
\hline $\mathrm{C} 59 \mathrm{O} 2+\mathrm{NO} \rightarrow \mathrm{HYAC}+\mathrm{GCO} 3+\mathrm{NO}_{2}$ & $K_{\mathrm{RO} 2 \mathrm{NO}}$ & 1 & \\
\hline $\mathrm{C} 59 \mathrm{O} 2+\mathrm{NO}_{3} \rightarrow \mathrm{HYAC}+\mathrm{GCO} 3+\mathrm{NO}_{2}$ & $2.3(-12)$ & 1 & \\
\hline $\mathrm{C} 59 \mathrm{O} 2+\mathrm{HO}_{2} \rightarrow \mathrm{HYAC}+\mathrm{GCO} 3+\mathrm{OH}$ & $2.4(-13) \exp (1300 / T)$ & 1,3 & $\mathrm{~N} 25$ \\
\hline $\mathrm{C} 59 \mathrm{O} 2+\mathrm{CH}_{3} \mathrm{O}_{2} \rightarrow \mathrm{HYAC}+\mathrm{GCO} 3+\mathrm{HCHO}+\mathrm{HO}_{2}$ & $9.2(-14)$ & 1 & \\
\hline $\mathrm{C} 59 \mathrm{O} 2+\mathrm{CH}_{3} \mathrm{CO}_{3} \rightarrow \mathrm{HYAC}+\mathrm{GCO} 3+\mathrm{CO}_{2}+\mathrm{CH}_{3} \mathrm{O}_{2}$ & $1.8(-12) \exp (500 / T)$ & 6,7 & \\
\hline $\mathrm{ISOPBOH}+\mathrm{OH} \rightarrow \mathrm{DHBO}+\mathrm{CO}$ & $3.85(-11)$ & 10 & N26 \\
\hline $\mathrm{ISOPDOH}+\mathrm{OH} \rightarrow 0.9 \mathrm{DHBO}+0.9 \mathrm{CO}+0.1 \mathrm{HCOC} 5+0.1 \mathrm{HO}_{2}$ & $7.38(-11)$ & 10 & N26 \\
\hline $\begin{array}{l}\mathrm{HPALD} 1+\mathrm{OH} \rightarrow 0.45 \mathrm{OH}+1.35 \mathrm{CO}_{2}+0.55 \mathrm{HCHO}+0.65 \mathrm{CH}_{3} \mathrm{CO}_{3} \\
\quad+0.2 \mathrm{MMAL}+0.15 \mathrm{MGLY}+0.15 \mathrm{CO}+0.1 \mathrm{GLY}\end{array}$ & $1.0(-11)$ & 5,3 & $\mathrm{~N} 27$ \\
\hline $\mathrm{HPALD} 1+\mathrm{OH} \rightarrow \mathrm{MVK}+\mathrm{OH}+0.5 \mathrm{CO}+0.5 \mathrm{CO}_{2}$ & $0.5(-11)$ & 5,3 & $\mathrm{~N} 27$ \\
\hline $\mathrm{HPALD} 1+\mathrm{OH} \rightarrow \mathrm{MVK}+\mathrm{OH}+\mathrm{CO}_{2}$ & $1.5(-11)$ & 5,3 & $\mathrm{~N} 27$ \\
\hline $\mathrm{HPALD} 1+\mathrm{OH} \rightarrow \mathrm{MVKOOH}+\mathrm{OH}+\mathrm{CO}$ & $1.4(-11)$ & 5,3 & $\mathrm{~N} 27$ \\
\hline $\mathrm{HPALD} 1+\mathrm{OH} \rightarrow \mathrm{ICHE}$ & $0.8(-11)$ & 5,3 & $\mathrm{~N} 27$ \\
\hline $\begin{array}{l}\mathrm{HPALD} 1+\mathrm{O}_{3} \rightarrow 0.35 \mathrm{MGLY}+0.27 \mathrm{GLY}+1.19 \mathrm{OH}+0.65 \mathrm{CO} \\
\quad+0.65 \mathrm{CH}_{3} \mathrm{CO}_{3}+0.08 \mathrm{H}_{2} \mathrm{O}_{2}+0.73 \mathrm{HPAC}\end{array}$ & $2.4(-17)$ & 1 & \\
\hline $\begin{array}{l}\mathrm{HPALD} 2+\mathrm{OH} \rightarrow 0.45 \mathrm{OH}+1.35 \mathrm{CO}_{2}+0.55 \mathrm{HCHO}+0.65 \mathrm{CH}_{3} \mathrm{CO}_{3} \\
\quad+0.2 \mathrm{MMAL}+0.15 \mathrm{MGLY}+0.15 \mathrm{CO}+0.1 \mathrm{GLY}\end{array}$ & $1.0(-11)$ & 5,3 & $\mathrm{~N} 28$ \\
\hline $\mathrm{HPALD} 2+\mathrm{OH} \rightarrow \mathrm{MACR}+\mathrm{OH}+0.5 \mathrm{CO}+0.5 \mathrm{CO}_{2}$ & $0.5(-11)$ & 5,3 & $\mathrm{~N} 28$ \\
\hline $\mathrm{HPALD} 2+\mathrm{OH} \rightarrow \mathrm{MACR}+\mathrm{OH}+\mathrm{CO}_{2}$ & $1.5(-11)$ & 5,3 & $\mathrm{~N} 28$ \\
\hline $\mathrm{HPALD} 2+\mathrm{OH} \rightarrow \mathrm{OH}+2 \mathrm{CO}+2 \mathrm{HO}_{2}+\mathrm{HPACET}$ & $0.8(-11)$ & 5,3 & $\mathrm{~N} 28$ \\
\hline $\mathrm{HPALD} 2+\mathrm{OH} \rightarrow \mathrm{ICHE}$ & $1.4(-11)$ & 5,3 & $\mathrm{~N} 28$ \\
\hline $\begin{array}{l}\text { HPALD } 2+\mathrm{O}_{3} \rightarrow 0.27 \mathrm{HPACET}+1.7 \mathrm{OH}+0.28 \mathrm{HO}_{2} \\
\quad+0.5 \mathrm{CO}+0.73 \mathrm{MGLY}+0.74 \mathrm{GLY}+0.02 \mathrm{CO}_{2}\end{array}$ & $2.4(-17)$ & 1 & \\
\hline $\mathrm{MMAL}+\mathrm{OH} \rightarrow \mathrm{MGLY}+\mathrm{HO}_{2}+2 \mathrm{CO}_{2}$ & $1.5(-12)$ & 1 & $\mathrm{~N} 29$ \\
\hline $\mathrm{DIHPMEK}+\mathrm{OH} \rightarrow 2 \mathrm{OH}+\mathrm{CH}_{3} \mathrm{CO}_{3}+\mathrm{CO}+\mathrm{HCHO}$ & $1.63(-11)$ & 1 & N30 \\
\hline DIHPMEK $+\mathrm{OH} \rightarrow \mathrm{OH}+$ HPKETAL & $1.28(-11)$ & 1 & \\
\hline $\begin{array}{l}\mathrm{HPKETAL}+\mathrm{OH} \rightarrow 0.6 \mathrm{OH}+\mathrm{CO}+0.6 \mathrm{MGLY} \\
\quad+0.4 \mathrm{CH}_{3} \mathrm{CO}_{3}+0.4 \mathrm{HO}_{2}\end{array}$ & $3.0(-11)$ & & N31 \\
\hline $\mathrm{HPDIAL}+\mathrm{OH} \rightarrow \mathrm{OH}+\mathrm{CO}+\mathrm{MGLY}$ & $3.0(-11)$ & & N32 \\
\hline $\begin{array}{l}\mathrm{NISOPO} 2+\mathrm{NO} \rightarrow 1.82 \mathrm{NO}_{2}+0.42 \mathrm{MVK}+0.04 \mathrm{MACR} \\
+1.54 \mathrm{HCHO}+0.18 \mathrm{NC} 4 \mathrm{CHO}+0.9 \mathrm{HO}_{2}+0.72 \mathrm{CO}\end{array}$ & $K_{\mathrm{RO} 2 \mathrm{NO}}$ & $1,15,3$ & N33 \\
\hline $\begin{array}{l}\mathrm{NISOPO} 2+\mathrm{NO}_{3} \rightarrow 1.82 \mathrm{NO}_{2}+0.42 \mathrm{MVK}+0.04 \mathrm{MACR} \\
\quad+1.54 \mathrm{HCHO}+0.18 \mathrm{NC} 4 \mathrm{CHO}+0.9 \mathrm{HO}_{2}+0.72 \mathrm{CO}\end{array}$ & $2.3(-12)$ & $1,15,3$ & \\
\hline $\begin{array}{l}\mathrm{NISOPO} 2+\mathrm{HO}_{2} \rightarrow 0.535 \mathrm{NISOPOOHD}+0.22 \mathrm{NISOPOOHB} \\
\quad+0.245 \mathrm{OH}+0.245 \mathrm{NO}_{2}+0.225 \mathrm{MVK}+0.02 \mathrm{MACR}+0.245 \mathrm{HCHO}\end{array}$ & $2.5(-13) \exp (1300 / T)$ & $1,15,3$ & \\
\hline $\begin{array}{l}\text { NISOPO } 2+\text { NISOPO } 2 \rightarrow 0.17 \mathrm{MVK}+0.11 \mathrm{MACR}+0.7 \mathrm{HCHO} \\
\quad+0.42 \mathrm{NO}_{2}+0.78 \mathrm{NC} 4 \mathrm{CHO}+0.36 \mathrm{HO}_{2}+0.28 \mathrm{CO} \\
\quad+0.59 \mathrm{ISOPCNO} 3+0.11 \mathrm{ISOPANO} 3+0.1 \mathrm{ISOPDNO} 3\end{array}$ & $2.0(-12)$ & 15,3 & N34 \\
\hline $\begin{array}{l}\mathrm{NISOPO} 2+\mathrm{CH}_{3} \mathrm{O}_{2} \rightarrow 0.08 \mathrm{MVK}+0.06 \mathrm{MACR}+0.95 \mathrm{HCHO} \\
\quad+0.21 \mathrm{NO}_{2}+0.39 \mathrm{NC} 4 \mathrm{CHO}+0.38 \mathrm{HO}_{2}+0.14 \mathrm{CO}+0.4 \mathrm{CH}_{3} \mathrm{OH} \\
\quad+0.29 \mathrm{ISOPCNO} 3+0.06 \mathrm{ISOPANO} 3+0.05 \mathrm{ISOPDNO} 3\end{array}$ & $7.5(-13)$ & 15,3 & N34 \\
\hline $\begin{array}{l}\mathrm{NISOPO} 2+\mathrm{CH}_{3} \mathrm{CO}_{3} \rightarrow 0.38 \mathrm{MVK}+0.05 \mathrm{MACR}+1.39 \mathrm{HCHO} \\
\quad+0.75 \mathrm{NO}_{2}+0.25 \mathrm{NC} 4 \mathrm{CHO}+0.81 \mathrm{HO}_{2}+0.64 \mathrm{CO}+0.9 \mathrm{CH}_{3} \mathrm{O}_{2} \\
\quad+0.9 \mathrm{CO}_{2}+0.1 \mathrm{CH}_{3} \mathrm{COOH}\end{array}$ & $2.0(-12) \exp (500 / T)$ & 15,3 & N34 \\
\hline $\begin{array}{l}\mathrm{NISOPO} 2+\mathrm{ISOPBO} 2 \rightarrow 0.71 \mathrm{MVK}+0.08 \mathrm{MACR}+1.33 \mathrm{HCHO} \\
\quad+0.47 \mathrm{NO}_{2}+0.53 \mathrm{NC} 4 \mathrm{CHO}+0.95 \mathrm{HO}_{2}+0.36 \mathrm{CO}+0.5 \mathrm{ISOPBOH}\end{array}$ & $7.5(-13)$ & 15,3 & N34 \\
\hline $\begin{array}{l}\text { NISOPO } 2+\text { ISOPDO } 2 \rightarrow 0.08 \mathrm{MVK}+0.26 \mathrm{MACR}+0.55 \mathrm{HCHO} \\
\quad+0.21 \mathrm{NO}_{2}+0.39 \mathrm{NC} 4 \mathrm{CHO}+0.38 \mathrm{HO}_{2}+0.14 \mathrm{CO}+0.4 \mathrm{ISOPDOH} \\
\quad+0.29 \mathrm{ISOPCNO}_{2}+0.06 \mathrm{ISOPANO} 3+0.05 \mathrm{ISOPDNO} 3+0.4 \mathrm{HCOC} 5\end{array}$ & $6.8(-12)$ & 15,3 & N34 \\
\hline $\mathrm{NISOPOOHD}+\mathrm{OH} \rightarrow \mathrm{NISOPO} 2$ & $3.4(-12) \exp (200 / T)$ & 3 & N35 \\
\hline $\mathrm{NISOPOOHD}+\mathrm{OH} \rightarrow \mathrm{OH}+\mathrm{NC} 4 \mathrm{CHO}$ & $7.5(-12) \exp (20 / T)$ & 3 & N35 \\
\hline $\begin{array}{l}\text { NISOPOOHD }+\mathrm{OH} \rightarrow 0.19 \mathrm{CO}+0.95 \mathrm{HO}_{2}+0.43 \mathrm{OH}+0.69 \mathrm{NOA} \\
\quad+0.19 \mathrm{HCHO}+0.5 \mathrm{HPAC}+0.07 \mathrm{HPACET}+0.07 \mathrm{ETHLN} \\
\quad+0.24 \mathrm{IHNE}\end{array}$ & $2.37(-11) \exp (390 / T)$ & 3 & N36 \\
\hline $\begin{array}{l}\text { NISOPOOHD }+\mathrm{O}_{3} \rightarrow 0.2 \mathrm{OH}+0.87 \mathrm{NOA} \\
\quad+0.13 \mathrm{HPACET}+0.84 \mathrm{HPAC}+0.16 \mathrm{ETHLN}\end{array}$ & $1.3(-17)$ & 15 & N37 \\
\hline
\end{tabular}


Table 2. Continued.

\begin{tabular}{|c|c|c|c|}
\hline Reaction & Rate & Ref. & Note \\
\hline $\mathrm{NISOPOOHB}+\mathrm{OH} \rightarrow \mathrm{NISOPO} 2$ & $3.4(-12) \exp (200 / T)$ & 3 & N38 \\
\hline $\begin{array}{l}\text { NISOPOOHB }+\mathrm{OH} \rightarrow 0.23 \mathrm{GLYALD}+0.47 \mathrm{NOA}+0.76 \mathrm{OH}+0.09 \mathrm{CO} \\
+0.33 \mathrm{HO}_{2}+0.09 \mathrm{HCHO}+0.15 \mathrm{HPAC}+0.04 \mathrm{HYAC} \\
\quad+0.04 \mathrm{ETHLN}^{-0.51 \mathrm{HNE}}\end{array}$ & $8.72(-12) \exp (390 / T)$ & 3 & N39 \\
\hline $\begin{array}{l}\mathrm{IHNE}+\mathrm{OH} \rightarrow 0.23 \mathrm{HMVK}+0.03 \mathrm{HMAC}+0.82 \mathrm{HCHO}+0.8 \mathrm{NO}_{2} \\
\quad+0.8 \mathrm{CO}+0.17 \mathrm{NOA}+0.45 \mathrm{MGLY}+0.72 \mathrm{HO}_{2}+0.38 \mathrm{OH} \\
\quad+0.03 \mathrm{MVKNO}+0.09 \mathrm{HYAC}+0.09 \mathrm{CO}_{2}\end{array}$ & $3.22(-11) \exp (-400 / T)$ & 3 & $\mathrm{~N} 40$ \\
\hline $\begin{array}{l}\mathrm{NC} 4 \mathrm{CHO}+\mathrm{OH} \rightarrow 0.45 \mathrm{CO}_{2}+1.08 \mathrm{CO}+0.85 \mathrm{HO}_{2}+0.58 \mathrm{NOA}+0.5 \mathrm{OH} \\
\quad+0.12 \mathrm{HCHO}+0.12 \mathrm{MGLY}+0.17 \mathrm{NO}_{2}+0.11 \mathrm{MVKNO} 3 \\
\quad+0.05 \mathrm{ICHE}+0.14 \mathrm{CH}_{3} \mathrm{CO}_{3}+0.14 \mathrm{ETHLN}\end{array}$ & $4.1(-11)$ & 15,3 & N41 \\
\hline $\begin{array}{l}\mathrm{NC} 4 \mathrm{CHO}+\mathrm{NO}_{3} \rightarrow \mathrm{HNO}_{3}+\mathrm{CO}_{2}+0.75 \mathrm{NOA}+0.75 \mathrm{CO}+0.75 \mathrm{HO}_{2} \\
\quad+0.25 \mathrm{CH}_{3} \mathrm{CO}_{3}+0.25 \mathrm{ETHLN}\end{array}$ & $6.0(-12) \exp (-1860 / T)$ & 1,3 & N41 \\
\hline $\begin{array}{l}\mathrm{NC} 4 \mathrm{CHO}+\mathrm{O}_{3} \rightarrow 0.555 \mathrm{NOA}+0.89 \mathrm{CO}+0.89 \mathrm{OH}+0.445 \mathrm{MGLY} \\
\quad+0.445 \mathrm{HO}_{2}+0.075 \mathrm{H}_{2} \mathrm{O}_{2}+0.445 \mathrm{NO}_{2}+0.52 \mathrm{GLY} \\
\quad+0.035 \mathrm{OCHCOOH}^{-}\end{array}$ & $4.4(-18)$ & 1 & \\
\hline $\begin{array}{l}\text { ISOPCNO3 }+\mathrm{O}_{3} \rightarrow 0.555 \mathrm{NOA}+0.52 \mathrm{GLYALD}+0.07 \mathrm{C}_{2} \mathrm{H}_{5} \mathrm{COOH} \\
\quad+0.075 \mathrm{H}_{2} \mathrm{O}_{2}+0.89 \mathrm{OH}+0.445 \mathrm{NO}_{2}+0.445 \mathrm{MGLY} \\
\quad+0.445 \mathrm{HO}_{2}+0.445 \mathrm{CO}+0.445 \mathrm{HCHO}\end{array}$ & $2.8(-17)$ & 1,11 & \\
\hline $\mathrm{ISOPCNO} 3+\mathrm{OH} \rightarrow 1.2 \mathrm{OH}+1.2 \mathrm{CO}+\mathrm{HO}_{2}+0.6 \mathrm{NOA}+0.4 \mathrm{NC} 4 \mathrm{CHO}$ & $7.5(-12) \exp (20 / T)$ & 3 & $\mathrm{~N} 42$ \\
\hline $\mathrm{ISOPCNO} 3+\mathrm{OH} \rightarrow 0.92 \mathrm{INCO} 2+0.08 \mathrm{IEPOX}+0.08 \mathrm{NO}_{2}$ & $2.04(-11) \exp (390 / T)$ & 3 & $\mathrm{~N} 43$ \\
\hline $\mathrm{INCO} 2 \rightarrow 4 \mathrm{HO}_{2}+2 \mathrm{CO}+\mathrm{OH}+\mathrm{NOA}$ & $1.256(13) \exp (-10000 / T)$ & 3 & N44 \\
\hline $\mathrm{INCO} 2+\mathrm{NO} \rightarrow \mathrm{INCNO} 3$ & $K_{\mathrm{RO} 2 \mathrm{NO}} \cdot Y_{\mathrm{nit}}(T, M, 11,4.7)$ & 3 & \\
\hline $\mathrm{INCO} 2+\mathrm{NO} \rightarrow \mathrm{NO}_{2}+\mathrm{HO}_{2}+\mathrm{NOA}+$ GLYALD & $K_{\mathrm{RO} 2 \mathrm{NO}} \cdot Y_{\mathrm{Oxy}}(T, M, 11,4.7)$ & 3 & N43 \\
\hline $\mathrm{INCO} 2+\mathrm{NO}_{3} \rightarrow \mathrm{NO}_{2}+\mathrm{HO}_{2}+\mathrm{NOA}+$ GLYALD & $2.3(-12)$ & 1 & N43 \\
\hline $\begin{array}{l}\mathrm{INCO} 2+\mathrm{HO}_{2} \rightarrow 0.32 \mathrm{INCCO}+0.11 \mathrm{INCO} 2+0.57 \mathrm{NOA}+0.57 \mathrm{GLYALD} \\
\quad+0.57 \mathrm{HO}_{2}+0.46 \mathrm{OH}\end{array}$ & $2.5(-13) \exp (1300 / T)$ & 3 & N45 \\
\hline $\mathrm{INCCO}+\mathrm{OH} \rightarrow \mathrm{HCHO}+3 \mathrm{HO}_{2}+\mathrm{CH}_{3} \mathrm{CO}_{3}+2 \mathrm{CO}+\mathrm{NO}_{2}$ & $3.3(-12)$ & 1 & N46 \\
\hline $\begin{array}{l}\mathrm{INCNO} 3+\mathrm{OH} \rightarrow 0.445 \mathrm{INCCO}+0.414 \mathrm{GLY}+0.414 \mathrm{HO}_{2} \\
+0.555 \mathrm{NOA}+0.141 \mathrm{GLYALD}+\mathrm{NO}_{2}\end{array}$ & $1.98(-12)$ & 1 & N47 \\
\hline $\begin{array}{l}\text { ISOPANO3 }+\mathrm{O}_{3} \rightarrow 0.555 \mathrm{HYAC}+0.555 \mathrm{ETHLN}+0.89 \mathrm{OH} \\
\quad+0.445 \mathrm{NO}_{2}+0.445 \mathrm{GLY}+0.445 \mathrm{HO}_{2}+0.055 \mathrm{H}_{2} \mathrm{O}_{2}\end{array}$ & $2.8(-17)$ & 1,11 & \\
\hline $\begin{array}{l}\text { ISOPANO3 }+\mathrm{OH} \rightarrow 1.2 \mathrm{OH}+0.6 \mathrm{CO}+0.6 \mathrm{CH}_{3} \mathrm{CO}_{3}+0.6 \mathrm{ETHLN} \\
\quad+0.4 \mathrm{HO}_{2}+0.4 \mathrm{NC} 4 \mathrm{CHO}\end{array}$ & $7.5(-12) \exp (20 / T)$ & 3 & $\mathrm{~N} 42$ \\
\hline $\mathrm{ISOPANO}+\mathrm{OH} \rightarrow 0.96 \mathrm{INAO} 2+0.04 \mathrm{IEPOX}+0.04 \mathrm{NO}_{2}$ & $2.95(-11) \exp (390 / T)$ & 3 & $\mathrm{~N} 43$ \\
\hline $\mathrm{INAO} 2 \rightarrow 3 \mathrm{HO}_{2}+\mathrm{CO}+\mathrm{CH}_{3} \mathrm{CO}_{3}+\mathrm{OH}+\mathrm{ETHLN}$ & $5.092(12) \exp (-10000 / T)$ & 3 & N48 \\
\hline $\mathrm{INAO} 2+\mathrm{NO} \rightarrow \mathrm{HNO}_{3}$ & $K_{\mathrm{RO} 2 \mathrm{NO}} \cdot Y_{\mathrm{nit}}(T, M, 11,2.3)$ & 1 & N12 \\
\hline $\begin{array}{l}\text { INAO2 }+\mathrm{NO} \rightarrow 0.86 \mathrm{HYAC}+0.86 \mathrm{ETHLN}+0.14 \mathrm{MVKNO} 3 \\
\quad+0.14 \mathrm{HCHO}+\mathrm{HO}_{2}+\mathrm{NO}_{2}\end{array}$ & $K_{\mathrm{RO} 2 \mathrm{NO}} \cdot Y_{\text {оху }}(T, M, 11,2.3)$ & 3 & N43 \\
\hline $\begin{array}{l}\mathrm{INAO} 2+\mathrm{NO}_{3} \rightarrow 0.86 \mathrm{HYAC}+0.86 \mathrm{ETHLN}+0.14 \mathrm{MVKNO} 3 \\
\quad+0.14 \mathrm{HCHO}+\mathrm{HO}_{2}+\mathrm{NO}_{2}\end{array}$ & $2.3(-12)$ & 1 & N43 \\
\hline $\begin{array}{l}\mathrm{INAO} 2+\mathrm{HO}_{2} \rightarrow 0.32 \mathrm{CO}+0.64 \mathrm{HO}_{2}+0.33 \mathrm{OH}+0.18 \mathrm{INAO} 2 \\
\quad+0.44 \mathrm{HYAC}+0.44 \mathrm{ETHLN}+0.06 \mathrm{HCHO}+0.38 \mathrm{MVKNO} 3\end{array}$ & $2.6(-13) \exp (1300 / T)$ & 3 & N49 \\
\hline $\mathrm{HALD} 1+\mathrm{OH} \rightarrow \mathrm{CO}+2 \mathrm{OH}+\mathrm{CO}_{2}+0.5 \mathrm{CH}_{3} \mathrm{CO}_{3}+0.5 \mathrm{HMVK}$ & $1.5(-11)$ & & N50 \\
\hline HALD $1+\mathrm{OH} \rightarrow 0.65 \mathrm{IEPOXAO} 2+0.35 \mathrm{GLYALD}+0.35 \mathrm{MGLY}+0.35 \mathrm{HO}_{2}$ & $2.2(-11)$ & & N51 \\
\hline $\begin{array}{l}\mathrm{HALD} 1+\mathrm{NO}_{3} \rightarrow 2 \mathrm{CO}+\mathrm{CO}_{2}+3 \mathrm{OH}+\mathrm{HO}_{2}+\mathrm{CH}_{3} \mathrm{CO}_{3}+\mathrm{HNO}_{3} \\
\mathrm{HALD} 1+\mathrm{O}_{3} \rightarrow 0.55 \mathrm{GLYALD}+0.55 \mathrm{MGLY}+0.9 \mathrm{OH} \\
\quad+0.45 \mathrm{CO}+0.45 \mathrm{CH}_{3} \mathrm{CO}_{3}+0.45 \mathrm{HO}_{2}+0.45 \mathrm{GLY}\end{array}$ & $\begin{array}{l}5.6(-12) \exp (-1860 / T) \\
2.4(-17)\end{array}$ & $\begin{array}{r}\mathrm{N} 50 \\
1\end{array}$ & \\
\hline $\begin{array}{l}\mathrm{HALD} 2+\mathrm{OH} \rightarrow 0.5 \mathrm{CO}+1.5 \mathrm{OH}+0.5 \mathrm{CH}_{3} \mathrm{CO}_{3}+0.5 \mathrm{CO}_{2} \\
\quad+0.5 \mathrm{PGA}+0.5 \mathrm{HMAC}\end{array}$ & $1.5(-11)$ & & N50 \\
\hline $\mathrm{HALD} 2+\mathrm{OH} \rightarrow 0.35 \mathrm{IEPOXBO} 2+0.65 \mathrm{HYAC}+0.65 \mathrm{GLY}+0.65 \mathrm{HO}_{2}$ & $2.2(-11)$ & & N51 \\
\hline $\mathrm{HALD} 2+\mathrm{NO}_{3} \rightarrow \mathrm{CO}+2 \mathrm{OH}+\mathrm{CH}_{3} \mathrm{CO}_{3}+\mathrm{PGA}+\mathrm{HNO}_{3}$ & $5.6(-12) \exp (-1860 / T)$ & & N50 \\
\hline $\begin{array}{l}\mathrm{HALD} 2+\mathrm{O}_{3} \rightarrow 0.55 \mathrm{HYAC}+0.55 \mathrm{GLY}+0.9 \mathrm{OH}+0.9 \mathrm{HO}_{2} \\
\quad+0.9 \mathrm{CO}+0.05 \mathrm{H}_{2} \mathrm{O}_{2}+0.45 \mathrm{MGLY}\end{array}$ & $2.4(-17)$ & 1 & \\
\hline
\end{tabular}


Table 2. Continued.

\begin{tabular}{|c|c|c|c|}
\hline Reaction & Rate & Ref. & Note \\
\hline \multicolumn{4}{|l|}{$\mathrm{C}_{4}$ compounds } \\
\hline $\begin{array}{l}\mathrm{MACR}+\mathrm{OH} \rightarrow \mathrm{CO}+0.036 \mathrm{HPACET}+0.036 \mathrm{HO}_{2}+0.964 \mathrm{HYAC} \\
\quad+0.964 \mathrm{OH}\end{array}$ & $4.4(-12) \exp (380 / T)$ & 3 & N52 \\
\hline $\mathrm{MACR}+\mathrm{OH} \rightarrow \mathrm{MCO} 3$ & $2.7(-12) \exp (470 / T)$ & 3 & \\
\hline $\begin{array}{l}\mathrm{MACR}+\mathrm{O}_{3} \rightarrow 0.9 \mathrm{MGLY}+0.12 \mathrm{HCHO}+0.1 \mathrm{CO}+0.1 \mathrm{OH} \\
\quad+0.1 \mathrm{CH}_{3} \mathrm{CO}_{3}+0.88\left(0.55 \mathrm{HMHP}+0.4 \mathrm{HCHO}+0.4 \mathrm{H}_{2} \mathrm{O}_{2}\right. \\
\quad+0.05 \mathrm{HCOOH})\end{array}$ & $1.4(-15) \exp (-2100 / T)$ & 1 & $\mathrm{~N} 2$ \\
\hline $\mathrm{MACR}+\mathrm{NO}_{3} \rightarrow \mathrm{MCO} 3+\mathrm{HNO}_{3}$ & $3.4(-15)$ & 1 & \\
\hline $\begin{array}{l}\mathrm{MCO} 3+\mathrm{NO} \rightarrow \mathrm{CO}_{2}+0.65 \mathrm{CH}_{3} \mathrm{O}_{2}+0.65 \mathrm{CO}+0.35 \mathrm{CH}_{3} \mathrm{CO}_{3} \\
\quad+\mathrm{HCHO}+\mathrm{NO}_{2}\end{array}$ & $8.70(-12) \exp (290 / T)$ & 1 & \\
\hline $\begin{array}{l}\mathrm{MCO} 3+\mathrm{NO}_{3} \rightarrow \mathrm{CO}_{2}+0.65 \mathrm{CH}_{3} \mathrm{O}_{2}+0.65 \mathrm{CO}+0.35 \mathrm{CH}_{3} \mathrm{CO}_{3} \\
\quad+\mathrm{HCHO}+\mathrm{NO}_{2}\end{array}$ & $4.0(-12)$ & 1 & \\
\hline $\mathrm{MCO} 3+\mathrm{HO}_{2} \rightarrow \mathrm{MCO} 3 \mathrm{H}$ & $2.43(-13) \exp (980 / T)$ & 1,18 & \\
\hline $\mathrm{MCO} 3+\mathrm{HO}_{2} \rightarrow \mathrm{MCOOH}+\mathrm{O}_{3}$ & $1.25(-13) \exp (980 / T)$ & 1,18 & \\
\hline $\begin{array}{l}\mathrm{MCO} 3+\mathrm{HO}_{2} \rightarrow \mathrm{CO}_{2}+0.65 \mathrm{CH}_{3} \mathrm{O}_{2}+0.65 \mathrm{CO}+0.35 \mathrm{CH}_{3} \mathrm{CO}_{3} \\
\quad+\mathrm{HCHO}+\mathrm{OH}\end{array}$ & $4.15(-13) \exp (980 / T)$ & 1,18 & \\
\hline $\begin{array}{l}\mathrm{MCO} 3+\mathrm{CH}_{3} \mathrm{O}_{2} \rightarrow 0.585 \mathrm{CH}_{3} \mathrm{O}_{2}+0.585 \mathrm{CO}+0.315 \mathrm{CH}_{3} \mathrm{CO}_{3} \\
\quad+1.9 \mathrm{HCHO}+0.9 \mathrm{HO}_{2}+0.9 \mathrm{CO}_{2}+0.1 \mathrm{MCOOH}\end{array}$ & $2.0(-12) \exp (500 / T)$ & $1,6,7$ & \\
\hline $\begin{array}{l}\mathrm{MCO} 3+\mathrm{CH}_{3} \mathrm{CO}_{3} \rightarrow 1.65 \mathrm{CH}_{3} \mathrm{O}_{2}+0.65 \mathrm{CO}+0.35 \mathrm{CH}_{3} \mathrm{CO}_{3} \\
\quad+\mathrm{HCHO}+2 \mathrm{CO}_{2}\end{array}$ & $5.4(-12) \exp (500 / T)$ & $1,6,7$ & \\
\hline $\begin{array}{l}\mathrm{MCO} 3+\mathrm{ISOPBO} 2 \rightarrow 0.65 \mathrm{CH}_{3} \mathrm{O}_{2}+0.65 \mathrm{CO}+0.35 \mathrm{CH}_{3} \mathrm{CO}_{3} \\
\quad+2 \mathrm{HCHO}+\mathrm{MVK}+\mathrm{HO}_{2}+\mathrm{CO}_{2}\end{array}$ & $1.8(-12) \exp (500 / T)$ & $1,6,7$ & \\
\hline $\begin{array}{l}\mathrm{MCO} 3+\mathrm{ISOPDO} 2 \rightarrow 0.585 \mathrm{CH}_{3} \mathrm{O}_{2}+0.585 \mathrm{CO}+0.315 \mathrm{CH}_{3} \mathrm{CO}_{3} \\
\quad+1.8 \mathrm{HCHO}+0.9 \mathrm{MACR}+0.9 \mathrm{HO}_{2}+0.9 \mathrm{CO}_{2} \\
\quad+0.1 \mathrm{MCOOH}+0.1 \mathrm{HCOC} 5\end{array}$ & $2.0(-12) \exp (500 / T)$ & $1,6,7$ & \\
\hline $\mathrm{MCO} 3+\mathrm{NO}_{2} \rightarrow \mathrm{MPAN}$ & $\begin{array}{l}k_{0}=3.28(-28)(300 / T)^{6.87} \\
k_{\infty}=1.125(-11)(300 / T)^{1.105}\end{array}$ & 1,19 & \\
\hline $\mathrm{MPAN} \rightarrow \mathrm{MCO} 3+\mathrm{NO}_{2}$ & $1.6(16) \exp (-13500 / T)$ & 1 & \\
\hline $\mathrm{MPAN}+\mathrm{OH} \rightarrow \mathrm{HYAC}+\mathrm{CO}+\mathrm{NO}_{3}$ & $7.5(-12)$ & 20 & \\
\hline $\mathrm{MPAN}+\mathrm{OH} \rightarrow \mathrm{HMML}+\mathrm{NO}_{3}$ & $2.25(-11)$ & 20 & \\
\hline $\mathrm{MPAN}+\mathrm{O}_{3} \rightarrow \mathrm{HCHO}+\mathrm{CH}_{3} \mathrm{CO}_{3}+\mathrm{NO}_{3}+\mathrm{CO}_{2}$ & $8.2(-18)$ & 1 & \\
\hline $\mathrm{MCO} 3 \mathrm{H}+\mathrm{OH} \rightarrow \mathrm{MCO} 3$ & $3.6(-12)$ & 1 & \\
\hline $\mathrm{MCO} 3 \mathrm{H}+\mathrm{OH} \rightarrow 0.83 \mathrm{HYAC}+0.83 \mathrm{CO}+0.17 \mathrm{HMML}+\mathrm{OH}$ & $1.3(-11)$ & 1 & \\
\hline $\begin{array}{l}\mathrm{MCOOH}+\mathrm{OH} \rightarrow \mathrm{CO}_{2}+0.65 \mathrm{CH}_{3} \mathrm{O}_{2}+0.65 \mathrm{CO} \\
\quad+0.35 \mathrm{CH}_{3} \mathrm{CO}_{3}+\mathrm{HCHO}\end{array}$ & $1.51(-11)$ & 1 & \\
\hline $\begin{array}{l}\mathrm{HMML}+\mathrm{OH} \rightarrow 1.13 \mathrm{CO}+1.05 \mathrm{OH}+0.39 \mathrm{HO}_{2}+0.48 \mathrm{CH}_{3} \mathrm{CHO} \\
\quad+0.87 \mathrm{CO}_{2}+0.44 \mathrm{CH}_{3} \mathrm{CO}_{3}+0.08 \mathrm{CH}_{3} \mathrm{COOH}\end{array}$ & $4.33(-12)$ & & N53 \\
\hline $\mathrm{MVK}+\mathrm{OH} \rightarrow \mathrm{MVKO} 2$ & $2.6(-12) \exp (610 / T)$ & 1 & \\
\hline $\begin{array}{l}\mathrm{MVK}+\mathrm{O}_{3} \rightarrow 0.313 \mathrm{CH}_{3} \mathrm{CO}_{3}+0.545 \mathrm{MGLY}+0.129 \mathrm{HO}_{2} \\
\quad+0.19 \mathrm{CO}+0.22 \mathrm{OH}+0.8 \mathrm{HCHO}+0.136 \mathrm{CH}_{3} \mathrm{CHO} \\
\quad+0.165 \mathrm{CO}_{2}+0.245 \mathrm{H}_{2} \mathrm{O}_{2}+0.275 \mathrm{HMHP} \\
\left.\quad+0.025 \mathrm{HCOOH}+0.006 \mathrm{CH}_{3} \mathrm{COOH}\right)\end{array}$ & $8.5(-16) \exp (-1520 / T)$ & 1 & N54 \\
\hline $\begin{array}{l}\mathrm{MVKO} 2+\mathrm{NO} \rightarrow 0.28 \mathrm{MGLY}+0.28 \mathrm{HCHO}+0.28 \mathrm{HO}_{2} \\
\quad+0.72 \mathrm{GLYALD}+0.72 \mathrm{CH}_{3} \mathrm{CO}_{3}+\mathrm{NO}_{2}\end{array}$ & $K_{\mathrm{RO} 2 \mathrm{NO}} \cdot Y_{\mathrm{oxy}}(T, M, 6,4.6)$ & $1,21,22$ & N55 \\
\hline $\mathrm{MVKO} 2+\mathrm{NO} \rightarrow \mathrm{MVKNO} 3$ & $K_{\mathrm{RO} 2 \mathrm{NO}} \cdot Y_{\mathrm{nit}}(T, M, 6,4.6)$ & 22 & \\
\hline $\begin{array}{l}\mathrm{MVKO} 2+\mathrm{NO}_{3} \rightarrow 0.28 \mathrm{MGLY}+0.28 \mathrm{HCHO}+0.28 \mathrm{HO}_{2} \\
\quad+0.72 \mathrm{GLYALD}+0.72 \mathrm{CH}_{3} \mathrm{CO}_{3}+\mathrm{NO}_{2}\end{array}$ & $2.3(-12)$ & 1 & N55 \\
\hline $\begin{array}{l}\mathrm{MVKO} 2+\mathrm{HO}_{2} \rightarrow 0.35 \mathrm{GLYALD}+0.35 \mathrm{CH}_{3} \mathrm{CO}_{3}+0.52 \mathrm{OH} \\
\quad+0.174 \mathrm{HO}_{2}+0.48 \mathrm{MVKOOH}+0.13 \mathrm{BIACETOH} \\
\quad+0.04 \mathrm{MGLY}+0.04 \mathrm{HCHO}\end{array}$ & $2.1(-13) \exp (1300 / T)$ & 22,3 & N55 \\
\hline $\begin{array}{l}\mathrm{MVKO} 2+\mathrm{CH}_{3} \mathrm{O}_{2} \rightarrow 0.14 \mathrm{MGLY}+0.36 \mathrm{GLYALD} \\
\quad+0.36 \mathrm{CH}_{3} \mathrm{CO}_{3}+0.89 \mathrm{HCHO}+0.64 \mathrm{HO}_{2}+0.25 \mathrm{DHBO} \\
\quad+0.18 \mathrm{BIACETOH}+0.07 \mathrm{HOBA}+0.25 \mathrm{CH}_{3} \mathrm{OH}\end{array}$ & $1.16(-12)$ & 1 & N55 \\
\hline $\begin{array}{l}\text { MVKO } 2+\mathrm{CH}_{3} \mathrm{CO}_{3} \rightarrow 0.25 \mathrm{MGLY}+0.65 \mathrm{GLYALD} \\
\quad+0.65 \mathrm{CH}_{3} \mathrm{CO}_{3}+0.25 \mathrm{HCHO}+0.25 \mathrm{HO}_{2}+0.9 \mathrm{CH}_{3} \mathrm{O}_{2} \\
\quad+0.9 \mathrm{CO}_{2}+0.1 \mathrm{CH}_{3} \mathrm{COOH}+0.1 \mathrm{DHBO}\end{array}$ & $2.0(-12) \exp (500 / T)$ & $1,6,7$ & \\
\hline
\end{tabular}


Table 2. Continued.

\begin{tabular}{|c|c|c|c|}
\hline Reaction & Rate & Ref. & Note \\
\hline $\mathrm{MVKOOH}+\mathrm{OH} \rightarrow 0.55 \mathrm{BIACETOH}+0.55 \mathrm{OH}+0.45 \mathrm{HOBA}$ & $4.5(-11)$ & 1 & N56 \\
\hline $\begin{array}{l}\mathrm{MACRNO} 3+\mathrm{OH} \rightarrow 0.5 \mathrm{HYAC}+0.5 \mathrm{MGLY}+0.5 \mathrm{HO}_{2}+0.5 \mathrm{CO} \\
\quad+0.5 \mathrm{CO}_{2}+\mathrm{NO}_{2}\end{array}$ & $3.0(-12)$ & 1 & N57 \\
\hline $\begin{array}{l}\mathrm{MVKNO}+\mathrm{OH} \rightarrow 0.5 \mathrm{BIACETOH}+0.4 \mathrm{GLY}+0.4 \mathrm{CH}_{3} \mathrm{CO}_{3} \\
\quad+0.1 \mathrm{MGLY}+0.1 \mathrm{CO}_{2}+0.5 \mathrm{HO}_{2}+\mathrm{NO}_{2}\end{array}$ & $1.76(-12)$ & 1 & N58 \\
\hline $\mathrm{MVKNO} 3+\mathrm{OH} \rightarrow \mathrm{HOBA}+\mathrm{NO}_{2}$ & $0.44(-12)$ & 1 & N58 \\
\hline $\mathrm{HOBA}+\mathrm{OH} \rightarrow 0.84 \mathrm{MGLY}+\mathrm{HO}_{2}+0.16 \mathrm{CH}_{3} \mathrm{CO}_{3}+0.32 \mathrm{CO}$ & $2.45(-11)$ & 1,14 & N59 \\
\hline $\mathrm{HOBA}+\mathrm{NO}_{3} \rightarrow \mathrm{HNO}_{3}+\mathrm{MGLY}+\mathrm{HO}_{2}$ & $5.6(-12) \exp (-1860 / T)$ & 1 & \\
\hline $\mathrm{DHBO}+\mathrm{OH} \rightarrow 0.61 \mathrm{BIACETOH}+0.39 \mathrm{HOBA}$ & $8.7(-12) \exp (70 / T)$ & 14 & \\
\hline $\begin{array}{l}\mathrm{MACROH}+\mathrm{OH} \rightarrow \mathrm{HO}_{2}+0.84 \mathrm{HYAC}+0.84 \mathrm{OH}+0.84 \mathrm{CO} \\
\quad-0.16 \mathrm{OH}+0.16 \mathrm{MGLY}+0.16 \mathrm{HO}_{2}+0.16 \mathrm{CO}_{2}\end{array}$ & $2.4(-11) \exp (70 / T)$ & 3 & N60 \\
\hline $\mathrm{BIACETOH}+\mathrm{OH} \rightarrow \mathrm{CH}_{3} \mathrm{CO}_{3}+2 \mathrm{CO}+\mathrm{HO}_{2}$ & $2.69(-12)$ & 14 & \\
\hline $\mathrm{HMVK}+\mathrm{OH} \rightarrow \mathrm{HCOOH}+\mathrm{OH}+\mathrm{MGLY}$ & $6.0(-11)$ & & N61 \\
\hline $\mathrm{HMVK}+\mathrm{OH} \rightarrow \mathrm{HO}_{2}+\mathrm{HOBA}$ & $2.4(-11)$ & & N61 \\
\hline $\begin{array}{l}\mathrm{HMAC}+\mathrm{OH} \rightarrow 0.5 \mathrm{HCOOH}+0.5 \mathrm{OH}+0.5 \mathrm{MGLY} \\
\quad+0.5 \mathrm{CO}+0.5 \mathrm{OH}+0.5 \mathrm{DHA}\end{array}$ & $3.0(-11)$ & & N62 \\
\hline $\begin{array}{l}\mathrm{HMAC}+\mathrm{OH} \rightarrow 0.89 \mathrm{CO}+1.34 \mathrm{OH}+0.78 \mathrm{CH}_{3} \mathrm{CO}_{3} \\
\quad+0.89 \mathrm{CO}_{2}+0.44 \mathrm{HO}_{2}+0.22 \mathrm{MGLY}\end{array}$ & $2.7(-11)$ & & N63 \\
\hline $\mathrm{HMAC}+\mathrm{NO}_{3} \rightarrow \mathrm{CO}+2 \mathrm{OH}+\mathrm{CH}_{3} \mathrm{CO}_{3}+\mathrm{CO}_{2}+\mathrm{HNO}_{3}$ & $3.4(-15)$ & & N63 \\
\hline \multicolumn{4}{|l|}{$\mathrm{C}_{3}$ compounds } \\
\hline $\mathrm{CH}_{3} \mathrm{COCH}_{3}+\mathrm{OH} \rightarrow \mathrm{ACETO}_{2}$ & $1.33(-13)+3.82(-11) \exp (-2000 / T)$ & 1 & \\
\hline $\mathrm{HPACET}+\mathrm{OH} \rightarrow \mathrm{MGLY}+\mathrm{OH}$ & $8.39(-12)$ & 1 & \\
\hline $\mathrm{HPACET}+\mathrm{OH} \rightarrow \mathrm{ACETO} 2$ & $1.9(-12) \exp (190 / T)$ & 1 & \\
\hline $\mathrm{ACETO} 2+\mathrm{NO} \rightarrow \mathrm{NO}_{2}+\mathrm{HCHO}+\mathrm{CH}_{3} \mathrm{CO}_{3}$ & $K_{\mathrm{RO} 2 \mathrm{NO}} \cdot Y_{\mathrm{Oxy}}(T, M, 4,5.2)$ & 1 & \\
\hline $\mathrm{ACETO} 2+\mathrm{NO} \rightarrow \mathrm{NOA}$ & $K_{\mathrm{RO} 2 \mathrm{NO}} \cdot Y_{\mathrm{nit}}(T, M, 4,5.2)$ & 1 & N64 \\
\hline $\mathrm{ACETO} 2+\mathrm{NO}_{3} \rightarrow \mathrm{NO}_{2}+\mathrm{HCHO}+\mathrm{CH}_{3} \mathrm{CO}_{3}$ & $2.3(-12)$ & 1 & \\
\hline $\begin{array}{l}\mathrm{ACETO} 2+\mathrm{HO}_{2} \rightarrow 0.85 \mathrm{HPACET} \\
\quad+0.15 \mathrm{HCHO}+0.15 \mathrm{CH}_{3} \mathrm{CO}_{3}\end{array}$ & $8.6(-13) \exp (700 / T)$ & 1,19 & \\
\hline $\begin{array}{l}\mathrm{ACETO} 2+\mathrm{CH}_{3} \mathrm{O}_{2} \rightarrow 0.3 \mathrm{CH}_{3} \mathrm{CO}_{3}+0.8 \mathrm{HCHO}+0.3 \mathrm{HO}_{2} \\
\quad+0.2 \mathrm{HYAC}+0.5 \mathrm{MGLY}+0.5 \mathrm{CH}_{3} \mathrm{OH}\end{array}$ & $3.8(-12)$ & 7 & \\
\hline $\mathrm{ACETO} 2+\mathrm{CH}_{3} \mathrm{CO}_{3} \rightarrow \mathrm{CH}_{3} \mathrm{COOH}+\mathrm{MGLY}$ & $2.5(-12)$ & 7 & \\
\hline $\mathrm{ACETO} 2+\mathrm{CH}_{3} \mathrm{CO}_{3} \rightarrow \mathrm{CH}_{3} \mathrm{O}_{2}+\mathrm{CO}_{2}+\mathrm{CH}_{3} \mathrm{CO}_{3}+\mathrm{HCHO}$ & $2.5(-12)$ & 7 & \\
\hline $\mathrm{ACETO} 2+\mathrm{ACETO} 2 \rightarrow \mathrm{HYAC}+\mathrm{MGLY}$ & $3.0(-12)$ & 7 & \\
\hline $\mathrm{ACETO} 2+\mathrm{ACETO} 2 \rightarrow 2 \mathrm{CH}_{3} \mathrm{CO}_{3}+2 \mathrm{HCHO}$ & $5.0(-12)$ & 7 & \\
\hline $\mathrm{HYAC}+\mathrm{OH} \rightarrow \mathrm{MGLY}+\mathrm{HO}_{2}$ & $1.46(-13) \exp (1100 / T) \cdot(T / 300)^{2.6}$ & 1,23 & \\
\hline $\mathrm{MGLY}+\mathrm{OH} \rightarrow 0.6 \mathrm{CH}_{3} \mathrm{CO}_{3}+0.4 \mathrm{CH}_{3} \mathrm{O}_{2}+1.4 \mathrm{CO}+\mathrm{H}_{2} \mathrm{O}$ & $1.9(-12) \exp (575 / T)$ & 1,24 & \\
\hline $\mathrm{MGLY}+\mathrm{NO}_{3} \rightarrow \mathrm{HNO}_{3}+\mathrm{CO}+\mathrm{CH}_{3} \mathrm{CO}_{3}$ & $3.36(-12) \exp (-1860 / T)$ & 1 & \\
\hline $\mathrm{NOA}+\mathrm{OH} \rightarrow \mathrm{MGLY}+\mathrm{NO}_{2}$ & $6.7(-13)$ & 1 & \\
\hline $\begin{array}{l}\mathrm{MVA}+\mathrm{OH} \rightarrow 0.5 \mathrm{CH}_{3} \mathrm{COOH}+0.5 \mathrm{HCHO}+0.5 \mathrm{OH} \\
\quad+0.5 \mathrm{HYAC}+0.5 \mathrm{HO}_{2}\end{array}$ & $9.0(-11)$ & & N65 \\
\hline $\begin{array}{l}\mathrm{DHA}+\mathrm{OH} \rightarrow 1.39 \mathrm{HO}_{2}+0.48 \mathrm{CH}_{3} \mathrm{CHO}+0.87 \mathrm{CO}_{2} \\
\quad+0.44 \mathrm{CH}_{3} \mathrm{CO}_{3}+0.08 \mathrm{CH}_{3} \mathrm{COOH}+0.13 \mathrm{CO}+0.05 \mathrm{OH}\end{array}$ & $8.0(-12) \exp (70 / T)$ & 3,19 & N66 \\
\hline \multicolumn{4}{|l|}{$\mathrm{C}_{2}$ compounds } \\
\hline $\mathrm{GLYALD}+\mathrm{OH} \rightarrow 0.78 \mathrm{GCO} 3+0.22 \mathrm{GLY}+0.22 \mathrm{HO}_{2}$ & $1.0(-11)$ & 1,25 & \\
\hline $\mathrm{GLYALD}+\mathrm{NO}_{3} \rightarrow \mathrm{GCO} 3+\mathrm{HNO}_{3}$ & $1.4(-12) \exp (-1860 / T)$ & 1 & \\
\hline $\mathrm{GCO} 3+\mathrm{NO} \rightarrow \mathrm{NO}_{2}+\mathrm{HO}_{2}+\mathrm{HCHO}+\mathrm{CO}_{2}$ & $6.7(-12) \exp (340 / T)$ & 1 & \\
\hline $\mathrm{GCO} 3+\mathrm{NO}_{3} \rightarrow \mathrm{NO}_{2}+\mathrm{HO}_{2}+\mathrm{HCHO}+\mathrm{CO}_{2}$ & $4.0(-12)$ & 1 & \\
\hline $\begin{array}{l}\mathrm{GCO} 3+\mathrm{HO}_{2} \rightarrow 0.21 \mathrm{GCO} 3 \mathrm{H}+0.04 \mathrm{GCOOH}+0.04 \mathrm{O}_{3} \\
\quad+0.75 \mathrm{HO}_{2}+0.75 \mathrm{HCHO}+0.75 \mathrm{OH}+0.75 \mathrm{CO}_{2}\end{array}$ & $7.84(-13) \exp (980 / T)$ & $1,17,26$ & \\
\hline $\mathrm{GCO} 3+\mathrm{CH}_{3} \mathrm{O}_{2} \rightarrow 1.9 \mathrm{HCHO}+1.8 \mathrm{HO}_{2}+0.1 \mathrm{GCOOH}+0.9 \mathrm{CO}_{2}$ & $1.8(-12) \exp (500 / T)$ & $1,6,7$ & \\
\hline $\mathrm{GCO} 3+\mathrm{CH}_{3} \mathrm{CO}_{3} \rightarrow \mathrm{CH}_{3} \mathrm{O}_{2}+\mathrm{HO}_{2}+\mathrm{HCHO}+2 \mathrm{CO}_{2}$ & $5.4(-12) \exp (500 / T)$ & $1,6,7$ & \\
\hline $\mathrm{GCO} 3+\mathrm{NO}_{2} \rightarrow \mathrm{GPAN}$ & $\begin{array}{l}k_{0}=3.28(-28)(300 / T)^{6.87} \\
k_{\infty}=1.125(-11)(300 / T)^{1.105}\end{array}$ & 1,19 & \\
\hline $\mathrm{GPAN} \rightarrow \mathrm{GCO} 3+\mathrm{NO}_{2}$ & $\begin{array}{l}k_{0}=1.1(-5) \exp (-10100 / T) \\
k_{\infty}=1.9(17) \exp (-14100 / T)\end{array}$ & 1,19 & \\
\hline
\end{tabular}


Table 2. Continued.

\begin{tabular}{|c|c|c|c|}
\hline Reaction & Rate & Ref. & Note \\
\hline $\mathrm{GPAN}+\mathrm{OH} \rightarrow \mathrm{HCHO}+\mathrm{CO}+\mathrm{NO}_{2}$ & $1.12(-12)$ & 1 & \\
\hline $\mathrm{GCO} 3 \mathrm{H}+\mathrm{OH} \rightarrow \mathrm{GCO} 3$ & $6.19(-12)$ & 1 & \\
\hline $\mathrm{GLY}+\mathrm{OH} \rightarrow 0.72 \mathrm{HO}_{2}+0.28 \mathrm{OH}+1.55 \mathrm{CO}+0.45 \mathrm{CO}_{2}$ & $3.1(-12) \exp (340 / T)$ & 1 & N67 \\
\hline $\mathrm{GLY}+\mathrm{NO}_{3} \rightarrow \mathrm{HNO}_{3}+0.72 \mathrm{HO}_{2}+0.28 \mathrm{OH}+1.55 \mathrm{CO}+0.45 \mathrm{CO}_{2}$ & $1.4(-12) \exp (-1860 / T)$ & 1 & N67 \\
\hline $\mathrm{HPAC}+\mathrm{OH} \rightarrow \mathrm{GLY}+\mathrm{OH}$ & $1.0(-11)$ & 1 & N68 \\
\hline $\mathrm{HPAC}+\mathrm{OH} \rightarrow 0.25 \mathrm{CO}+\mathrm{HCHO}+\mathrm{OH}+0.75 \mathrm{CO}_{2}$ & $1.8(-11)$ & 1 & N68 \\
\hline $\mathrm{HPAC}+\mathrm{OH} \rightarrow \mathrm{OCHCH}_{2} \mathrm{O}_{2}$ & $1.90(-12) \exp (190 / T)$ & 1 & \\
\hline $\mathrm{C}_{2} \mathrm{H}_{5} \mathrm{OH}+\mathrm{OH} \rightarrow 0.95 \mathrm{CH}_{3} \mathrm{CHO}+0.95 \mathrm{HO}_{2}+0.05 \mathrm{HOCH}_{2} \mathrm{CH}_{2} \mathrm{O}_{2}$ & $3.0(-12) \exp (20 / T)$ & 1 & \\
\hline $\mathrm{CH}_{3} \mathrm{CHO}+\mathrm{OH} \rightarrow 0.95 \mathrm{CH}_{3} \mathrm{CO}_{3}+0.05 \mathrm{OCHCH}_{2} \mathrm{O}_{2}$ & $4.7(-12) \exp (345 / T)$ & 1 & \\
\hline $\mathrm{CH}_{3} \mathrm{CHO}+\mathrm{NO}_{3} \rightarrow \mathrm{CH}_{3} \mathrm{CO}_{3}+\mathrm{HNO}_{3}$ & $1.4(-12) \exp (-1860 / T)$ & 1 & \\
\hline $\mathrm{OCHCH}_{2} \mathrm{O}_{2}+\mathrm{NO} \rightarrow \mathrm{NO}_{2}+\mathrm{HCHO}+\mathrm{CO}+\mathrm{HO}_{2}$ & $K_{\mathrm{RO} 2 \mathrm{NO}}$ & 1 & \\
\hline $\mathrm{OCHCH}_{2} \mathrm{O}_{2}+\mathrm{NO}_{3} \rightarrow \mathrm{NO}_{2}+\mathrm{HCHO}+\mathrm{CO}+\mathrm{HO}_{2}$ & $2.3(-12)$ & 1 & \\
\hline $\mathrm{OCHCH}_{2} \mathrm{O}_{2}+\mathrm{HO}_{2} \rightarrow \mathrm{HPAC}$ & $1.4(-13) \exp (1300 / T)$ & 1,3 & \\
\hline $\begin{array}{l}\mathrm{OCHCH}_{2} \mathrm{O}_{2}+\mathrm{CH}_{3} \mathrm{O}_{2} \rightarrow 1.25 \mathrm{HCHO}+0.5 \mathrm{CO}+\mathrm{HO}_{2} \\
\quad+0.25 \mathrm{GLY}+0.25 \mathrm{CH}_{3} \mathrm{OH}+0.25 \mathrm{GLYALD}\end{array}$ & $2.0(-12)$ & 1,5 & \\
\hline $\mathrm{CH}_{3} \mathrm{CO}_{3}+\mathrm{NO} \rightarrow \mathrm{NO}_{2}+\mathrm{CH}_{3} \mathrm{O}_{2}+\mathrm{CO}_{2}$ & $7.5(-12) \exp (290 / T)$ & 1 & \\
\hline $\mathrm{CH}_{3} \mathrm{CO}_{3}+\mathrm{NO}_{3} \rightarrow \mathrm{NO}_{2}+\mathrm{CH}_{3} \mathrm{O}_{2}+\mathrm{CO}_{2}$ & $4.0(-12)$ & 1 & \\
\hline $\begin{array}{l}\mathrm{CH}_{3} \mathrm{CO}_{3}+\mathrm{HO}_{2} \rightarrow 0.31 \mathrm{PAA}+0.16 \mathrm{CH}_{3} \mathrm{COOH}+0.16 \mathrm{O}_{3} \\
\quad+0.53 \mathrm{CH}_{3} \mathrm{O}_{2}+0.53 \mathrm{OH}+0.53 \mathrm{CO}_{2}\end{array}$ & $7.84(-13) \exp (980 / T)$ & 1,18 & \\
\hline $\begin{array}{l}\mathrm{CH}_{3} \mathrm{CO}_{3}+\mathrm{CH}_{3} \mathrm{O}_{2} \rightarrow \mathrm{HCHO}+0.9 \mathrm{HO}_{2}+0.9 \mathrm{CH}_{3} \mathrm{O}_{2} \\
\quad+0.9 \mathrm{CO}_{2}+0.1 \mathrm{CH}_{3} \mathrm{COOH}\end{array}$ & $2.0(-12) \exp (500 / T)$ & 6,7 & \\
\hline $\mathrm{CH}_{3} \mathrm{CO}_{3}+\mathrm{CH}_{3} \mathrm{CO}_{3} \rightarrow 2 \mathrm{CH}_{3} \mathrm{O}_{2}+2 \mathrm{CO}_{2}$ & $2.9(-12) \exp (500 / T)$ & 6,7 & \\
\hline $\mathrm{CH}_{3} \mathrm{CO}_{3}+\mathrm{NO}_{2} \rightarrow \mathrm{PAN}$ & $\begin{array}{l}k_{0}=3.28(-28)(300 / T)^{6.87} \\
k_{\infty}=1.125(-11)(300 / T)^{1.105}\end{array}$ & 1,19 & \\
\hline $\mathrm{PAN} \rightarrow \mathrm{CH}_{3} \mathrm{CO}_{3}+\mathrm{NO}_{2}$ & $\begin{array}{l}k_{0}=1.1(-5) \exp (-10100 / T) \\
k_{\infty}=1.9(17) \exp (-14100 / T)\end{array}$ & 1,19 & \\
\hline $\mathrm{PAA}+\mathrm{OH} \rightarrow \mathrm{CH}_{3} \mathrm{CO}_{3}$ & $3.7(-12)$ & 1 & \\
\hline $\mathrm{CH}_{3} \mathrm{COOH}+\mathrm{OH} \rightarrow \mathrm{CH}_{3} \mathrm{O}_{2}+\mathrm{CO}_{2}$ & $3.15(-14) \exp (920 / T)$ & 1,19 & \\
\hline $\mathrm{ETHLN}+\mathrm{OH} \rightarrow \mathrm{HCHO}+\mathrm{NO}_{2}+\mathrm{CO}_{2}$ & $2.0(-12)$ & 1 & N69 \\
\hline $\mathrm{ETHLN}+\mathrm{NO}_{3} \rightarrow \mathrm{HCHO}+\mathrm{NO}_{2}+\mathrm{CO}_{2}$ & $1.4(-12) \exp (1860 / T)$ & 1 & \\
\hline $\begin{array}{l}\mathrm{VA}+\mathrm{OH} \rightarrow 0.64 \mathrm{HCOOH}+0.64 \mathrm{HCHO}+0.64 \mathrm{OH} \\
\quad+0.36 \mathrm{GLYALD}+0.36 \mathrm{HO}_{2}\end{array}$ & $6.8(-11)$ & 28 & N70 \\
\hline $\mathrm{PGA}+\mathrm{OH} \rightarrow \mathrm{CO}+\mathrm{CO}_{2}+\mathrm{OH}$ & $1.6(-11)$ & 1 & \\
\hline \multicolumn{4}{|l|}{$\mathrm{C}_{1}$ compounds } \\
\hline $\mathrm{CH}_{3} \mathrm{O}_{2}+\mathrm{NO} \rightarrow \mathrm{NO}_{2}+\mathrm{HCHO}+\mathrm{HO}_{2}$ & $2.8(-12) \exp (300 / T)$ & 19 & \\
\hline $\mathrm{CH}_{3} \mathrm{O}_{2}+\mathrm{NO} \rightarrow \mathrm{CH}_{3} \mathrm{ONO}_{2}$ & $2.8(-12) \exp (300 / T) \cdot Y_{\text {nit }}(T, M, 1,50)$. & 19 & N71 \\
\hline $\mathrm{CH}_{3} \mathrm{O}_{2}+\mathrm{NO}_{3} \rightarrow \mathrm{NO}_{2}+\mathrm{HCHO}+\mathrm{HO}_{2}$ & $1.2(-12)$ & 1 & \\
\hline $\mathrm{CH}_{3} \mathrm{O}_{2}+\mathrm{HO}_{2} \rightarrow 0.9 \mathrm{CH}_{3} \mathrm{OOH}+0.1 \mathrm{HCHO}$ & $4.1(-13) \exp (750 / T)$ & 19 & \\
\hline $\mathrm{CH}_{3} \mathrm{O}_{2}+\mathrm{CH}_{3} \mathrm{O}_{2} \rightarrow 2 \mathrm{HCHO}+2 \mathrm{HO}_{2}$ & $\begin{array}{l}9.5(-14) \exp (390 / T) \\
/(1+0.0382 \exp (1130 / T))\end{array}$ & 19 & \\
\hline $\mathrm{CH}_{3} \mathrm{O}_{2}+\mathrm{CH}_{3} \mathrm{O}_{2} \rightarrow \mathrm{HCHO}+\mathrm{CH}_{3} \mathrm{OH}$ & $\begin{array}{l}9.5(-14) \exp (390 / T) \\
/(1+26.2 \exp (-1130 / T))\end{array}$ & 19 & \\
\hline $\mathrm{CH}_{3} \mathrm{O}_{2}+\mathrm{O}_{3} \rightarrow \mathrm{HCHO}+\mathrm{HO}_{2}$ & $2.9(-16) \exp (-1000 / T)$ & 19 & \\
\hline $\mathrm{CH}_{3} \mathrm{O}_{2}+\mathrm{OH} \rightarrow 0.92 \mathrm{HCHO}+1.84 \mathrm{HO}_{2}+0.08 \mathrm{CH}_{3} \mathrm{OH}$ & $1.6(-10) \cdot\left(1-f_{\mathrm{stab}}\right)$ & $28-31$ & N72 \\
\hline $\mathrm{CH}_{3} \mathrm{O}_{2}+\mathrm{OH} \rightarrow \mathrm{CH}_{3} \mathrm{OOOH}$ & $1.6(-10) \cdot f_{\mathrm{stab}}$ & 31 & N72 \\
\hline $\mathrm{CH}_{3} \mathrm{OOOH}+\mathrm{OH} \rightarrow \mathrm{HCHO}+\mathrm{HO}_{2}$ & $2.2(-11)$ & 31 & \\
\hline $\mathrm{CH}_{3} \mathrm{OOOH} \rightarrow 0.2 \mathrm{CH}_{3} \mathrm{OH}+0.8 \mathrm{HCHO}+1.6 \mathrm{HO}_{2}$ & $1.1(14)(T / 300)^{3.5} \exp (-12130 / T)$ & 31 & \\
\hline $\mathrm{CH}_{3} \mathrm{OOOH}+\left(\mathrm{H}_{2} \mathrm{O}\right)_{2} \rightarrow \mathrm{CH}_{3} \mathrm{OH}$ & $3.0(-15) \exp (-2500 / T)$ & 31 & N73 \\
\hline $\mathrm{CH}_{3} \mathrm{OOH}+\mathrm{OH} \rightarrow 0.3 \mathrm{HCHO}+0.3 \mathrm{OH}+0.7 \mathrm{CH}_{3} \mathrm{O}_{2}$ & $3.8(-12) \exp (200 / T)$ & 19 & \\
\hline $\mathrm{CH}_{3} \mathrm{ONO}_{2}+\mathrm{OH} \rightarrow \mathrm{HCHO}+\mathrm{NO}_{2}$ & $8.0(-13) \exp (-1000 / T)$ & 19 & \\
\hline $\begin{array}{l}\mathrm{HMHP}+\mathrm{OH} \rightarrow 0.45 \mathrm{HCOOH}+0.45 \mathrm{OH} \\
\quad+0.55 \mathrm{HCHO}+0.55 \mathrm{HO}_{2}\end{array}$ & $1.3(-12) \exp (500 / T)$ & 3,32 & N74 \\
\hline $\mathrm{CH}_{3} \mathrm{OH}+\mathrm{OH} \rightarrow \mathrm{HCHO}+\mathrm{HO}_{2}$ & $2.9(-12) \exp (-345 / T)$ & 19 & \\
\hline $\mathrm{HCHO}+\mathrm{OH} \rightarrow \mathrm{CO}+\mathrm{HO}_{2}$ & $55(-12) \exp (125 / T)$ & 19 & \\
\hline $\mathrm{HCHO}+\mathrm{NO}_{3} \rightarrow \mathrm{CO}+\mathrm{HO}_{2}+\mathrm{HNO}_{3}$ & $5.8(-16)$ & 19 & \\
\hline $\mathrm{HCOOH}+\mathrm{OH} \rightarrow \mathrm{CO}_{2}+\mathrm{HO}_{2}$ & $4.5(-13)$ & 1 & \\
\hline
\end{tabular}


Table 2. Continued.

\begin{tabular}{|c|c|c|c|}
\hline Reaction & Rate & Ref. & Note \\
\hline \multicolumn{4}{|l|}{ oxidation of monoterpenes } \\
\hline $\begin{array}{l}\mathrm{APIN}+\mathrm{OH} \rightarrow \mathrm{APINOHO} 2+0.1 \mathrm{HCOOH}+1.3 \mathrm{HCHO} \\
\quad+\mathrm{CH}_{3} \mathrm{COCH}_{3}+0.2 \mathrm{GLY}+0.05 \mathrm{MGLY}\end{array}$ & $1.2(-11) \exp (440 / T)$ & 1 & N75 \\
\hline $\begin{array}{l}\mathrm{APIN}+\mathrm{O}_{3} \rightarrow \mathrm{APINO} 3 \mathrm{O} 2+0.15 \mathrm{OH}+0.1 \mathrm{HCOOH} \\
\quad+1.3 \mathrm{HCHO}+0.06 \mathrm{HMHP}+\mathrm{CH}_{3} \mathrm{COCH}_{3} \\
\quad+0.2 \mathrm{GLY}+0.05 \mathrm{MGLY}\end{array}$ & $8.05(-16) \exp (-640 / T)$ & 1 & N75 \\
\hline $\begin{array}{l}\mathrm{APIN}+\mathrm{NO}_{3} \rightarrow 0.74 \mathrm{NO}_{2}+0.26 \mathrm{APINONO} 2 \\
\quad+1.3 \mathrm{HCHO}+\mathrm{CH}_{3} \mathrm{COCH}_{3}+0.2 \mathrm{GLY}+0.05 \mathrm{MGLY}\end{array}$ & $1.2(-12) \exp (490 / T)$ & 1 & N75 \\
\hline APINOHO $2+\mathrm{NO} \rightarrow 0.74 \mathrm{NO}_{2}+0.26 \mathrm{APINONO} 2$ & $K_{\mathrm{RO} 2 \mathrm{NO}}$ & 1 & N76 \\
\hline $\mathrm{APINOHO} 2+\mathrm{NO}_{3} \rightarrow \mathrm{NO}_{2}$ & $2.3(-12)$ & 1 & \\
\hline APINOHO $2+\mathrm{HO}_{2} \rightarrow$ products & $2.6(-13) \exp (1300 / T)$ & 1 & \\
\hline $\mathrm{APINO} 3 \mathrm{O} 2+\mathrm{NO} \rightarrow 0.74 \mathrm{NO}_{2}+0.26 \mathrm{APINONO} 2$ & $K_{\mathrm{RO} 2 \mathrm{NO}}$ & 1 & N76 \\
\hline $\mathrm{APINO} 3 \mathrm{O} 2+\mathrm{NO}_{3} \rightarrow \mathrm{NO}_{2}$ & $2.3(-12)$ & 1 & \\
\hline $\mathrm{APINO} 3 \mathrm{O} 2+\mathrm{HO}_{2} \rightarrow$ products & $2.6(-13) \exp (1300 / T)$ & 1 & \\
\hline $\mathrm{APINONO} 2+\mathrm{OH} \rightarrow \mathrm{NO}_{2}$ & $4.5(-12)$ & 1 & \\
\hline \multicolumn{4}{|l|}{ MBO oxidation } \\
\hline $\mathrm{MBO}+\mathrm{OH} \rightarrow \mathrm{MBOO} 2$ & $8.1(-12)$ ex & 1 & \\
\hline $\begin{array}{l}\mathrm{MBO}+\mathrm{O}_{3} \rightarrow 0.308 \mathrm{HCHO}+0.992 \mathrm{CH}_{3} \mathrm{COCH}_{3}+1.31 \mathrm{HO}_{2} \\
+0.01 \mathrm{CH}_{3} \mathrm{CHO}+0.89 \mathrm{CO}_{2}+0.168 \mathrm{HMHP}^{2}+0.64 \mathrm{CO}\end{array}$ & $1.0(-17)$ & 1 & N77 \\
\hline $\mathrm{MBOO} 2+\mathrm{NO} \rightarrow \mathrm{MBONO} 3$ & $K_{\mathrm{RO} 2 \mathrm{NO}} \cdot Y_{\mathrm{nit}}(T, M, 7,2.4)$ & 1,34 & N78 \\
\hline $\begin{array}{l}\mathrm{MBOO} 2+\mathrm{NO} \rightarrow 0.67 \mathrm{GLYALD}+\mathrm{CH}_{3} \mathrm{COCH}_{3}+\mathrm{HO}_{2} \\
\quad+0.33 \mathrm{HCHO}+0.33 \mathrm{CO}_{2}+\mathrm{NO}_{2}\end{array}$ & $K_{\mathrm{RO} 2 \mathrm{NO}} \cdot Y_{\mathrm{oxy}}(T, M, 7,2.4)$ & 1 & N78 \\
\hline $\begin{array}{l}\mathrm{MBOO} 2+\mathrm{NO}_{3} \rightarrow 0.67 \mathrm{GLYALD}+\mathrm{CH}_{3} \mathrm{COCH}_{3}+\mathrm{HO}_{2} \\
\quad+0.33 \mathrm{HCHO}+0.33 \mathrm{CO}_{2}+\mathrm{NO}_{2}\end{array}$ & $2.3(-12)$ & 1 & N78 \\
\hline $\mathrm{MBOO} 2+\mathrm{HO}_{2} \rightarrow 0.67 \mathrm{CO}+\mathrm{CH}_{3} \mathrm{COCH}_{3}+2 \mathrm{HO}_{2}+1.33 \mathrm{CO}_{2}$ & $2.3(-13) \exp (1300 / T)$ & 1,3 & N79 \\
\hline $\begin{array}{l}\mathrm{MBONO} 3+\mathrm{OH} \rightarrow \mathrm{NO}_{2}+0.67 \mathrm{CO}+0.33 \mathrm{CO}_{2} \\
\quad+\mathrm{CH}_{3} \mathrm{COCH}_{3}+2 \mathrm{HO}_{2}\end{array}$ & $2.0(-12)$ & 1 & $\mathrm{~N} 80$ \\
\hline
\end{tabular}

The Berndt et al. (2019) results thus give the following product yields at $8 \mathrm{~s}$ : HPALDs $76 \%$, HPCE $15 \%$, dihydroperoxy carbonyls $2 \%$, while $5.5 \%$ of the reacted $Z$ - $\delta$-OH-peroxys are present as second-generation peroxys $\mathrm{C}_{5} \mathrm{H}_{9} \mathrm{O}_{5}$, and $1 \%$ are present as third-generation peroxys $\mathrm{C}_{5} \mathrm{H}_{9} \mathrm{O}_{7}$. The HPALD yield determined by Berndt et al. (2019) is much higher than that of Teng et al. (2017). However, another non-HPALD $\mathrm{C}_{5} \mathrm{H}_{8} \mathrm{O}_{3}$ compound observed by Teng et al. (2017) could be speculated to be a perhemiketale formed from HPALDs on the walls of the $1 \mathrm{~m}$ sampling tubing. Another observation of Berndt et al. (2019) indirectly supports a high HPALD yield. The concentration of the second-generation peroxys is strikingly high, given that the peroxys of type $i$ are expected to react at a rate of $\sim 2000 \mathrm{~s}^{-1}$ and those of type $i i$ even at $>$ $10^{4} \mathrm{~s}^{-1}$, such that at the given $Z-\delta-\mathrm{OH}$-peroxys concentrations and using the experimental $1,6 \mathrm{H}$-shift rates for $Z$ $\delta$-OH-peroxys I and II, they should be present in a quasisteady-state concentration of only about $10^{4} \mathrm{~cm}^{-3}$. This indicates that a large fraction of the $\mathrm{C}_{5} \mathrm{H}_{9} \mathrm{O}_{5}$ peroxys are $Z, E^{\prime}$ $\mathrm{HOCH}=\mathrm{C}\left(\mathrm{CH}_{3}\right)-\mathrm{CH}\left(\mathrm{O}_{2}\right)-\mathrm{CH}_{2} \mathrm{OOH}$ isomers of peroxy $i$ i (and similar for Case II) with the $\mathrm{OH}$ pointing outwards, away from the peroxy function, such that they cannot un- dergo the 1,6 enol-H-shift and can only be removed by (repeated ) $\mathrm{O}_{2}$ loss and re-addition to finally convert to $Z, E^{\prime}$ $\mathrm{HOCH}\left(\mathrm{O}_{2}\right) \mathrm{C}\left(\mathrm{CH}_{3}\right)=\mathrm{CHCH}_{2} \mathrm{OOH}$ peroxys $i$ that quickly expel $\mathrm{HO}_{2}$ to form additional HPALDs. A high fraction of $Z, E^{\prime}$ peroxys $i i$ is consistent with the computational results (Peeters et al., 2014) on the various transition states (TSs) for the $1,6 \mathrm{H}$-shift of the $Z-\delta$-OH-peroxys. For Case I, a $Z, Z^{\prime}-$ transition state with the $\mathrm{OH}$ inward was found to account for about $67 \%$ of the rate, and a $Z, E^{\prime}$-TS with $\mathrm{OH}$ outward accounts for $33 \%$, while for Case II two $Z, E^{\prime}$-TSs account for $69 \%$ and a $Z, Z^{\prime}$-TS for $31 \%$ of the rate. For the conditions of Berndt et al. (2019) at $8 \mathrm{~s}$, with the integrated 1,6 H-shift rate due for $\sim 92 \%$ to the Case II and $\sim 8 \%$ to the Case I $Z$ - $\delta$-OH-peroxys, the weighted average is $\sim 65 \%$ reaction through $Z, E^{\prime}$ and $35 \%$ through $Z, Z^{\prime}$ structures. Taken together, the above strongly suggests that, contrary to a speculative suggestion in the LIM1 paper, the $Z-E$ isomerism of the transition states is conserved in the allylic-radical products and in the resulting peroxys $i$ and $i i$. A statistical rate estimate for the prompt internal rotation of the $\mathrm{OH}$ in the $Z, E^{\prime}$-hydroxyl allyl product radicals, with computed barrier $12 \mathrm{kcal} \mathrm{mol}^{-1}$ and imaginary frequency close to $100 \mathrm{~cm}^{-1}$, and for a nascent vibration energy of $21 \mathrm{kcal} \mathrm{mol}^{-1}$, predicts 
Table 3. Photodissociation reactions. The last column gives the photolysis rate $(J)$ calculated using the TUV model (Madronich, 1993) for a zenith angle of $30^{\circ}$ and 300 DU ozone. References: 1, Burkholder et al. (2015); 2, Röth and Ehhalt (2015); 3, Shaw et al. (2018); 4, Pinho et al. (2005); 5, Jenkin et al. (2015); 6, Atkinson et al. (2006); 7, Liu et al. (2018); 8, Müller et al. (2014); 9, Barnes et al. (1993); 10, Xiong et al. (2016); 11, Liu et al. (2017); 12, Nakanishi et al. (1977); 13, Back and Yamamoto (1985).

\begin{tabular}{|c|c|c|c|c|}
\hline Reaction & Cross section & Quantum yield & Products & $J\left(\mathrm{~s}^{-1}\right)$ \\
\hline $\mathrm{HCHO} \rightarrow \mathrm{CO}+2 \mathrm{HO}_{2}$ & 1 & 2 & & $3.4(-5)$ \\
\hline $\mathrm{HCHO} \rightarrow \mathrm{H}_{2}+\mathrm{CO}$ & 1 & 2 & & $5.2(-5)$ \\
\hline $\mathrm{CH}_{3} \mathrm{CHO} \rightarrow \mathrm{CH}_{3} \mathrm{O}_{2}+\mathrm{CO}+\mathrm{HO}_{2}$ & 1 & 1 & & $5.0(-6)$ \\
\hline $\mathrm{CH}_{3} \mathrm{CHO} \rightarrow \mathrm{VA}$ & 1 & 3 & & $1.7(-6)$ \\
\hline $\begin{aligned} \text { GLYALD } & \stackrel{83 \%}{\longrightarrow} \mathrm{HCHO}+\mathrm{CO}+2 \mathrm{HO}_{2} \\
& \stackrel{10 \%}{\longrightarrow} \mathrm{CH}_{3} \mathrm{OH}+\mathrm{CO} \\
& \stackrel{7 \%}{\longrightarrow} \mathrm{OH}+\mathrm{OCHCH}_{2} \mathrm{O}_{2}\end{aligned}$ & 1 & 1 & & $1.2(-5)$ \\
\hline $\mathrm{GLY} \rightarrow 2 \mathrm{CO}+2 \mathrm{HO}_{2}$ & 1 & 1 & & $7.6(-5)$ \\
\hline $\mathrm{GLY} \rightarrow 2 \mathrm{CO}+\mathrm{H}_{2}$ & 1 & 1 & & $1.6(-5)$ \\
\hline $\mathrm{GLY} \rightarrow \mathrm{HCHO}+\mathrm{CO}$ & 1 & 1 & & $3.1(-5)$ \\
\hline $\mathrm{CH}_{3} \mathrm{COCH}_{3} \rightarrow \mathrm{CH}_{3} \mathrm{CO}_{3}+\mathrm{CH}_{3} \mathrm{O}_{2}$ & 1 & 1 & & $5.5(-7)$ \\
\hline $\mathrm{MGLY} \rightarrow \mathrm{CH}_{3} \mathrm{CO}_{3}+\mathrm{CO}+\mathrm{HO}_{2}$ & 1 & 1 & & $1.4(-4)$ \\
\hline $\begin{array}{l}\mathrm{MACR} \\
\stackrel{50 \%}{\stackrel{50 \%}{\longrightarrow}} \mathrm{MCO} 3+\mathrm{HO}_{2} \\
\stackrel{0}{\longrightarrow} 0.35 \mathrm{CH}_{3} \mathrm{CO}_{3}+\mathrm{HCHO}+1.65 \mathrm{CO}+0.65 \mathrm{CH}_{3} \mathrm{O}_{2}+\mathrm{HO}_{2}\end{array}$ & 1 & $4^{\mathrm{a}}$ & 5 & $2.1(-6)$ \\
\hline $\begin{aligned} & \mathrm{MVK} \stackrel{50 \%}{\longrightarrow} \mathrm{C}_{3} \mathrm{H}_{6}+\mathrm{CO} \\
& \stackrel{50 \%}{\longrightarrow} \mathrm{CH}_{3} \mathrm{CO}_{3}+\mathrm{HCHO}+\mathrm{CO}+\mathrm{HO}_{2}\end{aligned}$ & 1 & 1 & 5 & $4.5(-6)$ \\
\hline $\mathrm{CH}_{3} \mathrm{OOH} \rightarrow \mathrm{HCHO}+\mathrm{HO}_{2}+\mathrm{OH}$ & 1 & $1^{b}$ & & $5.6(-6)$ \\
\hline $\mathrm{HMHP} \rightarrow \mathrm{HCOOH}+\mathrm{OH}+\mathrm{HO}_{2}$ & 1 & $\mathrm{~b}$ & & $4.8(-6)$ \\
\hline $\mathrm{ISOPBOOH} \rightarrow \mathrm{MVK}+\mathrm{HCHO}+\mathrm{HO}_{2}+\mathrm{OH}$ & $1^{\mathrm{c}}$ & $\mathrm{b}$ & 5 & $5.6(-6)$ \\
\hline $\mathrm{ISOPDOOH} \rightarrow \mathrm{MACR}+\mathrm{HCHO}+\mathrm{HO}_{2}+\mathrm{OH}$ & $1^{\mathrm{c}}$ & $\mathrm{b}$ & 5 & $5.6(-6)$ \\
\hline $\mathrm{ISOPEOOH} \rightarrow \mathrm{MACR}+\mathrm{HCHO}+\mathrm{HO}_{2}+\mathrm{OH}$ & $1^{\mathrm{c}}$ & $\mathrm{b}$ & 5 & $5.6(-6)$ \\
\hline $\mathrm{MACROH} \rightarrow \mathrm{HYAC}+\mathrm{CO}+2 \mathrm{HO}_{2}$ & $6^{\mathrm{d}}$ & $6^{\mathrm{d}}$ & 5 & $6.2(-5)$ \\
\hline $\begin{aligned} \mathrm{MVKOOH} & \stackrel{45 \%}{\longrightarrow} \mathrm{CH}_{3} \mathrm{CO}_{3}+\mathrm{HO}_{2}+\mathrm{HPAC} \\
& \stackrel{55 \%}{\longrightarrow} \mathrm{CH}_{3} \mathrm{CO}_{3}+\mathrm{GLYALD}+\mathrm{OH}\end{aligned}$ & 7 & $7^{\mathrm{e}}$ & $5^{\mathrm{f}}$ & $1.3(-4)$ \\
\hline $\mathrm{CH}_{3} \mathrm{ONO}_{2} \rightarrow \mathrm{HCHO}+\mathrm{HO}_{2}+\mathrm{NO}_{2}$ & 1 & $1^{\mathrm{b}}$ & & $9.0(-7)$ \\
\hline $\begin{aligned} & \mathrm{PAN} \stackrel{70 \%}{\longrightarrow} \mathrm{CH}_{3} \mathrm{CO}_{3}+\mathrm{NO}_{2} \\
& \stackrel{30 \%}{\longrightarrow} \mathrm{CH}_{3} \mathrm{O}_{2}+\mathrm{CO}_{2}+\mathrm{NO}_{3}\end{aligned}$ & 1 & $1^{\mathrm{b}}$ & & 7.3(-7) \\
\hline $\mathrm{PAA} \rightarrow \mathrm{CH}_{3} \mathrm{O}_{2}+\mathrm{OH}+\mathrm{CO}_{2}$ & 1 & $\mathrm{~b}$ & 5 & $7.9(-7)$ \\
\hline $\begin{aligned} \mathrm{HYAC} & \stackrel{50 \%}{\stackrel{50 \%}{\longrightarrow}} \mathrm{CH}_{3} \mathrm{CO}_{3}+\mathrm{HCHO}+\mathrm{HO}_{2} \\
& \stackrel{15 \%}{\longrightarrow} \mathrm{GCO}_{3}+\mathrm{CH}_{3} \mathrm{O}_{2} \\
& \stackrel{15 \%}{\longrightarrow} \mathrm{CH}+\mathrm{ACETO} 2\end{aligned}$ & 1 & 1 & 1 & $1.9(-6)$ \\
\hline $\mathrm{INDOOH} \rightarrow \mathrm{NO}_{2}+\mathrm{GLYALD}+\mathrm{HYAC}+\mathrm{OH}$ & $6^{\mathrm{g}}$ & $\mathrm{b}$ & $\mathrm{h}$ & $2.9(-6)$ \\
\hline $\begin{aligned} \mathrm{INDOOH} \rightarrow \mathrm{OH} & +0.15\left(\mathrm{HYAC}+\mathrm{GLYALD}+\mathrm{NO}_{2}\right) \\
& +0.85\left(\mathrm{HCHO}+\mathrm{HO}_{2}+\mathrm{MVKNO}\right)\end{aligned}$ & $1^{\mathrm{c}}$ & $\mathrm{b}$ & $\mathrm{i}$ & $5.6(-6)$ \\
\hline $\mathrm{MACRNO} 3 \rightarrow \mathrm{HYAC}+\mathrm{CO}+\mathrm{HO}_{2}+\mathrm{NO}_{2}$ & 8 & $8^{b}$ & 8 & $3.6(-4)$ \\
\hline $\begin{aligned} \mathrm{MVKNO} 3 & \rightarrow 0.8\left(\mathrm{CH}_{3} \mathrm{CO}_{3}+\mathrm{GLYALD}+\mathrm{NO}_{2}\right) \\
& +0.2\left(\mathrm{MGLY}+\mathrm{HCHO}+\mathrm{NO}_{2}\right)\end{aligned}$ & 8 & $8^{b}$ & 5 & $5.7(-5)$ \\
\hline $\mathrm{INCCO} \rightarrow \mathrm{NO}_{2}+\mathrm{HYAC}+\mathrm{GCO} 3$ & $6^{\mathrm{j}}$ & $8^{b}$ & 5 & $1.4(-5)$ \\
\hline $\mathrm{INCNO} 3 \rightarrow \mathrm{NO}_{2}+\mathrm{HCHO}+\mathrm{HO}_{2}+\mathrm{MVKNO} 3$ & $6^{\mathrm{k}}$ & $\mathrm{b}$ & $\mathrm{h}$ & $1.9(-6)$ \\
\hline $\mathrm{INCNO} 3 \rightarrow \mathrm{NO}_{2}+\mathrm{GLYALD}+\mathrm{NOA}+\mathrm{HO}_{2}$ & $6^{\mathrm{g}}$ & $\mathrm{b}$ & $\mathrm{h}$ & $2.9(-6)$ \\
\hline $\mathrm{NOA} \rightarrow \mathrm{CH}_{3} \mathrm{CO}_{3}+\mathrm{HCHO}+\mathrm{NO}_{2}$ & 9 & 8 & 5 & $3.2(-5)$ \\
\hline $\mathrm{ETHLN} \rightarrow \mathrm{HCHO}+\mathrm{CO}+\mathrm{HO}_{2}+\mathrm{NO}_{2}$ & 8 & 8 & 8 & $1.7(-4)$ \\
\hline $\begin{aligned} \mathrm{NC} 4 \mathrm{CHO} & \stackrel{16 \%}{\longrightarrow} \mathrm{NO}_{2}+1.15 \mathrm{HO}_{2}+1.35 \mathrm{CO}_{2}+0.55 \mathrm{HCHO} \\
& +0.65 \mathrm{CH}_{3} \mathrm{CO}_{3}+0.2 \mathrm{MMAL}+0.15 \mathrm{MGLY} \\
& +0.15 \mathrm{CO}+0.1 \mathrm{GLY}-0.55 \mathrm{OH}\end{aligned}$ & 10 & $10^{1}$ & $5^{\mathrm{m}}$ & $3.9(-4)$ \\
\hline $\mathrm{NC} 4 \mathrm{CHO} \stackrel{16 \%}{\longrightarrow} \mathrm{NO}_{2}+\mathrm{OH}+\mathrm{CO}+0.5 \mathrm{HPKETAL}+0.5 \mathrm{HPDIAL}$ & & & & \\
\hline
\end{tabular}


Table 3. Continued.

\begin{tabular}{|c|c|c|c|c|}
\hline Reaction & Cross section & Quantum yield & Products & $J\left(\mathrm{~s}^{-1}\right)$ \\
\hline \multicolumn{5}{|l|}{$\mathrm{NC} 4 \mathrm{CHO} \stackrel{48 \%}{\longrightarrow} \mathrm{NO}_{2}+\mathrm{CO}+\mathrm{OH}+0.3 \mathrm{HMVK}+0.7 \mathrm{HMAC}$} \\
\hline $\mathrm{NC} 4 \mathrm{CHO} \stackrel{20 \%}{\longrightarrow} \mathrm{NO}_{2}+1.7 \mathrm{CO}+0.3 \mathrm{MVKO} 2+0.7 \mathrm{HYAC}$ & & & & \\
\hline $\mathrm{DHBO} \rightarrow \mathrm{CH}_{3} \mathrm{CO}_{3}+$ GLYALD & 5 & 5 & 5 & $2.7(-6)$ \\
\hline $\mathrm{HOBA} \rightarrow \mathrm{MGLY}+\mathrm{CO}+2 \mathrm{HO}_{2}$ & $5^{\mathrm{n}}$ & $5^{\mathrm{n}}$ & 5 & $7.9(-6)$ \\
\hline $\mathrm{HOBA} \rightarrow \mathrm{CH}_{3} \mathrm{CO}_{3}+\mathrm{GLY}+\mathrm{HO}_{2}$ & $6^{\mathrm{n}}$ & $6^{\mathrm{n}}$ & & $1.9(-6)$ \\
\hline $\mathrm{HCOC} 5 \rightarrow \mathrm{CH}_{3} \mathrm{CO}_{3}+\mathrm{HCHO}+\mathrm{GCO} 3$ & 5 & 5 & 5 & $2.3(-6)$ \\
\hline $\mathrm{ICHE} \stackrel{28 \%}{\longrightarrow} 2 \mathrm{CO}+\mathrm{HO}_{2}+\mathrm{OH}+\mathrm{HYAC}$ & $6^{\mathrm{d}}$ & $6^{\mathrm{d}}$ & o & $6.2(-5)$ \\
\hline$\stackrel{72 \%}{\longrightarrow} \mathrm{CO}+\mathrm{HO}_{2}+\mathrm{MVKO} 2$ & & & o & \\
\hline $\mathrm{HPCE} \rightarrow \mathrm{HO}_{2}+1.82 \mathrm{CO}+0.82 \mathrm{OH}+0.82 \mathrm{HPACET}+0.18 \mathrm{KPO} 2$ & $6^{\mathrm{d}}$ & $6^{\mathrm{d}}$ & $\mathrm{p}$ & $6.2(-5)$ \\
\hline $\begin{array}{l}\mathrm{MCO} 3 \mathrm{H} \rightarrow \mathrm{OH}+\mathrm{CO}_{2}+0.65\left(\mathrm{CH}_{3} \mathrm{O}_{2}+\mathrm{CO}+\mathrm{HCHO}\right) \\
\quad+0.35\left(\mathrm{CH}_{3} \mathrm{CO}_{3}+\mathrm{HCHO}\right)\end{array}$ & $1^{q}$ & $\mathrm{~b}$ & 5 & $7.9(-7)$ \\
\hline $\mathrm{GCO} 3 \mathrm{H} \rightarrow \mathrm{OH}+\mathrm{HO}_{2}+\mathrm{HCHO}+\mathrm{CO}_{2}$ & $1^{\mathrm{q}}$ & $\mathrm{b}$ & 5 & $7.9(-7)$ \\
\hline $\mathrm{HPAC} \underset{16 \%}{\stackrel{84 \%}{\longrightarrow}}$ VA & 7 & $7^{\mathrm{e}}$ & $7^{\mathrm{r}}$ & $3.6(-4)$ \\
\hline $\begin{aligned} \stackrel{16 \%}{\longrightarrow} \mathrm{HO}_{2}+\mathrm{CO}+\mathrm{HCHO}+\mathrm{OH} \\
\text { HPACET } \stackrel{84 \%}{\stackrel{16 \%}{\longrightarrow}} \mathrm{MVA} \\
\stackrel{16 \%}{\longrightarrow} \mathrm{CH}_{3} \mathrm{CO}_{3}+\mathrm{HCHO}+\mathrm{OH}\end{aligned}$ & 7 & $7^{\mathrm{e}}$ & $7^{\mathrm{r}}$ & $1.3(-4)$ \\
\hline $\begin{aligned} \text { HPKETAL } & \stackrel{50 \%}{\longrightarrow} \mathrm{HMVK} \\
& \stackrel{25 \%}{\longrightarrow} \mathrm{CH}_{3} \mathrm{CO}_{3}+\mathrm{OH}+\mathrm{GLY} \\
& \stackrel{25 \%}{\longrightarrow} \mathrm{CO}+\mathrm{HO}_{2}+\mathrm{OH}+\mathrm{MGLY}\end{aligned}$ & 7 & $7^{\mathrm{e}}$ & $\mathrm{r}$ & $5.4(-4)$ \\
\hline $\begin{aligned} \text { HPDIAL } & \stackrel{50 \%}{\longrightarrow} \mathrm{HMAC} \\
& \stackrel{50 \%}{\longrightarrow} \mathrm{CO}+\mathrm{HO}_{2}+\mathrm{OH}+\mathrm{MGLY}\end{aligned}$ & 7 & $7^{\mathrm{e}}$ & $\mathrm{r}$ & $5.2(-4)$ \\
\hline DIHPMEK $\rightarrow \mathrm{OH}+\mathrm{CH}_{3} \mathrm{CO}_{3}+\mathrm{HPAC}$ & 7 & $7^{\mathrm{e}}$ & $5^{\mathrm{r}}$ & $1.3(-4)$ \\
\hline $\begin{aligned} & \text { BIACETOH } \stackrel{50 \%}{\longrightarrow} \mathrm{CH}_{3} \mathrm{CO}_{3}+\mathrm{GCO} 3 \\
& \stackrel{50 \%}{\longrightarrow} \mathrm{CH}_{3} \mathrm{CO}_{3}+\mathrm{CO}+\mathrm{HO}_{2}+\mathrm{HCHO}\end{aligned}$ & $6^{\mathrm{s}}$ & $6^{\mathrm{s}}$ & $\mathrm{t}$ & $7.1(-5)$ \\
\hline $\begin{aligned} \text { HPALD1 } & \stackrel{11 \%}{\longrightarrow} 0.45 \mathrm{OH}+1.15 \mathrm{HO}_{2}+1.35 \mathrm{CO}_{2}+0.55 \mathrm{HCHO} \\
+ & 0.65 \mathrm{CH}_{3} \mathrm{CO}_{3}+0.2 \mathrm{MMAL}+0.15 \mathrm{MGLY}+0.15 \mathrm{CO}+0.1 \mathrm{GLY} \\
& \stackrel{11 \%}{\longrightarrow} 2 \mathrm{OH}+\mathrm{CO}+\mathrm{HPKETAL} \\
& \stackrel{56 \%}{\longrightarrow} \mathrm{CO}+2 \mathrm{OH}+\mathrm{HMVK} \\
& \stackrel{22 \%}{\longrightarrow} \mathrm{CO}+\mathrm{CH}_{3} \mathrm{CO}_{3}+\mathrm{GLYALD}\end{aligned}$ & $1^{\mathrm{u}}$ & $\mathrm{u}$ & $11^{\mathrm{u}}$ & $4.2(-4)$ \\
\hline $\begin{aligned} \text { HPALD2 } & \stackrel{18 \%}{\longrightarrow} 0.45 \mathrm{OH}+1.15 \mathrm{HO}_{2}+1.35 \mathrm{CO}_{2}+0.55 \mathrm{HCHO} \\
+ & 0.65 \mathrm{CH}_{3} \mathrm{CO}_{3}+0.2 \mathrm{MMAL}+0.15 \mathrm{MGLY}+0.15 \mathrm{CO}+0.1 \mathrm{GLY} \\
& \stackrel{18 \%}{\longrightarrow} 2 \mathrm{OH}+\mathrm{CO}+\mathrm{HPKETAL} \\
& \stackrel{46 \%}{\longrightarrow} \mathrm{CO}+2 \mathrm{OH}+\mathrm{HMAC} \\
& \stackrel{18 \%}{\longrightarrow} 2 \mathrm{CO}+\mathrm{HO}_{2}+\mathrm{HYAC}\end{aligned}$ & $1^{\mathrm{u}}$ & $\mathrm{u}$ & $11^{\mathrm{u}}$ & $4.2(-4)$ \\
\hline $\mathrm{HMAC} \rightarrow \mathrm{OH}+\mathrm{CO}+\mathrm{HO}_{2}+\mathrm{MGLY}$ & 12 & $\mathrm{v}$ & $\mathrm{w}$ & $1.0(-5)$ \\
\hline $\mathrm{HMVK} \rightarrow \mathrm{OH}+\mathrm{CH}_{3} \mathrm{CO}_{3}+\mathrm{GLY}$ & 12 & $\mathrm{v}$ & w & $1.0(-5)$ \\
\hline $\mathrm{PGA} \rightarrow \mathrm{CO}+\mathrm{HO}_{2}+\mathrm{CO}_{2}+\mathrm{OH}$ & $\mathrm{x}$ & $\mathrm{x}$ & 5 & $1.1(-4)$ \\
\hline $\mathrm{APINONO} 2 \rightarrow \mathrm{NO}_{2}$ & $6^{\mathrm{g}}$ & $\mathrm{b}$ & & $2.9(-6)$ \\
\hline
\end{tabular}

Notes: ${ }^{\mathrm{a}}$ Total quantum yield of $0.004 .{ }^{\mathrm{b}}$ Unit quantum yield. ${ }^{\mathrm{c}}$ As for $\mathrm{CH}_{3} \mathrm{OOH}_{4}{ }^{\mathrm{d}}$ As for $\mathrm{i}-\mathrm{C}_{3} \mathrm{H}_{7} \mathrm{CHO}$. ${ }^{\mathrm{e}}$ Total quantum yield of $0.8 .{ }^{\mathrm{f}}$ See Sect. 2.1 .4 regarding hydroperoxycarbonyl photolysis, and Note $\mathrm{N} 56 .{ }^{\mathrm{g}} \mathrm{As}$ for $\mathrm{CH}_{3} \mathrm{CH}\left(\mathrm{ONO}_{2}\right) \mathrm{CH}_{3} .{ }^{\mathrm{h}} \mathrm{Oxy}$ radical decomposition follows Vereecken and Peeters (2009). ${ }^{\mathrm{i}}$ Oxy decomposition as in INDO2 + NO (Table 2). ${ }^{j}$ Sum of absorption cross sections of $\mathrm{CH}_{3} \mathrm{C}(\mathrm{O}) \mathrm{C}_{2} \mathrm{H}_{5}$ and $\mathrm{n}-\mathrm{C}_{4} \mathrm{H}_{9} \mathrm{ONO}_{2} \cdot{ }^{k} \mathrm{As} n-\mathrm{C}_{4} \mathrm{H}_{9} \mathrm{ONO}_{2} .{ }^{1}$ Quantum yield of 1 below $336 \mathrm{~nm}$, zero above (Xiong et al., 2016). ${ }^{\mathrm{m}}$ NC4CHO photolysis follows HPALD2 photolysis for $75 \%$ and HPALD1 for $25 \%$ (isomer distribution of Schwantes et al., 2015). ${ }^{\mathrm{n}}$ For the aldehyde channel, use $J\left(\mathrm{C}_{2} \mathrm{H}_{5} \mathrm{CHO}\right)$; for the ketone channel, use $J$ (HYAC). ${ }^{\circ} \mathrm{C}-\mathrm{C}$ scission leading to $\mathrm{HCO}$ and the same product radicals as in the formyl-H-abstraction pathway in ICHE+OH (Note N17). ${ }^{\mathrm{P}} \mathrm{C}-\mathrm{C}$ scission leading to $\mathrm{HCO}$ and the same product radicals as in the formyl-H-abstraction pathway in $\mathrm{HPCE}+\mathrm{OH}$ (Sect. 2.1.2). ${ }^{\mathrm{q}} \mathrm{As}$ for $\mathrm{CH}_{3} \mathrm{C}(\mathrm{O}) \mathrm{OOH}$. ${ }^{\mathrm{r}}$ See Sect. 2.1.4 regarding hydroperoxycarbonyl photolysis. ${ }^{\mathrm{s}}$ Photolysis rate taken as $25 \%$ of $J\left(\mathrm{CH}_{3} \mathrm{C}(\mathrm{O}) \mathrm{C}(\mathrm{O}) \mathrm{CH}_{3}\right)$ based on the experimental determination of Praske et al. (2015). ${ }^{\mathrm{t}}$ The reaction dominantly give $\mathrm{CH}_{3} \mathrm{C}^{\circ} \mathrm{O}+\mathrm{HOCH}_{2} \mathrm{C}^{\circ} \mathrm{O}$. The latter radical is formed with an internal energy ranging between 5 and

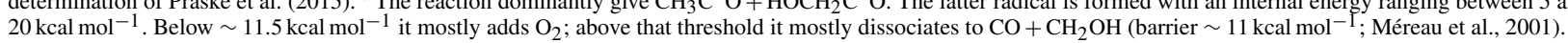

${ }^{\mathrm{u}}$ Absorption cross sections of MACR, quantum yield of 0.8. See Sect. 2.1.5 for the products. ${ }^{\mathrm{v}}$ Quantum yield of 0.1 below the threshold of $312 \mathrm{~nm}$ (see Sect. 2.1.5). ${ }^{\text {w }}$ See Sect. 2.1.5. ${ }^{\mathrm{x}}$ For peroxy glyoxylic acid, use the same photolysis parameters as for glyoxylic acid (Back and Yamamoto, 1985). The quantum yield is equal to 0.71. 

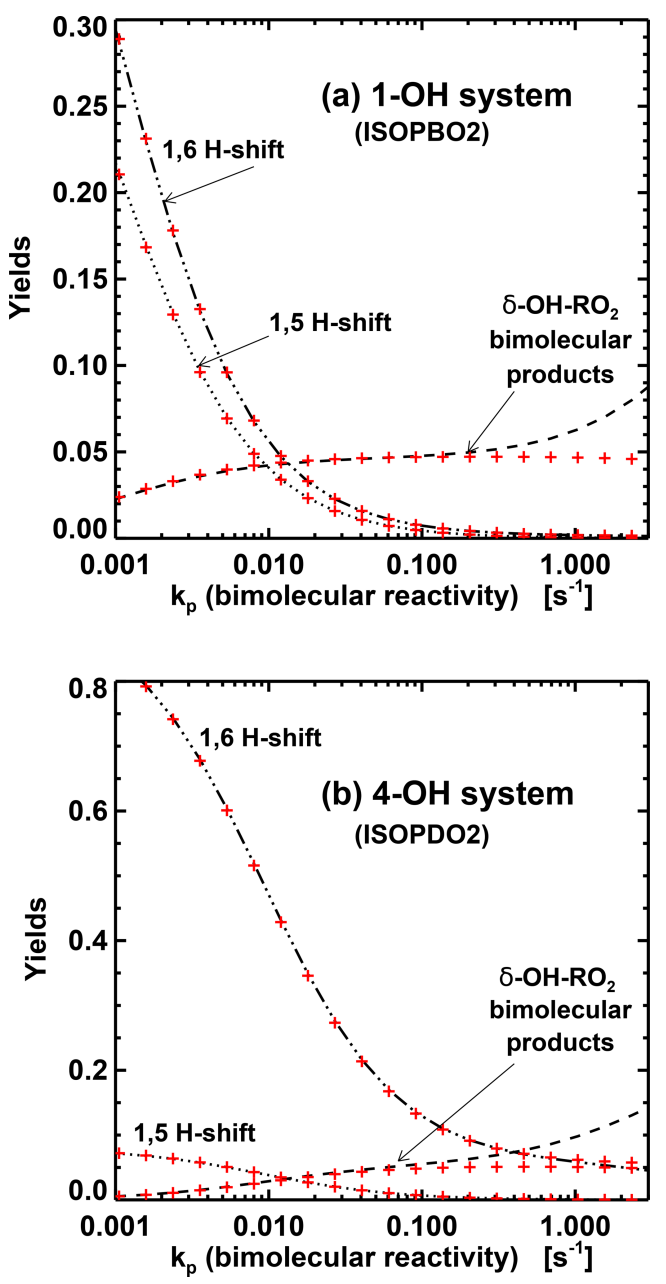

Figure 1. Contributions of $\mathrm{H}$-shift isomerizations and $\delta$-OH-peroxy bimolecular reactions to the total reactivity of isoprene peroxy radicals resulting from addition to $\mathrm{C}_{1}$ (a) and $\mathrm{C}_{4}$ (b), as a function of their bimolecular reactivity, at $295 \mathrm{~K}$ (Wennberg et al., 2018). The red crosses denote the yields of the parameterization used in the MAGRITTE mechanism.

$k \sim 10^{8} \mathrm{~s}^{-1}$, i.e. 10 times slower than collisional stabilization followed by $\mathrm{O}_{2}$ addition. Therefore, allowing for $10 \%$ internal rotation of the $\mathrm{OH}$ in the nascent $Z, E^{\prime}$ product isomers to form the more stable, H-bonded $Z, Z^{\prime}$ forms, about $40 \%$ of the allylic radicals and their $\mathrm{O}_{2}$-adducts would end up with the $\mathrm{OH}$ inwards and $\sim 60 \%$ with the $\mathrm{OH}$ outwards in the Berndt et al. (2019) conditions. Further, also adopting the spin densities in the allylic product radical of the LIM1 paper, i.e. 0.4 on $\mathrm{C}_{1}$ and 0.6 on $\mathrm{C}_{3}$ for Case I (and similarly 0.4 on $\mathrm{C}_{4}$ and 0.6 on $\mathrm{C}_{2}$ for Case II), as well as the corresponding $40: 60$ branching ratio for peroxy $i$ and $i i$ formation, the mechanism above would result in $40 \%$ direct formation of HPALDs through peroxy $i$, only $24 \%$ enol-H-shift products through $Z, Z^{\prime}$ peroxy $i i$, and $36 \%$ formation of the slowly reacting $Z, E^{\prime}$ peroxy $i i$, which in the Berndt et al. (2019) conditions would lead to ca. $31 \%$ indirect HPALD produc- tion through $\mathrm{O}_{2}$ loss and re-addition of the $Z, E^{\prime}$ peroxy $i i$ to form peroxy $i$, while around $5 \%$ still survives as $Z, E^{\prime}$ peroxy $i i$ in the short reaction time available. The thus-predicted overall $71 \%$ HPALD yield, based on computational results from the LIM1 paper, is strikingly close to the experimental yield of Berndt et al. (2019). Moreover, at a total product formation rate of $1.2 \times 10^{7} \mathrm{~cm}^{-3} \mathrm{~s}^{-1}$, the $31 \%$ contribution due to $Z, E^{\prime}$ peroxy $i i$ reacting to HPALDs at $8 \mathrm{~s}$ implies a reaction rate of $3.8 \times 10^{6} \mathrm{~cm}^{-3} \mathrm{~s}^{-1}$, or at the measured $Z, E^{\prime}$ peroxy $i$ concentration of $1.7 \times 10^{6} \mathrm{~cm}^{-3}$, an effective rate constant of $2.2 \mathrm{~s}^{-1}$. Since, on average, 2.5 cycles of $\mathrm{O}_{2}$ loss and re-addition are required to form HPALD from $Z, E^{\prime}$ peroxy $i i$ through peroxy $i$, an $\mathrm{O}_{2}$-loss rate of $6 \mathrm{~s}^{-1}$ is derived, which is typical for hydroxy-allyl peroxys such as the very similar initial $Z$ - and $E$ - $\delta$-OH-peroxys from isoprene (Teng et al., 2017).

The $15 \%$ HPCE yield measured by Berndt et al. (2019) is compatible with the product radical of the 1,6 enol- $\mathrm{H}-$ shift of $Z, Z^{\prime}$-peroxy $i i$ arising for a large fraction with sufficient chemical activation to overcome the barrier of ca. $15 \mathrm{kcal} \mathrm{mol}^{-1}$ for the concerted ring closure and $\mathrm{OH}$ loss. The theory-based $24 \%$ enol-H-shift products through peroxy $i i$, above, comprise the HPCE epoxides and products of the third-generation peroxys (DIHPCARP). Adopting the experimental $15 \%$ HPCE yield would leave room for some $10 \%$ DIHPCARP-derived products, of which, apparently, the dihydroperoxy carbonyls account for only a small fraction of $2 \%$. The minimum concentration of the DIHPCARPs in the Berndt et al. (2019) experiment is $3.5 \times 10^{5} \mathrm{~cm}^{-3}$, while their loss rate by aldehyde-H-shift (followed by either $\mathrm{CO}$ elimination or $\mathrm{O}_{2}$ addition) should be about $2 \mathrm{~s}^{-1}$ according to Møller et al. (2019) and $6 \mathrm{~s}^{-1}$ according to Novelli et al. (2018c), such that their expected reaction rate is $0.7-2.1 \times 10^{6} \mathrm{~cm}^{-3} \mathrm{~s}^{-1}$ or $6 \%-18 \%$ of the overall products formation rate of $1.2 \times 10^{7} \mathrm{~cm}^{-3} \mathrm{~s}^{-1}$ above. Subtracting the $2 \%$ dihydroperoxy carbonyls leaves $4 \%-16 \%$ going to other products, consistent with the $\sim 8 \%$ estimated above and in line with the expectation, in the introduction of this section, that the acyl product of aldehyde-H-shift in the most abundant DIHPCARP (Case II) does not eliminate $\mathrm{CO}$ but rather adds $\mathrm{O}_{2}$. The autoxidation chain is continued by forming fourth-generation peroxys $\mathrm{C}_{5} \mathrm{H}_{9} \mathrm{O}_{9}$, with $\quad \mathrm{HOOCH}_{2}-\mathrm{C}\left(\mathrm{CH}_{3}\right)\left(\mathrm{O}_{2}\right)-\mathrm{CH}(\mathrm{OOH})-\mathrm{C}(\mathrm{O}) \mathrm{OOH}$ (DHPAO2) likely the most stable isomer after fast hydroperoxide-H-shifts (Jørgensen et al., 2016) because it allows three H-bonds, of which two are synergic and, therefore, stronger (Dibble, 2004). Since (other) fast H-shifts for this isomer are not possible, it can only react with $\mathrm{NO}$ or $\mathrm{HO}_{2}$. The main resulting oxy product radical should decompose rapidly (Vereecken and Peeters, 2009) into $\mathrm{HPACET}+\mathrm{OH}+\mathrm{OCHC}(\mathrm{O}) \mathrm{OOH}$.

In atmospheric conditions, the various peroxys are all in quasi-steady state, which means $\sim 5 \%$ more HPALD production from the $Z, E^{\prime}$-peroxys $i i$ and $\sim 1 \%$ more DIHPCARP products than in the Berndt et al. (2019) conditions at 
$8 \mathrm{~s}$. On the other hand, the atmospheric steady-state product formation ratio from the $Z-\delta$-OH-peroxys Case I and Case II is $18: 82$, instead of the $8: 92$ ratio of the Berndt et al. (2019) experiment (Teng et al., 2017), such that about $43 \%$ of the second-generation radicals would end up with the $\mathrm{OH}$ inwards and $\sim 57 \%$ with the $\mathrm{OH}$ outwards. Also taking into account the above, direct (40\%) plus indirect (34\%) HPALD formation would add up to $74 \%$, while the expected HPCE yield is $16 \%$ and that of the DIHPCARP products around $10 \%$, of which $2 \%$ is the dihydroperoxy carbonyl DHPMEK. Acknowledging the large uncertainties in those yields, we represent the $Z-\delta-\mathrm{OH}$-peroxy isomerizations as follows:

$$
\begin{aligned}
& \text { ISOPBO } 2 \rightarrow 0.75\left(\mathrm{HPALD} 1+\mathrm{HO}_{2}\right)+0.15(\mathrm{HPCE}+\mathrm{OH}) \\
& \quad+0.1(\mathrm{DHPMEK}+\mathrm{CO}+\mathrm{OH}) \\
& \text { ISOPDO } 2 \rightarrow 0.75\left(\mathrm{HPALD} 2+\mathrm{HO}_{2}\right)+0.15(\mathrm{HPCE}+\mathrm{OH}) \\
& \quad+0.1(\mathrm{DHPAO} 2) .
\end{aligned}
$$

Here, HPCE is a mixture of $18 \%$ Case I and $82 \%$ Case II compounds. Its oxidation by $\mathrm{OH}$ proceeds mainly by aldehyde- $\mathrm{H}$ abstraction, forming a carbonyl radical; the same radical can also be formed through $\mathrm{OH}$ abstraction of the hydroperoxide-H in HPCE, followed by a 1,6 aldehyde-H-shift. The carbonyl radical can undergo concerted $\mathrm{CO}$ elimination and ring opening, forming $\mathrm{CH}_{3} \mathrm{C}(\mathrm{O}) \mathrm{CH}\left(\mathrm{O}_{2}\right) \mathrm{CH}_{2} \mathrm{OOH}$ (for Case I) or $\mathrm{OCHC}\left(\mathrm{O}_{2}\right)\left(\mathrm{CH}_{3}\right) \mathrm{CH}_{2} \mathrm{OOH}$ (for Case II). The latter peroxy undergoes a $1,4 \mathrm{H}$-shift to $\mathrm{CO}+\mathrm{OH}+\mathrm{CH}_{3} \mathrm{C}(\mathrm{O}) \mathrm{CH}_{2} \mathrm{OOH}$ (HPACET). As such a H-shift is not open for the Case I peroxy radical, it reacts primarily with $\mathrm{NO}$ or $\mathrm{HO}_{2}$, leading, for the most part, to $\mathrm{CH}_{3} \mathrm{C}(\mathrm{O}) \mathrm{CH}\left(\mathrm{O}^{\circ}\right) \mathrm{CH}_{2} \mathrm{OOH}$ that promptly decomposes into either $\mathrm{CH}_{3} \mathrm{C}(\mathrm{O})+\mathrm{OCHCH}_{2} \mathrm{OOH}$ (HPAC), or $\mathrm{HCHO}+\mathrm{OH}+\mathrm{MGLY}$. Photolysis of HPCE can be expected to proceed by splitting off the formyl radical, leading to the same peroxy radicals as above.

\subsubsection{Traditional chemistry of the initial $\delta$-OH-peroxy radicals}

The reactions of ISOPBO2 and ISOPDO2 with $\mathrm{NO}$ and $\mathrm{HO}_{2}$ generate a mixture of $\beta$ - and $\delta$-OH-peroxy reaction products. The share of the $\delta$-OH-peroxy reaction products is small (5\% for ISOPBO2 and $6 \%$ for ISOPDO2 at $297 \mathrm{~K}$ for a bimolecular peroxy lifetime of $50 \mathrm{~s}$ ) and assumed here to be constant. The absolute error on product yields due to this assumption does not exceed $0.5 \%$ in most atmospherically relevant conditions $\left(\mathrm{RO}_{2}\right.$ lifetime between 10 and $\left.100 \mathrm{~s}\right)$. As MAGRITTE is not intended to model local urban conditions, we omit the minor products of the bimolecular reactions of the $\delta$-hydroxyperoxy radicals, such as their reactions with other peroxy radicals. The hydroperoxides formed from their reactions with $\mathrm{HO}_{2}$ are lumped with the $\beta$-OH-counterparts, as are also the further-generation $\delta$-OH-epoxides. Besides nitrate formation, the reactions with $\mathrm{NO}$ form $Z$ - and $E-\delta$ $\mathrm{OH}$-allyloxy radicals that were shown (Nguyen and Peeters,
$2015)$ to interconvert rapidly and to react both in the $Z$ form by a fast $\alpha$-hydroxy-H-shift that leaves the products activated by a total of $32 \mathrm{kcal} \mathrm{mol}^{-1}$; this allows rotation of the $\mathrm{OH}$ in the hydroxy-allyl group over the barrier of $\sim 12 \mathrm{kcal} \mathrm{mol}^{-1}$ (Peeters et al., 2014) and, therefore, dominant formation of the more stable H-bonded $Z, Z^{\prime}$ form of the $\delta$-di-OH-allylic radicals: $\mathrm{HOC}^{\circ} \mathrm{HC}\left(\mathrm{CH}_{3}\right)=\mathrm{CHCH}_{2} \mathrm{OH}$ and $\mathrm{HOC}^{\circ} \mathrm{HCH}=\mathrm{C}\left(\mathrm{CH}_{3}\right) \mathrm{CH}_{2} \mathrm{OH}$. $\alpha$-addition of $\mathrm{O}_{2}$, for $45 \%$ (Teng et al., 2017), results in $\mathrm{C}_{5}$ hydroxyaldehydes HALD1 and HALD2 (4,1- and 1,4-HC5 in Wennberg et al. (2018) and HALD1 and HALD2 in the MCM) $+\mathrm{HO}_{2} \cdot \gamma$ addition of $\mathrm{O}_{2}$, for $55 \%$, results in $Z, Z^{\prime}$-enolperoxys, which were shown (Peeters and Nguyen, 2012) to undergo very fast 1,6 enol-H-shifts leading to nextgeneration peroxys that can isomerize by 1,4 aldehyde$\mathrm{H}$-shifts facing a barrier of only $20.2 \mathrm{kcal} \mathrm{mol}^{-1}$. Indeed, for 1,4 aldehyde- $\mathrm{H}$-shifts in similar hydroperoxy-formylperoxys with barriers of $20.6-21.2 \mathrm{kcal} \mathrm{mol}^{-1}$, rates of $\sim$ $1.5 \mathrm{~s}^{-1}$ were calculated and the products were shown to quickly lose $\mathrm{CO}$ and $\mathrm{OH}$ (Liu et al., 2017). Here, the expected products are $\mathrm{OH}+\mathrm{CO}+\mathrm{CH}_{3} \mathrm{C}(\mathrm{O}) \mathrm{CH}(\mathrm{OOH}) \mathrm{CH}_{2} \mathrm{OH}$ or $\mathrm{OCHC}\left(\mathrm{CH}_{3}\right)(\mathrm{OOH}) \mathrm{CH}_{2} \mathrm{OH}$. At very high $\mathrm{NO}$, as in some laboratory conditions, the $\mathrm{NO}$ reaction will dominate and yield either MGLY + GLYALD $+\mathrm{OH}$ or GLY $+\mathrm{HYAC}+\mathrm{OH}$, explaining these observed first-generation products (Paulot et al., 2009b; Galloway et al., 2011).

\subsubsection{Hydroperoxycarbonyl photolysis}

The isoprene oxidation mechanism generates several hydroperoxycarbonyls. Photolysis is expected to dominate the loss of all $\alpha$-hydroperoxyaldehydes (e.g. HPAC, $\mathrm{O}=\mathrm{CHCH}_{2} \mathrm{OOH}$ ) and several hydroperoxy ketones (e.g. HPACET, $\left.\mathrm{CH}_{3} \mathrm{C}(\mathrm{O}) \mathrm{CH}_{2} \mathrm{OOH}\right)$ due to estimated near-unit quantum yields and the strong enhancement of the absorption cross sections caused by the interaction between the hydroperoxy and carbonyl chromophores (Jorand et al., 2000; Liu et al., 2018). The expected likely major pathway in the photolysis of 2-hydroperoxy-propanal was theoretically determined to be a 1,5 $\mathrm{H}$-shift in the $\mathrm{S} 1$ state leading to enol formation (along with triplet $\mathrm{O}_{2}$ ) at an estimated yield of $84 \%$, whereas inter-system crossing (ISC) resulting in $\mathrm{C}-\mathrm{C}$ scission (i.e. formyl release) and $\mathrm{OH}$ expulsion makes up the rest (Liu et al., 2018). Similar yields are expected (and adopted here) for, e.g. HPAC and HPACET. However, the enol yield should be lower for heavier compounds due to expected faster ISC rates. It is taken to be $50 \%$ for, e.g. $\mathrm{CH}_{3} \mathrm{C}(\mathrm{O}) \mathrm{CH}(\mathrm{OOH}) \mathrm{CH}=\mathrm{O}(\mathrm{HPKETAL})$ and $\mathrm{O}=\mathrm{CHC}(\mathrm{OOH})\left(\mathrm{CH}_{3}\right) \mathrm{CH}=\mathrm{O}$ (HPDIAL). Furthermore, when $\mathrm{H}$-bonding between the carbonyl-O and the hydroperoxide- $\mathrm{H}$ supposed to undergo the $\mathrm{H}$-shift leading to enol formation is not favoured, e.g. because of possible H-bonds of this hydrogen with another oxygen in the molecule, enol formation is disadvantaged and, therefore, neglected here for simplicity. In those cases, formyl or acetyl loss, followed by $\mathrm{OH}$ expul- 
sion, is taken to be the only photolysis channel. Note that, to limit the number of compounds and reactions in the mechanism, several hydroperoxycarbonyls are not considered explicitly, and are replaced by their estimated photolysis products.

The theoretical investigation of the reaction of $\mathrm{OH}$ with vinyl alcohol (VA) (So et al., 2014) and propenols (Lei et al., 2018) is the basis for our evaluation of $\mathrm{OH}$ reactions with enols. $\mathrm{OH}$ addition generally follows; e.g.

$$
\begin{aligned}
& \mathrm{RCH}=\mathrm{CHOH}+\mathrm{OH}\left(+\mathrm{O}_{2}\right) \\
& \rightarrow \mathrm{RCH}\left(\mathrm{O}_{2}\right) \mathrm{CH}(\mathrm{OH})_{2} \stackrel{1,5 \mathrm{H}-\text { shift }}{\longrightarrow} \mathrm{HC}(\mathrm{O}) \mathrm{OH}+\mathrm{OH}+\mathrm{RCHO} \\
& \rightarrow \mathrm{RCH}(\mathrm{OH}) \mathrm{CH}(\mathrm{OH})_{2} \rightarrow \mathrm{HO}_{2}+\mathrm{RCH}(\mathrm{OH}) \mathrm{CHO} .
\end{aligned}
$$

In the case of vinyl alcohol (generated in HPAC photolysis), the formic acid yield is ca. $60 \%$, according to So et al. (2014). Acetic acid is similarly formed from the $\mathrm{OH}$ reaction of 2-propenol generated in the photolysis of hydroperoxyacetone (Lei et al., 2018). $\mathrm{HC}(\mathrm{O}) \mathrm{OH}$ should also be formed in the $\mathrm{OH}$ reaction of hydroxyvinyl methyl ketone ( $\mathrm{HMVK}, \mathrm{HOCH}=\mathrm{CHC}(\mathrm{O}) \mathrm{CH}_{3}$ ) and hydroxymethacrolein ( $\mathrm{HMAC}, \mathrm{O}=\mathrm{CHC}\left(\mathrm{CH}_{3}\right)=\mathrm{CHOH}$ ), although at a lower yield due to the competition with other possible reactions. Note that the acid-catalysed tautomerization of enols is neglected, based on the theoretical study of the case of vinyl alcohol (Peeters et al., 2015).

\subsubsection{HPALD photolysis}

The HPALD photolysis quantum yield is taken as equal to 0.8 , a compromise between the experimental value of $1 \pm 0.4$ for a $\mathrm{C}_{6}$ HPALD proxy (Wolfe et al., 2012) and the theoretical value (actually a lower limit) of 0.55 by Liu et al. (2017). The mechanism following HPALD photolysis is based on the theoretical study of Liu et al. (2017):

$$
\begin{aligned}
& \text { HPALD } 1+\mathrm{h} v \rightarrow \mathrm{OH}+0.11\left(\mathrm{HO}_{2}+\mathrm{O}=\mathrm{CHCH}=\mathrm{C}\left(\mathrm{CH}_{3}\right) \mathrm{CH}=\mathrm{O}(\mathrm{MBED})\right) \\
& +0.11\left(\mathrm{CO}+\mathrm{OH}+\mathrm{O}=\mathrm{CHCH}(\mathrm{OOH}) \mathrm{C}(\mathrm{O}) \mathrm{CH}_{3}(\mathrm{HPKETAL})\right) \\
& +0.56\left(\mathrm{CO}+\mathrm{OH}+\mathrm{O}=\mathrm{CHCH}=\mathrm{C}\left(\mathrm{CH}_{3}\right)(\mathrm{OH})(\mathrm{HMVK})\right) \\
& +0.22\left(\mathrm{CO}+\mathrm{CH}_{3} \mathrm{C}\left(\mathrm{O}_{2}\right)=\mathrm{CHCH}_{2} \mathrm{OH}^{\dagger}\left({\mathrm{V} 12^{\dagger}}^{\dagger}\right)\right) \\
& \mathrm{HPALD} 2+\mathrm{h} \nu \rightarrow \mathrm{OH}+0.18\left(\mathrm{HO}_{2}+\mathrm{O}=\mathrm{CHCH}=\mathrm{C}\left(\mathrm{CH}_{3}\right) \mathrm{CH}=\mathrm{O}(\mathrm{MBED})\right) \\
& +0.18\left(\mathrm{CO}+\mathrm{OH}+\mathrm{O}=\mathrm{CHCH}(\mathrm{OOH}) \mathrm{C}(\mathrm{O}) \mathrm{CH}_{3}(\mathrm{HPKETAL})\right) \\
& +0.46\left(\mathrm{CO}+\mathrm{OH}+\mathrm{O}=\mathrm{CHC}\left(\mathrm{CH}_{3}\right)=\mathrm{CHOH}(\mathrm{HMAC})\right) \\
& +0.18\left(\mathrm{CO}+\mathrm{HOCH}{ }_{2} \mathrm{C}\left(\mathrm{CH}_{3}\right)=\mathrm{CHO}_{2}^{\dagger}\left(\mathrm{V}_{2} \mathrm{O}^{\dagger}\right)\right) .
\end{aligned}
$$

Note that the formation of $\mathrm{OCHC}\left(\mathrm{CH}_{3}\right)(\mathrm{OOH}) \mathrm{CHO}$, considered in Wennberg et al. (2018) beside HPKETAL formation in the second photolysis channel of each HPALD, is neglected here as it was found to be minor (Liu et al., 2017).

Based on a reaction chamber study of butenedial and 4oxo-2-pentenal photolysis (Thuner et al., 2003), the photolysis of methylbutenedial (MBED) should be very fast (lifetime of minutes) and lead to a furanone-type compound as the major product, as well as methylmaleic anhydride (MMAL) and other compounds. Relying on MCM for the further oxidation of the furanone by $\mathrm{OH}$, we replace MBED by its assumed photooxidation products:

$$
\begin{aligned}
& \text { MBED } \stackrel{\text { fast }}{\longrightarrow} 0.55\left(-\mathrm{OH}+2 \mathrm{CO}_{2}+\mathrm{HCHO}+\mathrm{CH}_{3} \mathrm{CO}_{3}\right) \\
& \quad+0.20 \mathrm{MMAL}+0.15\left(\mathrm{MGLY}+\mathrm{CO}+\mathrm{HO}_{2}+\mathrm{CO}_{2}\right) \\
& \quad+0.10\left(\mathrm{GLY}+\mathrm{CH}_{3} \mathrm{CO}_{3}+\mathrm{CO}_{2}\right) .
\end{aligned}
$$

The major sink of the enols HMAC and HMVK should be their reaction with $\mathrm{OH}$, leading in part to formic acid formation (see Table 2). Based on the experimental study of Yoon et al. (1999), photolysis of the analogous ketone-enol form of acetylacetone $\left(\mathrm{CH}_{3} \mathrm{C}(\mathrm{O}) \mathrm{CH}=\mathrm{C}(\mathrm{OH}) \mathrm{CH}_{3}\right)$ yields $\mathrm{OH}$ and a vinylic co-product radical up to a wavelength of $312 \mathrm{~nm}$, with an $\mathrm{OH}$ appearance rate of $10^{8} \mathrm{~s}^{-1}$ or higher around $300 \mathrm{~nm}$, implying a quantum yield at atmospheric pressure of the order of 0.1 (instead of a near-unit quantum yield as assumed by Liu et al., 2017). The absorption cross sections of the enols are obtained from the acetylacetone study of Nakanishi et al. (1977). By analogy with the $\mathrm{CH}_{2}=\mathrm{CH}^{\circ}+\mathrm{O}_{2}$ reaction (Mebel and Kislov, 2005), we assume that the vinylic co-product radicals of HMAC and HMVK photolysis react rapidly with $\mathrm{O}_{2}$ to give $\mathrm{HCO}+\mathrm{MGLY}$ and $\mathrm{CH}_{3} \mathrm{CO}+\mathrm{GLY}$, respectively.

The activated vinylperoxy radicals $\mathrm{V}_{10}{ }^{\dagger}$ and $\mathrm{V} 2 \mathrm{O} 2^{\dagger}$ from HPALD photolysis might be stabilized by collisions and undergo reactions with $\mathrm{NO}, \mathrm{HO}_{2}$, and $\mathrm{NO}_{2}$ (Liu et al., 2017), but a more probable fate is decomposition (Mebel and Kislov, 2005) to $\mathrm{CH}_{3} \mathrm{CO}+\mathrm{GLYALD}$ in the case of $\mathrm{V} 1 \mathrm{O} 2$ and $\mathrm{HCO}+\mathrm{HYAC}$ in the case of $\mathrm{V} 2 \mathrm{O} 2$.

\subsection{Isoprene $+\mathrm{O}_{3}$}

The ozonolysis mechanism follows the experimentally derived model of Nguyen et al. (2016), except regarding the fate of the Criegee intermediate $\mathrm{CH}_{2} \mathrm{OO}$, formed with a yield of $58 \%$ (and assumed to be entirely stabilized). Whereas Nguyen et al. (2016) attributed a significant role to the reaction of $\mathrm{CH}_{2} \mathrm{OO}$ with the water monomer, motivated by the dependence of the observed yields on relative humidity, the reaction of $\mathrm{CH}_{2} \mathrm{OO}$ with the water dimer has been shown by several groups to be largely dominant at all relevant conditions (Berndt et al., 2014; Chao et al., 2015; Smith et al., 2015; Lewis et al., 2015; Sheps et al., 2017) and is, therefore, the only reaction considered here. More work is needed to elucidate the humidity dependence of the yields. Reaction with the dimer follows the recent study of Sheps et al. (2017):

$$
\begin{aligned}
& \mathrm{CH}_{2} \mathrm{OO}+\left(\mathrm{H}_{2} \mathrm{O}\right)_{2} \rightarrow 0.55\left(\mathrm{HOCH}_{2} \mathrm{OOH}+\mathrm{H}_{2} \mathrm{O}\right) \\
& \quad+0.4\left(\mathrm{HCHO}+\mathrm{H}_{2} \mathrm{O}_{2}+\mathrm{H}_{2} \mathrm{O}\right) \\
& \quad+0.05\left(\mathrm{HC}(\mathrm{O}) \mathrm{OH}+2 \mathrm{H}_{2} \mathrm{O}\right) .
\end{aligned}
$$

\subsection{Isoprene $+\mathrm{NO}_{3}$}

The mechanism for $\mathrm{NO}_{3}$-initiated oxidation largely follows the laboratory study of Schwantes et al. (2015). Several mi- 
nor pathways are neglected, however, as the further degradation mechanism of several products remain unclear. The title reaction, followed by $\mathrm{O}_{2}$ addition, forms several peroxy radical isomers lumped into one compound (NISOPO2). Generalizing the mechanism proposed by Schwantes et al. (2015), the reaction of NISOPO2 with non-tertiary peroxy radicals proceeds following

$$
\begin{aligned}
& \mathrm{NISOPO} 2+\mathrm{RO}_{2} \rightarrow 0.2\left(\mathrm{NISOPO}+\mathrm{RO}+\mathrm{O}_{2}\right) \\
& +0.4\left(0.88 \mathrm{NC} 4 \mathrm{CHO}+0.12 \mathrm{MACR}+0.12 \mathrm{HCHO}+0.12 \mathrm{NO}_{2}+\mathrm{ROH}\right) \\
& +0.4\left(0.74 \mathrm{ISOPCNO} 3+0.14 \mathrm{ISOPANO} 3+0.12 \mathrm{ISOPDNO} 3+\mathrm{R}^{\prime} \mathrm{CHO}\right),
\end{aligned}
$$

whereas for tertiary peroxy radicals the reaction reads

$$
\begin{aligned}
& \mathrm{NISOPO} 2+\mathrm{RO}_{2} \rightarrow 0.5\left(\mathrm{NISOPO}+\mathrm{RO}+\mathrm{O}_{2}\right) \\
& +0.5\left(0.88 \mathrm{NC} 4 \mathrm{CHO}+0.12 \mathrm{MACR}+0.12 \mathrm{HCHO}+0.12 \mathrm{NO}_{2}+\mathrm{ROH}\right) .
\end{aligned}
$$

The proposed 1,6 $\mathrm{H}$-shift of the trans-[1,4] isomer of NISOPO2 radicals (Schwantes et al., 2015) is neglected, as it is slow $\left(4 \times 10^{-4} \mathrm{~s}^{-1}\right)$ compared to the other reactions. The different isomers of the oxy radical NISOPO have different fates: decomposition to MVK or MACR (for the $\beta$ nitroxy oxys), reaction with $\mathrm{O}_{2}$ (for the $\delta$-nitroxyperoxys), and a fast 1,5 H-shift (Kwan et al., 2012) (ca. $2 \times 10^{5} \mathrm{~s}^{-1}$ ) for the $\delta$-(1-ONO $\left.\mathrm{ON}_{2}, 4-\mathrm{O}\right)$ radical, outrunning the $\mathrm{O}_{2}$ reaction by a factor of about 4 . The isomerization leads, after $\mathrm{O}_{2}$ addition, to a peroxy of which the reaction with $\mathrm{NO}$ or $\mathrm{NO}_{3}$ forms an enal nitrate, $\mathrm{O}_{2} \mathrm{NOCH}_{2} \mathrm{C}\left(=\mathrm{CH}_{2}\right) \mathrm{CH}=\mathrm{O}$, along with $\mathrm{HCHO}$ and $\mathrm{HO}_{2}$ (Wennberg et al., 2018). The main expected fate of this enal nitrate is photolysis, to $\mathrm{NO}_{2}+\mathrm{HCHO}+\mathrm{O}=\mathrm{CH}-\mathrm{C}\left(=\mathrm{CH}_{2}\right) \mathrm{O}_{2}$. The latter radical can undergo a fast $1,4 \mathrm{H}$-shift to give $\mathrm{CO}+\mathrm{OH}+\mathrm{H}_{2} \mathrm{C}=\mathrm{C}=\mathrm{O}$ (ketene). Ketene can react with $\mathrm{OH}$, at a rate of ca. $1.7 \times$ $10^{-11}$ molec. ${ }^{-1} \mathrm{~cm}^{3} \mathrm{~s}^{-1}$, producing $\mathrm{CO}+{ }^{\circ} \mathrm{CH}_{2} \mathrm{OH}$ (Calvert et al., 2011); it also photolyses to ${ }^{1} \mathrm{CH}_{2}\left(\right.$ or $\left.{ }^{3} \mathrm{CH}_{2}\right)+\mathrm{CO}$. The fate of methylene is mainly oxidation to $\mathrm{CO}$ or $\mathrm{CO}_{2}$ (Baulch et al., 2005). Based on photolysis parameter data provided by Calvert et al. (2011), photolysis is estimated to be slightly less important than the $\mathrm{OH}$ reaction, and is, therefore, neglected here for simplicity.

Based on the above, the lumped oxy radical undergoes the condensed fast reaction

$$
\begin{aligned}
& \mathrm{NISOPO} \rightarrow 0.42 \mathrm{MVK}+0.04 \mathrm{MACR}+1.54 \mathrm{HCHO} \\
& +0.82 \mathrm{NO}_{2}+0.18 \mathrm{NC} 4 \mathrm{CHO}+0.9 \mathrm{HO}_{2}+0.72 \mathrm{CO} .
\end{aligned}
$$

The $\beta$ - and $\delta$-nitroxy hydroperoxides formed in the $\mathrm{NISOPO} 2+\mathrm{HO}_{2}$ reaction are explicitly considered. Their reactions with $\mathrm{OH}$ form nitroxy hydroxy epoxides (IHNE), as well as hydroperoxy and nitroxy carbonyls, also explicitly considered in the mechanism. A major product of the NISOPO2 reaction with $\mathrm{NO}$ or $\mathrm{RO}_{2}$ is the enal nitrate $\mathrm{NC} 4 \mathrm{CHO}$. Laboratory work on an analogous compound (Xiong et al., 2016) has shown that photolysis is by far its dominant sink, owing to high quantum yields and to enhanced absorption cross sections attributed to the interaction of the nitrate and carbonyl chromophore. The NC4CHO photolysis cross sections and quantum yield recommendation follow Xiong et al. (2016). As the mechanism and products are uncertain (Xiong et al., 2016), we tentatively adopt a similar photolysis mechanism as for the analogous HPALDs but with $\mathrm{O}-\mathrm{NO}_{2}$ bond scission substituted for $\mathrm{O}-\mathrm{OH}$ scission. (see above, Sect. 2.1).

\subsection{Monoterpene oxidation}

Due to the complexity and poor understanding of monoterpene oxidation, we adopt a simple parameterization based on box model simulations of $\alpha$ - and $\beta$-pinene oxidation using the MCMv3.2 (Saunders et al., 2003). The scope of the parameterization is limited to the reproduction of total yields of several key products; those yields reflect not only primary production but also secondary formation. The influence of monoterpenes on radicals (e.g. $\mathrm{HO}_{x}, \mathrm{RO}_{2}$ ) and on ozone production is, therefore, likely not well represented by this simple mechanism. It should be stressed that even the monoterpene mechanism in MCM is greatly oversimplified, as it neglects many possibly important pathways (in particular $\mathrm{H}$ shift isomerizations in peroxy radicals), with potentially very large effects on radicals and other products. A thorough evaluation of mechanisms against laboratory data will be needed in order to assess their uncertainties but is out of scope of the present study.

The parameterization relies on simulations with lengths of $60 \mathrm{~d}$ performed using the Kinetic PreProcessor (KPP) package (Damian et al., 2002). The photolysis rates are calculated for clear-sky conditions at $30^{\circ} \mathrm{N}$ on 15 July. Although both high- $\mathrm{NO}_{x}\left(1 \mathrm{ppbv} \mathrm{NO}_{x}, 40 \mathrm{ppbv} \mathrm{O}_{3}\right.$, and $250 \mathrm{ppbv} \mathrm{CO}$ maintained throughout the simulation) and low- $\mathrm{NO}_{x}$ simulations ( 100 pptv $\mathrm{NO}_{x}, 20 \mathrm{ppbv} \mathrm{O}_{3}$, and $150 \mathrm{ppbv} \mathrm{CO}$ ) are conducted, only the low- $\mathrm{NO}_{x}$ results are used for the parameterization. Temperature and $\mathrm{H}_{2} \mathrm{O}$ are kept at $298 \mathrm{~K}$ and $1 \% v / v$. To determine the product yields, counter compounds are introduced in the equation file (e.g. HCHOa, MGLYOXa, etc.) that have the same production terms as the species they represent but without any chemical loss.

The yield of acetone from both $\alpha$ - and $\beta$-pinene is very close to $100 \%$ after several days of reaction, independent of the $\mathrm{NO}_{x}$ level. The yield of methylglyoxal is low (4\% and $5 \%$ for $\alpha$ - and $\beta$-pinene, not counting the contribution of acetone oxidation by $\mathrm{OH}$ ). The overall yield of formaldehyde obtained in these simulations is $\sim 4.2 \mathrm{HCHO}$ per monoterpene oxidized, almost independent of $\mathrm{NO}_{x}$, for both precursors. The $\mathrm{HCHO}$ yield comes down to 2.3 after subtracting the contributions of acetone and methylglyoxal oxidation. This yield is further reduced by $45 \%$ to account for wet/dry deposition of intermediates and secondary organic aerosol formation. That fraction is higher but of the same order as the estimated overall impact of deposition on the average formaldehyde yield from isoprene oxidation $(\sim 30 \%)$, based on global model (MAGRITTE) calculations. The higher frac- 
tion is justified by the larger number of oxidation steps and the generally lower volatility of intermediates involved in formaldehyde formation from monoterpene oxidation. Nevertheless, this adjustment introduces a significant uncertainty in the model results. A sensitivity calculation shows that adopting a lower yield reduction (20\% instead of $45 \%$ ) in the global model (Sect. 4.1) has negligible impact on the calculated $\mathrm{HCHO}$ abundances $(\lesssim 1 \%)$ in most regions but leads to higher $\mathrm{HCHO}$ vertical columns in monoterpene emission regions by $\sim 5 \%$ over Amazonia and by up to $8 \%$ over Siberia. The associated impact on $\mathrm{OH}$ reaches $+2 \%$ in those regions, due to the additional $\mathrm{HO}_{x}$ formation through $\mathrm{HCHO}$ photolysis.

The overall carbon balance of monoterpene oxidation in the mechanism is $\sim 50 \%$ due to the combined effects of deposition, $\mathrm{SOA}$ formation, and $\mathrm{CO}$ and $\mathrm{CO}_{2}$ formation besides their production through the degradation of the explicit products.

\subsection{Cross-reactions of peroxy radicals}

The channel ratios and rates of the cross reactions of peroxy radicals generally follow Capouet et al. (2004), except for the peroxy radicals from ISOP $+\mathrm{OH}$, for which we follow the recommendations of Wennberg et al. (2018) (based on measurements from Jenkin et al., 1998) and ISOP $+\mathrm{NO}_{3}$, based on Wennberg et al. (2018) and Schwantes et al. (2015). The cross reaction rates are calculated as twice the geometric mean of the self-reaction rates, except for acylperoxy radicals for which the rate and channel data reported for $\mathrm{CH}_{3} \mathrm{CO}_{3}$ are used (Atkinson et al., 2006). The self-reaction rates are obtained from compiled data for similar compounds (Capouet et al., 2004; Peeters and Müller, 2010; Atkinson et al., 2006).

\subsection{Peroxy radical reactions with $\mathrm{NO}$ and $\mathrm{HO}_{2}$}

We adopt the recommendations of Wennberg et al. (2018) for the rates of non-acyl peroxy radical reactions with $\mathrm{NO}\left(2.7 \times 10^{-12} \exp (350 / T) \mathrm{cm}^{3}\right.$ molec. $\left.^{-1} \mathrm{~s}^{-1}\right)$, as well as with $\mathrm{HO}_{2}\left(2.82 \times 10^{-13} \exp (1300 / T) \cdot[1-\right.$ $\exp (-0.231 n)] \mathrm{cm}^{3}$ molec. $^{-1} \mathrm{~s}^{-1}$, with $n$ the number of heavy atoms in the radical, excluding the peroxy moiety).

We also follow Wennberg et al. (2018) for estimating the nitrate yield in the reactions of organic peroxys with NO. The parameterization is based on the temperature- and pressuredependent expressions proposed by Carter and Atkinson (1989) and by Arey et al. (2001), modified to account for the recommendation by Teng et al. (2015) to relate this yield to the number $(n)$ of heavy atoms in the peroxy radical, excluding the peroxy moiety. The branching ratios of the nitrate pathway $\left(Y_{\text {nit }}\right)$ and oxy radical pathway $\left(Y_{\text {oxy }}\right)$ are given by

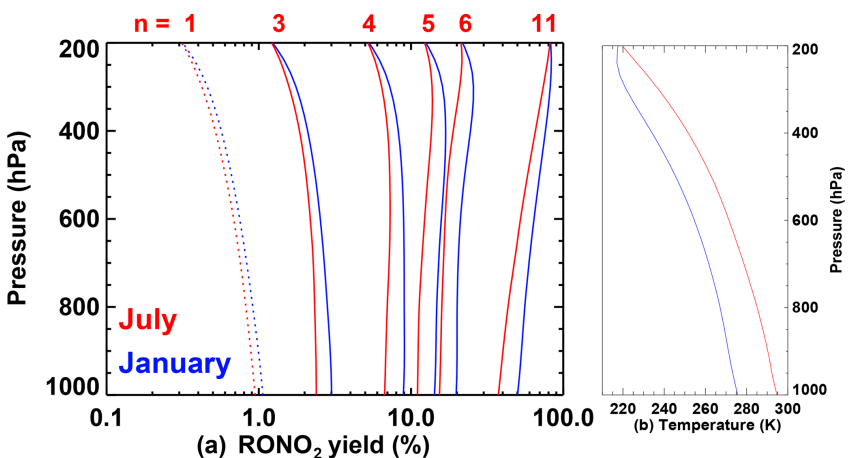

Figure 2. (a) Organic nitrate yield in the reaction of peroxy radicals with NO calculated following Wennberg et al. (2018) as a function of atmospheric pressure, using temperature profiles typical of January (in blue) and July (in red) at $40^{\circ} \mathrm{N}$ (zonal average of ECMWF analyses). The temperature profiles are shown in panel (b). $n$ is the number of heavy atoms in the peroxy radical. For $n=1$, the yield is calculated with $Z=1$ in Eq. (3).

$Y_{\text {nit }}(T, M, n, Z)=\frac{A(T, M, n)}{A(T, M, n)+Z}$,
$Y_{\text {oxy }}(T, M, n, Z)=1-Y_{\text {nit }}(T, M, n, Z)$,

with

$A(T, M, n)=\frac{k_{0}[M]}{1+k_{0}[M] / k_{\infty}} \cdot 0.41^{\left\{1+\left[\log _{10}\left(k_{0}[M] / k_{\infty}\right)\right]^{2}\right\}^{-1}}$,

$k_{0}=\alpha \cdot \mathrm{e}^{n}$

$k_{\infty}=0.43 \cdot(T / 298)^{-8}$,

where $\alpha=2 \times 10^{-22} \mathrm{~cm}^{3}$ molec. ${ }^{-1} . Z$ is a normalization term adjusted in order to match experimental determinations of the branching ratio, when available. In the absence of such a constraint, it is calculated (for $n>2$ ) using

$Z=A_{0}(n) \frac{1-\alpha_{0}}{\alpha_{0}}$,

with $A_{0}(n)=A\left(T=293 \mathrm{~K}, M=2.45 \times 10^{19}\right.$ molec. $\mathrm{cm}^{-3}$, $n)$ and

$\alpha_{0}=0.045 \cdot n-0.11$.

The nitrate yield is further modified according to molecular structure as recommended in Wennberg et al. (2018). The dependence of the yields on atmospheric pressure is shown in Fig. 2 for January and July at mid-latitudes. For small values of $n$ (especially $n=1), Y_{\text {nit }}$ decreases with altitude. For large values of $n$ (e.g. $n=11$ ), the yield increases with altitude due to the strong temperature dependence of the high-pressure limit (Eq. 7). 


\section{$2.7 \quad \mathrm{CH}_{3} \mathrm{O}_{2}+\mathrm{OH}$}

Methylperoxy radical $\left(\mathrm{CH}_{3} \mathrm{O}_{2}\right)$ was shown to react rapidly with $\mathrm{OH}$ (Bossolasco et al., 2014) although two more recent experimental studies inferred a lower rate constant (Yan et al., 2016; Assaf et al., 2016). The possible pathways include

$$
\begin{aligned}
\mathrm{CH}_{3} \mathrm{O}_{2}+\mathrm{OH} & \stackrel{\mathrm{a}}{\longrightarrow} \mathrm{CH}_{3} \mathrm{O}+\mathrm{HO}_{2} \\
& \stackrel{\mathrm{b}}{\longrightarrow} \mathrm{CH}_{3} \mathrm{OH}+\mathrm{O}_{2} \\
& \stackrel{\mathrm{c}}{\longrightarrow} \mathrm{CH}_{2} \mathrm{O}_{2}+\mathrm{H}_{2} \mathrm{O} \\
& \stackrel{\mathrm{d}}{\longrightarrow} \mathrm{CH}_{3} \mathrm{OOOH} .
\end{aligned}
$$

The stabilized trioxide $\left(\mathrm{CH}_{3} \mathrm{OOOH}\right)$ formed in channel $\mathrm{d}$ has several possible fates, among which reaction with $\mathrm{OH}$ and uptake by aqueous aerosols followed by decomposition into $\mathrm{CH}_{3} \mathrm{OH}+\mathrm{O}_{2}$ are expected to be the most important (Müller et al., 2016). An upper limit of $5 \%$ for the yield of Criegee radicals was also determined by Assaf et al. (2017), in agreement with the theoretical expectation that it should be negligible (Müller et al., 2016). A yield of $0.9 \pm 0.1$ for the methoxy $+\mathrm{HO}_{2}$ channel was determined experimentally at low pressure (67 hPa) (Assaf et al., 2018), in good agreement with the best theoretical estimate (0.92, range $0.77-0.97)$ determined in Müller et al. (2016) and used in our mechanism. It is also consistent with the methanol yield measurements reported recently by Caravan et al. (2018) at both low and high pressure $(0.06 \pm 0.02$ at 740 Torr). Those results imply, however, a methanol yield much lower than the value (0.23) used in our global model to reconcile its predictions with atmospheric methanol observations at remote locations (Müller et al., 2016). Note that at low pressure (as used in the experiments by Assaf et al., 2017 and Assaf et al., 2018), stabilization of the trioxide is negligible, given the quadratic dependence of the stabilization fraction $\left(f_{\text {stab }}\right)$ on atmospheric pressure (Müller et al., 2016),

$f_{\text {stab }}=f_{0} \cdot p^{2} \cdot(T / 298)^{-5}$,

where $p$ is atmospheric pressure (atm) and $T$ is temperature (K). In the lower troposphere, however, stabilization is significant, with a best theoretical estimate of $f_{0}=0.107$. Significant experimental evidence for this partial stabilization was found by Caravan et al. (2018) at 740 Torr (but not at low pressure).

The mechanism does not account for the possible reaction of $\mathrm{OH}$ with other peroxy radicals. As noted by Müller et al. (2016), its relevance for larger peroxys (such as those formed in the oxidation of biogenic VOCs) is expected to be lower than in the case of $\mathrm{CH}_{3} \mathrm{O}_{2}$. Furthermore, the fate of the stabilized trioxide formed at high yield (Müller et al., 2016; Assaf et al., 2018) in the reaction of large $\mathrm{RO}_{2}$ radicals with $\mathrm{OH}$ is so far unexplored.

\subsection{Notes to Table 2}

N1: Rate equal to $90 \%$ of evaluation (Burkholder et al., 2015 ) to account for isoprene-OH segregation (Pugh et al., 2011). See Sect. 2.1.1 for main products. The minor addition channels $(7 \%)$ include a hydroxyperoxy radical (ISOPEO2), as well as unsaturated carbonyls along with $\mathrm{HO}_{2}$. The unsaturated carbonyls are replaced by their major further oxidation products at high $\mathrm{NO}$ according to MCM (ACETO2 + $\mathrm{HCHO}+\mathrm{HO}_{2}+\mathrm{CO}_{2}$ ).

N2: See Sect. 2.2. The stabilized Criegee intermediate $\left(\mathrm{CH}_{2} \mathrm{OO}\right)$ is currently not a model compound; its production is replaced by the products of its main atmospheric sink, the reaction with the water dimer, namely $0.55 \mathrm{HMHP}+$ $0.4 \mathrm{HCHO}+0.4 \mathrm{H}_{2} \mathrm{O}_{2}+0.05 \mathrm{HC}(\mathrm{O}) \mathrm{OH}$ (Sheps et al., 2017).

N3: $Y^{\mathrm{nit}}(T, M, n, Z)$ denotes the nitrate yield, as defined in Sect. 2.6. $Z$ is adjusted to match laboratorybased estimates at room conditions $(\sim 298 \mathrm{~K}$ and $1 \mathrm{~atm})$ : $14 \%$ and $13 \%$ for the 1,2- and 4,3-isoprene hydroxyperoxys and $12 \%$ for the $\delta$-hydroxyperoxys (Wennberg et al., 2018). $Y^{\text {oxy }}(T, M, n, Z)$ (equal to $\left.1-Y^{\text {nit }}(T, M, n, Z)\right)$ is the oxy radical channel branching ratio. The reaction products account for the relative proportions of $\beta$ - and $\delta$ hydroxyperoxys (Sect. 2.1.3) as well as for the different organic nitrate yields in their reactions with NO.

N4: Bulk 1,6 H-shift reaction. See Sect. 2.1.1 for the rate and Sect. 2.1.2 for the products.

N5: See Sect. 2.1.2 for details.

N6: Addition channels (Wennberg et al., 2018). The product yields account for the small contribution of the $\delta$ hydroxyperoxy pathways. The minor $\delta$-IEPOX compounds are lumped with $\beta$-IEPOX. The non-IEPOX products observed by St. Clair et al. (2016) in presence of NO (HYAC, GLYALD, HPAC, $\mathrm{CH}_{3} \mathrm{CHO}$ ), as well as the dihydroxy dihydroperoxides (ISOP $(\mathrm{OOH})_{2}$ ), proposed as a potentially significant component of isoprene $\mathrm{SOA}$ in low- $\mathrm{NO}_{x}$ conditions (Liu et al., 2016), are assumed to have a negligible yield in most atmospheric conditions due to the proposed isomerization of the peroxy radical formed in the reaction (D' Ambro et al., 2017). The further chemistry of the dihydroxy hydroperoxy epoxide resulting from this isomerization, DHHEPOX, is not considered. Its saturation vapour pressure is estimated to be of the order of $3 \times 10^{-9}$ atm at $298 \mathrm{~K}$ using a group contribution method (Compernolle et al., 2011), i.e. 3 orders of magnitude lower than the estimated vapour pressure of $\beta$ IEPOX ( $\left.3 \times 10^{-6} \mathrm{~atm}\right)$. The Henry's law constant (HLC) of DHHEPOX estimated as described in Müller et al. (2018) is equal to $\sim 3 \times 10^{9} \mathrm{M} \mathrm{atm}^{-1}$ at $298 \mathrm{~K}$, almost 3 orders above the estimated value for IEPOX. DHHEPOX is, therefore, very probably more soluble and prone to loss by deposition or SOA formation than IEPOX, which has been shown to deposit very rapidly on vegetation (Nguyen et al., 2015b) and to be a prominent SOA precursor (Surratt et al., 2010). Furthermore, the products of the oxidation of DHHEPOX by OH (at a rate estimated at $\sim 2.1 \times 10^{-11}$ molec. $^{-1} \mathrm{~cm}^{3} \mathrm{~s}^{-1}$ ) are also 
expected to consist, for the most part, of highly oxygenated products prone to deposition and heterogeneous uptake.

N7: Abstraction of hydroperoxide-H $(75 \%)$ and of hydroxy- $\alpha$-H (25\%) (Wennberg et al., 2018). The latter leads to a radical proposed as undergoing epoxide formation (Wennberg et al., 2018); we neglect this very minor and uncertain pathway as the product was suggested to be due to an impurity (St. Clair et al., 2016). Addition of $\mathrm{O}_{2}$ to the radical forms $\mathrm{HO}_{2}+\mathrm{O}=\mathrm{CHC}\left(\mathrm{CH}_{3}\right)(\mathrm{OOH}) \mathrm{CH}=\mathrm{CH}_{2}$. The main fate of the unsaturated hydroperoxyaldehyde is photolysis to an enol, $\mathrm{HOCH}=\mathrm{C}\left(\mathrm{CH}_{3}\right) \mathrm{CH}=\mathrm{CH}_{2}(80 \%)$ or to $\mathrm{HCO}+\mathrm{OH}+\mathrm{MVK}(20 \%)$ (see Sect. 2.1.4). The enol reacts primarily by $\mathrm{OH}$ addition to the first carbon, followed by a $1,5 \mathrm{H}$-shift to $\mathrm{OH}+\mathrm{HC}(\mathrm{O}) \mathrm{OH}+\mathrm{MVK}$.

N8: Abstraction of hydroperoxide-H (60\%) and hydroxy$\alpha-\mathrm{H}$ (40\%), followed by similar reactions as for ISOPBOOH (see previous note). Hydroperoxy- $\alpha-\mathrm{H}$ abstraction is neglected.

N9: Assume fast reaction of MCM product with $\mathrm{OH}$, followed by fast reaction with $\mathrm{NO}$, neglecting side products.

N10: INBO2 is a mix of two peroxys (see Table 1). Assume $85 \%$ external and $15 \%$ internal $\mathrm{OH}$ addition to ISOPBNO3.

N11: The rates of the 1,5 and $1,6 \alpha$-hydroxy$\mathrm{H}$-shifts from the $\mathrm{C}_{1} \mathrm{HOCH}_{2}$ group in the radicals $\mathrm{HOCH}_{2} \mathrm{C}\left(\mathrm{CH}_{3}\right)\left(\mathrm{ONO}_{2}\right) \mathrm{CH}\left(\mathrm{O}_{2}\right) \mathrm{CH}_{2} \mathrm{OH}$ and $\mathrm{HOCH}_{2} \mathrm{C}\left(\mathrm{CH}_{3}\right)\left(\mathrm{ONO}_{2}\right) \mathrm{CH}(\mathrm{OH}) \mathrm{CH}_{2} \mathrm{O}_{2}$, respectively, suggested by Wennberg et al. (2018) are assumed equal to $0.02 \mathrm{~s}^{-1}$ at $298 \mathrm{~K}$ (instead of $0.05 \mathrm{~s}^{-1}$ in Wennberg et al., 2018) at the lower end of the range estimated by Møller et al. (2019) for $\alpha$-hydroxy-H-shifts, given the unfavourable $\mathrm{H}$-bonding between the peroxy group and the hydroxy-H of the other $\mathrm{C}_{4}$ or $\mathrm{C}_{3}$ alcohol group. The nitroxy hydroxy hydroperoxycarbonyls formed from the $\mathrm{H}$-shift are assumed to photolyse rapidly, releasing $\mathrm{HCO}, \mathrm{NO}_{2}$, and a hydroxyhydroperoxy carbonyl (here, $\mathrm{CH}_{3} \mathrm{C}(\mathrm{O}) \mathrm{CH}(\mathrm{OOH}) \mathrm{CH}_{2} \mathrm{OH}$ and $\mathrm{CH}_{3} \mathrm{C}(\mathrm{O}) \mathrm{CH}(\mathrm{OH}) \mathrm{CH}_{2} \mathrm{OOH}$, respectively, or $\left.\mathrm{MVKOOH}\right)$.

N12: Assume fast hydrolysis of the dinitrate in the aqueous aerosol phase, as it bears a tertiary nitrate group. The hydrolysis product (besides $\mathrm{HNO}_{3}$ ) is very soluble and can be assumed to remain in the particulate phase.

N13: The hydroperoxide bears a tertiary nitrate group and is assumed to undergo hydrolysis in the aerosol phase. The hydrolysis product (besides $\mathrm{HNO}_{3}$ ) is assumed to remain in the aerosol phase.

N14: As for INBO2 (see Note N11), the $1.5 \alpha$-hydroxy-Hshift in the peroxy $\mathrm{HOCH}_{2} \mathrm{C}\left(\mathrm{O}_{2}\right)\left(\mathrm{CH}_{3}\right) \mathrm{CH}\left(\mathrm{ONO}_{2}\right) \mathrm{CH}_{2} \mathrm{OH}$ is assumed to be 2.5 times slower compared to Wennberg et al. (2018). The nitroxy hydroxy hydroperoxycarbonyls formed from the $\mathrm{H}$-shift are assumed to photolyse rapidly, releasing $\mathrm{HCO}, \mathrm{NO}_{2}$ and a hydroxy hydroperoxycarbonyl $\left(\mathrm{HOCH}_{2} \mathrm{C}(\mathrm{OOH})\left(\mathrm{CH}_{3}\right) \mathrm{CHO}\right)$. The latter compound also photolyses very rapidly to $\mathrm{HCO}+\mathrm{OH}+\mathrm{HYAC}$.

N15: The hydroperoxyaldehyde $\left(\mathrm{O}=\mathrm{CHC}\left(\mathrm{CH}_{3}\right)(\mathrm{OOH}) \mathrm{CH}\left(\mathrm{ONO}_{2}\right) \mathrm{CH}_{2} \mathrm{OH}\right.$ or INDHPCHO in $\mathrm{MCM}$ ) formed in the reaction is assumed to photolyse rapidly to $\mathrm{HCO}+\mathrm{OH}+\mathrm{CH}_{3} \mathrm{C}(\mathrm{O}) \mathrm{CH}\left(\mathrm{ONO}_{2}\right) \mathrm{CH}_{2} \mathrm{OH}$.

N16: The trans and cis isomers are lumped, adopting the trans to cis ratio $(2: 1)$ of Bates et al. (2016). The epoxideretaining products are lumped into ICHE.

N17: Formyl-H abstraction from the carbonyl hydroxy epoxides (e.g. $\mathrm{HOCH}_{2} \mathrm{CHOC}\left(\mathrm{CH}_{3}\right) \mathrm{CHO}$ and isomers) primarily formed from IEPOX $+\mathrm{OH}$. The isomer distribution follows Wennberg et al. (2018). $\mathrm{H}$ abstraction is followed by concerted $\mathrm{CO}$ elimination and ring opening and $\mathrm{O}_{2}$ addition, leading to $\mathrm{CH}_{3} \mathrm{C}(\mathrm{O}) \mathrm{CH}\left(\mathrm{O}_{2}\right) \mathrm{CH}_{2} \mathrm{OH}$ (for the major isomer) and $\mathrm{OCHC}\left(\mathrm{O}_{2}\right)\left(\mathrm{CH}_{3}\right) \mathrm{CH}_{2} \mathrm{OH}$ (minor), which undergoes a 1,4 aldehyde-H-shift to $\mathrm{CO}+\mathrm{OH}+\mathrm{HYAC}$.

N18: Hydroxyl- $\alpha-\mathrm{H}$ abstraction from the carbonyl hydroxyepoxides (see previous note) at a rate taken to be equal to half the OH-reaction rate constant of $\beta$-IEPOX. It is followed by ring opening to give (for the main isomer) $\mathrm{OCHC}\left(\mathrm{CH}_{3}\right)\left(\mathrm{O}^{\circ}\right) \mathrm{CH}=\mathrm{CHOH}$, followed by 1,5 enol-H-shift and $\mathrm{O}_{2}$ addition to form $\mathrm{OCHC}\left(\mathrm{CH}_{3}\right)(\mathrm{OH}) \mathrm{CH}\left(\mathrm{O}_{2}\right) \mathrm{CHO}$. This is followed by a fast 1,5 aldehydic- $\mathrm{H}$-shift and (in large part) by $\mathrm{CO}$ elimination to give, after $\mathrm{O}_{2}$ addition, $\mathrm{CH}_{3} \mathrm{C}(\mathrm{O}) \mathrm{CH}(\mathrm{OOH}) \mathrm{CHO}+\mathrm{HO}_{2}$.

N19: The 1,4 H-shift in $\mathrm{HOCH}_{2} \mathrm{C}(\mathrm{OH})\left(\mathrm{CH}_{3}\right) \mathrm{CH}\left(\mathrm{O}_{2}\right) \mathrm{CHO}$ and its isomer is taken to be fast $\left(0.5 \mathrm{~s}^{-1}\right.$ at $\left.298 \mathrm{~K}\right)$, following Wennberg et al. (2018).

N20: The 1,5 H-shift in $\mathrm{HOCH}_{2} \mathrm{CH}(\mathrm{OH}) \mathrm{C}\left(\mathrm{CH}_{3}\right)\left(\mathrm{O}_{2}\right) \mathrm{CHO}$ forms $\mathrm{HO}_{2}+$ $\mathrm{O}=\mathrm{CHC}(\mathrm{OOH})\left(\mathrm{CH}_{3}\right) \mathrm{CH}(\mathrm{OH}) \mathrm{CHO}$, assumed to photolyse rapidly either to $\mathrm{CHO}+\mathrm{OH}+\mathrm{CH}_{3} \mathrm{C}(\mathrm{O}) \mathrm{CH}(\mathrm{OH}) \mathrm{CHO}$ (HOBA) or to $\mathrm{CHO}+\mathrm{HO}_{2}+\mathrm{OCHC}(\mathrm{OOH})\left(\mathrm{CH}_{3}\right) \mathrm{CHO}$ (HPDIAL).

N21: Oxy radical channel (65\%) (Wennberg et al., 2018).

N22: The hydroperoxide channel (35\%) forms $\mathrm{O}=\mathrm{CHC}(\mathrm{OOH})\left(\mathrm{CH}_{3}\right) \mathrm{CH}(\mathrm{OH}) \mathrm{CH}_{2} \mathrm{OH}$, assumed to photolyse very rapidly to $\mathrm{HCO}+\mathrm{OH}+\mathrm{CH}_{3} \mathrm{C}(\mathrm{O}) \mathrm{CH}(\mathrm{OH}) \mathrm{CH}_{2} \mathrm{OH}$.

N23: The 1,5 H-shift in $\mathrm{HOCH}_{2} \mathrm{C}(\mathrm{OH})\left(\mathrm{CH}_{3}\right) \mathrm{CH}\left(\mathrm{O}_{2}\right) \mathrm{CHO}$ forms $\mathrm{HO}_{2}+$ $\mathrm{O}=\mathrm{CHC}(\mathrm{OH})\left(\mathrm{CH}_{3}\right) \mathrm{CH}(\mathrm{OOH}) \mathrm{CHO}$ assumed to photolyse rapidly either to $\mathrm{CHO}+\mathrm{OH}+\mathrm{OCHC}\left(\mathrm{CH}_{3}\right)(\mathrm{OH}) \mathrm{CHO}$ or to $\mathrm{CHO}+\mathrm{HO}_{2}+\mathrm{CH}_{3} \mathrm{C}(\mathrm{O}) \mathrm{CH}(\mathrm{OOH}) \mathrm{CHO}$ (HPKETAL). The hydroxy dialdehyde is assumed to react exclusively with $\mathrm{OH}$, forming $\mathrm{CO}+\mathrm{MGLY}+\mathrm{HO}_{2}$.

N24: The hydroperoxide channel (35\%) forms $\mathrm{O}=\mathrm{CHCH}(\mathrm{OOH}) \mathrm{C}(\mathrm{OH})\left(\mathrm{CH}_{3}\right) \mathrm{CH}_{2} \mathrm{OH}$, assumed to photolyse very rapidly to $\mathrm{HCO}+\mathrm{OH}+$ $\mathrm{O}=\mathrm{CHC}(\mathrm{OH})\left(\mathrm{CH}_{3}\right) \mathrm{CH}_{2} \mathrm{OH}$.

N25: Neglect hydroperoxide channel, i.e. assume formation of oxy radical $+\mathrm{OH}$. Note that if the hydroperoxide is formed, it is expected to photolyse rapidly (Liu et al., 2018) in large part to the same products as the oxy radical pathway.

N26: Based on D'Ambro et al. (2017), the main OHaddition channel forms a hydroxyperoxy of which the main fate in low-NO regions should be reaction with $\mathrm{HO}_{2}$, followed by reaction of the hydroperoxide with $\mathrm{OH}$, form- 
ing $\mathrm{HOCH}_{2} \mathrm{CH}(\mathrm{OH}) \mathrm{C}\left(\mathrm{CH}_{3}\right)(\mathrm{OOH}) \mathrm{CHO}$ as the main product $(\mathrm{C} 75 \mathrm{OOH}$ in $\mathrm{MCM})$. Note that isomerization of the hydroperoxy also forms $\mathrm{C} 75 \mathrm{OOH}$ (along with $\mathrm{HO}_{2}$ ). $\mathrm{C} 57 \mathrm{OOH}$ is a $\alpha$-hydroperoxyaldehyde, assumed to photolyse rapidly (Liu et al., 2018) to $\mathrm{HCO}+\mathrm{OH}+\mathrm{CH}_{3} \mathrm{C}(\mathrm{O}) \mathrm{CH}(\mathrm{OH}) \mathrm{CH}_{2} \mathrm{OH}$, thus regenerating $\mathrm{OH}$ and $\mathrm{HO}_{2}$.

N27: The branching ratios are from Peeters and Müller (2010). The further mechanism mostly follows Wennberg et al. (2018); however, collisional deactivation of the radical $\left(\mathrm{OCHC}\left(\mathrm{CH}_{3}\right) \mathrm{C}^{\circ} \mathrm{CH}_{2}(\mathrm{OOH})\right)$ formed in the minor $\mathrm{OH}$ addition channel is neglected, since epoxide formation should be largely dominant, as for the radical formed by $\mathrm{OH}$ addition to ISOPOOH, for which epoxide formation constitutes ca. $90 \%$ of the sink. The unsaturated dialdehyde $\mathrm{O}=\mathrm{CHC}\left(\mathrm{CH}_{3}\right)=\mathrm{CHCH}(\mathrm{O})(\mathrm{MBED})$ undergoes very fast photolysis and is replaced by its oxidation products, as described in Sect. 2.1.5.

N28: Branching ratios from Peeters and Müller (2010); further, the mechanism from Wennberg et al. (2018), except for the collisional stabilization of the radical formed in the major addition channel, which is neglected (see previous note). As above, the unsaturated dialdehyde $\mathrm{O}=\mathrm{CHC}\left(\mathrm{CH}_{3}\right)=\mathrm{CHCH}(\mathrm{O})$ should photolyse rapidly to compounds replaced by their further reaction products. The hydroxy hydroperoxyaldehyde $\mathrm{HOOCH}_{2} \mathrm{C}\left(\mathrm{CH}_{3}\right)(\mathrm{OH}) \mathrm{CH}=\mathrm{O}$ should photolyse rapidly to (and is, therefore, replaced by) $\mathrm{HCO}+\mathrm{HO}_{2}+\mathrm{CH}_{3} \mathrm{C}(\mathrm{O}) \mathrm{CH}_{2} \mathrm{OOH}$ (HPACET).

N29: The peroxy radical $\left(\mathrm{CH}_{3} \mathrm{C}(\mathrm{O}) \mathrm{CH}(\mathrm{OH}) \mathrm{C}(\mathrm{O}) \mathrm{O}_{2}\right)$ formed in the reaction is replaced by its further oxidation products in presence of NO.

N30: $\mathrm{H}$ abstraction from $\mathrm{CH}$ group leads to $\mathrm{CH}_{3} \mathrm{C}(\mathrm{O}) \mathrm{C}(\mathrm{O}) \mathrm{CH}_{2} \mathrm{OOH}$, which can be assumed to photolyse very rapidly to $\mathrm{OH}+\mathrm{CH}_{3} \mathrm{C}(\mathrm{O}) \mathrm{O}_{2}+\mathrm{HCHO}+\mathrm{CO}$. $\mathrm{H}$ abstraction of the $\mathrm{CH}_{2}$ group yields $\mathrm{CH}_{3} \mathrm{C}(\mathrm{O}) \mathrm{CH}(\mathrm{OOH}) \mathrm{CHO}$ (HPKETAL).

N31: The acyl radical formed from $\mathrm{CH}_{3} \mathrm{C}(\mathrm{O}) \mathrm{CH}(\mathrm{OOH}) \mathrm{CHO}$ through aldehydic $\mathrm{H}$ abstraction can add $\mathrm{O}_{2}$ to form an acylperoxy radical that (upon reaction with $\mathrm{NO}$ ) leads to $\mathrm{CO}_{2}+\mathrm{OH}+\mathrm{MGLY}$. Note that the acyl radical can also decompose to $\mathrm{CO}+\mathrm{OH}+\mathrm{MGLY}$. Abstraction of the hydroperoxide $\mathrm{H}$ is followed by a 1,4 $\mathrm{H}$-shift of the peroxy radical $\mathrm{CH}_{3} \mathrm{C}(\mathrm{O}) \mathrm{CH}\left(\mathrm{O}_{2}\right) \mathrm{CHO}$ to the same acyl radical as above. $\mathrm{H}$ abstraction from the carbon bearing the $\mathrm{OOH}$ group ( $40 \%$ of reactivity) leads to $\mathrm{CH}_{3} \mathrm{C}(\mathrm{O}) \mathrm{C}(\mathrm{O}) \mathrm{CHO}$ assumed to photolyse rapidly to $\mathrm{CH}_{3} \mathrm{CO}+\mathrm{CO}+\mathrm{HCO}$.

N32: The acyl radical formed from $\mathrm{OCHC}\left(\mathrm{CH}_{3}\right)(\mathrm{OOH}) \mathrm{CHO}$ can add $\mathrm{O}_{2}$ to form an acylperoxy radical which (upon reaction with $\mathrm{NO}$ ) leads to $\mathrm{CO}_{2}+\mathrm{OH}+\mathrm{MGLY}$. Note that the acyl radical can also decompose to $\mathrm{CO}+\mathrm{OH}+$ MGLY.

N33: NISOPO2 is a mix of several radicals (Schwantes et al., 2015; Wennberg et al., 2018). The dinitrate formed in the reaction is ignored, as its further chemistry is unclear.
N34: See Sect. 2.3. A higher self-reaction rate was used by Schwantes et al. (2015) in their kinetic modelling, but there is suggestion that it might be overestimated (Schwantes et al., 2015).

N35: $\mathrm{H}$ abstraction from $\mathrm{HOOCH}_{2} \mathrm{CH}=\mathrm{C}\left(\mathrm{CH}_{3}\right) \mathrm{CH}_{2} \mathrm{ONO}_{2}$ and isomer.

N36: $\mathrm{OH}$ addition to $\mathrm{HOOCH}_{2} \mathrm{CH}=\mathrm{C}\left(\mathrm{CH}_{3}\right) \mathrm{CH}_{2} \mathrm{ONO}_{2}$ (for $84 \%$ ) and isomer $(16 \%)$. The mechanism follows Wennberg et al. (2018), except that (1) the 1,5 H-shift in the peroxy $\mathrm{O}_{2} \mathrm{NOCH}_{2} \mathrm{C}\left(\mathrm{O}_{2}\right)\left(\mathrm{CH}_{3}\right) \mathrm{CH}(\mathrm{OH}) \mathrm{CH}_{2} \mathrm{OH}$ (and isomer) formed in the reaction is neglected, as it should be slow due to stabilization by H-bonding between the peroxy and hydroxy groups; (2) epoxide formation (ca. $9 \%$ yield) is neglected; (3) the minor pathways in the bimolecular reactions of the hydroxyperoxy radicals, e.g. dinitrate formation in $\mathrm{RO}_{2}+\mathrm{NO}$ and dihydroperoxide formation in $\mathrm{RO}_{2}+\mathrm{HO}_{2}$, as well as the minor oxy decomposition channel proposed by Wennberg et al. (2018), are neglected since their yields are small and uncertain; (4) the peroxys are replaced by the products of their reactions with $\mathrm{NO}$ or $\mathrm{HO}_{2}$; and (5) the nitroxy hydroperoxy aldehyde $\mathrm{OCH}-\mathrm{C}\left(\mathrm{CH}_{3}\right)(\mathrm{OOH}) \mathrm{CH}_{2} \mathrm{ONO}_{2}$ is assumed to photolyse rapidly (Liu et al., 2018) to $\mathrm{CHO}+$ $\mathrm{OH}+\mathrm{CH}_{3} \mathrm{C}(\mathrm{O}) \mathrm{CH}_{2} \mathrm{ONO}_{2}$.

N37: The minor products $\mathrm{C} 3 \mathrm{CNO} 2$ and $\mathrm{C} 3 \mathrm{CPO} 2$ are replaced by assumed further oxidation product (NOA). The nitroxy hydroperoxyepoxide (IHPE) formed in the reaction (Schwantes et al., 2015) is neglected and the other yields are increased for carbon balance.

N38: $\quad \mathrm{H}$ abstraction from $\mathrm{CH}_{2}=\mathrm{CHC}\left(\mathrm{CH}_{3}\right)(\mathrm{OOH}) \mathrm{CH}_{2} \mathrm{ONO}_{2}$ and isomer.

N39: $\mathrm{OH}$ addition to $\mathrm{CH}_{2}=\mathrm{CHC}\left(\mathrm{CH}_{3}\right)(\mathrm{OOH}) \mathrm{CH}_{2} \mathrm{ONO}_{2}$ and isomer. The mechanism follows Wennberg et al. (2018), with simplifications similar to the case of the $\delta$-hydroperoxynitrates (see Note $\mathrm{N} 36$ ). The peroxy radical $\mathrm{O}_{2} \mathrm{NOCH}_{2} \mathrm{C}\left(\mathrm{CH}_{3}\right)(\mathrm{OOH}) \mathrm{CH}(\mathrm{OH}) \mathrm{CH}_{2} \mathrm{O}_{2}$ (INPHO $\beta$ in Schwantes et al., 2015) is assumed to react fast with $\mathrm{NO}$ or $\mathrm{NO}_{3}$, leading to $\mathrm{O}_{2} \mathrm{NOCH}_{2} \mathrm{C}\left(\mathrm{CH}_{3}\right)(\mathrm{OOH}) \mathrm{CHO}(\mathrm{C} 4 \mathrm{CPNA}$ in Schwantes et al., 2015) assumed to photolyse rapidly (Liu et al., 2018) to $\mathrm{CHO}+\mathrm{OH}+\mathrm{NOA}$.

N40: IHNE is a mix of two $\beta$ - and two $\delta$-nitroxy hydroxyepoxides. The mechanism follows Wennberg et al. (2018). The peroxy radicals $\mathrm{O}_{2} \mathrm{NOCH}_{2} \mathrm{C}(\mathrm{OH})\left(\mathrm{CH}_{3}\right) \mathrm{C}(\mathrm{O}) \mathrm{CH}_{2} \mathrm{O}_{2}$ and $\mathrm{HOCH}_{2} \mathrm{C}\left(\mathrm{O}_{2}\right)\left(\mathrm{CH}_{3}\right) \mathrm{CH}_{2} \mathrm{ONO}_{2}$ formed from the $\beta$-IHNE are replaced by the products of their reaction with $\mathrm{NO}$, neglecting dinitrate formation and minor oxy decomposition products. The radical $\mathrm{O}=\mathrm{C}^{\circ} \mathrm{CH}_{2} \mathrm{ONO}_{2}$ formed in these reactions adds $\mathrm{O}_{2}$, forming an acylperoxy radical replaced by its further reaction product in presence of $\mathrm{NO}$, i.e. $\mathrm{CO}_{2}+\mathrm{HCHO}+\mathrm{NO}_{2}$. The peroxy $\mathrm{O}_{2} \mathrm{NOC}(\mathrm{OH})\left(\mathrm{CH}_{3}\right) \mathrm{CH}\left(\mathrm{O}_{2}\right) \mathrm{CHO}$ undergoes a fast $1,4 \mathrm{H}$-shift outrunning bimolecular reactions, forming $\mathrm{CO}+\mathrm{OH}+\mathrm{O}_{2} \mathrm{NOCH}_{2} \mathrm{C}(\mathrm{OH})\left(\mathrm{CH}_{3}\right) \mathrm{CHO}$, which is assumed to photolyse rapidly to $\mathrm{NO}_{2}+\mathrm{HCHO}+\mathrm{MGLY}+\mathrm{HO}_{2}$ (Müller et al., 2014). The carbonyl nitroxyepoxides (ICNE in Wennberg et al., 2018) are assumed to react with $\mathrm{OH}$, 
following the Caltech reduced mechanism: ICNE $+\mathrm{OH} \rightarrow$ $2 \mathrm{CO}+0.35 \mathrm{NOA}+0.65 \mathrm{MGLY}+0.65 \mathrm{HO}_{2}+0.65 \mathrm{NO}_{2}$. The peroxys $\mathrm{O}_{2} \mathrm{NOCH}_{2} \mathrm{C}(\mathrm{OH})\left(\mathrm{CH}_{3}\right) \mathrm{CH}\left(\mathrm{O}_{2}\right) \mathrm{CHO}$ and $\mathrm{OCHC}\left(\mathrm{O}_{2}\right)\left(\mathrm{CH}_{3}\right) \mathrm{CH}(\mathrm{OH}) \mathrm{CH}_{2} \mathrm{ONO}_{2}$ formed from the $\delta$-IHNE undergo fast $\mathrm{H}$-shift reactions outrunning the bimolecular reactions, forming $\mathrm{CO}+\mathrm{OH}$ and either $\mathrm{O}_{2} \mathrm{NOC}(\mathrm{OH})\left(\mathrm{CH}_{3}\right) \mathrm{CH}\left(\mathrm{O}_{2}\right) \mathrm{CHO}$ (in the first case) or $\mathrm{CH}_{3} \mathrm{C}(\mathrm{O}) \mathrm{CH}(\mathrm{OH}) \mathrm{CH}_{2} \mathrm{ONO}_{2}$ (second case) (Wennberg et al., 2018).

N41: The $\mathrm{OH}$-reaction rate was measured by Xiong et al. (2016) for $\mathrm{OCHC}\left(\mathrm{CH}_{3}\right)=\mathrm{CHCH}_{2} \mathrm{ONO}_{2}$. The yields account for the $\mathrm{NC} 4 \mathrm{CHO}$ isomer distribution estimated by Schwantes et al. (2015). The $\mathrm{OH}$ reaction essentially follows Wennberg et al. (2018). Aldehyde $\mathrm{H}$ abstraction from $\mathrm{OCHCH}=\mathrm{C}\left(\mathrm{CH}_{3}\right) \mathrm{CH}_{2} \mathrm{ONO}_{2}$ by either $\mathrm{OH}$ or $\mathrm{NO}_{3}$ leads to an acylperoxy radical replaced here by its NO-reaction product according to $\mathrm{MCM}\left(\mathrm{CO}_{2}+\mathrm{CO}+\mathrm{HO}_{2}+\mathrm{NOA}\right)$. Note that alternative reaction pathways proposed by Wennberg et al. (2018) also lead eventually to $\mathrm{CO}+\mathrm{NOA}$. OH addition generates peroxy radicals undergoing fast isomerization (Schwantes et al., 2015), leading to the nitroxy hydroxyaldehyde $\mathrm{O}_{2} \mathrm{NOCH}_{2} \mathrm{C}(\mathrm{OH})\left(\mathrm{CH}_{3}\right) \mathrm{CHO}$ assumed to photolyse rapidly to $\mathrm{NO}_{2}+\mathrm{HCHO}+\mathrm{HO}_{2}+\mathrm{MGLY}$, the nitroxy hydroperoxy aldehyde $\mathrm{O}_{2} \mathrm{NOCH}_{2} \mathrm{C}\left(\mathrm{CH}_{3}\right)(\mathrm{OOH}) \mathrm{CHO}$ assumed to photolyse rapidly to $\mathrm{HCO}+\mathrm{OH}+\mathrm{NOA}$, and the nitroxy hydroperoxy ketone $\mathrm{CH}_{3} \mathrm{C}(\mathrm{O}) \mathrm{CH}(\mathrm{OOH}) \mathrm{CH}_{2} \mathrm{ONO}_{2}$ assumed to photolyse to $\mathrm{CH}_{3} \mathrm{CO}+\mathrm{OH}+\mathrm{OCHCH}_{2} \mathrm{ONO}_{2}$ (ETHLN).

N42: Abstraction of $\alpha$-hydroxy $\mathrm{H}$ in ISOPCNO3 $\left(\mathrm{HOCH}_{2} \mathrm{CH}=\mathrm{C}\left(\mathrm{CH}_{3}\right) \mathrm{CH}_{2} \mathrm{ONO}_{2}\right)$ and ISOPANO3 $\left(\mathrm{HOCH}_{2} \mathrm{C}\left(\mathrm{CH}_{3}\right)=\mathrm{CHCH}_{2} \mathrm{ONO}_{2}\right)$ in Wennberg et al. (2018), leading in part to photolabile hydroperoxy nitroxycarbonyls (e.g. $\mathrm{O}_{2} \mathrm{NOCH}_{2} \mathrm{C}(\mathrm{OOH})\left(\mathrm{CH}_{3}\right) \mathrm{CHO}$ ) assumed to photolyse rapidly (to either $\mathrm{HCO}+\mathrm{OH}+\mathrm{NOA}$ for ISOPCNO3, or $\mathrm{CH}_{3} \mathrm{CO}_{3}+\mathrm{OH}+\mathrm{ETHLN}$ for ISOPANO3).

N43: $\mathrm{OH}$ addition to ISOPCNO3 $\left(\mathrm{HOCH}_{2} \mathrm{CH}=\mathrm{C}\left(\mathrm{CH}_{3}\right) \mathrm{CH}_{2} \mathrm{ONO}_{2}\right.$ and ISOPANO3 $\left(\mathrm{HOCH}_{2} \mathrm{C}\left(\mathrm{CH}_{3}\right)=\mathrm{CHCH}_{2} \mathrm{ONO}_{2}\right)$. The mechanism follows Wennberg et al. (2018), except that two different dihydroxy nitroxyperoxy radicals are lumped into one radical (INCO2 or INAO2). In each case, only one of the two peroxy isomers undergoes an 1,5 H-shift. For simplicity, and since the $\mathrm{H}$-shift dominates largely the fate of the peroxy undergoing it, the bimolecular reactions are the reactions of the isomer that does not undergo the $\mathrm{H}$-shift.

N44: INCO2 includes two isomers, only one of which $\left(\mathrm{O}_{2} \mathrm{NOCH}_{2} \mathrm{C}\left(\mathrm{O}_{2}\right)\left(\mathrm{CH}_{3}\right) \mathrm{CH}(\mathrm{OH}) \mathrm{CH}_{2} \mathrm{OH}\right)$ undergoes an $1.5 \mathrm{H}$-shift. It leads to $\mathrm{HO}_{2}+$ $\mathrm{O}_{2} \mathrm{NOCH}_{2} \mathrm{C}(\mathrm{OOH})\left(\mathrm{CH}_{3}\right) \mathrm{CH}(\mathrm{OH}) \mathrm{CHO}$, assumed to be rapidly followed by fast photolysis (Liu et al., 2018) to $\mathrm{CHO}+\mathrm{HO}_{2}+\mathrm{O}_{2} \mathrm{NOCH}_{2} \mathrm{C}(\mathrm{OOH})\left(\mathrm{CH}_{3}\right) \mathrm{CHO}$, itself followed by photolysis to $\mathrm{CHO}+\mathrm{OH}+\mathrm{CH}_{3} \mathrm{C}(\mathrm{O}) \mathrm{CH}_{2} \mathrm{ONO}_{2}$ (NOA).

N45:

Wennberg et al. (2018). The hydroperoxide
$\mathrm{HOCH}_{2} \mathrm{CH}(\mathrm{OOH}) \mathrm{C}(\mathrm{OH})\left(\mathrm{CH}_{3}\right) \mathrm{CH}_{2} \mathrm{ONO}_{2}$ formed with a $43 \%$ yield is assumed to react with $\mathrm{OH}$, primarily by $\alpha$-hydroperoxide- $\mathrm{H}$ abstraction, forming $\mathrm{OH}+\mathrm{HOCH}_{2} \mathrm{C}(\mathrm{O}) \mathrm{C}(\mathrm{OH})\left(\mathrm{CH}_{3}\right) \mathrm{CH}_{2} \mathrm{ONO}_{2}$ (INCCO), and by abstraction of the terminal hydroperoxide hydrogen to regenerate $\mathrm{INCO} 2$.

N46: The dicarbonyl nitrate $\mathrm{O}_{2} \mathrm{NOCH}_{2} \mathrm{C}\left(\mathrm{CH}_{3}\right)(\mathrm{OH}) \mathrm{C}(\mathrm{O}) \mathrm{CHO}$ formed in the reaction is assumed to photolyse rapidly to $\mathrm{HCO}+\mathrm{O}_{2} \mathrm{NOCH}_{2} \mathrm{C}\left(\mathrm{CH}_{3}\right)(\mathrm{OH})-\mathrm{C}^{\circ}=\mathrm{O}$, which decomposes (in large part) into $\mathrm{CO}+\mathrm{HO}_{2}+\mathrm{O}_{2} \mathrm{NOCH}_{2} \mathrm{C}(\mathrm{O}) \mathrm{CH}_{3}$ (NOA).

N47: The mechanism follows the MCM. Among the three considered channels, formation of $\mathrm{O}_{2} \mathrm{NOCH}(\mathrm{CHO}) \mathrm{C}\left(\mathrm{CH}_{3}\right)(\mathrm{OH}) \mathrm{CH}_{2} \mathrm{ONO}_{2}+\mathrm{HO}_{2}$ is assumed to be followed by photolysis of the carbonyldinitrate to $\mathrm{NO}_{2}+\mathrm{GLY}+\mathrm{NOA}+\mathrm{HO}_{2}$ (Müller et al., 2014).

N48: INAO2 includes two peroxy isomers. The minor peroxy $\mathrm{HOCH}_{2} \mathrm{C}(\mathrm{OH})\left(\mathrm{CH}_{3}\right) \mathrm{CH}\left(\mathrm{O}_{2}\right) \mathrm{CH}_{2} \mathrm{ONO}_{2}$ can undergo an 1,5 $\alpha$-hydroxy-H-shift leading to $\mathrm{HO}_{2}+$ $\mathrm{OCHC}(\mathrm{OH})\left(\mathrm{CH}_{3}\right) \mathrm{CH}(\mathrm{OOH}) \mathrm{CH}_{2} \mathrm{ONO}_{2}$ (Wennberg et al., 2018), which is assumed to photolyse rapidly (Liu et al., $2018)$ to $\mathrm{CHO}+\mathrm{HO}_{2}+\mathrm{CH}_{3} \mathrm{C}(\mathrm{O}) \mathrm{CH}(\mathrm{OOH}) \mathrm{CH}_{2} \mathrm{ONO}_{2}$, itself followed by photolysis to $\mathrm{CH}_{3} \mathrm{CO}+\mathrm{OH}+\mathrm{OCHCH}_{2} \mathrm{ONO}_{2}$ (ETHLN).

N49: Adapted from Wennberg et al. (2018). The hydroperoxide product $(50 \%$ yield, $\left.\mathrm{HOCH}_{2} \mathrm{C}\left(\mathrm{CH}_{3}\right)(\mathrm{OOH}) \mathrm{CH}(\mathrm{OH}) \mathrm{CH}_{2} \mathrm{ONO}_{2}\right)$ is assumed to react with $\mathrm{OH}$, following the mechanism of the $\mathrm{MCM}$ and leading in part to $\mathrm{O}=\mathrm{CHC}\left(\mathrm{CH}_{3}\right)(\mathrm{OOH}) \mathrm{CH}(\mathrm{OH}) \mathrm{CH}_{2} \mathrm{ONO}_{2}$, which is assumed to photolyse rapidly to give $\mathrm{CHO}+\mathrm{OH}+\mathrm{CH}_{3} \mathrm{C}(\mathrm{O}) \mathrm{CH}(\mathrm{OH}) \mathrm{CH}_{2} \mathrm{ONO}_{2}$.

N50: The aldehyde-H-abstraction channel yields $\mathrm{HOCH}_{2} \mathrm{CH}=\mathrm{C}\left(\mathrm{CH}_{3}\right) \mathrm{C}(\mathrm{O}) \mathrm{O}_{2}$ or $\mathrm{HOCH}_{2} \mathrm{C}\left(\mathrm{CH}_{3}\right)=\mathrm{CHC}(\mathrm{O}) \mathrm{O}_{2}$ that should isomerize by $1,6 \mathrm{H}$-shifts of an $\alpha$-hydroxy- $\mathrm{H}$ to form the doubly resonance-stabilized radicals $Z$ $\mathrm{HOC}^{\circ} \mathrm{H}-\mathrm{CH}=\mathrm{C}\left(\mathrm{CH}_{3}\right)-\mathrm{C}(\mathrm{O}) \mathrm{OOH} \quad(\mathrm{Case} \quad \mathrm{I})$ or $Z$ $\mathrm{HOC}^{\circ} \mathrm{H}-\mathrm{C}\left(\mathrm{CH}_{3}\right)=\mathrm{CH}-\mathrm{C}(\mathrm{O}) \mathrm{OOH}$ (Case II). As for the similar $1,6 \mathrm{H}$-shifts in the initial $Z$ - $\delta$-OH-peroxys (see Sect. 2.1.2), the product radicals are expected to arise in both the $Z, Z^{\prime}$ and $Z, E^{\prime}$ forms, here assumed in a $50: 50$ ratio. The expected $\mathrm{O}_{2}$ addition-energy to these doubly resonance-stabilized radicals is as low as $15 \mathrm{kcal} \mathrm{mol}^{-1}$, such that $\mathrm{O}_{2}$ addition $\alpha$ to the $\mathrm{OH}$-group on $\mathrm{C}_{1}$ (or $\mathrm{C}_{4}$ ) is likely to result in $\mathrm{O}_{2}$ loss instead of concerted elimination of $\mathrm{HO}_{2}$, whereas $\mathrm{O}_{2}$ addition at the $\gamma$ position leads for $50 \%$ to $Z, Z^{\prime}$-peroxys that undergo fast $1,6 \mathrm{enol}-\mathrm{H}$-shifts facing barriers of only $10 \mathrm{kcal} \mathrm{mol}^{-1}$, similar to the $\mathrm{H}$-shifts leading to DIHPCARPs (Peeters et al., 2014). The product radical of these $\mathrm{H}$-shifts adds $\mathrm{O}_{2}$ to form DIHPCARP analogues that may readily isomerize by aldehyde-H-shift, promoted by $\mathrm{H}$-bonding. The resulting radicals are assumed to eliminate $\mathrm{CO}$ and $\mathrm{OH}$ to yield $\mathrm{OCHC}\left(\mathrm{CH}_{3}\right)(\mathrm{OOH}) \mathrm{C}(\mathrm{O}) \mathrm{OOH}$ or $\mathrm{CH}_{3} \mathrm{C}(\mathrm{O}) \mathrm{CH}(\mathrm{OOH}) \mathrm{C}(\mathrm{O}) \mathrm{OOH}$, which are ex- 
pected to photolyse rapidly (Liu et al., 2018) into $\mathrm{CO}+\mathrm{HO}_{2}+\mathrm{OH}+\mathrm{CH}_{3} \mathrm{C}(\mathrm{O}) \mathrm{C}(\mathrm{O}) \mathrm{OOH}$ or $\mathrm{CH}_{3} \mathrm{CO}_{3}+\mathrm{OH}+\mathrm{OCHC}(\mathrm{O}) \mathrm{OOH}$, respectively. Pyruvic peracid photolyses rapidly into $\mathrm{CH}_{3} \mathrm{CO}+\mathrm{CO}_{2}+\mathrm{OH}$, while its reaction with $\mathrm{OH}$ is very slow (Saunders et al., 2003). Peroxy glyoxylic acid (PGA) is considered explicitly. The $50 \% Z, E^{\prime}$-peroxys that also arise by $\gamma \mathrm{O}_{2}$ addition can react quasi-exclusively with $\mathrm{NO}$ and $\mathrm{HO}_{2}$, here assumed in a 50:50 ratio, to form mainly oxy radicals (e.g. $Z, E^{\prime}$ $\left.\mathrm{HOCH}=\mathrm{CHC}\left(\mathrm{CH}_{3}\right)\left(\mathrm{O}^{\circ}\right) \mathrm{C}(\mathrm{O}) \mathrm{OOH}\right)$ that quickly decompose into $\mathrm{CO}_{2}+\mathrm{OH}+$ either $\mathrm{CH}_{3} \mathrm{C}(\mathrm{O}) \mathrm{CH}=\mathrm{CH}_{2} \mathrm{OH}(\mathrm{HMVK})$ or $\mathrm{OCHC}\left(\mathrm{CH}_{3}\right)=\mathrm{CH}_{2} \mathrm{OH}(\mathrm{HMAC})$.

N51: OH-addition channel, with rates from Neeb (2000); Peeters et al. (2004). For OH addition $\beta$ to the formyl, we follow Wennberg et al. (2018), with product radicals IEPOXAO2 and IEPOXBO2 identical to those resulting from $\beta$-IEPOX $+\mathrm{OH}$. The peroxys from $\mathrm{OH}$ addition $\alpha$ to the formyl are unlikely to undergo 1,5 aldehyde- $\mathrm{H}$ shifts due to an unfavourable expected $\mathrm{H}$-bonding pattern but should rather react with $\mathrm{NO}$ or $\mathrm{HO}_{2}$ to yield mainly GLYALD + MGLY + $\mathrm{HO}_{2}$ for HALD1 or HYAC + GLY + $\mathrm{HO}_{2}$ for HALD2 (Peeters et al., 2004).

N52: Account for the fast isomerizations of the hydroxyperoxys resulting from $\mathrm{OH}$ addition to MACR (Crounse et al., 2012; Wennberg et al., 2018).

N53: Rate from MCM. The reactions occurs by $\alpha$-hydroxy-H abstraction, after which the three-ring opens to form the $10-15 \mathrm{kcal} \mathrm{mol}^{-1}$ more stable $\mathrm{HOCH}=\mathrm{C}\left(\mathrm{CH}_{3}\right)-\mathrm{C}(\mathrm{O}) \mathrm{O}^{\circ}$, the latter stabilized by acyloxy resonance. Direct elimination of $\mathrm{CO}_{2}$ as proposed in the MCM appears unlikely, since the $\mathrm{C}_{1}=\mathrm{C}_{2}--\mathrm{C}_{3}$ bond is $\sim 10 \mathrm{kcal} \mathrm{mol}^{-1}$ stronger than in $\mathrm{CH}_{3}--\mathrm{C}(\mathrm{O}) \mathrm{O}^{\circ}$ due to the neighbouring double bond. The most likely fate is a 1,5 enol-H-shift to $\mathrm{O}=\mathrm{CHC}^{\circ}\left(\mathrm{CH}_{3}\right) \mathrm{C}(=\mathrm{O}) \mathrm{OH}$ (with double "vinoxy" resonance-stabilization), exothermic for some $25-30 \mathrm{kcal} \mathrm{mol}^{-1}$ and almost barrierless. After adding $\mathrm{O}_{2}$, one can expect a 1,4 aldehyde- $\mathrm{H}$-shift followed by $\mathrm{CO}$ elimination (barrier $\sim 7 \mathrm{kcal} \mathrm{mol}^{-1}$ ) and $\mathrm{OH}$ loss to yield pyruvic acid. The latter is replaced by its photolysis products (Burkholder et al., 2015), i.e. $0.39 \mathrm{HO}_{2}+0.48 \mathrm{CH}_{3} \mathrm{CHO}+0.87 \mathrm{CO}_{2}+0.44 \mathrm{CH}_{3} \mathrm{C}(\mathrm{O}) \mathrm{O}_{2}+$ $0.08 \mathrm{CH}_{3} \mathrm{C}(\mathrm{O}) \mathrm{OH}+0.13 \mathrm{CO}+0.05 \mathrm{OH}$.

N54: See Note N2 regarding the stabilized Criegee intermediate $\left(\mathrm{CH}_{2} \mathrm{OO}\right)$. Pyruvic acid is replaced by its photolysis products (see previous Note).

N55: $\mathrm{MVKO} 2$ is a mix of $\mathrm{CH}_{3} \mathrm{C}(\mathrm{O}) \mathrm{CH}\left(\mathrm{O}_{2}\right) \mathrm{CH}_{2} \mathrm{OH}$ (72\%) and $\mathrm{CH}_{3} \mathrm{C}(\mathrm{O}) \mathrm{CH}(\mathrm{OH}) \mathrm{CH}_{2} \mathrm{O}_{2}(28 \%)$. The ratio is adjusted so that the glycolaldehyde yield in $\mathrm{MVKO} 2+\mathrm{NO}$ is $69 \%$ (Galloway et al., 2011), taking the nitrate yield (4\%) (Praske et al., 2015) into account.

N56: $\mathrm{MVKOOH}$ is a mix of $\mathrm{CH}_{3} \mathrm{C}(\mathrm{O}) \mathrm{CH}(\mathrm{OOH}) \mathrm{CH}_{2} \mathrm{OH}$ $(55 \%)$ and $\mathrm{CH}_{3} \mathrm{C}(\mathrm{O}) \mathrm{CH}(\mathrm{OH}) \mathrm{CH}_{2} \mathrm{OOH}(45 \%)$. The fractions account for the different hydroperoxide yields in the reaction of their respective peroxy radical precursors with $\mathrm{HO}_{2}$.
N57: Reaction rate taken equal to the average of the MCM and the structure-activity relationship (SAR) of Neeb (2000). Assume $50 \%$ formyl-H abstraction and $50 \%$ alcoholic- $\mathrm{H}$ abstraction. The former leads ultimately to hydroxyacetone + $\mathrm{NO}_{2}$ (in presence of $\mathrm{NO}$ ). The latter leads to a nitroxydialdehyde assumed to photolyse immediately into methylglyoxal, $\mathrm{NO}_{2}$, and $\mathrm{HCO}$.

N58: The reaction $\mathrm{MVKNO} 3+\mathrm{OH}$ is split into two reactions since MVKNO3 represents two isomers, $\mathrm{CH}_{3} \mathrm{C}(\mathrm{O}) \mathrm{CH}\left(\mathrm{ONO}_{2}\right) \mathrm{CH}_{2} \mathrm{OH}$ (for $80 \%$ ) and $\mathrm{CH}_{3} \mathrm{C}(\mathrm{O}) \mathrm{CH}(\mathrm{OH}) \mathrm{CH}_{2}\left(\mathrm{ONO}_{2}\right)$ (for $20 \%$ ). For the first, assume $50 \%$ alcoholic- $\mathrm{H}$ abstraction to $\mathrm{CH}_{3} \mathrm{C}(\mathrm{O}) \mathrm{CH}\left(\mathrm{ONO}_{2}\right) \mathrm{CHO}$, assumed to photolyse (for ca. $80 \%$ ) into $\mathrm{NO}_{2}+\mathrm{GLY}+\mathrm{CH}_{3} \mathrm{CO}$; the rest reacting with $\mathrm{OH}$ to eventually form MGLY $+\mathrm{HO}_{2}+\mathrm{CO}_{2}$ (in the presence of NO). For the second compound, ignore alcoholic-H abstraction.

N59: Assume fast reaction of the acylperoxy radical ( $84 \%$ of reactive flux) with NO. Assume fast photolysis of $\mathrm{CH}_{3} \mathrm{C}(\mathrm{O}) \mathrm{C}(\mathrm{O}) \mathrm{CHO}$ (16\% of flux) into $\mathrm{CH}_{3} \mathrm{CO}+\mathrm{CO}+$ HCO.

N60: Assume immediate reaction of product $\mathrm{OCHC}\left(\mathrm{CH}_{3}\right)(\mathrm{OH}) \mathrm{CHO}$ with $\mathrm{OH}$, forming $\mathrm{MGLY}+\mathrm{HO}_{2}+\mathrm{CO}_{2}$ upon reaction with $\mathrm{NO}$.

N61: The dominant $\mathrm{OH}$ addition, to $(\mathrm{HO})_{2} \mathrm{CHCH}\left(\mathrm{O}_{2}\right) \mathrm{C}(\mathrm{O}) \mathrm{CH}_{3}$, is followed by a $1,5 \mathrm{H}$-shift from an alcoholic-H to the peroxy group and decomposition (So et al., 2014). The minor addition channel forms $\mathrm{HOC}^{\circ} \mathrm{HCH}(\mathrm{OH}) \mathrm{C}(\mathrm{O}) \mathrm{CH}_{3}$, which reacts with $\mathrm{O}_{2}$ to $\mathrm{HO}_{2}+\mathrm{CH}_{3} \mathrm{C}(\mathrm{O}) \mathrm{CH}(\mathrm{OH}) \mathrm{CHO}$.

N62: The dominant $\mathrm{OH}$ addition $(3 \times$ $10^{-11}$ molec. $\left.^{-1} \mathrm{~cm}^{3} \mathrm{~s}^{-1}\right)$, to $\mathrm{O}=\mathrm{CHC}\left(\mathrm{CH}_{3}\right)\left(\mathrm{O}_{2}\right) \mathrm{CH}(\mathrm{OH})_{2}$, is followed by an $\mathrm{H}$-shift from either an alcoholic- $\mathrm{H}$ $(50 \%)$ or from the aldehyde- $\mathrm{H}(50 \%)$ to the peroxy group, leading to either $\mathrm{HC}(\mathrm{O}) \mathrm{OH}+\mathrm{OH}+\mathrm{MGLY}$ or $\mathrm{CO}+\mathrm{OH}+\mathrm{CH}_{3} \mathrm{C}(\mathrm{O}) \mathrm{CH}(\mathrm{OH})_{2}(\mathrm{DHA})$.

N63: Combines the minor addition channel $(1.2 \times$ $10^{-11}$ molec. $\left.^{-1} \mathrm{~cm}^{3} \mathrm{~s}^{-1}\right)$ and the aldehyde- $\mathrm{H}$ abstraction channel $\left(1.5 \times 10^{-11}\right.$ molec. $\left.^{-1} \mathrm{~cm}^{3} \mathrm{~s}^{-1}\right)$. The minor addition channel leads to $\mathrm{HO}_{2}+\mathrm{O}=\mathrm{CHC}\left(\mathrm{CH}_{3}\right)(\mathrm{OH}) \mathrm{CH}=\mathrm{O}$, which reacts primarily with $\mathrm{OH}$, leading to an acyl radical that can eliminate $\mathrm{CO}$ and give $\mathrm{MGLY}+\mathrm{HO}_{2}$ or form an acylperoxy radical that can undergo a shift of the aldehyde- $\mathrm{H}$ to the peroxy group. The resulting radical can either lose $\mathrm{CO}$, and upon reaction with $\mathrm{O}_{2}$, form $\mathrm{HO}_{2}+\mathrm{CO}+\mathrm{CH}_{3} \mathrm{C}(\mathrm{O}) \mathrm{C}(\mathrm{O}) \mathrm{OOH}$ (PPYR), or react with $\mathrm{O}_{2}$ and then with $\mathrm{NO}$ or $\mathrm{HO}_{2}$, forming $\mathrm{CO}_{2}+\mathrm{HO}_{2}+$ PPYR. The $\mathrm{H}$-abstraction channel leads to an acylperoxy radical, $\mathrm{O}=\mathrm{C}\left(\mathrm{O}_{2}\right) \mathrm{C}\left(\mathrm{CH}_{3}\right)=\mathrm{CHOH}$, which undergoes a enol 1,6 $\mathrm{H}$-shift followed by $\mathrm{O}_{2}$ addition to $\mathrm{O}=\mathrm{C}(\mathrm{OOH}) \mathrm{C}\left(\mathrm{O}_{2}\right)\left(\mathrm{CH}_{3}\right) \mathrm{CH}=\mathrm{O}$. The latter radical undergoes a 1,4 H-shift of the aldehyde- $\mathrm{H}$, leading to $\mathrm{CO}+\mathrm{OH}+$ PPYR. PPYR is assumed to photolyse rapidly to $\mathrm{CH}_{3} \mathrm{CO}+$ $\mathrm{CO}_{2}+\mathrm{OH}$ (Saunders et al., 2003).

N64: The nitrate yield is $1.3 \%$ at room conditions $(298 \mathrm{~K}$, $1 \mathrm{~atm})$. 
N65: Assume equal rates for the two addition channels. See Sect. 2.1.4.

N66: The reaction leads to pyruvic acid (along with $\mathrm{HO}_{2}$ ), assumed to photolyse very rapidly according to Burkholder et al. (2015).

N67: Yields calculated at room conditions. The acylperoxy radical resulting from $\mathrm{O}_{2}$ addition to the $\mathrm{HCOCO}$ radical (ca. $17 \%$ of the reactive flux) is replaced by the final reaction products in presence of $\mathrm{NO}$ and $\mathrm{O}_{2}$ (i.e. $\mathrm{CO}+\mathrm{HO}_{2}+\mathrm{CO}_{2}$ ).

N68: Contrary to MCM, consider aldehyde-H abstraction, leading in part to $\mathrm{CO}+\mathrm{OH}+\mathrm{HCHO}$ (for $25 \%$ ) and in part to $\mathrm{HOOCH}_{2} \mathrm{CO}_{3}(75 \%)$ which (upon reaction with $\mathrm{NO}$ ) leads to $\mathrm{CO}_{2}+\mathrm{OH}+\mathrm{HCHO}$.

N69: Reaction rate taken equal to the average of the MCM and the structure-activity relationship (SAR) of Neeb (2000). Products assume fast reaction of peroxy radical with NO.

N70: The minor channel $(8 \%$, formation of $\mathrm{CH}(\mathrm{OH})_{2} \mathrm{CH}_{2} \mathrm{O}_{2}$ ) proposed by So et al. (2014) is neglected.

N71: The methylnitrate yield adopted here is $2 \times 10^{-4}$ at $298 \mathrm{~K}$ and $1 \mathrm{~atm}$, or ca. $5 \times 10^{-5}$ in the lower stratosphere, at the lower end of the range $\left((5-10) \times 10^{-5}\right)$ estimated by Flocke et al. (1998) based on stratospheric $\mathrm{CH}_{3} \mathrm{ONO}_{2}$ observations.

N72: See Sect. 2.7 for details.

N73: The water dimer concentration (molec. $\mathrm{cm}^{-3}$ ) is calculated using

$[$ dimer $]=p \cdot K_{\mathrm{p}} \cdot\left[\mathrm{H}_{2} \mathrm{O}\right]^{2} /[M]$,

where $p$ is atmospheric pressure (atm), $\left[\mathrm{H}_{2} \mathrm{O}\right]$ and $\mathrm{M}$ are the water vapour and dry air number density (molec. $\mathrm{cm}^{-3}$ ), and $K_{\mathrm{p}}\left(\mathrm{atm}^{-1}\right)$ is approximated following Scribano et al. (2006):

$K_{\mathrm{p}}=4.7856 \times 10^{-4} \exp \left(1851.09 / T-5.10485 \times 10^{-3} T\right)$

N74: Rate reported by Wennberg et al. (2018). H abstraction from hydroperoxide group, followed by decomposition of the hydroxymethylperoxy radical, is slightly dominant (Allen et al., 2018). $\mathrm{H}$ abstraction from the carbon is followed by $\mathrm{OH}$ expulsion.

N75: The rate constant is for $\alpha$-pinene, although the compound APIN is a surrogate for all monoterpenes. For the products, see Sect. 2.4.

N76: The $26 \%$ yield is the assumed overall organic nitrate formation from monoterpenes (Rindelaub et al., 2015).

N77: Several carbonyl intermediates formed in the reaction are assumed to react rapidly with $\mathrm{OH}$. $\mathrm{CH}_{3} \mathrm{C}(\mathrm{OH})\left(\mathrm{CH}_{3}\right) \mathrm{C}(\mathrm{O}) \mathrm{O}_{2}$ is assumed to react with $\mathrm{NO}$, forming $\mathrm{CO}_{2}+\mathrm{CH}_{3} \mathrm{C}(\mathrm{O}) \mathrm{CH}_{3}+\mathrm{HO}_{2}$.

N78: The organic nitrate yield is $\sim 10 \%$ at room conditions (295 $\mathrm{K}$ and $1 \mathrm{~atm}$ ) (Chan et al., 2009). Whereas the major isomer peroxy radical leads to $\mathrm{CH}_{3} \mathrm{C}(\mathrm{O}) \mathrm{CH}_{3}+$ GLYALD $+\mathrm{HO}_{2}$ upon reaction with $\mathrm{NO}$, the other isomer leads to $\mathrm{HCHO}+\mathrm{HO}_{2}+\mathrm{CH}_{3} \mathrm{C}(\mathrm{OH})\left(\mathrm{CH}_{3}\right) \mathrm{CHO}$, which is here replaced by its $\mathrm{OH}$-reaction product in presence of $\mathrm{NO}$, namely $\mathrm{CO}_{2}+\mathrm{CH}_{3} \mathrm{C}(\mathrm{O}) \mathrm{CH}_{3}+\mathrm{HO}_{2}$. Note that the MCMv3.3.1 mechanism for MBO was recently validated by comparisons with chamber measurements, in particular regarding the production of radicals, acetone and formaldehyde (Novelli et al., 2018a), and that the peroxy radical isomerization reactions proposed by Knap et al. (2015) can be neglected due to their low rates and resulting impacts.

N79: The hydroperoxides formed in the reaction are replaced by the OH-reaction products in presence of NO.

N80: Average reactivity of the two isomer dihydroxynitrates. The products are replaced by their $\mathrm{OH}$-reaction products in presence of NO.

\subsection{Photodissociations}

The photolysis reactions are listed in Table 3. In many cases, the photolysis parameters are directly obtained from experimental studies or can be assumed identical to the parameters for other, similar compounds (e.g. the absorption cross sections of many organic hydroperoxides are assumed identical to those of $\mathrm{CH}_{3} \mathrm{OOH}$ ). For nitroxycarbonyls and for hydroperoxycarbonyls, however, analysis of the (scarce) available laboratory data indicates that the interaction between the two chromophores has a strong influence on the reaction mechanism and on the photodissociation parameters (Müller et al., 2014; Liu et al., 2018). The absorption cross sections for these classes (Fig. 3) are calculated based on available cross section data for structurally similar monofunctional compounds and on wavelength-dependent enhancement factors derived for nitroxycarbonyls (Müller et al., 2014) and for hydroperoxycarbonyls (Liu et al., 2018) based on available laboratory data.

\subsection{Uptake by aerosols}

The heterogeneous reactions on aerosols are listed in Table 4 with their associated reactive uptake coefficients. The rate $(\lambda)$ for the heterogeneous uptake of a chemical compound on aqueous aerosols is calculated using

$\lambda=\frac{A}{r_{n} / D_{\mathrm{g}}+4 /(\omega \cdot \gamma)}$,

where $A$ is the aerosol surface density $\left(\mathrm{cm}^{2} \mathrm{~cm}^{-3}\right), r_{n}$ is the number mean particle radius $(\mathrm{cm}), D_{\mathrm{g}}$ is the gas-phase diffusivity parameterized as described in Müller et al. (2008), $\omega$ is the mean molecular speed $\left(\mathrm{cm} \mathrm{s}^{-1}\right)$, and $\gamma$ the reactive uptake coefficient (Table 4). The aerosol surface density is calculated following Stavrakou et al. (2009b). Aqueous aerosols include inorganic (sulfate/ammonium/nitrate/water) and carbonaceous (OC and $\mathrm{BC}$ ) aerosols calculated by the model as described in Stavrakou et al. (2013) and sea salt aerosol from the MACC (Monitoring Atmospheric Composition and Climate) reanalysis (http://apps.ecmwf.int/datasets/ data/macc-reanalysis/levtype=sfc/, last access: 3 June 2019). 
(a) Hydroperoxycarbonyls and keto-enols

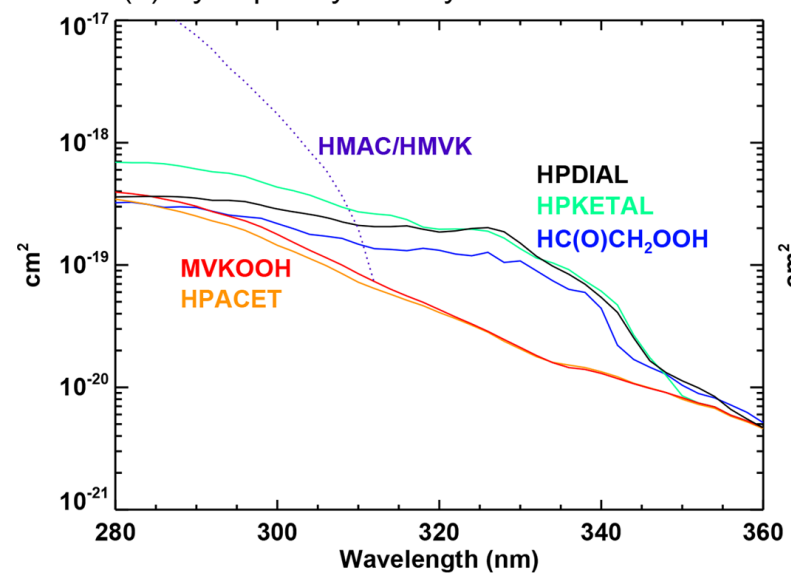

(b) Nitroxycarbonyls

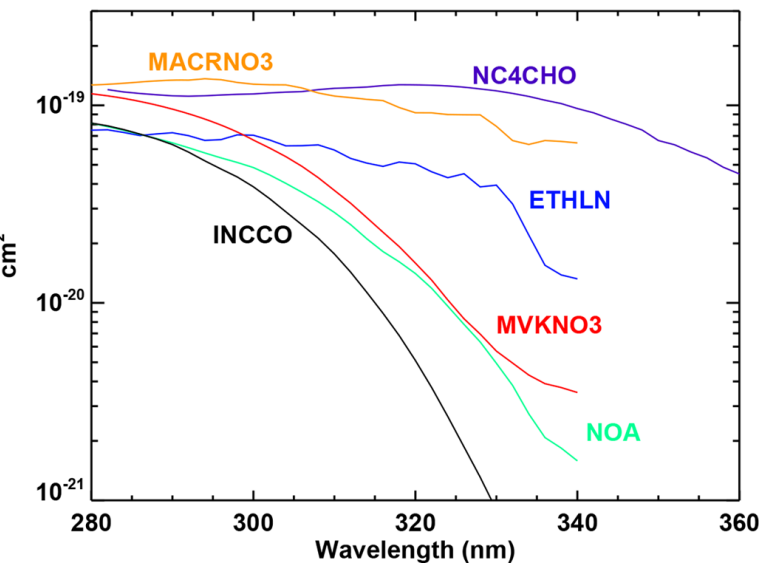

Figure 3. Absorption cross sections (in $\mathrm{cm}^{2}$ molec. ${ }^{-1}$ ) of (a) hydroperoxycarbonyls and keto-enols (HMAC and HMVK) and (b) nitroxycarbonyls. Species notation as in Table 1.

Table 4. Heterogeneous reactions on aqueous aerosols. $\gamma$ denotes the reactive uptake coefficient. References: 1, Liggio et al. (2005); 2, Marais et al. (2016); 3, Fisher et al. (2016); 4, Müller et al. (2016).

\begin{tabular}{lcc}
\hline Reaction & $\gamma$ & Ref. \\
\hline $\mathrm{GLY} \rightarrow \mathrm{GLY}($ aerosol $)$ & $2.9(-3)$ & 1 \\
$\mathrm{IEPOX} \rightarrow$ IEPOX (aerosol) & $4.2(-3)$ & $2^{\mathrm{a}}$ \\
$\mathrm{HMML} \rightarrow \mathrm{HMML}($ aerosol $)$ & $1.3(-4)$ & $2^{\mathrm{a}}$ \\
$\mathrm{ISOPBNO} 3 \rightarrow \mathrm{ISOPBOH}+\mathrm{HNO}_{3}$ & 0.03 & $\mathrm{~b}$ \\
$\mathrm{MACRNO} 3 \rightarrow \mathrm{MACROH}^{\mathrm{H}} \mathrm{HNO}_{3}$ & 0.03 & $\mathrm{~b}$ \\
$\mathrm{APINONO} 2 \rightarrow \mathrm{HNO}_{3}+$ product & 0.005 & 3 \\
$\mathrm{CH}_{3} \mathrm{OOOH} \rightarrow \mathrm{CH}_{3} \mathrm{OH}+\mathrm{O}_{2}$ & 0.1 & 4 \\
\hline
\end{tabular}

Notes: ${ }^{a}$ The dependence on aerosol pH (Marais et al., 2016; Stadtler et al., 2018 ) is ignored. ${ }^{b}$ See text (Sect. 2.10).

The heterogeneous uptake of alkyl nitrates by aqueous aerosols, followed by their hydrolysis has been suggested as a substantial organic nitrate sink and a large source of nitric acid in forested environments (Romer et al., 2016). Since tertiary nitrates were shown in the laboratory to undergo hydrolysis much faster than primary and secondary nitrates, we neglect the hydrolysis of non-tertiary nitrates while assuming fast hydrolysis of tertiary nitrates from isoprene. The reactive uptake coefficient $(\gamma)$ calculated by Marais et al. (2016) based on measured hydrolysis rates of a primary and a secondary hydroxynitrate from isoprene in neutral solution (Jacobs et al., 2014) is much too low $\left(1.3 \times 10^{-7}-5.2 \times 10^{-5}\right)$ to account for the loss observed during the Southern Oxidant and Aerosol Study (SOAS) campaign (Romer et al., 2016), due to the relatively low estimated Henry's law constant of isoprene hydroxynitrates. A much higher $\gamma(0.03)$ is assumed here for the major (tertiary) 1,2-hydroxynitrate from isoprene (ISOPBNO3), such that heterogeneous loss is its dominant fate in the troposphere, whereas the uptake of non-tertiary isoprene hydroxynitrates is neglected. Although crude, this assumption leads to a good model agreement against aircraft observations of isoprene hydroxynitrates over the southeastern US (see Sect. 4.2). Furthermore, the calculated average $\gamma$ for the sum of isoprene hydroxynitrates weighted by their respective abundances is $\sim 0.02$, consistent with the upper limit (0.02) inferred for the isoprene hydroxynitrate family by Wolfe et al. (2015) based on SOAS measurements. An uncertain but likely significant fraction of the monoterpene nitrates (represented in the mechanism by a unique lumped compound APINONO2) is assumed to be tertiary and undergoes hydrolysis (Browne et al., 2013, 2014) with $\gamma=0.005$ (Fisher et al., 2016). Other, minor tertiary nitrates generated in the mechanism (INB1OOH, INB2OOH, INB1NO3 in $\mathrm{MCM}$ ) are also assumed to undergo rapid uptake followed by hydrolysis in the aerosol, generating $\mathrm{HNO}_{3}$ and a usually very soluble and condensable co-product assumed to remain in the particulate phase. The saturation vapour pressures of those hydrolysis products (hydroperoxy triols and nitroxy triol) are calculated to be in the range $(4-40) \times 10^{-10}$ atm using the group contribution method of Compernolle et al. (2011), i.e. 3 orders of magnitude below the estimated vapour pressure of isoprene dihydroxy epoxide (IEPOX). The assumed rapid aerosol sink of the dinitrate INB1NO3 $\left(\mathrm{O}_{2} \mathrm{NOCH}\left(\mathrm{CH}_{2} \mathrm{OH}\right) \mathrm{C}\left(\mathrm{CH}_{3}\right)\left(\mathrm{ONO}_{2}\right) \mathrm{CH}_{2} \mathrm{OH}\right)$ generated in the oxidation of isoprene hydroxynitrates by $\mathrm{OH}$ has a potentially significant impact on total $\mathrm{RONO}_{2}$ levels, due to its expected, long chemical gas-phase lifetime, with an OH-rate constant of $\sim 2 \times 10^{-12}$ molec. $^{-1} \mathrm{~cm}^{3} \mathrm{~s}^{-1}$ (Saunders et al., 2003). However, a global model sensitivity simulation ignoring the aerosol sink of INB1NO3 and assuming similar gas-phase sink reactions as for the dinitrate IN$\mathrm{CNO} 3\left(\mathrm{HOCH}_{2} \mathrm{CH}\left(\mathrm{ONO}_{2}\right) \mathrm{C}\left(\mathrm{CH}_{3}\right)(\mathrm{OH}) \mathrm{CH}_{2} \mathrm{ONO}_{2}\right)$ shows that dinitrate hydrolysis depletes total $\mathrm{RONO}_{2}$ levels by only $\sim 3 \%$ globally, in spite of its strong impact on total dinitrate abundances (factor of 10). 
The hydrolysis of non-tertiary nitrates is slow compared to tertiary nitrates, and is, therefore, neglected here. Gasaerosol partitioning might occur, leading to possible loss by dry or wet aerosol deposition; this loss could be significant if repartitioning of particulate nitrates to the gas phase would be inhibited (Fisher et al., 2016). These effects are, however, very uncertain and are not considered here for simplicity.

\section{Box model comparison with other isoprene mechanisms}

\subsection{Description of simulations}

The isoprene mechanism is evaluated against the MCMv3.3.1 (obtained from http://mcm.leeds.ac.uk/MCM/, last access: 3 June 2019; Jenkin et al., 2015) and the Caltech reduced mechanism (version 4.3, obtained from https://doi.org/10.22002/D1.247 Wennberg et al., 2018). The Caltech mechanism is also available in its explicit ("full") version, which does not, however, include the further degradation of many terminal species down to $\mathrm{CO}_{2}$ and is, therefore, not appropriate for comparison. We perform $30 \mathrm{~h}$ simulations starting at 09:00 LT with $2 \mathrm{ppbv}$ isoprene. Temperature is set to $298 \mathrm{~K}$, and the $\mathrm{H}_{2} \mathrm{O}$ mixing ratio is $1 \%$. Two scenarios are considered: a high- $\mathrm{NO}_{x}$ scenario with 1 ppbv $\mathrm{NO}_{x}$ (also 40 ppbv $\mathrm{O}_{3}$ and $250 \mathrm{ppbv} \mathrm{CO}$ ) and a low- $\mathrm{NO}_{x}$ scenario with 100 pptv $\mathrm{NO}_{x}$ (with 20 ppbv $\mathrm{O}_{3}$ and $150 \mathrm{ppbv} \mathrm{CO}$ ). The photolysis rates are calculated for clear-sky conditions in mid-July at $30^{\circ} \mathrm{N}$, with $300 \mathrm{DU}$ ozone and an albedo of 0.05 using the Tropospheric Ultraviolet and Visible (TUV) photolysis model of Madronich (1993). For computational efficiency, the photolysis rates are parameterized as a function of solar zenith angle using MCM-type expressions (Saunders et al., 2003),

$J=l \cdot(\cos \chi)^{m} \cdot \exp (-n / \cos \chi)$,

where the parameters $l, m$, and $n$ are obtained from TUV calculations at three zenith angles $\left(0,30\right.$ and $\left.60^{\circ}\right)$. For convenience, the numbering of the photodissociations is the same as in the MCM, except for those (e.g. hydroperoxycarbonyls) for which the MCM falls back on simpler, monofunctional model compounds. Since Wennberg et al. (2018) does not provide specific recommendations for the calculation of photolysis rates, we use our own expressions in their mechanism. The Caltech mechanism files do include noontime photolysis rate estimates, but their derivation is unclear, and their use in the intercomparison would lead to large discrepancies with both MCM and MAGRITTE, obscuring the interpretation of differences. To further facilitate this interpretation, the same inorganic chemistry and the same rates of the major reactions of $\mathrm{CH}_{3} \mathrm{O}_{2}$ and $\mathrm{CH}_{3} \mathrm{CO}_{3}$ (with $\mathrm{NO}, \mathrm{HO}_{2}$, and $\mathrm{NO}_{2}$ ) as well as of PAN-like compounds are adopted in the three mechanisms. Heterogeneous uptake on aerosols is also included, calculated assuming an aerosol surface density of
$5 \times 10^{7} \mathrm{~cm}^{2} \mathrm{~cm}^{-3}$ with uptake coefficients as in Table 4. All rate coefficient expressions are available at the MAGRITTE mechanism repository (https://doi.org/10.18758/71021042).

\subsection{Comparison results for $\mathrm{HO}_{\mathrm{x}}$}

The temporal evolution of key compounds concentrations calculated with the three mechanisms using the Kinetic PreProcessor (KPP) package (Damian et al., 2002) are displayed in Fig. 4 (for high $\mathrm{NO}_{x}$ ) and Fig. 5 (low $\mathrm{NO}_{x}$ ). The initial isoprene is more rapidly consumed at high $\mathrm{NO}_{x}(<2 \mathrm{~h})$ than at low- $\mathrm{NO}_{x}(\sim 5 \mathrm{~h})$ due to higher $\mathrm{OH}$ levels $\left(\sim 10^{7}\right.$ vs. $\sim 2 \times 10^{6}$ molec. $\left.\mathrm{cm}^{-3}\right)$. There is generally a much better level of agreement between the mechanisms at high $\mathrm{NO}_{x}$ compared to low $\mathrm{NO}_{x}$. The Caltech mechanism leads to the highest $\mathrm{OH}$ levels. At low $\mathrm{NO}_{x}$, the Caltech-based average $[\mathrm{OH}]$ during the first $4 \mathrm{~h}$ of the numerical experiment is higher by a factor of 1.25 and 1.32 than with the MCM and MAGRITTE mechanisms, respectively. The Caltechbased model also predicts higher $\mathrm{HO}_{2}$ (by a factor of $\sim 1.1$ ), $\mathrm{CH}_{3} \mathrm{O}_{2}(\sim 1.3)$, and especially $\mathrm{CH}_{3} \mathrm{CO}_{3}(\sim 1.4)$. The differences between the three mechanisms do not exceed a few percent at high $\mathrm{NO}_{x}$. There are several causes for the large differences at low $\mathrm{NO}_{x}$.

The first reason is that the Caltech mechanism includes a higher direct $\mathrm{OH}$ yield (1.5) in the bulk 1,6-isomerization of isoprene peroxy radicals. This production is the result of the high assumed yield of DIHPCARP (0.6) in this reaction and of the high direct (1) and secondary (1.5) yield of $\mathrm{OH}$ radical resulting from the degradation of DIHPCARPs. Furthermore, the $\beta$-HPALDs also formed in the 1,6-isomerization of isoprene peroxys are mainly lost by photolysis, leading to additional $\mathrm{HO}_{x}$ production. As a sensitivity test, the model was run with the MAGRITTE mechanism modified by replacing the bulk 1,6 H-shift reaction of isoprene peroxys by its representation in the Caltech mechanism. This change alone increases $\mathrm{OH}$ concentrations by about $15 \%$ compared to the standard MAGRITTE simulation, and also reduces the discrepancies for $\mathrm{HO}_{2}, \mathrm{CH}_{3} \mathrm{O}_{2}$ and $\mathrm{CH}_{3} \mathrm{CO}_{3}$.

A second reason for lower $\mathrm{HO}_{x}$ levels lies in the yield of $\mathrm{HO}_{x}$ and other radicals in the photolysis of several major hydroperoxycarbonyls (e.g. HPAC, HPACET and HPKETAL). This yield is much lower in our mechanism, as it accounts for the major enol-forming channel (Liu et al., 2018), which does not produce any radical. Those reactions generate one $\mathrm{OH}$ and either one $\mathrm{HO}_{2}$ or one $\mathrm{CH}_{3} \mathrm{CO}_{3}$ radical in the Caltech mechanism, which assumes either scission of the $\mathrm{C}-\mathrm{C}$ bond followed by $\mathrm{OH}$ expulsion or (equivalently) direct $\mathrm{OH}$ release followed by splitting off of either formyl or acetyl radical. A second sensitivity calculation with the MAGRITTE mechanism, modified by assuming that the photolysis of those hydroperoxycarbonyls proceeds as in the Caltech mechanism, further increases $\mathrm{OH}$ by almost $10 \%$ in the first hours. Even larger increases are calculated $(\sim 20 \%)$ for $\mathrm{CH}_{3} \mathrm{O}_{2}$ and $\mathrm{CH}_{3} \mathrm{CO}_{3}$. 

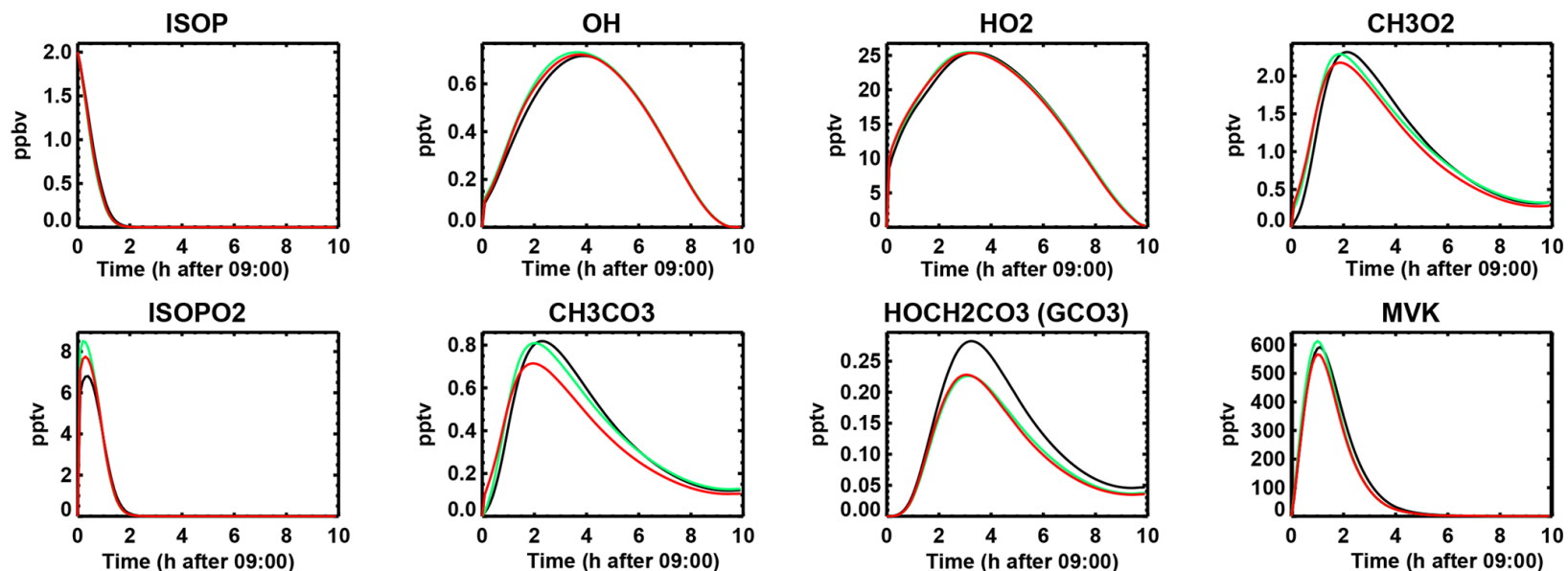

MACR

HALD
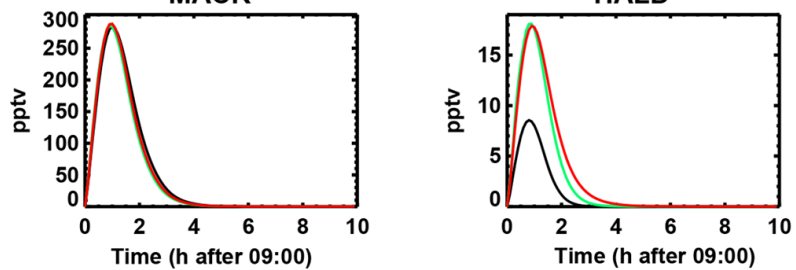

HPALD

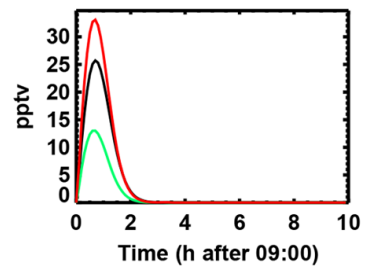

ISOPOOH

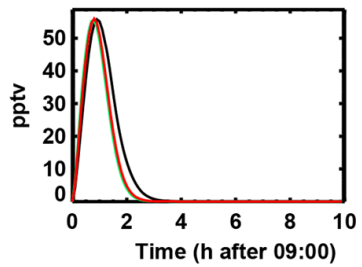

ISOPN
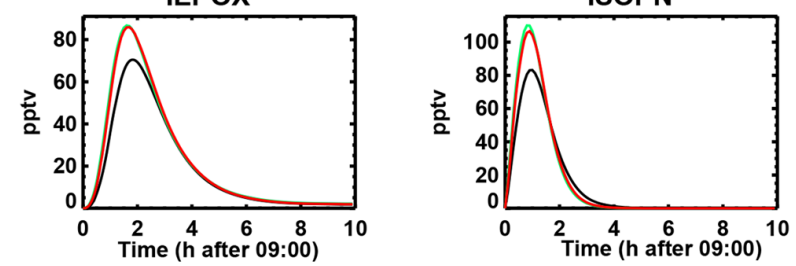

HMAC+HMVK

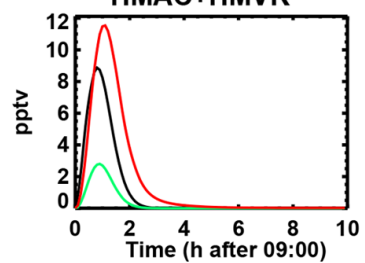

HPACET

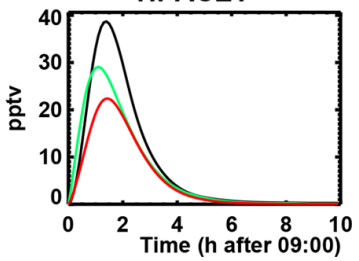

GLYALD
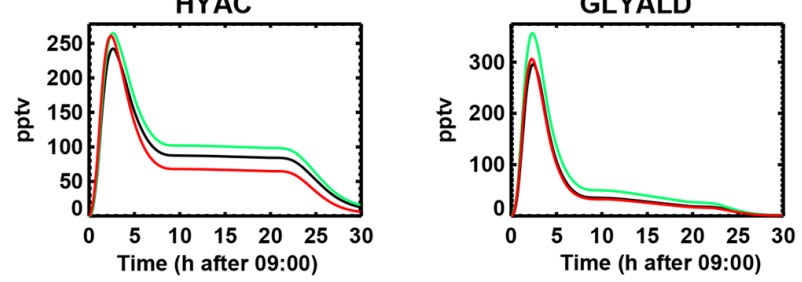

$\mathrm{HCHO}$

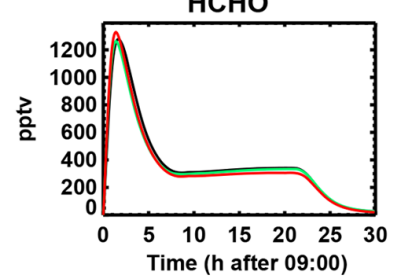

RONO2

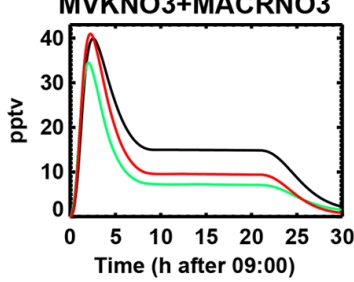

RO2NO2
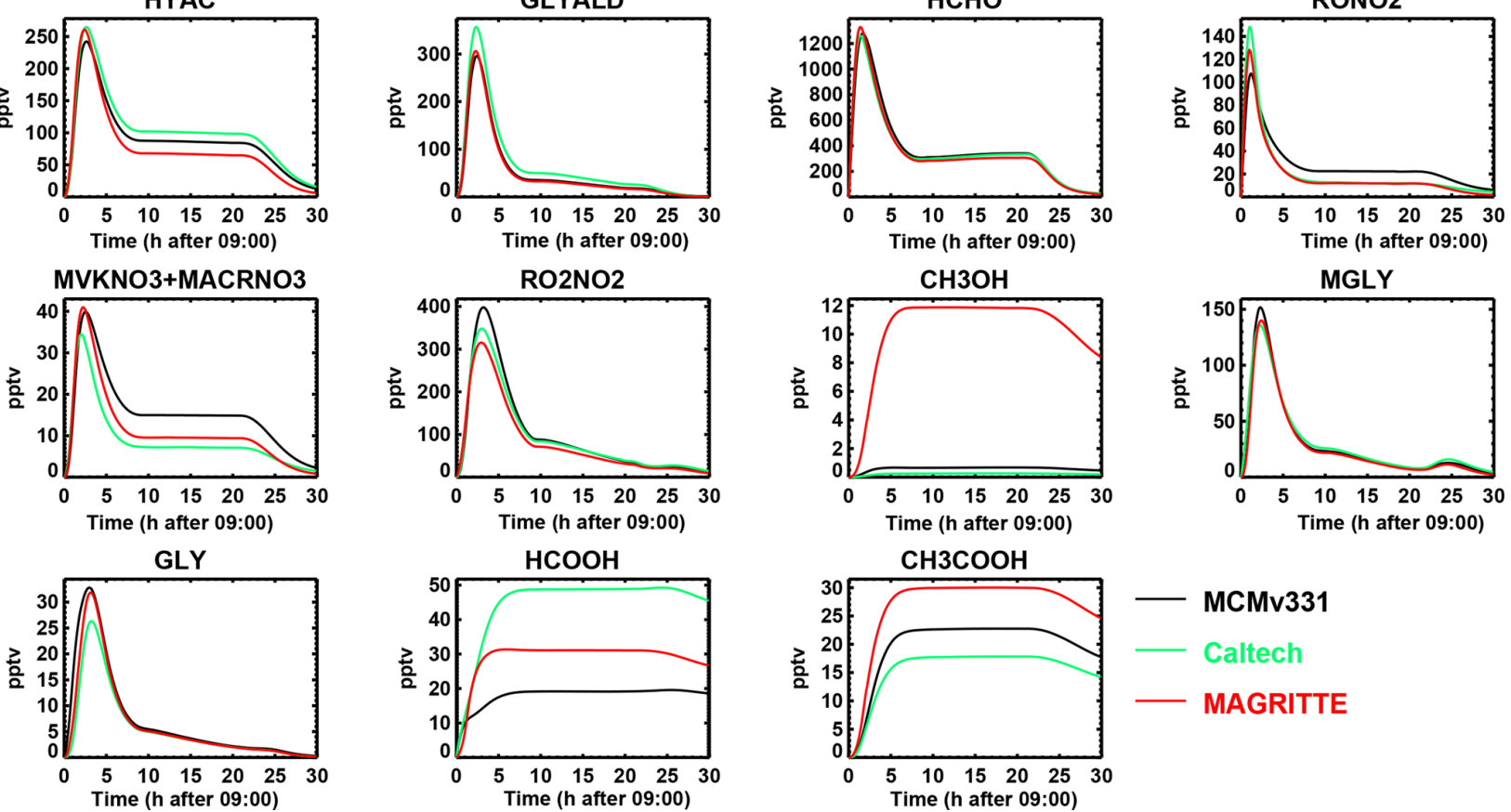

MCMv331

Caltech

MAGRITTE

Figure 4. Box-model-calculated mixing ratios of key compounds at $1 \mathrm{ppbv} \mathrm{NO}_{x}$. MCM results in black, Caltech mechanism in green, this work in red. ISOPN is the sum of isoprene hydroxynitrates; RONO2 is the sum of organic nitrates; RO2NO2 is the sum of PANs. 

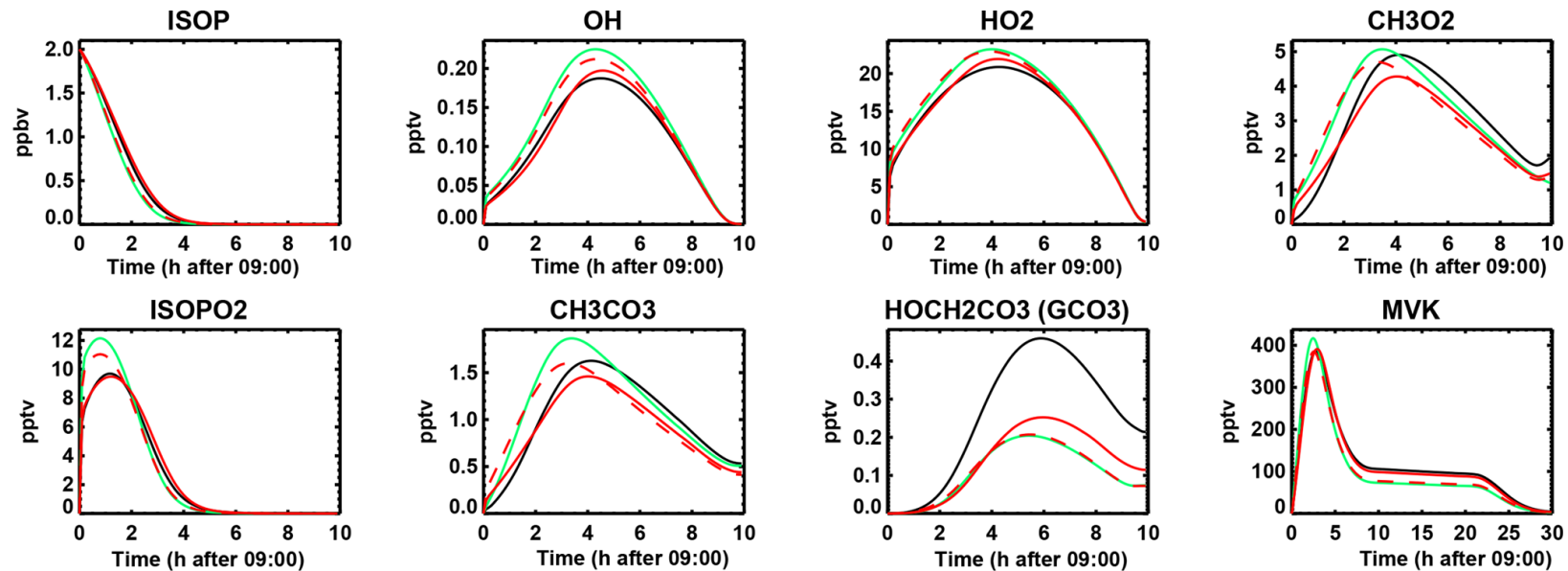

MACR

HALD

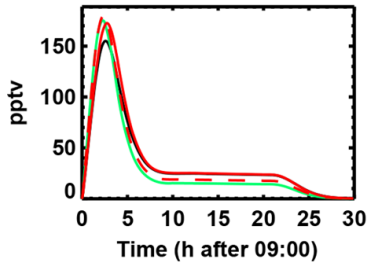

HPALD
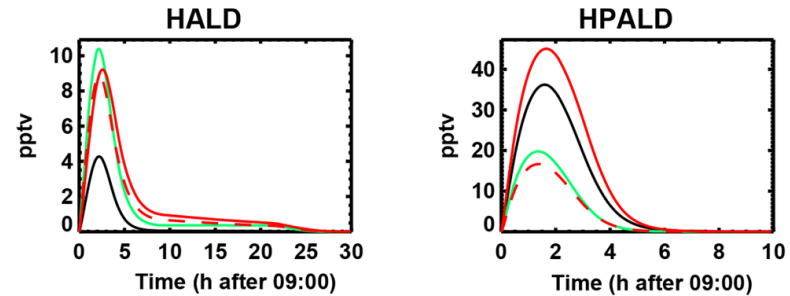

ISOPOOH

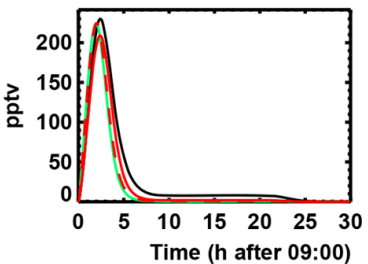

HMAC+HMVK

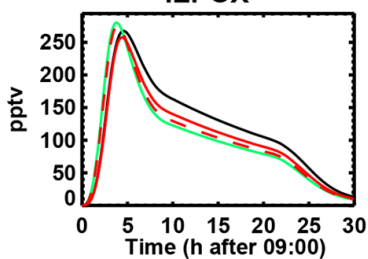

ISOPN
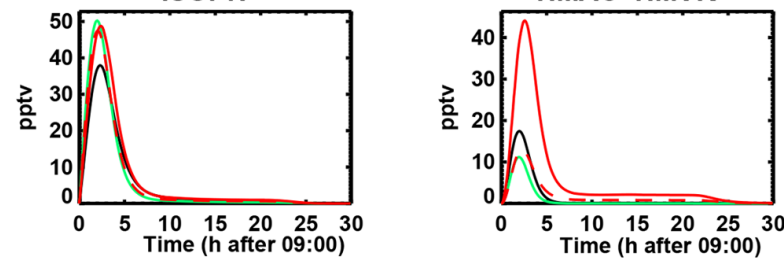

HPACET

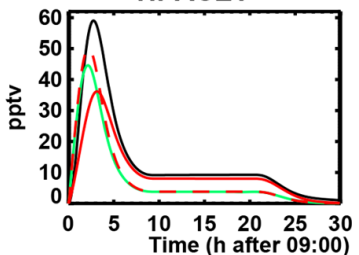

GLYALD
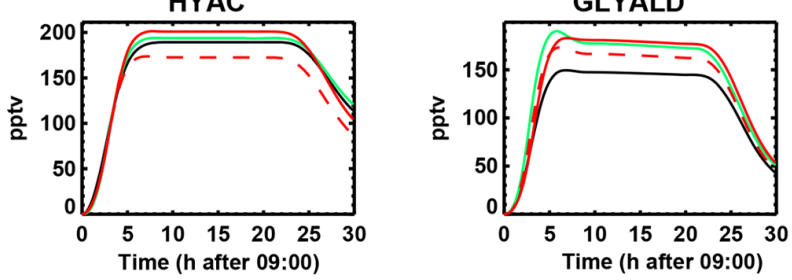

$\mathrm{HCHO}$

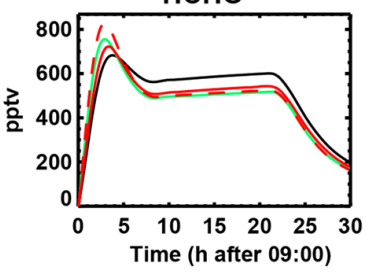

RONO2

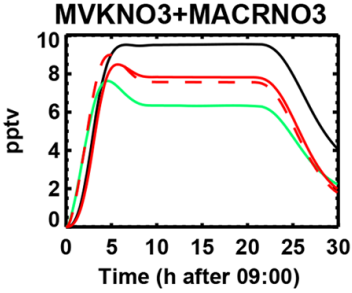

RO2NO2

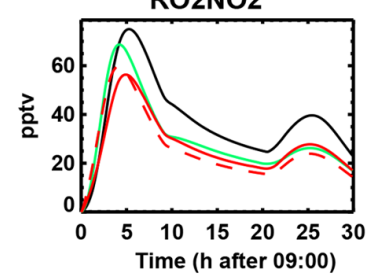

$\mathrm{CH} 3 \mathrm{OH}$
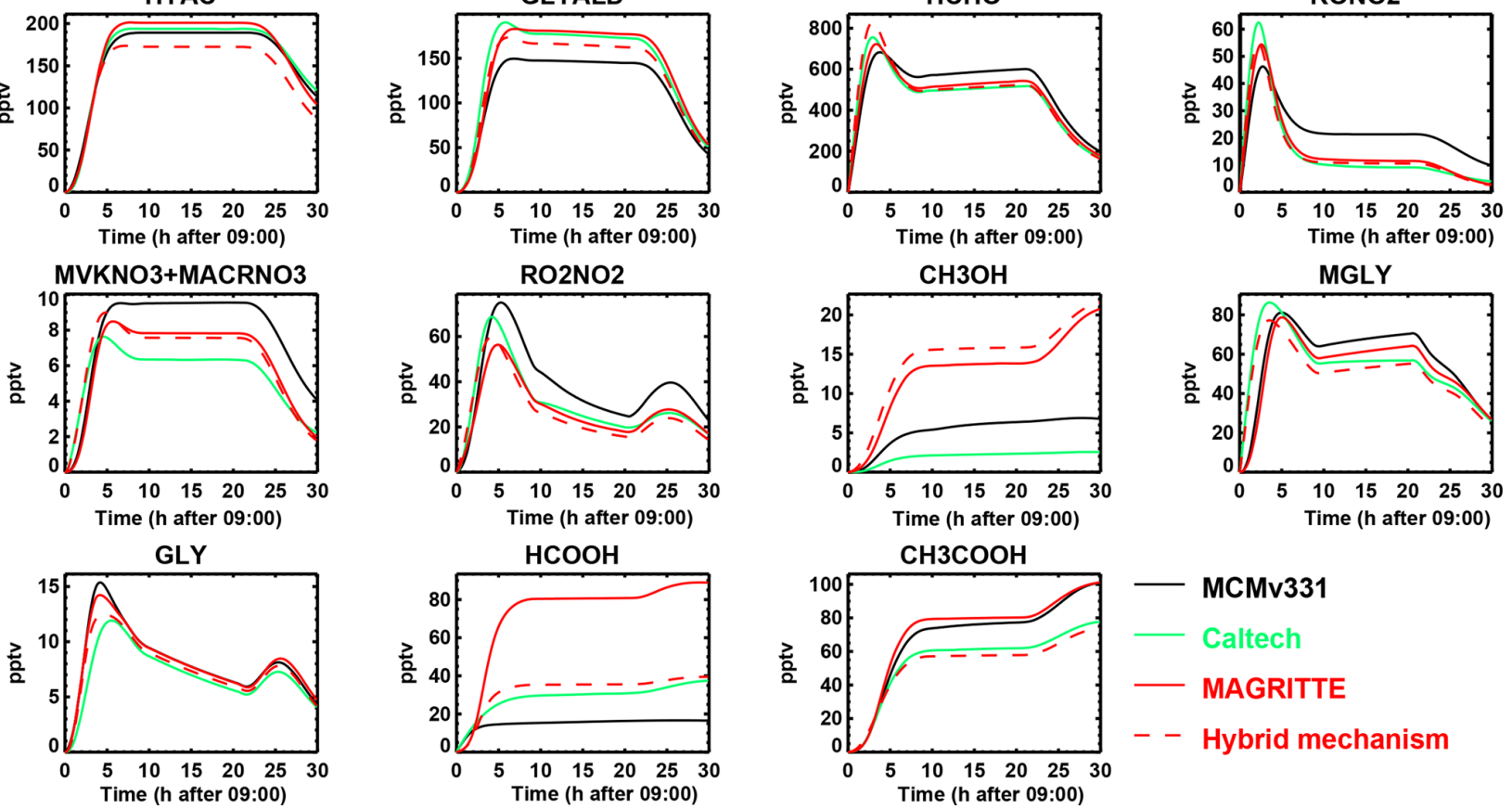

MCMv331

Caltech

MAGRITTE

- - Hybrid mechanism

Figure 5. As Fig. 4, for $100 \mathrm{ppt} \mathrm{NO}_{x}$. The dashed red line corresponds to a simulation using the MAGRITTE mechanism with the Caltech representation of the isoprene peroxy 1,6 H-shift and of the hydroperoxycarbonyl photolysis reactions. 
A lesser but significant factor also contributing to the differences includes the higher bulk 1,6-isomerization yield in the reduced Caltech mechanism, in large part due to the neglect of the minor $\mathrm{OH}$-addition pathways to the central carbons of isoprene, which represent $7 \%$ of the total ISOP $+\mathrm{OH}$ reaction flux in our mechanism.

The results of a sensitivity calculation using the MAGRITTE mechanism modified by adopting the Caltech reduced mechanism representation of (1) isoprene peroxy 1,6 H-shift yield and products and (2) hydroperoxycarbonyl photolysis reactions are shown in Fig. 5 ("Hybrid mechanism", dashed red lines). The residual differences between Caltech and the modified MAGRITTE mechanisms are very small (a few percent) for $\mathrm{HO}_{x}, \mathrm{CH}_{3} \mathrm{O}_{2}$, and $\mathrm{CH}_{3} \mathrm{CO}_{3}$.

\subsection{Comparison results for isoprene products}

The three mechanisms agree well for the main isoprene oxidation products (e.g. MVK, MACR, HCHO) when accounting for differences in OH levels and in the HPALD yield in the bulk 1,6-isomerization of isoprene peroxys $(0.25,0.5$, and 0.75 in the Caltech, MCM, and MAGRITTE mechanisms). The lower yield of primary hydroxynitrates (ISOPN) in ISOPO2+NO reactions in the MCM $(10 \%$, vs. $\sim 13 \%$ following Wennberg et al., 2018) explains the lower MCM ISOPN and total organic nitrate $\left(\mathrm{RONO}_{2}\right)$ concentrations during the first hours. Note that higher ISOPN and $\mathrm{RONO}_{2}$ levels (by a factor of $\sim 1.2$ ) are calculated when the aerosol sink of tertiary nitrates is not considered.

In spite of the similar ISOPN concentrations in the three simulations, the calculated $\mathrm{RONO}_{2}$ levels decrease more rapidly after the initial peak in the Caltech simulation than in the MAGRITTE and especially in the MCM simulation (Fig. 5). This is partly explained by differences in $\mathrm{OH}$, as seen from the lower discrepancy in $\mathrm{RONO}_{2}$ found between the Caltech and hybrid mechanism simulations that realize very similar $\mathrm{OH}$ levels. An additional cause of difference in $\mathrm{RONO}_{2}$ levels is the $1,5 \mathrm{H}$-shift in dihydroxy nitroxyperoxy radicals (INBO2 and INDO2) formed from the $\mathrm{OH}$ oxidation of isoprene hydroxynitrates. This H-shift forms hydroperoxynitroxy carbonyls assumed to photolyse very rapidly, releasing $\mathrm{NO}_{2}$ and, therefore, removing $\mathrm{RONO}_{2}$. It is the dominant sink of those peroxys in the Caltech simulation, while it is neglected in the MCM and assumed to proceed at a slower rate $\left(0.02 \mathrm{~s}^{-1}\right)$ in our mechanism, due to the influence of $\mathrm{H}$ bonding (see Notes N11 and N14). This also explains the higher abundance of the carbonylhydroxynitrates (MVKNO3 and MACRNO3) in the MCM and MAGRITTE simulations (Fig. 5), as those are partly formed from the bimolecular reactions of the peroxys INBO2 and INDO2.

Dinitrates make up only a very small contribution to total $\mathrm{RONO}_{2}$ levels in the simulations $\left(<0.5 \%\right.$ at low $\mathrm{NO}_{x}$, $<3 \%$ at high $\mathrm{NO}_{x}$ ). The dinitrates formed from ISOP+OH are indeed mostly tertiary and, therefore, assumed to hydrolyse rapidly to $\mathrm{HNO}_{3}$ and an alcohol. When the aerosol sink of those nitrates is neglected, their contribution to total $\mathrm{RONO}_{2}$ becomes substantial (13 pptv out of $52 \mathrm{pptv}$ at low $\mathrm{NO}_{x}$ ) in the MCM simulation but remains low in the Caltech simulation $(<2 \mathrm{pptv})$. This large difference stems mostly from lower dinitrate yield in the reactions of dihydroxy nitroxyperoxy radicals with NO in the Caltech mechanism, due to the strong reduction of the yield due to the nitrate group. Moreover, the MCM neglects the photolysis of the dinitrates, which represents about one-third of their total (non aerosol-related) sink according to our estimation. Both the aerosol reactions and the dinitrate yield are acknowledged as very uncertain, however, and the overall impact of dinitrates could be larger than assumed in our mechanism.

The total peroxynitrate (RO2NO2), methylglyoxal, and glyoxal concentrations calculated in the three simulations are in reasonable agreement. The differences in $\mathrm{RO} 2 \mathrm{NO} 2$ level are partly related to differences in yield of the $\mathrm{HOCH}_{2} \mathrm{C}(\mathrm{O}) \mathrm{O}_{2}$ radical $(\mathrm{GCO} 3)$ in the photolysis of $\mathrm{CH}_{3} \mathrm{C}(\mathrm{O}) \mathrm{C}(\mathrm{O}) \mathrm{CH}_{2} \mathrm{OH}$, equal to 1 in the MCM, 0.5 in our mechanism and 0 in the Caltech mechanism (see Note $t$ in Sect. 2.9).

The production of methanol, however, is much larger with MAGRITTE than with the MCM (factor of 3) and with the Caltech mechanism (factor of 8). A large part of this difference is due to the $\mathrm{CH}_{3} \mathrm{O}_{2}+\mathrm{OH}$ reaction (Sect. 2.7), which accounts for about half the $\mathrm{CH}_{3} \mathrm{OH}$ production at low $\mathrm{NO}_{x}$ and even more at high $\mathrm{NO}_{x}$. In addition, the rate of the $\mathrm{CH}_{3} \mathrm{O}_{2}+$ $\mathrm{RO}_{2}$ reactions has a unique value for all $\mathrm{RO}_{2}$ compounds $\left(3.5 \times 10^{-13}\right.$ molec. $^{-1} \mathrm{~cm}^{3} \mathrm{~s}^{-1}$ at $\left.298 \mathrm{~K}\right)$ in the MCM, much lower than in the Caltech and MAGRITTE mechanism for isoprene hydroxyperoxys $\left(2 \times 10^{-12} \mathrm{molec}^{-1} \mathrm{~cm}^{3}\right)$. Finally, although the full Caltech mechanism includes $\mathrm{CH}_{3} \mathrm{OH}$ formation in the reaction of, e.g. ISOPDO2 (4,3-ISOPOO) with $\mathrm{CH}_{3} \mathrm{O}_{2}$, this production is neglected in the reduced Caltech mechanism, explaining the very low Caltech-calculated methanol levels in Figs. 4-5.

Very large differences are also found for formic acid. In the first hour of the experiment, MAGRITTE predicts lower formation rates due to lower direct $\mathrm{HCOOH}$ formation from the ozonolysis of isoprene: in particular, the primary $\mathrm{HCOOH}$ yield is only about $3 \%$ in MAGRITTE, about 6 times less than in both the MCM and Caltech mechanism (at $1 \% \mathrm{H}_{2} \mathrm{O}$ mixing ratio). $\mathrm{HMHP}\left(\mathrm{HOCH}_{2} \mathrm{OOH}\right)$ not being formed in the MCM, the overall $\mathrm{HCOOH}$ production from alkene ozonolysis (both direct and indirect through HMHP oxidation) is slightly higher in MAGRITTE than in MCM, whereas it is about twice as high in the Caltech mechanism. At later times, the formation of formic acid due to the reactions of enols (VA, HMAC and HMVK) with $\mathrm{OH}$ becomes a larger source than the ozonolysis of isoprene and its degradation products according to MAGRITTE, especially at low $\mathrm{NO}_{x}$. The Caltech mechanism includes an additional $\mathrm{HCOOH}$ production pathway through the oxidation of secondary isoprene nitrates (e.g. $\left.\mathrm{CH}_{3} \mathrm{C}(\mathrm{O}) \mathrm{CH}\left(\mathrm{ONO}_{2}\right) \mathrm{CH}_{2} \mathrm{OH}\right)$ by $\mathrm{OH}$, which becomes significant at high $\mathrm{NO}_{x}$. This mechanism proposed by Paulot 
et al. (2009b) involves abstraction of an $\alpha$-hydroxy- $\mathrm{H}$, followed by $\mathrm{O}_{2}$ addition and by a rearrangement leading to $\mathrm{NO}_{3}+\mathrm{HCOOH}+\mathrm{MGLY}$, instead of the expected fast dissociation of the $\alpha$-hydroxyperoxy radical into $\mathrm{HO}_{2}$ and a dicarbonyl. This mechanism is ignored in our mechanism, as it is highly complex and likely faces a much higher barrier than the fast $\mathrm{HO}_{2}$ expulsion (at $\sim 1000 \mathrm{~s}^{-1}$, Hermans et al., 2005).

Finally, the production of acetic acid is relatively similar in the three mechanisms. The slightly lower acetic acid production in the Caltech run is primarily due to a lower $\mathrm{CH}_{3} \mathrm{C}(\mathrm{O}) \mathrm{OH}$ yield in the $\mathrm{CH}_{3} \mathrm{C}(\mathrm{O}) \mathrm{O}_{3}+\mathrm{HO}_{2}$ reaction (0.13 vs. 0.16 in MCM and MAGRITTE) and to the neglect of $\mathrm{CH}_{3} \mathrm{C}(\mathrm{O}) \mathrm{OH}$ formation through reactions of isoprene peroxys with $\mathrm{CH}_{3} \mathrm{CO}_{3}$. It is partly compensated for by higher $\mathrm{CH}_{3} \mathrm{CO}_{3}$ levels in the Caltech simulation, especially at low $\mathrm{NO}_{x}$. The MAGRITTE mechanism includes an additional acetic acid source through the $\mathrm{OH}$ oxidation of $\mathrm{CH}_{2}=\mathrm{C}\left(\mathrm{CH}_{3}\right) \mathrm{OH}$ (MVA) generated from the photolysis of hydroperoxyacetone HPACET. This source accounts for $\sim 28 \%$ and $38 \%$ of the total $\mathrm{CH}_{3} \mathrm{C}(\mathrm{O}) \mathrm{OH}$ source at high and low $\mathrm{NO}_{x}$, respectively.

\section{Regional and global modelling}

\subsection{Model description and simulations}

The MAGRITTE v1.1 model calculates the distribution of 182 chemical compounds, among which 141 species undergo transport processes (advection, deep convection, and turbulent diffusion) in the model. MAGRITTE can be run either globally at $2^{\circ}$ (latitude) $\times 2.5^{\circ}$ (longitude) resolution, or regionally at $0.5^{\circ} \times 0.5^{\circ}$ resolution. The lateral boundary conditions of the regional model are provided by the global model. In the vertical, the model uses a hybrid ( $\sigma$-pressure) coordinate, with 40 levels between the Earth's surface and the lower stratosphere ( $44 \mathrm{hPa}$ level). The meteorological fields are provided by ECMWF ERA-Interim analyses (Dee et al., 2011). Most model parameterizations, including the transport scheme and the chemical mechanism for anthropogenic and biomass burning VOCs are inherited from the IMAGES model (Muller and Brasseur, 1995; Stavrakou et al., 2009a, b, 2015; Bauwens et al., 2016). The deposition scheme is described in a companion paper (Müller et al., 2018).

The model uses anthropogenic emissions of $\mathrm{CO}, \mathrm{NO}_{x}$, $\mathrm{OC}, \mathrm{BC}$, and $\mathrm{SO}_{2}$ from the HTAPv2 dataset for year 2010 (Janssens-Maenhout et al., 2015). Following Travis et al. (2016), the anthropogenic $\mathrm{NO}_{x}$ emissions over the US are first scaled down to match the US total $\left(3.5 \mathrm{Tg} \mathrm{N} \mathrm{yr}^{-1}\right)$ for the year 2013 reported by the National Emission Inventory (NEI), and the US $\mathrm{NO}_{x}$ emissions due to industry and transport are further reduced by $60 \%$ to match observed aircraft $\mathrm{NO}_{x}$ concentrations and nitric acid deposition data, consistent with the recommendation of Anderson et al. (2014). Anthropogenic NMVOC emissions are provided by the EDGARv4.3.2 inventory (Huang et al., 2017) for the year 2012. The global annual anthropogenic NMVOC source is $154 \mathrm{Tg}$ NMVOC ( $118 \mathrm{Tg} \mathrm{C})$. Biomass burning emissions (78 Tg NMVOC or $45 \mathrm{Tg} \mathrm{C}$ in 2013) are obtained from the Global Fire Emission Database version 4 (GFED4s) (van der Werf et al., 2017) and are vertically distributed according to Sofiev et al. (2013).

Isoprene, monoterpene, and $\mathrm{MBO}$ fluxes (366, 91.5, and $0.93 \mathrm{Tg}$ C, respectively, in 2013) are calculated by the MEGAN-MOHYCAN model (Müller et al., 2008; Guenther et al., 2012; Bauwens et al., 2018) and are available online (http://emissions.aeronomie.be, last access: 3 June 2019). Biogenic emissions of acetaldehyde and ethanol (amounting to 92 and $88 \mathrm{Tg}(\mathrm{C}) \mathrm{yr}^{-1}$ globally) are parameterized as in Millet et al. (2010). The methanol biogenic emissions are provided by an inverse modelling study constrained by spaceborne methanol abundances and are estimated at $37.5 \mathrm{Tg}(\mathrm{C}) \mathrm{yr}^{-1}$ (Stavrakou et al., 2011). Biogenic emissions of $\mathrm{C}_{2} \mathrm{H}_{4}$ (scaled to a global total of $4 \mathrm{Tg}(\mathrm{C}) \mathrm{yr}^{-1}$ ), $\mathrm{CH}_{2} \mathrm{O}\left(1.6 \mathrm{Tg}(\mathrm{C}) \mathrm{yr}^{-1}\right)$, and $\mathrm{CH}_{3} \mathrm{C}(\mathrm{O}) \mathrm{CH}_{3}\left(18 \mathrm{Tg}(\mathrm{C}) \mathrm{yr}^{-1}\right)$ are also provided by MEGAN (Guenther et al., 2012) (available on http://eccad.aeris-data.fr, last access: 3 June 2019).

The model also includes oceanic emissions of methanol (18.4 $\left.\mathrm{Tg}(\mathrm{C}) \mathrm{yr}^{-1}\right)$, acetone (39.3 $\left.\mathrm{Tg}(\mathrm{C}) \mathrm{yr}^{-1}\right)$, and acetaldehyde (30.4 $\mathrm{Tg}(\mathrm{C}) \mathrm{yr}^{-1}$ ) (Müller et al., 2018), similar to previous model estimations (Stavrakou et al., 2011; Fischer et al., 2012; Millet et al., 2010). Finally, oceanic emissions of alkyl nitrates are also included, based on comparisons with aircraft campaign measurements as originally proposed by Neu et al. (2008) but taking into account the updated alkylnitrate calibration of the campaign data (Simpson et al., 2011). The adopted rates over tropical oceans $\left(10^{\circ} \mathrm{S}-10^{\circ} \mathrm{N}\right)$ are $6 \times 10^{8}, 2.5 \times 10^{8}, 10^{8}$, and $10^{8}$ molec. $\mathrm{cm}^{-2} \mathrm{~s}^{-1}$ for $\mathrm{C}_{1}, \mathrm{C}_{2}$, $\mathrm{C}_{3}$, and $\mathrm{C}_{>3}$ alkyl nitrates, respectively, and $3 \times 10^{7}, 3 \times 10^{7}$, $1.5 \times 10^{7}$, and $10^{7}$ molec. $\mathrm{cm}^{-2} \mathrm{~s}^{-1}$ over the Southern Ocean $\left(>10^{\circ} \mathrm{S}\right)$; a uniform rate of $10^{7}$ molec. $\mathrm{cm}^{-2} \mathrm{~s}^{-1}$ is adopted elsewhere over ice-free oceans. The calculated global emissions are, respectively, $0.35,0.3,0.2$, and $0.25 \mathrm{Tg}(\mathrm{C})$ or 0.4 , $0.18,0.08$, and $0.07 \mathrm{Tg}(\mathrm{N})$ for $\mathrm{C}_{1}, \mathrm{C}_{2}, \mathrm{C}_{3}$ and higher alkylnitrates.

MAGRITTE is run for a period of 18 months starting on 1 July 2012 , both at the global scale $\left(2^{\circ} \times 2.5^{\circ}\right.$ resolution $)$ and regional scale for the US $\left(0.5^{\circ} \times 0.5^{\circ}, 10-54^{\circ} \mathrm{N}, 65-\right.$ $\left.130^{\circ} \mathrm{W}\right)$. Only the results for the year 2013 are discussed hereafter.

\subsection{Model general results}

Oxidation of isoprene by $\mathrm{OH}$ radicals is by far the largest sink of isoprene, representing $\sim 85 \%$ of the global sink according to the model calculations, in agreement with previous model studies (Paulot et al., 2012), whereas ozonolysis and the $\mathrm{NO}_{3}$ reaction contribute $\sim 9 \%$ and $5 \%$, respectively. The isomerization reactions control the fate of about one-fifth of the to- 
tal flux of hydroperoxy radicals formed from the reaction of isoprene with $\mathrm{OH}(16.5 \%$ and $3 \%$ for the 1,6 and $1,5 \mathrm{H}$ shifts, respectively). However, the contribution of $1,6 \mathrm{H}$-shift is much higher, by about an order of magnitude, for the peroxys resulting from $\mathrm{OH}$ addition to carbon $\mathrm{C}_{4}$ than for those resulting from addition at $\mathrm{C}_{1}$ (Peeters et al., 2014; Wennberg et al., 2018). Furthermore, this contribution is dependent on temperature and on the concentrations of $\mathrm{NO}$ and $\mathrm{HO}_{2}$ radicals, as illustrated in Fig. 6: of the order of $50 \%$ over remote forests such as Amazonia, it drops to $~ 35 \%$ over the southeastern US and below $20 \%$ over cooler, more $\mathrm{NO}_{x}$-polluted areas (for $\mathrm{C}_{4}$ addition).

The isomerization reactions of isoprene peroxys regenerate $\mathrm{HO}_{x}\left(\mathrm{HO}_{2}+\mathrm{OH}\right)$ radicals, in part directly (see Sect. 2.1.2) and in part from subsequent reactions of the isomerization products, HPALDs in particular. However, as discussed in Sect. 3.1, the revised isomerization product distribution of the MAGRITTE v1.1 mechanism, consistent with recent experimental findings (Berndt et al., 2019), lowers the regeneration of $\mathrm{OH}$ compared with distributions assuming a large yield of $\mathrm{OH}$ radicals and dihydroperoxycarbonyls (Peeters et al., 2014; Wennberg et al., 2018) assumed to release additional $\mathrm{HO}_{x}$ through fast photolysis. Furthermore, our recently proposed enol-forming pathway in the fast photolysis of several key hydroperoxycarbonyls (e.g. HPACET and HPAC) also decreases the recycling of $\mathrm{OH}$ compared with the previous assumption of $\mathrm{O}-\mathrm{OH}$ bond scission. The overall impact of isoprene peroxy radical isomerization reactions on boundary-layer averaged $\mathrm{OH}$ concentrations reaches up to about $40 \%$ over western Amazonia and 10\%-15\% over the southeastern US and Siberia in July (Fig. 7), whereas their impact on $\mathrm{HO}_{2}$ is comparatively lower, as it does not exceed $20 \%$ over Amazonia. The isomerization reactions also lead to reduced isoprene nitrate formation, by up to $\sim 40 \%$ over Amazonia, as the $\mathrm{RO}_{2}+\mathrm{NO}$ reactions compete with unimolecular reactions. The decreased $\mathrm{NO}_{x}$ loss through organic nitrate formation and partial removal implies longer $\mathrm{NO}_{x}$ effective lifetime and higher concentrations (by a few $\%$ over Amazonia), in spite of the higher $\mathrm{OH}$ levels and increased $\mathrm{NO}_{x}$ loss through $\mathrm{NO}_{2}+\mathrm{OH}$. These changes lead to slightly enhanced $\mathrm{O}_{3}$ concentrations over Amazonia (a few percent). The impact on $\mathrm{HCHO}$ concentrations and vertically integrated columns is very small, also of the order of a few percent at most.

The dry or wet deposition of organic (peroxy)nitrates and the irreversible sink of organic nitrates through hydrolysis or other processes on aerosols are significant net sinks of $\mathrm{NO}_{x}$ over vegetated areas (Browne et al., 2014; Romer et al., 2016; Fisher et al., 2016). As shown in Fig. 8, the combined deposition and aerosol sink of organic (peroxy)nitrates is found to be the dominant sink of $\mathrm{NO}_{x}$ over rainforests in South America and Africa, as well as over boreal forests in Siberia and Canada during the summer. This fraction even exceeds $70 \%$ over the most remote areas (e.g. western Amazonia), where high isoprene and low $\mathrm{NO}_{x}$ levels both contribute to low $\mathrm{OH}$ concentrations (of the order of $10^{6}$ molec. $\mathrm{cm}^{-3}$ during daytime in the boundary layer). These estimates should be considered with caution given the large uncertainties in the assumed aerosol uptake coefficient and poor understanding of aerosol chemical processes. Over the southeastern US $\left(29.5-40^{\circ} \mathrm{N}, 80-94.5^{\circ} \mathrm{W}\right)$ during August-September 2013, the MAGRITTE model calculations (regional version over the US, $0.5^{\circ}$ resolution) suggest that the $\mathrm{NO}_{x}$ sink through aerosol hydrolysis amounts to $14 \%$ of $\mathrm{NO}_{x}$ emissions in the region, whereas the deposition of organic nitrates and peroxynitrates account for additional $7 \%$ and $5 \%$ of $\mathrm{NO}_{x}$ emissions. The estimated total net loss of $\mathrm{NO}_{x}$ through $\mathrm{RONO}_{2}$ formation amounts, therefore, to $21 \%$ of $\mathrm{NO}_{x}$ emissions, is in good agreement with previous calculations using the GEOS-Chem model (Fisher et al., 2016) (also 21\%). This agreement might be partly fortuitous, given the important differences between the two studies regarding the nitrate yield in the ISOPO2 + NO reactions (9\% in Fisher et al., 2016 and $13 \%$ in our study) and regarding the treatment of $\mathrm{RONO}_{2}$ aerosol sink: a unique uptake coefficient $(0.005)$ was used by Fisher et al. (2016) for all isoprene nitrates except nitroxyacetone and ethanal nitrate, whereas only tertiary nitrates are assumed to undergo aerosol hydrolysis in our study (with $\gamma=0.03$ ). Non-tertiary nitrates might partition to the aerosol phase and possibly undergo processes preventing their eventual release to the gas-phase, in which case the overall $\mathrm{NO}_{x}$ sink calculated here is underestimated.

Although SOA is not a focus of this study, SOA formation processes are included in the model. The largest source of SOA is the uptake of IEPOX, with a global flux (49 Tg or $25 \mathrm{Tg} \mathrm{C} \mathrm{yr}^{-1}$ ) of magnitude similar to previous model estimates of the order of $40 \mathrm{Tg} \mathrm{yr}^{-1}$ (Lin et al., 2012; Stadtler et al., 2018). These estimates are very uncertain, since the reactive uptake parameterization used in models ignores the complexity of SOA formation, which involves the partitioning of semi-volatile compounds and chemical transformations in the gaseous and particulate phases (D'Ambro et al., 2019). Glyoxal is another well-identified source of SOA, amounting to $10 \mathrm{Tg} \mathrm{yr}^{-1}$ globally $\left(4.3 \mathrm{Tg} \mathrm{Cyr}^{-1}\right)$, also well in the range of previous estimations $\left(6-14 \mathrm{Tg} \mathrm{yr}^{-1}\right.$ ) (Fu et al., 2008; Stavrakou et al., 2009b; Lin et al., 2012). The dihydroxy dihydroperoxides (ISOP $(\mathrm{OOH})_{2}$ ) formed from the oxidation of ISOPOOH by $\mathrm{OH}$ were recently estimated to be a dominant source of SOA (Stadtler et al., 2018); in our mechanism, these compounds are ignored since their yields are believed to be negligible in atmospheric conditions (D'Ambro et al., 2017). The major non-IEPOX products of $\mathrm{OH}$ addition to ISOPOOH are dihydroxy hydroperoxy epoxides (DHHEPOX), also believed to form SOA as discussed above (Note N6). Their global production in the model amounts to $30 \mathrm{Tg} \mathrm{yr}^{-1}\left(12 \mathrm{TgC} \mathrm{yr}^{-1}\right)$. Assuming that their reactive uptake is as effective as for IEPOX and neglecting gas-phase oxidation by $\mathrm{OH}$ (which generates other low-volatility compounds also expected to form SOA), we estimate with the model that SOA formation accounts for two-thirds of the 
(a) 1,6 isom. fraction, C1-addition

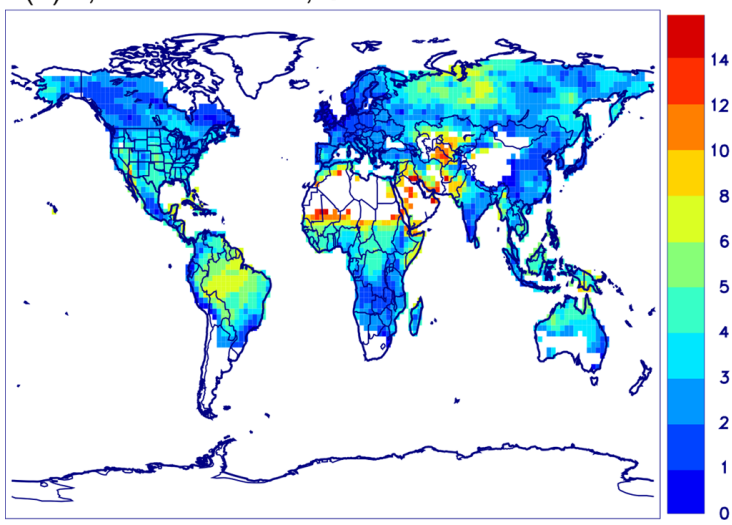

(b) 1,6 isom. fraction, C4-addition

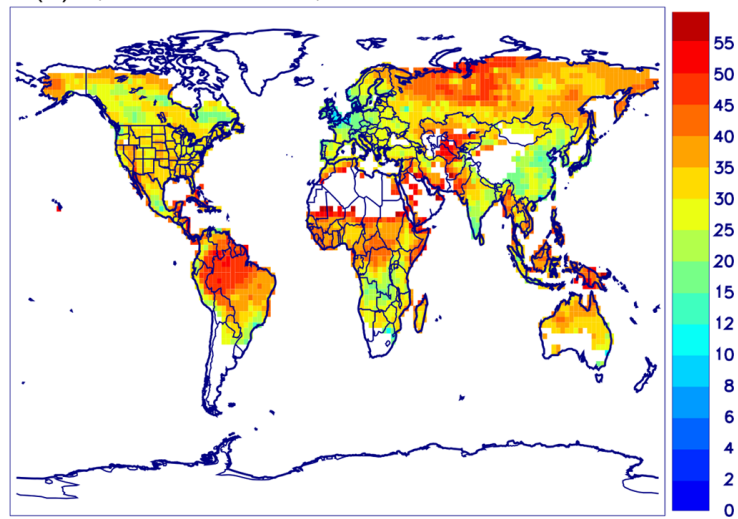

Figure 6. Calculated percentage contribution of $Z$ - $\delta$-hydroxyperoxy 1,6 H-shift to the overall sink of the pool of peroxys resulting from addition of $\mathrm{OH}$ (a) to $\mathrm{C}_{1}$, and (b) to $\mathrm{C}_{4}$ of isoprene (column average, July 2013). Note the different colour scales in panels (a) and (b).

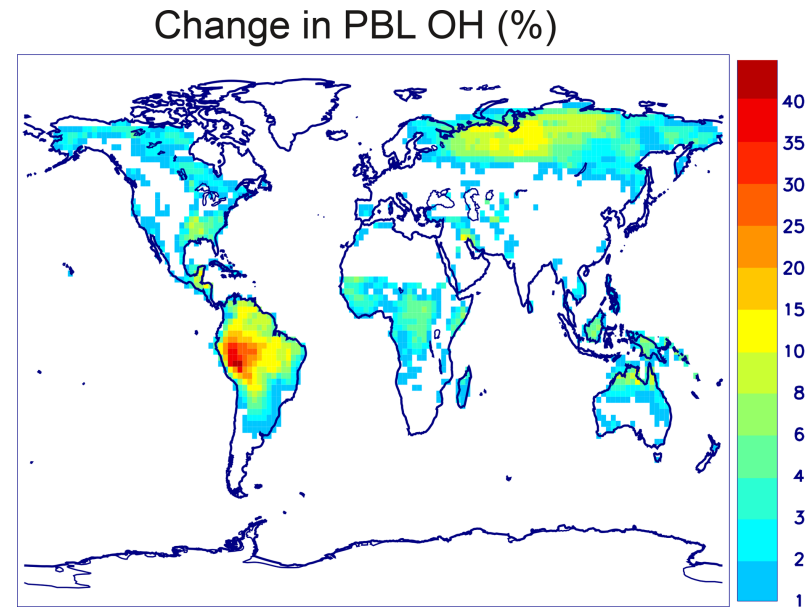

Figure 7. Calculated change (in \%) in boundary layer $\mathrm{OH}$ concentration upon inclusion of isomerization reactions of isoprene peroxy radicals (column average, July 2013).

sink of DHHEPOX (i.e. $20 \mathrm{Tg} \mathrm{yr}^{-1}$ ), whereas dry and wet deposition makes up the rest. If confirmed, this would make DHHEPOX the second-largest contributor to isoprene SOA.

Other SOA formation pathways are implied but not explicitly represented by the MAGRITTE mechanism, such as the hydrolysis of dihydroxy dinitrates (Note N12) and dihydroxy hydroperoxy nitrates (Note N13). The hydrolysis products, nitroxy- and hydroperoxy triols are expected to be of very low volatility and remain mostly in the aerosol phase, as their vapour pressures (Compernolle et al., 2011) are estimated to be very low. Those triols represent only a minor contribution to the global SOA budget, however, as their estimated global

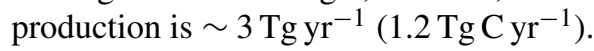

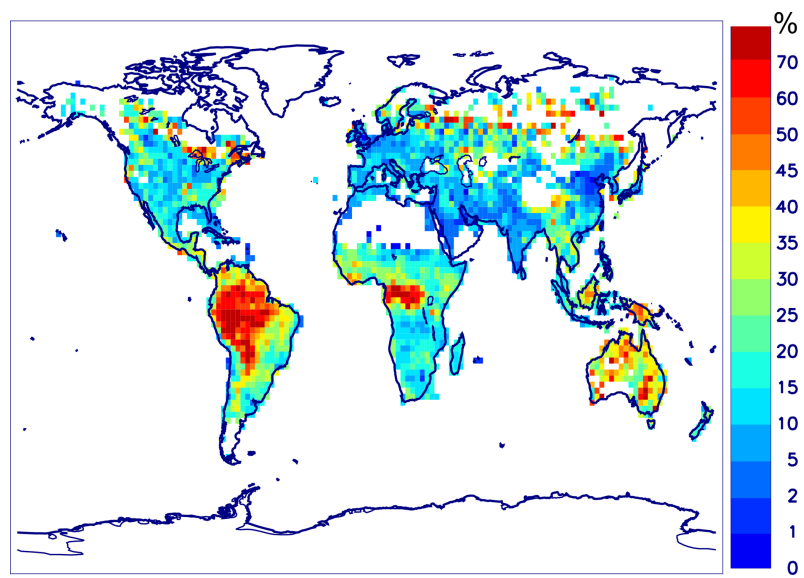

Figure 8. Percentage ratio of annual $\mathrm{NO}_{x}$ net loss due to organic nitrate formation (i.e. their combined aerosol sink and deposition sink) to the total annual $\mathrm{NO}_{x}$ emission. Blank areas are those with annually averaged $\mathrm{NO}_{x}$ emissions lower than $5 \times$ $10^{9}$ molec. $\mathrm{cm}^{-2} \mathrm{~s}^{-1}$.

\subsection{Model evaluation against $\mathrm{SEAC}^{4} \mathrm{RS}$ campaign measurements}

The regional model simulation over the US is evaluated against aircraft measurements of the NASA SEAC ${ }^{4} \mathrm{RS}$ (Studies of Emissions and Atmospheric Composition, Clouds and Climate Coupling by Regional Surveys) campaign in August-September 2013 (Toon et al., 2016). For the most part, the SEAC ${ }^{4} \mathrm{RS}$ took place over the southeastern US in areas characterized by high emissions of isoprene and other BVOCs. The observations discussed below are those obtained on the NASA DC-8 (http://www-air. larc.nasa.gov/missions/merges/, last access: 3 June 2019) between 09:00 and 17:00 LT. Biomass burning plumes, urban plumes, and stratospheric air are excluded from the analysis 
(diagnosed with $\left[\mathrm{CH}_{3} \mathrm{CN}\right]>225 \mathrm{ppt},\left[\mathrm{NO}_{2}\right]>4 \mathrm{ppbv}$, and $\left[\mathrm{O}_{3}\right] /[\mathrm{CO}]>1.25$, respectively) (Travis et al., 2016).

Figure 9 presents the observed and calculated average profiles of ozone, $\mathrm{NO}_{x}$, and VOC oxidation products. The model profiles are averages based on values interpolated at each measurement location and time. As noted above, the $\mathrm{NO}_{x}$ anthropogenic emissions used in the model were strongly reduced, relative to NEI official estimations, in order to match the SEAC ${ }^{4} \mathrm{RS}$ observations for $\mathrm{NO}_{2}$ (also $\mathrm{NO}$ ) and improve the agreement for ozone, consistent with the results of Travis et al. (2016). The model is in excellent agreement with the $\mathrm{HCHO}$ profile measured by the Compact Atmospheric Multispecies Spectrometer (CAMS) (Richter et al., 2015), with only about $3 \%$ average overestimation below $4 \mathrm{~km}$ altitude, whereas a model underestimation of $8 \%$ is found relative to $\mathrm{HCHO}$ measurements by laser-induced fluorescence (NASA GSFC ISAF instrument, Cazorla et al., 2015, not shown in Fig. 9). The model performance is also fairly good for the major products of isoprene $+\mathrm{OH}$, with moderate overestimations of $14 \%, 1 \%$, and $24 \%$ for MVK + MACR, ISOPN (the family of primary hydroxynitrates from isoprene), and ISOPOOH, respectively. Even for ISOPOOH, the model falls well within the measurement uncertainty range (40\%) (Nguyen et al., 2015b). Note that the modelled MVKMAC accounts for the presumed interference of ISOPOOH in the measurement, as described in Müller et al. (2018). This correction increases MVKMAC by $\sim 10 \%$ on average for this campaign.

The model-calculated HPALD concentrations (dotted line on the $\mathrm{C}_{5} \mathrm{H}_{8} \mathrm{O}_{3}$ panel of Fig. 9) are on average about a factor of 2 lower than the observed Caltech CIMS (Chemical Ionization Mass Spectrometry) signal at the corresponding mass; when adding the contribution of the carbonyl hydroxyepoxides (ICHE), which have the same formula $\left(\mathrm{C}_{5} \mathrm{H}_{8} \mathrm{O}_{3}\right)$ as HPALD and can be expected to interfere with HPALD measurements, the model falls within the measurement uncertainty range $(50 \%)$ with an underestimation decreased to $-34 \%$ (solid line in Fig. 9). The ICHE compounds are formed from the oxidation of IEPOX (as well as HPALDs) by $\mathrm{OH}$. It is likely that other, unknown compounds contribute to the CIMS signal at the same mass, as also observed in the PROPHET campaign in Michigan, where the HPALD contribution to the CIMS measurement at the given mass was estimated at $38 \%$ based on the relative contribution of the HPALD peaks to the total GC area (Vasquez et al., 2018). This is consistent with our modelled HPALD accounting for $50 \%$ of the CIMS measurement, when also considering that all isoprene oxidation products appear slightly overestimated by the model, as suggested by the $\sim 20 \%$ overprediction of modelled ISOPOOH and MVK+MACR relative to the measurements. In spite of the important uncertainties and remaining unknowns (e.g. the identity of additional compounds contributing to the CIMS signal), this good consistency provides strong support to the high HPALD yield $(75 \%)$ adopted in this work in the isomerization of $Z-\delta$-OH-peroxys from iso- prene (Sect. 2.1.2). Lower yield values, as proposed in recent previous work, i.e. 50\% (Peeters et al., 2014; Jenkin et al., 2015) or $25 \%$ (Teng et al., 2017; Wennberg et al., 2018) would lead to much stronger HPALD underestimations against $\mathrm{SEAC}^{4} \mathrm{RS}$ data.

The good consistency between the model results for the major high- $\mathrm{NO}_{x}$ and low- $\mathrm{NO}_{x}$ isoprene oxidation products lends confidence in the major steps of the mechanism. The excellent agreement for IEPOX $(+2 \%$ bias below $4 \mathrm{~km}$ ) might be partly fortuitous given the highly uncertain aerosol sink $(\sim 35 \%$ of the total IEPOX sink in the model simulation), without which the model would largely overestimate IEPOX observations. The slightly too low ISOPN/MVKMAC ratio in the model (0.036 vs. 0.041 ) could indicate an overestimation of ISOPN aerosol sink, although the measurement uncertainties $(\sim 30 \%$ for ISOPN, Fisher et al., 2016) preclude a firm assessment. Aerosol hydrolysis represents $\sim 50 \%$ of the total sink of the tertiary hydroxynitrate ISOPBNO3 in the model (average over the model domain) or about $31 \%$ of the total ISOPN sink. The model overestimation of the secondary isoprene nitrates (MVKNO3+MACRNO3) (Fig. 9) is small (14\%) and suggests an essentially correct representation of their sources and sinks, although error compensations remain a possibility. The model overestimates nitroxyacetone (NOA) by $\sim 170 \%$, in contrast with the GEOS-Chem underestimation found by Fisher et al. (2016). This compound is mainly produced from multiple reaction sequences in the $\mathrm{NO}_{3}$-initiated oxidation mechanism of isoprene and in the $\mathrm{OH}$-oxidation mechanism of the $\delta$-hydroxynitrate $\mathrm{HOCH}_{2} \mathrm{CH}=\mathrm{C}\left(\mathrm{CH}_{3}\right) \mathrm{CH}_{2} \mathrm{ONO}_{2}$ (ISOPCNO3). Although isoprene oxidation by $\mathrm{NO}_{3}$ is primarily a night-time process, NOA is formed after several oxidation steps favoured by daylight. Our mechanism is more detailed and in line with the recent mechanistic conclusions from laboratory studies, but it still bears large uncertainties due to the high complexity of the mechanism. For example, the $\mathrm{H}$-shift in the nitroxyperoxy radical INCO2 $\left(\mathrm{HOCH}_{2} \mathrm{CH}(\mathrm{OH}) \mathrm{C}\left(\mathrm{O}_{2}\right)\left(\mathrm{CH}_{3}\right) \mathrm{CH}_{2} \mathrm{ONO}_{2}\right.$ and isomer) leads to NOA formation according to our mechanism. Although this process is written as one reaction in the mechanism, it actually involves several steps, each of which is uncertain. The model might also overestimate nitrate radical concentrations and, therefore, also the importance of $\mathrm{NO}_{3}$ as oxidant of isoprene. Although the reactions of $\mathrm{NO}_{3}$ with major peroxy radicals and carbonyls are taken into account in the model, many reactions with unsaturated oxidation products (e.g. ISOPOOH) are neglected in current mechanisms. A careful assessment of the role of these reactions might be in order.

Despite the model overestimation for NOA, the model underestimates the SEAC ${ }^{4} \mathrm{RS}$ measurement for $\mathrm{RONO}_{2}$ (the sum of all organic nitrates) by $\sim 40 \%$. A slightly larger model underestimation (factor of 2) was found by Fisher et al. (2016), in line with their lower $\mathrm{RONO}_{2}$ yield in the ISOPO2 + NO reactions (see above). There are several pos- 

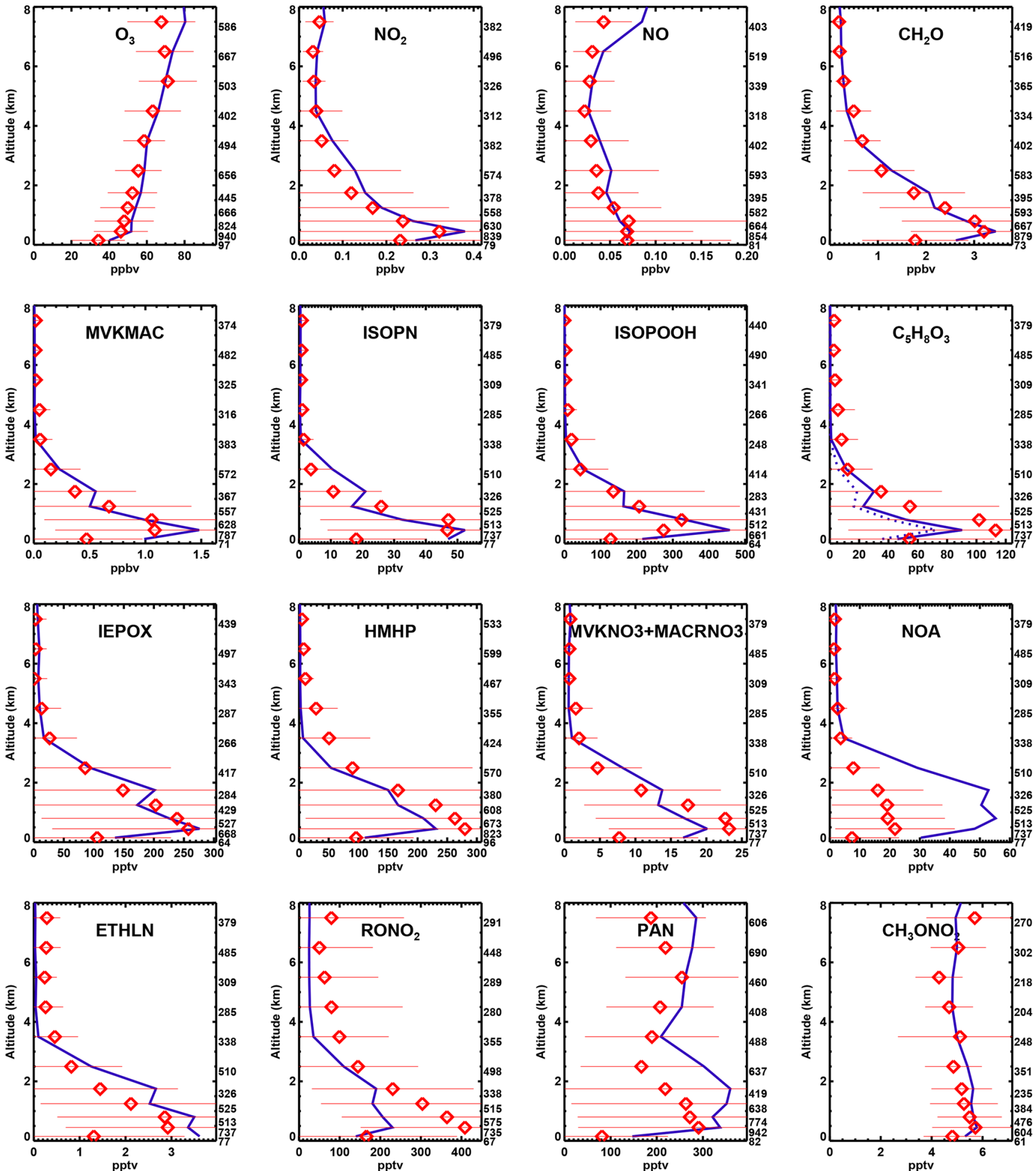

Figure 9. Observed (red symbols) and modelled (black lines) mean profiles of ozone, $\mathrm{NO}_{2}$, $\mathrm{NO}$, and major VOC oxidation products over North America during the SEAC ${ }^{4} \mathrm{RS}$ campaign. The number of measurements per altitude bin is indicated on the right for each plot. The vertical bin interfaces are $0,0.3,0.6,1,1.5 \mathrm{~km}$, and from 2 to $8 \mathrm{~km}$ by $1 \mathrm{~km}$. The horizontal lines indicate the standard deviation of the measurements within each vertical bin. MVKMAC stands for the sum MVK+ MACR + 0.44ISOPOOH. Both the modelled HPALD (dotted line) and HPALD + ICHE (solid line) are shown in the $\mathrm{C}_{5} \mathrm{H}_{8} \mathrm{O}_{3}$ panel. 
sible explanations for the discrepancy, including the neglected reactions of $\mathrm{NO}_{3}$ with unsaturated oxidation products from isoprene and other BVOCs, the neglected formation of unsaturated dinitrates from the reaction of dinitroxyperoxy radicals (NISOPO2) with NO (Li et al., 2018), a possible overestimate of the tertiary nitrate hydrolysis sink (for dinitrates in particular) and a misrepresentation of alkyl and hydroxyalkyl nitrates from precursors other than isoprene. The monoterpene nitrates are very crudely represented in the model. In particular, the assumption of $100 \% \mathrm{NO}_{x}$ recycling in their reaction with $\mathrm{OH}$ could lead to a significant overestimation of $\mathrm{RONO}_{2}$ loss. Nitrates from ethane, propane, ethene, and propene oxidation are included in MAGRITTE, but their concentrations are largely underestimated with respect to SEAC ${ }^{4}$ RS observations (not shown in Fig. 9), in part due to underestimations of precursors emissions, for ethane, propane, and propene in particular. However, these nitrates account for only a small part of the $\mathrm{RONO}_{2}$ bias $(\sim 16 \mathrm{pptv}$ altogether out of 120 pptv below $4 \mathrm{~km}$ ) based on SEAC ${ }^{4} \mathrm{RS}$ observations and model results. Nitrates from higher alkanes are crudely included in the model, and their contribution could be underestimated. Methylnitrate $\left(\mathrm{CH}_{3} \mathrm{ONO}_{2}\right)$ is well reproduced by the model (Fig. 9), but it makes only a very small contribution $(\sim 5 \mathrm{ppt})$. The good agreement validates the low nitrate yield used in the mechanism $\left(2 \times 10^{-4}\right.$ at room conditions; see Note N71) for the $\mathrm{CH}_{3} \mathrm{O}_{2}+\mathrm{NO}$ reaction, well below the experimental determination $(1 \% \pm 0.7 \%$ in tropospheric conditions) of Butkovskaya et al. (2012). Although a higher yield $\left(\sim 3 \times 10^{-4}\right)$ would still remain compatible with the SEAC ${ }^{4} \mathrm{RS}$ measurement (by assuming lower oceanic emissions), much higher values as reported by Butkovskaya et al. (2012) would lead to huge overestimations of $\mathrm{CH}_{3} \mathrm{ONO}_{2}$ mixing ratios in the troposphere.

\subsection{Global budget of formic and acetic acid}

The calculated global photochemical source of formic acid amounts to $5.6 \mathrm{Tg} \mathrm{C}$ or $21 \mathrm{Tg}(\mathrm{HC}(\mathrm{O}) \mathrm{OH})$ per year (Table 5). Although the model simulation incorporates newly proposed formation mechanisms, as detailed below, this total is lower than several previous model estimations (Paulot et al., 2011; Stavrakou et al., 2012; Millet et al., 2015), for several reasons. Firstly, the global isoprene source in our simulation $\left(366 \mathrm{Tg} \mathrm{Cyr}^{-1}\right)$ is near the low end of the range of previous estimates (Arneth et al., 2011; Sindelarova et al., 2014). Furthermore, the formation of $\mathrm{HC}(\mathrm{O}) \mathrm{OH}$ in the oxidation of glycolaldehyde and hydroxyacetone implemented in several studies is omitted here, since the original experimental findings by Butkovskaya et al. (2006a, b) could not be confirmed (Orlando et al., 2012) and might not be effective in atmospheric conditions. $\mathrm{HC}(\mathrm{O}) \mathrm{OH}$ production from isoprene ozonolysis $\left(1 \mathrm{Tg} \mathrm{Cyr}^{-1}\right)$ is lower than previous estimates (e.g. 1.8 and $2.3 \mathrm{Tg} \mathrm{C} \mathrm{yr}^{-1}$ in Paulot et al., 2011 and Stavrakou et al., 2012, respectively) despite our high assumed yield (0.58) of stabilized Criegee $\left(\mathrm{CH}_{2} \mathrm{OO}\right)$. This is
Table 5. Global sources of $\mathrm{HC}(\mathrm{O}) \mathrm{OH}$ in the model simulation. Roman font is used for the main sources, whereas subtotals are shown in italic font.

\begin{tabular}{|c|c|c|}
\hline & $\operatorname{Tg}(\mathrm{C}) \mathrm{yr}^{-1}$ & $\mathrm{Tg}(\mathrm{HC}(\mathrm{O}) \mathrm{OH}) \mathrm{yr}^{-1}$ \\
\hline \multicolumn{3}{|l|}{ Direct emissions } \\
\hline Biomass burning & 0.78 & 3.0 \\
\hline Biogenic & 1.46 & 5.6 \\
\hline Anthropogenic & 0.58 & 2.2 \\
\hline \multicolumn{3}{|l|}{ Photochemical production } \\
\hline $\mathrm{ISOP}+\mathrm{O}_{3}$ & 0.95 & 3.6 \\
\hline Other alkenes ozonolysis & 0.52 & 2.0 \\
\hline $\mathrm{C}_{2} \mathrm{H}_{2}+\mathrm{OH}$ & 0.69 & 2.6 \\
\hline $\mathrm{APIN}+\mathrm{OH}$ & 0.41 & 1.6 \\
\hline $\mathrm{VA}+\mathrm{OH}$ & 1.66 & 6.4 \\
\hline from $\mathrm{CH}_{3} \mathrm{CHO}+\mathrm{h} v$ & 0.76 & 2.9 \\
\hline from $\mathrm{OCHCH}_{2} \mathrm{OOH}+\mathrm{h} v$ & 0.90 & 3.4 \\
\hline $\mathrm{ISOP}+\mathrm{OH}$ (various pathways) & 1.36 & 5.2 \\
\hline $\mathrm{HMAC} / \mathrm{HMVK}+\mathrm{OH}$ & 0.91 & 3.5 \\
\hline $\mathrm{ISOPOOH}+\mathrm{OH}$ & 0.44 & 1.7 \\
\hline
\end{tabular}

Total source

Global 8.4 32

due to the combination of (1) low direct formation yield of $\mathrm{HC}(\mathrm{O}) \mathrm{OH}$ in the $\mathrm{CH}_{2} \mathrm{OO}$ reaction with the water dimer (Sheps et al., 2017), (2) high deposition sink of HMHP (over $\sim 50 \%$ of its global production) resulting from its high solubility and high deposition velocities over forests (Nguyen et al., 2015b; Müller et al., 2018), and (3) the $\mathrm{HC}(\mathrm{O}) \mathrm{OH}$ yield of only 0.45 in the reaction of HMHP with $\mathrm{OH}$ recently estimated from experiment (Allen et al., 2018). The very good model agreement against the SEAC ${ }^{4} \mathrm{RS}$ measurements of HMHP over the southeastern US suggests an essentially correct model representation of its production and sink rate, and, therefore, of the contribution of alkene ozonolysis to the budget of formic acid.

Vinyl alcohol (VA), originally proposed as possible source of formic acid by Archibald et al. (2007), received full attention when acetaldehyde phototautomerization to VA was shown in the laboratory to be efficient (Andrews et al., 2012) and to represent a sizable source of formic acid of the order of $3 \mathrm{Tg} \mathrm{C} \mathrm{yr}^{-1}$ (Cady-Perreira et al., 2014; Millet et al., 2015). However, a recent, more detailed experimental evaluation of the phototautomerization yield led to a downward revision of the global source to about $0.8 \mathrm{Tg} \mathrm{C} \mathrm{yr}^{-1}$ (Shaw et al., 2018), in good agreement with our model calculations (Table 5). This source could be even lower if VA tautomerizes back to acetaldehyde (da Silva et al., 2010), but acid-catalysed VA tautomerization was shown to be negligible, and aerosolmediated tautomerization remains speculative (Peeters et al., 2015).

Another source of VA and of other enols has been identified: the photolysis of hydroperoxycarbonyls (Liu et al., 2018). Our results (Table 5) indicate that the photolysis of 
hydroperoxyacetaldehyde (HPAC) is a larger source of VA (and, therefore, of $\mathrm{HC}(\mathrm{O}) \mathrm{OH}$ ) than $\mathrm{CH}_{3} \mathrm{CHO}$ tautomerization. The sources of HPAC (4.7 $\mathrm{Tg} \mathrm{yr}^{-1}$ globally) include the oxidation of acetaldehyde by $\mathrm{OH}$ ( $35 \%$ of total), the photolysis of MVKOOH (35\%) and several other pathways in isoprene oxidation, in particular through the isoprene hydroxyperoxy radical 1,6 $\mathrm{H}$-shift pathway. In addition, the photolysis of the HPALDs, of $\mathrm{C}_{4}$ hydroperoxy dicarbonyls (HPDIAL and HPKETAL) also generated from the isomerization pathway, and of nitroxyenals (NC4CHO) formed from isoprene $+\mathrm{NO}_{3}$, all lead partly to keto-enols (HMAC and $\mathrm{HMVK})$, which are oxidized in large part into $\mathrm{HC}(\mathrm{O}) \mathrm{OH}$ following their reaction with $\mathrm{OH}$, adopting a similar mechanism as for VA (So et al., 2014). The photolysis and deposition of HMVK and HMAC are found to be minor sinks ( 5\% and $10 \%$ of their global sink, respectively). Finally, hydroperoxycarbonyls formed from minor pathways in the ISOPOOH degradation mechanism are photolysed in part into other enol compounds, which are partly oxidized to $\mathrm{HC}(\mathrm{O}) \mathrm{OH}$ (along with MVK or MACR). The estimated combined $\mathrm{HC}(\mathrm{O}) \mathrm{OH}$ source due to hydroperoxycarbonyl photolysis amounts to $2.25 \mathrm{TgC}^{-1}$, exceeding in magnitude the source due to alkene ozonolysis $\left(1.5 \mathrm{Tg} \mathrm{C} \mathrm{yr}^{-1}\right)$. As seen in Fig. 10a, the contribution of this source to near-surface $\mathrm{HC}(\mathrm{O}) \mathrm{OH}$ concentrations is highest over remote oceanic areas (up to $50 \%$ ) and is comparatively much lower over biomass burning and biogenic emission areas. This is partly due to HPAC formation due to oceanic acetaldehyde emissions, and partly due to the significant share of direct biogenic and pyrogenic emissions to the global $\mathrm{HC}(\mathrm{O}) \mathrm{OH}$ budget (Table 5). Nevertheless, hydroperoxycarbonyl photolysis enhances $\mathrm{HC}(\mathrm{O}) \mathrm{OH}$ levels by $\sim 20 \%$ (up to 150 pptv) near the surface over vegetated areas such as Amazonia (Fig. 10a), and by $>30 \%$ at higher tropospheric levels (not shown).

The largest known photochemical source of $\mathrm{CH}_{3} \mathrm{C}(\mathrm{O}) \mathrm{OH}$ is the reaction of acetylperoxy radical $\mathrm{CH}_{3} \mathrm{C}(\mathrm{O}) \mathrm{O}_{2}$ with peroxy radicals $\left(\mathrm{HO}_{2}\right.$ and $\left.\mathrm{RO}_{2}\right)$, amounting to $\sim 16 \mathrm{Tg} \mathrm{C} \mathrm{yr}^{-1}$ globally (Table 6). This is very consistent with a previous model estimate (18 $\mathrm{Tg} \mathrm{Cyr}^{-1}$ ) by Paulot et al. (2011) but significantly lower than the estimate of Khan et al. (2018) (close to $30 \mathrm{Tg} \mathrm{C} \mathrm{yr}^{-1}$ ). Our calculated contribution of $\mathrm{CH}_{3} \mathrm{C}(\mathrm{O}) \mathrm{O}_{2}+\mathrm{RO}_{2}$ reactions $\left(\sim 2.3 \mathrm{Tg} \mathrm{C} \mathrm{yr}^{-1}\right)$ is smaller than in Paulot et al. (2011) $\left(\sim 5.6 \mathrm{Tg} \mathrm{C} \mathrm{yr}^{-1}\right)$. It could be underestimated if the $\mathrm{CH}_{3} \mathrm{C}(\mathrm{O}) \mathrm{OH}$-forming channel ratio for the reactions of $\mathrm{CH}_{3} \mathrm{C}(\mathrm{O}) \mathrm{O}_{2}$ with major non-tertiary peroxy radicals would be significantly higher than the value assumed here for most reactions $(0.1)$, which is based on the case of $\mathrm{CH}_{3} \mathrm{C}(\mathrm{O}) \mathrm{O}_{2}+\mathrm{CH}_{3} \mathrm{O}_{2}$ (Atkinson et al., 2006). The high reported $\mathrm{CH}_{3} \mathrm{C}(\mathrm{O}) \mathrm{OH}$ yield (0.5) (Atkinson et al., 2006) in the case of $\mathrm{CH}_{3} \mathrm{C}(\mathrm{O}) \mathrm{O}_{2}+\mathrm{CH}_{3} \mathrm{C}(\mathrm{O}) \mathrm{CH}_{2} \mathrm{O}_{2}$ is implemented in our mechanism but assumed to be atypical.

The additional source of acetic acid due to the photolysis of hydroperoxyacetone (HPACET) and involving the oxidation of methyl vinyl alcohol (MVA) by $\mathrm{OH}$ enhances the estimated global photochemical production of $\mathrm{CH}_{3} \mathrm{C}(\mathrm{O}) \mathrm{OH}$
Table 6. Global sources of $\mathrm{CH}_{3} \mathrm{C}(\mathrm{O}) \mathrm{OH}$ in the model simulation. Roman font is used for the main sources, whereas subtotals are shown in italic font.

\begin{tabular}{lrr}
\hline & $\mathrm{Tg}(\mathrm{C}) \mathrm{yr}^{-1}$ & $\mathrm{Tg}\left(\mathrm{CH}_{3} \mathrm{C}(\mathrm{O}) \mathrm{OH}\right) \mathrm{yr}^{-1}$ \\
\hline Direct emissions & & \\
\hline Biomass burning & 5.7 & 14.3 \\
Anthropogenic & 2.6 & 6.6 \\
\hline Photochemical production & & \\
\hline $\mathrm{CH}_{3} \mathrm{C}(\mathrm{O}) \mathrm{O}_{2}+\mathrm{HO}_{2}$ & 14.0 & 35.0 \\
$\mathrm{CH}_{3} \mathrm{C}(\mathrm{O}) \mathrm{O}_{2}+\mathrm{RO}_{2}$ & 2.3 & 5.7 \\
$\mathrm{HPACET}+\mathrm{h} v(+\mathrm{OH})$ & 4.3 & 10.9 \\
$\quad$ from isoprene oxidation & 2.1 & 5.2 \\
$\quad$ from acetone oxidation & 1.5 & 3.8 \\
$\quad$ other & 0.7 & 1.8 \\
Other & 0.2 & 0.5 \\
\hline Total source & & 73 \\
\hline Global & 29.1 & \\
\hline
\end{tabular}

by $4.3 \mathrm{TgC}_{\mathrm{yr}}{ }^{-1}$ or $26 \%$ (Table 6 ). The global source of HPACET ( $23 \mathrm{Tg} \mathrm{C} \mathrm{yr}^{-1}$ ) is dominated by the acetonylperoxy radical reaction with $\mathrm{HO}_{2}\left(15 \mathrm{Tg} \mathrm{C} \mathrm{yr}^{-1}\right)$ and by the isoprene peroxy isomerization pathway $\left(2.4 \mathrm{Tg} \mathrm{C} \mathrm{yr}^{-1}\right.$ through the 1,4 $\mathrm{H}$-shift of DIHPCARP2 and 2.7 $\mathrm{Tg} \mathrm{C} \mathrm{yr}^{-1}$ from the photooxidation of carbonyl hydroperoxyepoxides ICPE). The precise mechanisms for the formation of HPACET (also HPAC) in the isomerization pathway remain uncertain. Photolysis accounts for $69 \%$ of the global HPACET sink, whereas reaction with $\mathrm{OH}$ and deposition account for $26 \%$ and $5 \%$, respectively. The only significant sink of MVA, the main product of HPACET photolysis, is reaction with $\mathrm{OH}$, assumed to form $\mathrm{CH}_{3} \mathrm{C}(\mathrm{O}) \mathrm{OH}$ (along with $\mathrm{OH}$ and $\mathrm{HCHO}$ ) with a $50 \%$ yield, following a mechanism similar to that for $\mathrm{VA}+\mathrm{OH}$ (So et al., 2014). The calculated contribution of HPACET photolysis to the $\mathrm{CH}_{3} \mathrm{C}(\mathrm{O}) \mathrm{OH}$ concentration (Fig. 10b) is highest over forests (except in areas impacted by biomass burning), up to $23 \%$ (120 pptv) over the southeastern US, and $30 \%$ (120 pptv) over Amazonia.

Despite the newly proposed large production of formic and acetic through hydroperoxycarbonyl photolysis, our derived total sources of those acids remains similar to (or even lower than) in previous modelling studies (Paulot et al., 2011; Stavrakou et al., 2012; Millet et al., 2015; Khan et al., 2018), and is, therefore, insufficient to explain their high observed concentrations. Additional sources are likely at play, such as enol formation through other pathways than those considered here, such as in monoterpene and anthropogenic VOC oxidation, e.g. through the photolysis of aldehydes (Tadic et al., 2001a, b) and the photodegradation of organic aerosols (Paulot et al., 2011; Malecha and Nizkodorov, 2016). 
(a) Contribution to near-surface $\mathrm{HCOOH}(\%)$

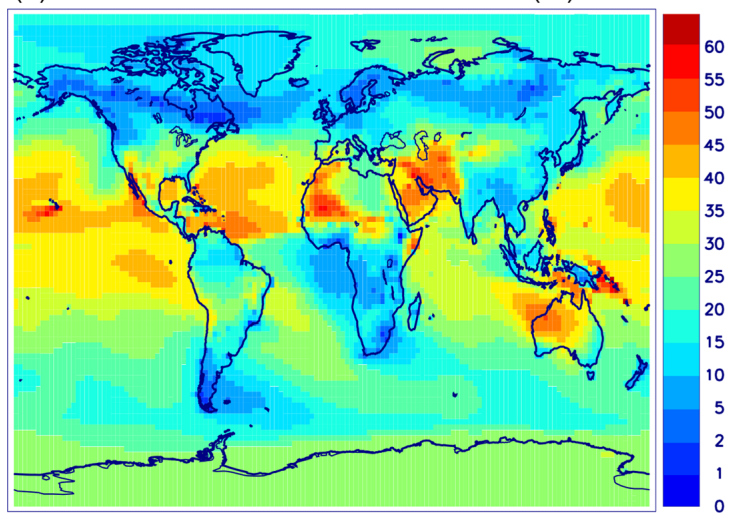

(b) Contribution to near-surface $\mathrm{CH} 3 \mathrm{COOH}(\%)$

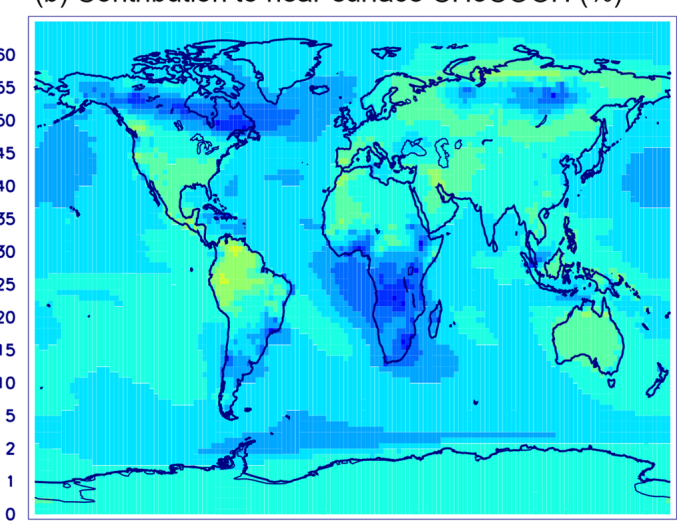

Figure 10. Calculated percentage contribution of hydroperoxycarbonyl photolysis to near-surface concentrations of (a) formic and (b) acetic acid for the month of July.

\subsection{Global budget of glyoxal}

The global sources of glyoxal as calculated by the model are summarized in Table 7. The model includes an important contribution from (mostly anthropogenic) acetylene and aromatic compounds to the glyoxal budget. The glyoxal yields in their reactions with $\mathrm{OH}(0.74,0.7,0.36$, and 0.636 for benzene, toluene, xylenes, and acetylene, respectively) are obtained from the MCM (Saunders et al., 2003; Bloss et al., 2005). Regarding aromatics, this yield includes not only primary formation but also later-generation production (Chan Miller et al., 2016). Contrary to previous model evaluations (Fu et al., 2008; Stavrakou et al., 2009b; Li et al., 2016; Chan Miller et al., 2017; Silva et al., 2018), isoprene oxidation is not found to be a very large source of glyoxal, except for the significant contribution of glycolaldehyde oxidation by $\mathrm{OH}$, which amounts to $\sim 4.7 \mathrm{Tg} \mathrm{Cyr}^{-1}$ of glyoxal. This has several causes. The oxidation of isoprene by $\mathrm{NO}_{3}$ is now an almost negligible glyoxal source in our mechanism (as in the Caltech mechanism), whereas an overall yield of $35 \%$ glyoxal was inferred from the MCMv3.2 mechanism (Stavrakou et al., 2009b). First-generation glyoxal formation from ISOP $+\mathrm{OH}$ with a yield of $\sim 2 \%$ at high $\mathrm{NO}_{x}$ through the $\delta-\mathrm{ISOPO} 2+\mathrm{NO} \rightarrow \delta-\mathrm{ISOPO}+\mathrm{NO}_{2}$ pathway (Galloway et al., 2011; Peeters and Nguyen, 2012; Nguyen and Peeters, 2015) becomes negligible under ambient atmospheric conditions due to the unimolecular reactions of the $\delta$-ISOPO2 reactions $\left(\mathrm{O}_{2}\right.$-elimination leading to $\beta$-ISOPO2 radicals, and 1,6 $\mathrm{H}$-shift isomerization) resulting in very small $\delta$-ISOPO2 fractions and vanishing $\delta$-ISOPO formation in the atmosphere (Peeters et al., 2014; Teng et al., 2017).

Furthermore, the oxidation of isoprene hydroxyepoxides (IEPOX), which was believed to be a potentially significant glyoxal source (Bates et al., 2014; Li et al., 2016), is found to produce very little glyoxal in atmospheric conditions due to the proposed fast $1,4 \mathrm{H}$-shift in the peroxy radical
Table 7. Global sources of glyoxal in the model simulation.

\begin{tabular}{|c|c|c|}
\hline & $\operatorname{Tg}(\mathrm{C}) \mathrm{yr}^{-1}$ & $\operatorname{Tg}(\mathrm{GLY}) \mathrm{yr}^{-1}$ \\
\hline \multicolumn{3}{|l|}{ Direct emissions } \\
\hline Biomass burning & 1.58 & 3.8 \\
\hline \multicolumn{3}{|l|}{ Photochemical production } \\
\hline $\mathrm{C}_{2} \mathrm{H}_{2}+\mathrm{OH}$ & 2.39 & 5.8 \\
\hline Aromatics $+\mathrm{OH}$ & 3.78 & 9.1 \\
\hline Monoterpene oxidation & 3.67 & 8.9 \\
\hline GLYALD + OH & 4.69 & 11.3 \\
\hline $\mathrm{IEPOX}+\mathrm{OH}$ & 0.08 & 0.2 \\
\hline $\mathrm{OCHCH}_{2} \mathrm{OOH}+\mathrm{OH}$ & 0.38 & 0.9 \\
\hline HPALDs & 0.92 & 0.6 \\
\hline $\mathrm{ISOPOOH}+\mathrm{OH}$ & 0.89 & 2.2 \\
\hline $\mathrm{ISOP}+\mathrm{NO}_{3}$ & 0.09 & 0.2 \\
\hline Other pathways in isoprene oxidation & 1.13 & 2.7 \\
\hline \multicolumn{3}{|l|}{ Total source } \\
\hline Global & 19.6 & 47 \\
\hline
\end{tabular}

IEPOXBO2 $\left(\mathrm{HOCH}_{2} \mathrm{CH}(\mathrm{OH}) \mathrm{C}\left(\mathrm{CH}_{3}\right)(\mathrm{O} 2) \mathrm{CHO}\right)$ formed from IEPOX $+\mathrm{OH}$ (Wennberg et al., 2018), outcompeting its reactions with $\mathrm{NO}$ and $\mathrm{HO}_{2}$ (see Note N19). The 1,4 H-shift rate is very uncertain and could be overestimated, but even a factor of 10 reduction of the rate would imply a fairly small glyoxal production due to IEPOX $+\mathrm{OH}\left(0.6 \mathrm{Tg} \mathrm{C} \mathrm{yr}^{-1}\right)$.

Chan Miller et al. (2017) suggested that the DIHPCARPs from the $1,6 \mathrm{H}$-shift of $\delta$-ISOPO2 partly undergoes a 1,5 H-shift to a dihydroperoxy dicarbonyl (DHDC, e.g. $\left.\mathrm{OCHCH}(\mathrm{OOH}) \mathrm{C}\left(\mathrm{CH}_{3}\right)(\mathrm{OOH}) \mathrm{CHO}\right)$, which would quickly photolyse into an oxy radical decomposing to glyoxal and other products. However, the yield of DIHPCARPs from $\delta$ ISOPO2 isomerization is now estimated to be much lower than previously assumed; furthermore, even under the assumption that the $1,5 \mathrm{H}$-shift would be competitive, and although DHDC photolysis should indeed be very rapid, direct $\mathrm{OH}$ release (followed by decomposition of the resulting oxy 
radical) should be negligible (Liu et al., 2018), whereas the expected preferred dissociation pathway involves formyl radical release and subsequent formation of $\mathrm{OH}$ and a hydroperoxy dicarbonyl. The latter might form glyoxal upon further photolysis but at much lower yields than in the mechanism of Chan Miller et al. (2017).

Finally, due to the fast photolysis of hydroperoxyacetaldehyde (HPAC), the fraction of the formed HPAC reacting with $\mathrm{OH}$ is small $(23 \%)$, and only a fraction of it gives glyoxal (along with $\mathrm{OH}$ ).

There are still large uncertainties in the mechanism, however, and direct experimental constraints on the glyoxal yields in real atmospheric conditions are lacking. Further work is needed to refine the above estimates and identify additional sources, since model evaluations against spaceborne and in situ glyoxal measurements suggest a large photochemical source (Stavrakou et al., 2009b; Li et al., 2016; Silva et al., 2018).

\section{Conclusions}

We have presented a new BVOC oxidation mechanism for use in large-scale tropospheric chemistry-transport models. Its main focus is on isoprene, owing to its high chemical complexity and very large share of global BVOC emissions: of the 105 organic chemical species included in the mechanism, 97 compounds ( 74 stable compounds and 23 radicals) are involved in the chemical degradation of isoprene alone. This mechanism incorporates all major mechanistic advances from recent studies, in particular those affecting the budget of $\mathrm{HO}_{x}$ and $\mathrm{NO}_{x}$ radicals. Mainly thanks to $\mathrm{HO}_{x}$ formation in isomerization reactions of isoprene-derived peroxy radicals and further $\mathrm{OH}$ recycling through secondary reactions, the mechanism goes a long way in explaining the large underestimations of modelled $\mathrm{OH}$ concentrations in isoprene-rich, $\mathrm{NO}_{x}$-poor areas that prompted the community to search for OH-recycling mechanisms about a decade ago (Lelieveld et al., 2008; Hofzumahaus et al., 2009). The representation of monoterpene chemistry is much cruder, due to the still very poor understanding of its formidably complex mechanism. The simple monoterpene mechanism included here is only meant to provide an approximate reproduction of the yield of key OVOCs produced in their oxidation, based on box model simulations with the Master Chemical Mechanism (MCM).

Although smaller than, e.g. the Caltech mechanism or the MCMv3.3.1, this isoprene mechanism is larger than most mechanisms implemented in large-scale models and probably more detailed than strictly needed for many modelling purposes, such as the prediction of isoprene impacts on $\mathrm{HO}_{x}, \mathrm{NO}_{x}$, and ozone. Reduction techniques could be implemented to lighten the mechanism while retaining its most essential predictions, but since its current size and degree of detail can be handled by MAGRITTE, we find it useful to keep it as is in order to facilitate further analysis of model results and future mechanism updates. As pointed out by Wennberg et al. (2018), the distinction between isoprene peroxys resulting from $\mathrm{OH}$ addition to $\mathrm{C}_{1}$ and $\mathrm{C}_{4}$ is essential in view of the order of magnitude difference in bulk isomerization rates (Fig. 6) and in the difference in the nature of the resulting products. For example, the distinction also impacts the fate of the first-generation hydroxynitrates, given the efficient hydrolysis of the tertiary 1,2-isoprene hydroxynitrate. Note that the hydrolysis rates remain very uncertain. Due to our assumption of very fast tertiary nitrate hydrolysis $(\gamma=0.03)$, about $50 \%$ of the global sink of the 1,2-isoprene hydroxynitrate is due to this process. The rate might be too high, but it accounts for the fast overall hydroxynitrate loss observed in campaign measurements. This aspect of the mechanism will be revised when quantitative experimental determinations of heterogeneous processes and rates become available.

Although many parts of our isoprene mechanism rely on the Caltech mechanism, there are notable differences. Most importantly, the $1,6 \mathrm{H}$-shift of the $Z$ - $\delta$-hydroxyperoxy radicals generate HPALD at high yield $(75 \%$ vs. $25 \%$ in the Caltech mechanism), whereas the DIHPCARPs turn out to be minor compounds, undergoing $\mathrm{H}$-shift reactions along lines differing from previous work. This product distribution is fully consistent the recent experimental results of Berndt et al. (2019), supported and complemented by earlier theoretical results (Peeters and Nguyen, 2012; Peeters et al., 2014).

Another major difference between the present and previous isoprene mechanisms lies in the very fast photolysis of $\alpha$-hydroperoxycarbonyls (Liu et al., 2018), leading in several important cases to the formation of an enol, which is in large part oxidized by $\mathrm{OH}$ into formic or acetic acid. Also new to this mechanism, $\mathrm{HC}(\mathrm{O}) \mathrm{OH}$ is formed from the $\mathrm{OH}$ oxidation of keto-enols (HMVK and HMAC) produced from the photolysis of several multifunctional carbonyls. This pathway of HMVK/HMAC is all the more relevant as their photolysis is likely much slower than previously thought. More generally, the oxidation of enols formed from the oxidation of isoprene, acetaldehyde, and acetone by $\mathrm{OH}$ is a potentially large, previously unsuspected source of carboxylic acids estimated here at $9 \mathrm{Tg}(\mathrm{HC}(\mathrm{O}) \mathrm{OH}) \mathrm{yr}^{-1}$ (slightly larger than the contribution of alkene ozonolysis) and $11 \mathrm{Tg}\left(\mathrm{CH}_{3} \mathrm{C}(\mathrm{O}) \mathrm{OH}\right) \mathrm{yr}^{-1}$. This source amounts to a significant share $(\sim 28 \%$ for $\mathrm{HC}(\mathrm{O}) \mathrm{OH}$ and $15 \%$ for $\left.\mathrm{CH}_{3} \mathrm{C}(\mathrm{O}) \mathrm{OH}\right)$ of the total identified global source, which remains, however, largely insufficient to account for the atmospheric observations for both compounds (e.g., Paulot et al., 2011). Further experimental and theoretical studies of multifunctional carbonyl photolysis and enol oxidation are required to confirm and refine those estimates. The source could be larger due to the neglected contribution of hydroperoxycarbonyls formed from higher anthropogenic NMVOCs (e.g. higher ketones and their precursors) and possibly monoterpenes. Moreover, the contribution of acetaldehyde photooxidation could be much higher than estimated here, considering the large underestimation of its 
calculated concentrations at remote locations (Read et al., 2012).

Evaluation of MAGRITTE and of its new chemical mechanism against the SEAC ${ }^{4} \mathrm{RS}$ campaign measurements indicates a good overall model performance for the main isoprene oxidation products. Heterogeneous reactions of IEPOX and organic nitrates on aerosols are a large area of uncertainty, with suggestions of heterogeneous sink overestimation for tertiary organic nitrates and sink underestimations for other isoprene nitrates. The total $\mathrm{RONO}_{2}$ concentrations are underestimated by about $40 \%$, possibly due to misrepresentations of nitrates from, e.g. monoterpenes and anthropogenic precursors. The low observed $\mathrm{CH}_{3} \mathrm{ONO}_{2}$ levels are well reproduced by the model, providing a strong indication of a very low nitrate yield $\left(<3 \times 10^{-4}\right)$ in the $\mathrm{CH}_{3} \mathrm{O}_{2}+\mathrm{NO}$ reaction.

Code and data availability. The chemical mechanism is available at https://doi.org/10.18758/71021042 in KPP (Kinetic PreProcessor) format (Müller and Peeters, 2018), including equation and species files, fortran code for calculating the reaction rates, and absorption cross section data files for polyfunctional carbonyls. Other relevant subroutines of the MAGRITTE model can be made available upon request (email: jeanfrancois.muller@aeronomie.be). The SEAC ${ }^{4} \mathrm{RS}$ airborne trace gas measurements are available from the NASA LaRC Airborne Science Data for Atmospheric Composition (https://www-air.larc.nasa. gov/missions/merges/, last access: 15 April 2019).

Author contributions. JFM and JP elaborated the mechanism and drafted the manuscript, JFM and TS conducted the model calculations, and all authors analysed the model results.

Competing interests. The authors declare that they have no conflict of interest.

Acknowledgements. We gratefully acknowledge the PIs and data managers of the NASA SEAC ${ }^{4} \mathrm{RS}$ campaign for the measurements used in this work.

Financial support. This research was supported by the Belgian Science Policy Office through the projects TROVA (2016-2018), within the ESA/PRODEX programme, OCTAVE (2017-2021), within the BRAIN-be research programme, and BIOSOA, within the SSD program (2006-2010).

Review statement. This paper was edited by David Topping and reviewed by three anonymous referees.

\section{References}

Allen, H. M., Crounse, J. D., Bates, K. H., Teng, A. P., KrawiecThayer, M. P., Rivera-Rios, J. C., Keutsch, F. N., St. Clair, J. M., Hanisco, T. F., Moller, K. H., Kjaergaard, H. G., and Wennberg, P. O.: Kinetics and product yields of the $\mathrm{OH}$ initiated oxidation of hydroxymethyl hydroperoxide, J. Phys. Chem. A, 122, 62926302, 2018.

Anderson, D. C., Loughner, C. P., Diskin, G., Weinheimer, A., Canty, T. P., Salawitch, R. J., Worden, H. M., Fried, A., Mikoviny, T., Wisthaler, A., and Dickerson, R. R.: Measured and modeled $\mathrm{CO}$ and $\mathrm{NO}_{y}$ in DISCOVER-AQ: An evaluation of emissions and chemistry over the eastern US, Atmos. Environ., 96, 78-87, 2014.

Andrews, D., Heazlewood, B. R., Maccarone, A. T., Conroy, T., Payne, R. J., Jordan, M. J. T., and Kable, S. H.: Phototautomerization of acetaldehyde to vinyl alcohol: A potential route to tropospheric acids, Science, 337, 1203-1208, 2012.

Archibald, A., R., M. M., Taatjes, C. A., Percival, C. J., and Shallcross, D. E.: Atmospheric transformation of enols: A potential secondary source of carboxylic acids in the urban troposphere, Geophys. Res. Lett., 34, L21801, https://doi.org/10.1029/2007GL031032, 2007.

Arey, J., Aschmann, , S. M., Kwok, E. S. C., and Atkinson, R.: Alkyl nitrate, hydroxyalkyl nitrate, and hydroxycarbonyl formation from the $\mathrm{NO}_{x}$-air photooxidations of $\mathrm{C}_{5}-\mathrm{C}_{8}$ n-alkanes, $\mathrm{J}$. Phys. Chem. A, 105, 1020-1027, 2001.

Arneth, A., Schurgers, G., Lathiere, J., Duhl, T., Beerling, D. J., Hewitt, C. N., Martin, M., and Guenther, A.: Global terrestrial isoprene emission models: sensitivity to variability in climate and vegetation, Atmos. Chem. Phys., 11, 8037-8052, https://doi.org/10.5194/acp-11-8037-2011, 2011.

Asatryan, R., da Silva, G., and Bozzelli, J. W.: Quantum chemical study of the acrolein $\left(\mathrm{CH}_{2} \mathrm{CHCHO}\right)+\mathrm{OH}+\mathrm{O}_{2}$ reactions, $\mathrm{J}$. Phys. Chem. A, 114, 8302-8311, 2010.

Assaf, E., Song, B., Tomas, A., Schoemaecker, C., and Fittschen, C.: The rate constant of the reaction between $\mathrm{CH}_{3} \mathrm{O}_{2}$ radicals and $\mathrm{OH}$ radicals revisited, J. Phys. Chem. A, 120, 8923-8932, 2016.

Assaf, E., Sheps, L., Whalley, L., Heard, D., Tomas, A., Schoemacker, C., and Fittschen, C.: The reaction between $\mathrm{CH}_{3} \mathrm{O}_{2}$ and $\mathrm{OH}$ radicals: Product yields and atmospheric implications, Environ. Sci Technol., 51, 2170-2177, 2017.

Assaf, E., Schoemacker, C., Vereecken, L., and Fittschen, C.: Experimental and theoretical investigation of the reaction of $\mathrm{RO}_{2}$ radicals with $\mathrm{OH}$ radicals: Dependence of the $\mathrm{HO}_{2}$ yield on the size of the alkyl group, Int. J. Chem. Kinet., 1-11, 2018.

Atkinson, R., Baulch, D. L., Cox, R. A., Crowley, J. N., Hampson, R. F., Hynes, R. G., Jenkin, M. E., Rossi, M. J., Troe, J., and IUPAC Subcommittee: Evaluated kinetic and photochemical data for atmospheric chemistry: Volume II - gas phase reactions of organic species, Atmos. Chem. Phys., 6, 3625-4055, https://doi.org/10.5194/acp-6-3625-2006, 2006.

Back, R. A. and Yamamoto, S.: The gas-phase photochemistry and thermal decomposition of glyoxylic acid, Can. J. Chem., 63, 542-548, 1985.

Baeza-Romero, M. T., Glowacki, D. R., Blitz, M. A., Heard, D. E., Pilling, M. J., Rickard, A. R., and Seakins, P. W.: A combined experimental and theoretical study of the reaction between methyl- 
glyoxal and OH/OD radical: $\mathrm{OH}$ regeneration, Phys. Chem. Chem. Phys., 9, 4114-4128, 2007.

Barnes, I., Becker, K. H., and Zhu, T.: Near UV absorption spectra and photolysis products of difunctional organic nitrates: Possible importance as $\mathrm{NO}_{x}$ reservoirs, J. Atmos. Chem., 17, 353-373, 1993.

Bates, K. H., Crounse, J. D., St Clair, J. M., Bennet, N. B., Nguyen, T. B., Seinfeld, J. H., Stoltz, B. M., and Wennberg, P. O.: Gas phase production and loss of isoprene epoxydiols, J. Phys. Chem. A, 118, 1237-1246, 2014.

Bates, K. H., Nguyen, T. B., Teng, A. P., Crounse, J. D., Kjaergaard, H. G., Stoltz, B. M., Seinfeld, J. H., and Wennberg, P. O.: Production and fate of $\mathrm{C}_{4}$ dihydroxycarbonyl compounds from isoprene oxidation, J. Phys. Chem. A, 120, 106-117, 2016.

Baulch, D. L., Bowman, C. T., Cobos, C. J., Cox, R. A., Just, T., Kerr, J. A., Pilling, M. J., Stocker, D., Troe, J., Tsang, W., Welker, R. W., and Warnatz, J.: Evaluated kinetic data for combustion modeling: Supplement II, J. Phys. Chem. Ref. Data, 34, 7571397, 2005.

Bauwens, M., Stavrakou, T., Müller, J.-F., De Smedt, I., Van Roozendael, M., van der Werf, G. R., Wiedinmyer, C., Kaiser, J. W., Sindelarova, K., and Guenther, A.: Nine years of global hydrocarbon emissions based on source inversion of OMI formaldehyde observations, Atmos. Chem. Phys., 16, 1013310158, https://doi.org/10.5194/acp-16-10133-2016, 2016.

Bauwens, M., Stavrakou, T., Müller, J.-F., Van Schaeybroeck, B., De Cruz, L., De Troch, R., Giot, O., Hamdi, R., Termonia, P., Laffineur, Q., Amelynck, C., Schoon, N., Heinesch, B., Holst, T., Arneth, A., Ceulemans, R., Sanchez-Lorenzo, A., and Guenther, A.: Recent past (1979-2014) and future (2070-2099) isoprene fluxes over Europe simulated with the MEGAN-MOHYCAN model, Biogeosciences, 15, 3673-3690, https://doi.org/10.5194/bg-15-3673-2018, 2018.

Berndt, T., Jokinen, T., Sipilä, M., Mauldin III, R. L., Herrmann, H., Stratmann, F., Junninen, H., and Kulmala, M.: $\mathrm{H}_{2} \mathrm{SO}_{4}$ formation from the gas-phase reaction of stabilized Criegee In ediate with $\mathrm{SO}_{2}$ : Influence of water vapour content and temperature, Atmos. Environ., 89, 603-612, 2014.

Berndt, T., Huttinen, N., Herrmann, H., and Hansel, A.: First oxidation products from the reaction of hydroxyl radicals with isoprene for pristine environmental conditions, Commun. Chem., 2, 21, https://doi.org/10.1038/s42004-019-0120-9, 2019.

Bloss, C., Wagner, V., Jenkin, M. E., Volkamer, R., Bloss, W. J., Lee, J. D., Heard, D. E., Wirtz, K., Martin-Reviejo, M., Rea, G., Wenger, J. C., and Pilling, M. J.: Development of a detailed chemical mechanism (MCMv3.1) for the atmospheric oxidation of aromatic hydrocarbons, Atmos. Chem. Phys., 5, 641-664, https://doi.org/10.5194/acp-5-641-2005, 2005.

Bossolasco, A., Farago, E. P., Schoemaker, C., and Fittschen, C.: Rate constant of the reaction between $\mathrm{CH}_{3} \mathrm{O}_{2}$ and $\mathrm{OH}$ radicals, Chem. Phys. Lett., 593, 7-13, 2014.

Bottorff, B., Lew, M., Rickly, P., and Stevens, P. S.: HO $\mathrm{H}_{x}$ radical chemistry in a forested environment during PROPHET-AMOS 2016: Model comparisons and radical budget, AGU (American Geophysical Union) Fall Meeting, 10-14 December 2018, Washington D. C., USA, 2018.

Browne, E. C., Min, K.-E., Wooldridge, P. J., Apel, E., Blake, D. R., Brune, W. H., Cantrell, C. A., Cubison, M. J., Diskin, G. S., Jimenez, J. L., Weinheimer, A. J., Wennberg, P. O., Wisthaler, A., and Cohen, R. C.: Observations of total $\mathrm{RONO}_{2}$ over the boreal forest: $\mathrm{NO}_{x}$ sinks and $\mathrm{HNO}_{3}$ sources, Atmos. Chem. Phys., 13, 4543-4562, https://doi.org/10.5194/acp-13-4543-2013, 2013.

Browne, E. C., Wooldridge, P. J., Min, K.-E., and Cohen, R. C.: On the role of monoterpene chemistry in the remote continental boundary layer, Atmos. Chem. Phys., 14, 1225-1238, https://doi.org/10.5194/acp-14-1225-2014, 2014.

Burkholder, J. B., Sander, S. P., Abbatt, J. P. D., Barker, J. R., Huie, R. E., Kolb, C. E., Kurylo, M. J., Orkin, V. L., Wilmouth, D. M., and Wine, P. H.: Chemical Kinetics and Photochemical Data for Use in Atmospheric Studies, Evaluation number 18. JPL Publication 15-10, Jet Propulsion Laboratory, Pasadena, USA, 2015.

Butkovskaya, N. I., Pouvesle, N., Kukui, A., Mu, Y., and Le Bras, G.: Mechanism of the $\mathrm{OH}$-initiated oxidation of hydroxyacetone over the temperature range 236-298 K, J. Phys Chem. A, 110, 6833-6843, 2006a.

Butkovskaya, N. I., Pouvesle, N., Kukui, A., and Bras, G. L.: Mechanism of the $\mathrm{OH}$-initiated oxidation of glycolaldehyde over the temperature Range 233-296 K, J. Phys. Chem., 110, 13492 13499, 2006b.

Butkovskaya, N. I., Kukui, A., and Le Bras, G.: Pressure and temperature dependence of methyl nitrate formation in the $\mathrm{CH}_{3} \mathrm{O}_{2}+$ NO Reaction, J. Phys. Chem. A, 116, 5972-5980, 2012.

Cady-Pereira, K. E., Chaliyakunnel, S., Shephard, M. W., Millet, D. B., Luo, M., and Wells, K. C.: HCOOH measurements from space: TES retrieval algorithm and observed global distribution, Atmos. Meas. Tech., 7, 2297-2311, https://doi.org/10.5194/amt7-2297-2014, 2014.

Calvert, J. G., Mellouki, A., Orlando, J., Pilling, M., and Wallington, T.: The mechanisms of atmospheric oxidation of the oxygenates, Oxford University Press, New York, USA, 1619 pp., 2011.

Capouet, M., Peeters, J., Nozière, B., and Müller, J.-F.: Alphapinene oxidation by $\mathrm{OH}$ : simulations of laboratory experiments, Atmos. Chem. Phys., 4, 2285-2311, https://doi.org/10.5194/acp4-2285-2004, 2004.

Caravan, R. L., Khan, M. A. H., Z'ador, J., Sheps, L., Antonov, I. O., Rotavera, B., Ramasesha K. Au, K., Chen, M.-W., Rösch, D., Osborn, D. L., Fittschen, C., Schoemaecker, C., Duncianu, M., Frira, A., Dusanter, S., Tomas, A., Percival, C. J., Shallcross, D. E., and Taatjes, C. A.: The reaction of hydroxyl and methylperoxy radicals is not a major source of atmospheric methanol, Nat. Commun., 9, 4343, https://doi.org/10.1038/s41467-018-06716$\mathrm{x}, 2018$.

Carlton, A. G., de Gouw, J., Jimenez, J. L., Ambrose, J. L., Attwood, A. R., Brown, S., Baker, K. R., Cohen, R. C., Edgerton, S., Farkas, C. M., Farmer, D., Goldstein, A. H., Gratz, L., Guenther, A., Hunt, S., Jaeglé, L., Jaffe, D. A., Mak, J., McClure, C., Nenes, A., Nguyen, T. K., Pierce, J. R., de Sa, S., Selin, N. E., Shah, V., Shaw, S., Shepson, P. B., Song, S., Stutz, J., Surratt, J. D., Turpin, B. J., Warneke, C., Washenfelder, R. A., Wennberg, P. O., and Zhou, X.: Synthesis of the Southeast Atmosphere Studies: Investigating fundamental atmospheric chemistry questions, B. Am. Meterol. Soc., 99, 547-567, 2018.

Carter, W. P. L. and Atkinson, R.: Alkyl nitrate formation from the atmospheric photooxidation of alkanes; a revised estimation method, J. Atmos. Chem., 8, 165-173, 1989.

Cazorla, M., Wolfe, G. M., Bailey, S. A., Swanson, A. K., Arkinson, H. L., and Hanisco, T. F.: A new airborne laser-induced fluores- 
cence instrument for in situ detection of formaldehyde throughout the troposphere and lower stratosphere, Atmos. Meas. Tech., 8, 541-552, https://doi.org/10.5194/amt-8-541-2015, 2015.

Chan, A. W. H., Galloway, M. M., Kwan, A. J., Chhabra, P. S., Keutsch, F. N., Wennberg, P. O., Flagan, R. C., and Seinfeld, J. H.: Photooxidation of 2-methyl-3-buten-2-ol (MBO) as a potential source of Secondary Organic Aerosol, Environ. Sci. Technol., 43, 4647-4652, 2009.

Chan Miller, C., Jacob, D. J., González Abad, G., and Chance, K.: Hotspot of glyoxal over the Pearl River delta seen from the OMI satellite instrument: implications for emissions of aromatic hydrocarbons, Atmos. Chem. Phys., 16, 4631-4639, https://doi.org/10.5194/acp-16-4631-2016, 2016.

Chan Miller, C., Jacob, D. J., Marais, E. A., Yu, K., Travis, K. R., Kim, P. S., Fisher, J. A., Zhu, L., Wolfe, G. M., Hanisco, T. F., Keutsch, F. N., Kaiser, J., Min, K.-E., Brown, S. S., Washenfelder, R. A., González Abad, G., and Chance, K.: Glyoxal yield from isoprene oxidation and relation to formaldehyde: chemical mechanism, constraints from SENEX aircraft observations, and interpretation of OMI satellite data, Atmos. Chem. Phys., 17, 8725-8738, https://doi.org/10.5194/acp-17-8725-2017, 2017.

Chao, W., Hsieh, J.-T., Chang, C.-H., and Lin, J. J.-M.: Direct kinetic measurement of the reaction of the simplest Criegee intermediate with water vapor, Science, 347, 751-754, 2015.

Claeys, M., Graham, B., Vas, G., Wang, W., Vermeylen, R., Pashynska, V., Cafmeyer, J., Guyon, P., Andreae, M. O., Artaxo, P., and Maenhaut, W.: Formation of secondary organic aerosols through photooxidation of isoprene, Science 303, 1173-1176, 2004.

Compernolle, S., Ceulemans, K., and Müller, J.-F.: EVAPORATION: a new vapour pressure estimation methodfor organic molecules including non-additivity and intramolecular interactions, Atmos. Chem. Phys., 11, 9431-9450, https://doi.org/10.5194/acp-11-9431-2011, 2011.

Coote, M. L., Collins, M. A., and Radom, L.: Calculation of accurate imaginary frequencies and tunneling coefficients for hydrogen abstraction reactions using IRCmax, Mol. Phys., 101, 13291338, 2003.

Crounse, J. D., Paulot, F., Kjaergaard, H. G., and Wennberg, P. O.: Peroxy radical isomerization in the oxidation of isoprene, Phys. Chem. Chem. Phys., 13, 13607-13613, Amendment: http: //www.rsc.org/suppdata/cp/c1/c1cp21330j/addition.htm (last access: 4 June 2019), 2011.

Crounse, J. D., Knap, H. C., Ørnsø, K. B., Jørgensen, S., Paulot, F., Kjaergaard, H. G., and Wennberg, P. O.: Atmospheric fate of methacrolein. 1. Peroxy radical isomerization following addition of $\mathrm{OH}$ and $\mathrm{O}_{2}$, J. Phys. Chem. A, 116, 5756-5762, 2012.

Crounse, J. D., Nielsen, L. B., Jørgensen, S., Kjaergaard, H. K., and Wennberg, P. O.: Autoxidation of organic compounds in the atmosphere, J. Phys. Chem. Lett., 4, 3513-3520, 2013.

D’Ambro, E. L., Møller, K. H., Lopez-Hilfiker, F. D., Schobesberger, S., Liu, J., Shilling, J. E., Kjaergaard, H. G., and Thornton, J. A.: Isomerization of second-generation isoprene peroxy radicals: Epoxide formation and implications for Secondary Organic Aerosol yields, Environ. Sci. Technol., 51, 4978-7987, 2017.

D’Ambro, E. L., Schobesberger, S., Gaston, C. J., Lopez-Hilfiker, F. D., Lee, B. H., Liu, J., Zelenyuk, A., Bell, D., Cappa, C. D., Helgestad, T., Li, Z., Guenther, A., Wang, J., Wise, M., Caylor, R., Surratt, J. D., Riedel, T., Hyttinen, N., Salo, V.-
T., Hasan, G., Kurtén, T., Shilling, J. E., and Thornton, J. A.: Chamber-based insights into the factors controlling IEPOX SOA yield, composition, and volatility, Atmos. Chem. Phys. Discuss., https://doi.org/10.5194/acp-2019-271, in review, 2019.

Damian, V., Sandu, A., Damian, M., Potra, F., and Carmichael, G.: The Kinetic PreProcessor KPP - A software environment for solving chemical kinetics, Comput. Chem. Eng., 26, 1567-1579, 2002.

da Silva, G.: Carboxylic acid catalyzed keto-enol tautomerizations in the gas phase, Angew. Chem., 122, 7685-7687, 2010.

Dee, D. P., Uppala, Simmons, A. J., Berrisford, P., Poli, P., Kobayashi, S., Andae, U., Balmaseda, M. A., Balsamo, G., Bauer, P., Bechtold, P., Beljaars, A. C. M., van de Berg, L., Bidlot, J., Bormann, N., Delsol, C., Dragani, R., Fuentes, M., Geer, A. J., Haimberger, L., Healy, S. B., Hersbach, H., Hólm, E. V., Isaksen, L., K P., Köhler, M., Matricardi, M., McNally, A. P., Monge-Sanz, B. M., Morcrette, J.-J., Park, B.-K., Peubey, C., de Rosnay, P., Tavolato, C., Thépaut, J.-N., and Vitart, F.: The ERA-Interim reanalysis: configuration and performance of the data assimilation system, Q. J. Roy. Meteor. Soc, 137, 553-597, https://doi.org/10.1002/qj.828, 2011.

Dibble, T. S.: Intramolecular hydrogen bonding and double H-Atom transfer in peroxy and alkoxy radicals from isoprene, J. Phys. Chem. A, 108, 2199-2207, 2004.

Fischer, E. V., Jacob, D. J., Millet, D. B., Yantosca, R. M., and Mao, J.: The role of the ocean in the global atmospheric budget of acetone, Geophys. Res. Lett., 39, L01807, https://doi.org/10.1029/2011GL050086, 2012.

Fisher, J. A., Jacob, D. J., Travis, K. R., Kim, P. S., Marais, E. A., Chan Miller, C., Yu, K., Zhu, L., Yantosca, R. M., Sulprizio, M. P., Mao, J., Wennberg, P. O., Crounse, J. D., Teng, A. P., Nguyen, T. B., St. Clair, J. M., Cohen, R. C., Romer, P., Nault, B. A., Wooldridge, P. J., Jimenez, J. L., CampuzanoJost, P., Day, D. A., Hu, W., Shepson, P. B., Xiong, F., Blake, D. R., Goldstein, A. H., Misztal, P. K., Hanisco, T. F., Wolfe, G. M., Ryerson, T. B., Wisthaler, A., and Mikoviny, T.: Organic nitrate chemistry and its implications for nitrogen budgets in an isoprene- and monoterpene-rich atmosphere: constraints from aircraft (SEAC $\left.{ }^{4} \mathrm{RS}\right)$ and ground-based (SOAS) observations in the Southeast US, Atmos. Chem. Phys., 16, 5969-5991, https://doi.org/10.5194/acp-16-5969-2016, 2016.

Flocke, F., Atlas, E., Madronich, S., Schauffler, S. M., Aikin, K., Margitan, J. J., and Bui, T. P.: Observations of methyl nitrate in the lower stratosphere during STRAT: Implications for its gas phase production mechanisms, Geophys. Res. Lett., 25, 18911894, 1998.

Fu, T.-M., Jacob, D. J., Wittrock, F., Burrows, J. P., Vrekoussis, M., and Henze, D. K.: Global budgets of atmospheric glyoxal and methylglyoxal, and implications for formation of secondary organic aerosols, J. Geophys. Res., 113, D15303, https://doi.org/10.1029/2007JD009505, 2008.

Fuchs, H., Hofzumahaus, A., Rohrer, F., Brauers, T., Dorn, H.-P., Häseler, R., Holland, F., Kaminski, M., Li, X., Lu, K., Nehr, S., Tillmann, R., Wegener, R., and Wahner, A.: Experimental evidence for efficient hydroxyl radical regeneration in isoprene oxidation, Nat. Geosci., 6, 1023-1026, 2013.

Galloway, M. M., Huisman, A. J., Yee, L. D., Chan, A. W. H., Loza, C. L., Seinfeld, J. H., and Keutsch, F. N.: Yields of oxidized volatile organic compounds during the $\mathrm{OH}$ radical initiated oxi- 
dation of isoprene, methyl vinyl ketone, and methacrolein under high-NO ${ }_{x}$ conditions, Atmos. Chem. Phys., 11, 10779-10790, https://doi.org/10.5194/acp-11-10779-2011, 2011.

Gross, C. B. M., Dillon, T. J., Schuster, G., Lelieveld, J., and Crowley, J. N.: Direct kinetic study of $\mathrm{OH}$ and $\mathrm{O}_{3}$ formation in the reaction of $\mathrm{CH}_{3} \mathrm{C}(\mathrm{O}) \mathrm{O}_{2}$ with $\mathrm{HO}_{2}$, J. Phys. Chem. A, 118, 974 $985,2014$.

Guenther, A. B., Jiang, X., Heald, C. L., Sakulyanontvittaya, T., Duhl, T., Emmons, L. K., and Wang, X.: The Model of Emissions of Gases and Aerosols from Nature version 2.1 (MEGAN2.1): an extended and updated framework for modeling biogenic emissions, Geosci. Model Dev., 5, 1471-1492, https://doi.org/10.5194/gmd-5-1471-2012, 2012.

Hallquist, M., Wenger, J. C., Baltensperger, U., Rudich, Y., Simpson, D., Claeys, M., Dommen, J., Donahue, N. M., George, C., Goldstein, A. H., Hamilton, J. F., Herrmann, H., Hoffmann, T., Iinuma, Y., Jang, M., Jenkin, M. E., Jimenez, J. L., Kiendler-Scharr, A., Maenhaut, W., McFiggans, G., Mentel, Th. F., Monod, A., Prévôt, A. S. H., Seinfeld, J. H., Surratt, J. D., Szmigielski, R., and Wildt, J.: The formation, properties and impact of secondary organic aerosol: current and emerging issues, Atmos. Chem. Phys., 9, 5155-5236, https://doi.org/10.5194/acp9-5155-2009, 2009.

Hermans, I., Muller, J.-F., Nguyen, T., Jacobs, P., and Peeters, J.: Kinetics of $\alpha$-hydroxy-alkylperoxyl radicals in oxidation processes. $\mathrm{HO}_{2}$-Initiated oxidation of ketones/aldehydes near the tropopause, J. Phys. Chem. A, 109, 4303-4311, 2005.

Hofzumahaus, A., Rohrer, F., Lu, K., Bohn, B., Brauers, T., Chang, C.-C., Fuchs, H., Holland, F., Kita, K., Kondo, Y., Li, X., Lou, S., Shao, M., Zeng, L., Wahner, A., and Zhang, Y.: Amplified trace gas removal in the troposphere, Science, 324, 1702-1704, 2009.

Huang, G., Brook, R., Crippa, M., Janssens-Maenhout, G., Schieberle, C., Dore, C., Guizzardi, D., Muntean, M., Schaaf, E., and Friedrich, R.: Speciation of anthropogenic emissions of non-methane volatile organic compounds: a global gridded data set for 1970-2012, Atmos. Chem. Phys., 17, 7683-7701, https://doi.org/10.5194/acp-17-7683-2017, 2017.

Jacobs, M. I., Burke, W. J., and Elrod, M. J.: Kinetics of the reactions of isoprene-derived hydroxynitrates: gas phase epoxide formation and solution phase hydrolysis, Atmos. Chem. Phys., 14, 8933-8946, https://doi.org/10.5194/acp-14-8933-2014, 2014.

Janssens-Maenhout, G., Crippa, M., Guizzardi, D., Dentener, F., Muntean, M., Pouliot, G., Keating, T., Zhang, Q., Kurokawa, J., Wankmüller, R., Denier van der Gon, H., Kuenen, J. J. P., Klimont, Z., Frost, G., Darras, S., Koffi, B., and Li, M.: HTAP_v2.2: a mosaic of regional and global emission grid maps for 2008 and 2010 to study hemispheric transport of air pollution, Atmos. Chem. Phys., 15, 11411-11432, https://doi.org/10.5194/acp-15-11411-2015, 2015.

Jenkin, M. E., Boyd, A. A., and Lesclaux, R.: Peroxy radical kinetics resulting from the $\mathrm{OH}$-initiated oxxidation of 1,3-butadiene, 2,3-dimethyl-1,3-butadiene and isoprene, J. Atmos. Chem., 29, 267-298, 1998.

Jenkin, M. E., Young, J. C., and Rickard, A. R.: The MCM v3.3.1 degradation scheme for isoprene, Atmos. Chem. Phys., 15, 11433-11459, https://doi.org/10.5194/acp-15-11433-2015, 2015.

Jokinen, T., Sipilä, M., Richters, S., Kerminen, V.-M., Paasonen, P., Stratmann, F., Worsnop, D., Kulmala, M., Ehn, M., Herrmann,
H., and Berndt, T.: Rapid autoxidation forms highly oxidized $\mathrm{RO}_{2}$ radicals in the atmosphere, Angew. Chem. Int. Ed., 53, 1-6, 2014.

Jokinen, T., Berndt, T., Makkonen, R., Kerminen, V.-M., Junninen, H., Paasonen, P., Stratmann, F., Herrmann, H., Guenther, A. B., Worsnop, D. R., Kulmala, M., Ehn, M., and Sipilä, M.: Production of extremely low volatile organic compounds from biogenic emissions: Measured yields and atmospheric implications, P. Nat. Acad. Sci. USA, 112, 7123-7128, 2015.

Jorand, F., Kerhoas, L., Heiss, A., Einhorn, J., and Sahetchian, K.: Determination of the ultraviolet absorption cross section of hexyl-ketohydroperoxides in solution in acetonitrile, J. Photochem. Photobiol. A, 134, 119-125, 2000.

Jørgensen, S., Knap, H. C., Otkjaer, R. V., Jensen, A. M., Kjeldsen, M. L. H., Wennberg, P. O., and Kjaergaard, H. G.: Rapid hydrogen shift scrambling in hydroperoxy-substituted organic peroxy radicals, J. Phys. Chem A, 120, 266-275, 2016.

Khan, M. A. H., Lyons, K., Chhantyal-Pun, R., McGillen, M. R., Caravan, R. L., Taatjes, C. A., Orr-Ewing, A. J., Percival, C. J., and Shallcross, D. E.: Investigating the tropospheric chemistry of acetic acid using the global 3-D chemistry transport model, STOCHEM-CRI, J. Geophys., 123, 6267-6281, 2018.

Kjaergaard, H. G., Knap, H. C., Ørnsø, K. B., Jørgensen, S., Crounse, J. D., Paulot, F., and Wennberg, P. O.: Atmospheric fate of methacrolein. 2. Formation of lactone and implications for organic aerosol production, J. Phys. Chem. A, 116, 5763-5768, 2012.

Knap, H. C., Schmidt, J. A., and Jorgensen, S.: Hydrogen shift reactions in four methyl-buten-ol (MBO) peroxy radicals and their impact on the atmosphere, Atmos. Environ., 147, 79-87, 2016.

Kwan, A. J., Chan, A. W. H., Ng, N. L., Kjaergaard, H. G., Seinfeld, J. H., and Wennberg, P. O.: Peroxy radical chemistry and $\mathrm{OH}$ radical production during the $\mathrm{NO}_{3}$-initiated oxidation of isoprene, Atmos. Chem. Phys., 12, 7499-7515, https://doi.org/10.5194/acp-12-7499-2012, 2012.

Lee, L., Teng, A. P., Wennberg, P. O., Crounse, J. D., and Cohen, R. C.: On rates and mechanisms of $\mathrm{OH}$ and $\mathrm{O}_{3}$ reactions with isoprene-derived hydroxy nitrates, J. Phys. Chem. A, 118, 16221637, 2014.

Lei, X., Chen, D., Wang, W., Liu, F., and Wang, W.: Quantum chemical studies of the $\mathrm{OH}$-initiated oxidation reactions of propenols in the presence of $\mathrm{O}_{2}$, Mol. Phys., 117, 682-692, https://doi.org/10.1080/00268976.2018.1537527, 2018.

Lelieveld, J., Butler, T. M., Crowley, J. N., Dillon, T. J., Fischer, H., Ganzeveld, L., Harder, H., Lawrence, M. G., Martinez, M., Taraborrelli, D., and Williams, J.: Atmospheric oxidation capacity sustained by a tropical forest, Nature, 452, 737-740, 2008.

Lelieveld, J., Gromov, S., Pozzer, A., and Taraborrelli, D.: Global tropospheric hydroxyl distribution, budget and reactivity, Atmos. Chem. Phys., 16, 12477-12493, https://doi.org/10.5194/acp-1612477-2016, 2016.

Lewis, T. R., Blitz, M. A., Heard, D. E., and Seakins, P. W.: Direct evidence for a substantive reaction between the Criegee intermediate, $\mathrm{CH}_{2} \mathrm{OO}$, and the water vapour dimer, Phys. Chem. Chem. Phys., 17, 4859-4863, 2015.

Li, J., Mao, J., Min, K.-E., Washenfelder, R., Brown, S. S., Kaiser, J., Keutsch, F. N., Volkamer, R., Wolfe, G. M., Hanisco, T. F., Pollack, I. B., Ryerson, T. B., Graus, M., Gilman, J. B., Lerner, B. M., Warneke, C., de Gouw, J. A., Middlebrook, A. 
M., Liao, J., Welti, A., Henderson, B. H., McNeill, V. F., Hall, S. R., Ullmann, K., Donner, L. J., Paulot, F., and Horowitz, L. W.: Observational constraints on glyoxal production from isoprene oxidation and its contribution to organic aerosol over the Southeast United States, J. Geophys. Res., 121, 9849-9861, https://doi.org/10.1002/2016JD025331, 2016.

Li, J., Mao, J., Fiore, A. M., Cohen, R. C., Crounse, J. D., Teng, A. P., Wennberg, P. O., Lee, B. H., Lopez-Hilfiker, F. D., Thornton, J. A., Peischl, J., Pollack, I. B., Ryerson, T. B., Veres, P., Roberts, J. M., Neuman, J. A., Nowak, J. B., Wolfe, G. M., Hanisco, T. F., Fried, A., Singh, H. B., Dibb, J., Paulot, F., and Horowitz, L. W.: Decadal changes in summertime reactive oxidized nitrogen and surface ozone over the Southeast United States, Atmos. Chem. Phys., 18, 2341-2361, https://doi.org/10.5194/acp18-2341-2018, 2018.

Liggio, J., Li, S.-M., and McLaren, R.: Reactive uptake of glyoxal by particulate matter, J. Geophys. Res., 110, D10304, https://doi.org/10.1029/2004JD005113, 2005.

Lin, Y.-H., Zhang, Z., Docherty, K. S., Zhang, H., Budisulistiorini, S. H., Rubitschun, C. L., Shaw, S. L., Knipping, E. M., Edgerton, E. S., Kleindienst, T. E., Gold, A., and Surratt, J. D.: Isoprene epoxydiols as precursors to secondary organic aerosol formation: acid-catalyzed reactive uptake studies with authentic compounds, Environ. Sci., 46, 250-258, 2012.

Lin, Y.-H., Zhang, H., Pye, H. O. T., Zhang, Z., Marth, W. J., Park, S., Arashiro, M., Cui, T., Budisulistiorini, S. H., Sexton, K. G., Vizuete, W., Xie, Y., Luecken, D. J., Piletic, I. R., Edney, E. O., Bartolotti, L. J., Glod, A., and Surratt, J. D.: Epoxide as a precursor to secondary organic aerosol formation from isoprene photooxidation in the presence of nitrogen oxides, P. Nat. Acad. Sci. USA, 110, 6718-6723, 2013.

Liu, J. M., D’Ambro, E., Lee, B. H., Lopez-Hilfiker, F. D., Zaveri, R. A., Rivera-Rios, J. C., Keutsch, F. N., Iyer, S., Kurten, T., Zhang, Z. F., Gold, A., Surratt, J. D., Shilling, J. E., and Thornton, J. A.: Efficient isoprene secondary organic aerosol formation from a non-IEPOX pathway, Environ. Sci Technol., 50, 9872 9880, 2016.

Liu, Y. J., Herdlinger-Blatt, I., McKinney, K. A., and Martin, S. T.: Production of methyl vinyl ketone and methacrolein via the hydroperoxyl pathway of isoprene oxidation, Atmos. Chem. Phys., 13, 5715-5730, https://doi.org/10.5194/acp-135715-2013, 2013.

Liu, Z., Nguyen, V. S., Harvey, J., Müller, J.-F., and Peeters, J.: Theoretically derived mechanisms of HPALD photolysis in isoprene oxidation, Phys. Chem. Chem. Phys., 19, 9096-9106, 2017.

Liu, Z., Nguyen, V. S., Harvey, J., Müller, J.-F., and Peeters, J.: The photolysis of $\alpha$-hydroperoxycarbonyls, Phys. Chem. Chem. Phys., 20, 6970-6979, 2018.

Madronich, S.: UV radiation in the natural and perturbed atmosphere, in Environmental Effects of Ultraviolet Radiation, edited by: Tevini, M., 17-69, Lewis, Boca Raton, Florida, USA, 1993.

Magneron, I., Mellouki, A., and Le Bras, G.: Photolysis and OHinitiated oxidation of glycolaldehyde under atmospheric conditions, J. Phys. Chem. A, 109, 4552-4561, 2005.

Malecha, K. T. and Niskodorov, S. A.: Photodegradation of secondary organic aerosol particles as a source of small, oxygenated volatile organic compounds, Environ. Sci. Technol., 50, 99909997, 2016.
Mao, J., Carlton, A., Cohen, R. C., Brune, W. H., Brown, S. S., Wolfe, G. M., Jimenez, J. L., Pye, H. O. T., Lee Ng, N., Xu, L., McNeill, V. F., Tsigaridis, K., McDonald, B. C., Warneke, C., Guenther, A., Alvarado, M. J., de Gouw, J., Mickley, L. J., Leibensperger, E. M., Mathur, R., Nolte, C. G., Portmann, R. W., Unger, N., Tosca, M., and Horowitz, L. W.: Southeast Atmosphere Studies: learning from model-observation syntheses, Atmos. Chem. Phys., 18, 2615-2651, https://doi.org/10.5194/acp18-2615-2018, 2018.

Marais, E. A., Jacob, D. J., Jimenez, J. L., Campuzano-Jost, P., Day, D. A., Hu, W., Krechmer, J., Zhu, L., Kim, P. S., Miller, C. C., Fisher, J. A., Travis, K., Yu, K., Hanisco, T. F., Wolfe, G. M., Arkinson, H. L., Pye, H. O. T., Froyd, K. D., Liao, J., and McNeill, V. F.: Aqueous-phase mechanism for secondary organic aerosol formation from isoprene: application to the southeast United States and co-benefit of $\mathrm{SO}_{2}$ emission controls, Atmos. Chem. Phys., 16, 1603-1618, https://doi.org/10.5194/acp16-1603-2016, 2016.

Mebel, A. M. and Kislov, V. V.: The $\mathrm{C}_{2} \mathrm{H}_{3}+\mathrm{O}_{2}$ reaction revisited: Is multireference treatment of the wave function really critical?, J. Phys. Chem. A, 109, 6993-6997, 2005.

Méreau, R., Rayez, M.-T., Rayez J.-C., Caralp, F., and Lesclaux, R.: Theoretical study on the atmospheric fate of carbonyl radicals: kinetics of decomposition reactions, Phys. Chem. Chem. Phys., 3, 4712-4717, 2001.

Millet, D. B., Guenther, A., Siegel, D. A., Nelson, N. B., Singh, H. B., de Gouw, J. A., Warneke, C., Williams, J., Eerdekens, G., Sinha, V., Karl, T., Flocke, F., Apel, E., Riemer, D. D., Palmer, P. I., and Barkley, M.: Global atmospheric budget of acetaldehyde: 3-D model analysis and constraints from in-situ and satellite observations, Atmos. Chem. Phys., 10, 3405-3425, https://doi.org/10.5194/acp-10-3405-2010, 2010.

Millet, D. B., Baasandorj, M., Farmer, D. K., Thornton, J. A., Baumann, K., Brophy, P., Chaliyakunnel, S., de Gouw, J. A., Graus, M., Hu, L., Koss, A., Lee, B. H., Lopez-Hilfiker, F. D., Neuman, J. A., Paulot, F., Peischl, J., Pollack, I. B., Ryerson, T. B., Warneke, C., Williams, B. J., and Xu, J.: A large and ubiquitous source of atmospheric formic acid, Atmos. Chem. Phys., 15, 6283-6304, https://doi.org/10.5194/acp-15-6283-2015, 2015.

Møller, K. H., Bates, K. H., and Kjaergaard, H. G.: The importance of peroxy radical hydrogen-shift reactions in atmospheric isoprene oxidation, J. Phys. Chem. A, 123, 920-930, 2019.

Müller, J.-F. and Brasseur, G.: IMAGES: A three-dimensional chemical transport model of the global troposphere, J. Geophys. Res., 100, 16445-16490, 1995.

Müller, J.-F. and Peeters, J.: MAGRITTE model code and data, https://doi.org/10.18758/71021042, 2018.

Müller, J.-F., Stavrakou, T., Wallens, S., De Smedt, I., Van Roozendael, M., Potosnak, M. J., Rinne, J., Munger, B., Goldstein, A., and Guenther, A. B.: Global isoprene emissions estimated using MEGAN, ECMWF analyses and a detailed canopy environment model, Atmos. Chem. Phys., 8, 1329-1341, https://doi.org/10.5194/acp-8-1329-2008, 2008.

Müller, J.-F., Peeters, J., and Stavrakou, T.: Fast photolysis of carbonyl nitrates from isoprene, Atmos. Chem. Phys., 14, $2497-$ 2508, https://doi.org/10.5194/acp-14-2497-2014, 2014.

Müller, J.-F., Liu, Z., Nguyen, V. S., Stavrakou, T., Harvey, J. N., and Peeters, J.: The reaction of methyl peroxy and hydroxyl rad- 
icals as a major source of atmospheric methanol, Nat. Commmun., 7, 13213, https://doi.org/10.1038/ncomms13213, 2016.

Müller, J.-F., Stavrakou, T., Bauwens, M., Compernolle, S., and Peeters, J.: Chemistry and deposition in the Model of Atmospheric composition at Global and Regional scales using Inversion Techniques for Trace gas Emissions (MAGRITTE v1.0). Part B. Dry deposition, Geosci. Model Dev. Discuss., https://doi.org/10.5194/gmd-2018-317, in review, 2018.

Nakanishi, H., Morita, H., and Nagakura, S.: Electronic structures and spectra of the keto and enol forms of acetylene, Bull. Chem. Soc. Jpn., 50, 2255-2261, 1977.

Neeb, P.: Structure-reactivity based estimation of the rate constants for hydroxyl radical reactions with hydrocarbons, J. Atmos. Chem., 35, 295-315, 2000.

Neu, J. L., Lawler, M. J., Prather, M. J., and Saltzmann, E. S.: Oceanic alkyl nitrates as a natural source of tropospheric ozone, Geophys. Res. Lett., 35, L13814, https://doi.org/10.1029/2008GL034189, 2008.

Nguyen, T. B., Bates, K. H., Crounse, J., Schwantes, R. H., Zhang, X., Kjaergaard, H. G., D., S. J., Lin, P., Laskin, A., H., S. J., and Wennberg, P. O.: Mechanism of the hydroxyl radical oxidation of methacryloyl peroxynitrate (MPAN) and its pathway toward secondary organic aerosol formation in the atmosphere, Phys. Chem. Chem. Phys., 17, 17914-17926, 2015 a.

Nguyen, T. B., Crounse, J. D., Teng, A. P., St Clair, J. M., Paulot, F., Wolfe, G. M., and Wennberg, P. O.: Rapid deposition of oxidized biogenic compounds to a temperate forest, P. Nat. Acad. Sci. USA, 112, E392-E401, 2015b.

Nguyen, T. B., Tyndall, G. S., Crounse, J. D., Teng, A. P., Bates, K. H., Schwantes, R. H., Coggon, M. M., Zhang, L., Feiner, P., Miller, D. O., Skog, K. M., Rivera-Rios, J. C., Dorris, M., Olson, K. F., Koss, A., Wild, R. J., Brown, S. B., Goldstein, A., de Gouw, J. A., Brune, B. H., Keutsch, F. N., Seinfeld, J. H., and Wennberg, P. O.: Atmospheric fates of Criegee intermediates in the ozonolysis of isoprene, Phys. Chem. Chem. Phys., 18, 10241-10254, 2016

Nguyen, V. S. and Peeters, J.: Fast (E) - (Z) Isomerization mechanisms of substituted allyloxy radicals in isoprene oxidation, J. Phys. Chem. A, 119, 7270-7276, 2015.

Novelli, A., Kaminski, M., Rolletter, M., Acir, I.-H., Bohn, B., Dorn, H.-P., Li, X., Lutz, A., Nehr, S., Rohrer, F., Tillmann, R., Wegener, R., Holland, F., Hofzumahaus, A., KiendlerScharr, A., Wahner, A., and Fuchs, H.: Evaluation of $\mathrm{OH}$ and $\mathrm{HO}_{2}$ concentrations and their budgets during photooxidation of 2-methyl-3-butene-2-ol (MBO) in the atmospheric simulation chamber SAPHIR, Atmos. Chem. Phys., 18, 11409-11422, https://doi.org/10.5194/acp-18-11409-2018, 2018a.

Novelli, A., Bohn, B., Dorn, H. P., Hofzumahaus, A., Holland, F., Li, X., Kaminski, M., Yu, Z., Rosanka, S., Reimer, D., Gkatzelis, G. I., Taraborrelli, D., Vereecken, L., Rohrer, F., Tillmann, R., Wegener, R., Kiendler-Scharr A., Wahner, A., and Fuchs, H.: The atmosphere of a tropical forest simulated in a chamber: experiments, theory and global significance of $\mathrm{OH}$ regeneration in isoprene oxidation, iCACGP-IGAC 2018 Conference, 2529 September 2018, Takamatsu, Japan, 2018b.

Novelli, A., Vereecken, L., Bohn, B., Dorn, H.-P., Hofzumahaus, A., Holland, F., Li, X., Kaminski, M., Yu, Z., Rosanka, S., Reimer, D., Gkatzelis, G. I., Taraborrelli, D., Rohrer, F., Tillmann, R., Wegener, R., Kiendler-Scharr, A., Wahner, A., and Fuchs, H.:
The impact of the aldehyde-hydrogen shift on the $\mathrm{OH}$ radical budget in the isoprene oxidation mechanism in pristine environments, Atmospheric CHemical Mechanism (ACM) Conference, 5-7 December 2018, Davis, USA, 2018c.

Orlando, J., Tyndall, G., and Taraborrelli, D.: Atmospheric oxidation mechanism for glycolaldehyde (and hydroxyacetone), $\mathrm{Ab}$ stract A33L-0315, AGU (American Geophysical Union) Fall Meeting, 3-7 December 2012, San Francisco, Calif., USA, 2012.

Paulot, F., Crounse, J. D., Kjaergaard, H. G., Kürten, A., St. Clair, J. M., Seinfeld, J. H., and Wennberg, P. O.: Unexpected epoxide formation in the gas-phase photooxidation of isoprene, Science, 325, 730-733, 2009a.

Paulot, F., Crounse, J. D., Kjaergaard, H. G., Kroll, J. H., Seinfeld, J. H., and Wennberg, P. O.: Isoprene photooxidation: new insights into the production of acids and organic nitrates, Atmos. Chem. Phys., 9, 1479-1501, https://doi.org/10.5194/acp-9-14792009, 2009b.

Paulot, F., Wunch, D., Crounse, J. D., Toon, G. C., Millet, D. B., DeCarlo, P. F., Vigouroux, C., Deutscher, N. M., González Abad, G., Notholt, J., Warneke, T., Hannigan, J. W., Warneke, C., de Gouw, J. A., Dunlea, E. J., De Mazière, M., Griffith, D. W. T., Bernath, P., Jimenez, J. L., and Wennberg, P. O.: Importance of secondary sources in the atmospheric budgets of formic and acetic acids, Atmos. Chem. Phys., 11, 1989-2013, https://doi.org/10.5194/acp-11-1989-2011, 2011.

Paulot, F., Henze, D. K., and Wennberg, P. O.: Impact of the isoprene photochemical cascade on tropical ozone, Atmos. Chem. Phys., 12, 1307-1325, https://doi.org/10.5194/acp-121307-2012, 2012.

Peeters, J. and Müller, J.-F.: $\mathrm{HO}_{x}$ radical regeneration in isoprene oxidation via peroxy radical isomerisations, II: Experimental evidence and global impact, Phys. Chem. Chem. Phys., 12, 14227 14235, 2010.

Peeters, J. and Nguyen, T. L.: Unusually fast 1,6-H shifts of enolic hydrogens in peroxy radicals: formation of the first-generation $\mathrm{C}_{2}$ and $\mathrm{C}_{3}$ carbonyls in the oxidation of isoprene, J. Phys. Chem. A, 116, 6134-6141, 2012.

Peeters, J., Fantechi, G., and Vereecken, L.: A generalized structureactivity relationship for the decomposition of (substituted) alkoxy radicals, J. Atmos. Chem., 48, 59-80, 2004.

Peeters, J., Nguyen, T. L., and Vereecken, L.: $\mathrm{HO}_{x}$ radical regeneration in the oxidation of isoprene, Phys. Chem. Chem. Phys., 11, 5935-5939, 2009.

Peeters, J., Müller, J.-F., Stavrakou, T., and Nguyen, S. V.: Hydroxyl radical recycling in isoprene oxidation driven by hydrogen bonding and hydrogen tunneling: the upgraded LIM1 mechanism, J. Phys. Chem. A, 118, 8625-8643, 2014.

Peeters, J., Nguyen, V. S., and Müller, J.-F.: Tautomerization of vinyl alcohol to acetaldehyde in the atmosphere revisited, J. Phys. Chem. Lett., 6, 4005-4011, 2015.

Pinho, P., Pio, C., and Jenkin, M.: Evaluation of isoprene degradation in the detailed tropospheric chemical mechanism, MCM v3, using environmental chamber data, Atmos. Environ., 39, 13031322, 2005.

Praske, E., Crounse, J. D., Bates, K. H., Kurten, T., Kjaergaard, H. G., and Wennberg, P. O.: Atmospheric fate of methyl vinyl ketone: peroxy radical reactions with $\mathrm{NO}$ and $\mathrm{HO}_{2}$, J. Phys. Chem. A, 119, 4562-4572, 2015. 
Praske, E., Otkjaer, R. V., Crounse, J. D., Hethcox, J. C., Stoltz, B. M., Kjaergaard, H. G., and Wennberg, P. O.: Atmospheric autoxidation is increasingly important in urban and suburban North America, P. Nat. Acad. Sci. USA, 115, 64-69, 2018.

Pugh, T. A. M., MacKenzie, A. R., Langford, B., Nemitz, E., Misztal, P. K., and Hewitt, C. N.: The influence of small-scale variations in isoprene concentrations on atmospheric chemistry over a tropical rainforest, Atmos. Chem. Phys., 11, 4121-4134, https://doi.org/10.5194/acp-11-4121-2011, 2011.

Read, K. A., Carpenter, L. J., Arnold, S. R., Beale, R., Nightingale, P. D., Hopkins, J. R., Lewis, A. C., Lee, J. D., Mendes, L., and Pickering, S. J.: Multiannual observations of acetone, methanol, and acetaldehyde in remote Tropical Atlantic air: Implications for atmospheric OVOC budgets and oxidative capacity, Envrion. Sci. Technol., 46, 11028-11039, 2012.

Richter, D., Weibring, P., Walega, J. G., Fried, A., Spuler, S. M., and Taubman, M. S.: Compact highly sensitive multi-species airborne mid-IR spectrometer, Appl. Phys. B, 119, 119-131, 2015.

Rindelaub, J. D., McAvey, K. M., and Shepson, P. B.: The photochemical production of organic nitrates from $\alpha$-pinene and loss via acid-dependent particle phase hydrolysis, Atmos. Environ., 100, 193-201, 2015.

Romer, P. S., Duffey, K. C., Wooldridge, P. J., Allen, H. M., Ayres, B. R., Brown, S. S., Brune, W. H., Crounse, J. D., de Gouw, J., Draper, D. C., Feiner, P. A., Fry, J. L., Goldstein, A. H., Koss, A., Misztal, P. K., Nguyen, T. B., Olson, K., Teng, A. P., Wennberg, P. O., Wild, R. J., Zhang, L., and Cohen, R. C.: The lifetime of nitrogen oxides in an isoprene-dominated forest, Atmos. Chem. Phys., 16, 7623-7637, https://doi.org/10.5194/acp16-7623-2016, 2016.

Röth, E.-P. and Ehhalt, D. H.: A simple formulation of the $\mathrm{CH}_{2} \mathrm{O}$ photolysis quantum yields, Atmos. Chem. Phys., 15, 7195-7202, https://doi.org/10.5194/acp-15-7195-2015, 2015.

Saunders, S. M., Jenkin, M. E., Derwent, R. G., and Pilling, M. J.: Protocol for the development of the Master Chemical Mechanism, MCM v3 (Part A): tropospheric degradation of nonaromatic volatile organic compounds, Atmos. Chem. Phys., 3, 161-180, https://doi.org/10.5194/acp-3-161-2003, 2003.

Schwantes, R. H., Teng, A. P., Nguyen, T. B., Coggon, M. M., Crounse, J. D., St. Clair, J., Zhang, X., Schilling, K. A., Seinfeld, J. H., and Wennberg, P. O.: Isoprene $\mathrm{NO}_{3}$ oxidation products from the $\mathrm{RO}_{2}+\mathrm{HO}_{2}$ pathway, J. Phys. Chem. A, 119, 1015810171, 2015.

Scribano, Y., Goldman, N., Saykally, R. J., and Leforestier, C.: Water dimers in the atmosphere III: Equilibrium constant from a flexible potential, J. Phys. Chem. A., 110, 5411-5419, 2006.

Seinfeld, J. H. and Pandis, S. N.: Atmospheric Chemistry and Physics: From Air Pollution to Climate Change, 2nd ed., John Wiley, Hoboken, N.J., USA, 2006.

Shaw, M. F., Sztáray, B., Whalley, L. K., Heard, D. E., Millet, D. B., Jordan, M. J. T., Osborn, D. L., and Kable, S. H.: Photo-tautomerization of acetaldehyde as a photochemical source of formic acid in the troposphere, Nat. Commun., 9, 2584, https://doi.org/10.1038/s41467-018-04824-2, 2018.

Sheps, L., Rotavera, B., Eskola, A. J., Osborn, D. L., Taatjes, C. A., Au, K., Shallcross, D. E., Khan, M. A. H., and Percival, C. J.: The reaction of Criegee intermediate $\mathrm{CH}_{2} \mathrm{OO}$ with water dimer: primary products and atmospheric impact, Phys. Chem. Chem. Phys., 19, 21970-21979, 2017.
Silva, S. J., Heald, C. L., and Li, M.: Space-based constraints on terrestrial glyoxal production, J. Geophys. Res., 123, 13583-13574, 2018.

Simpson, I. J., Akagi, S. K., Barletta, B., Blake, N. J., Choi, Y., Diskin, G. S., Fried, A., Fuelberg, H. E., Meinardi, S., Rowland, F. S., Vay, S. A., Weinheimer, A. J., Wennberg, P. O., Wiebring, P., Wisthaler, A., Yang, M., Yokelson, R. J., and Blake, D. R.: Boreal forest fire emissions in fresh Canadian smoke plumes: $\mathrm{C}_{1}-\mathrm{C}_{10}$ volatile organic compounds (VOCs), $\mathrm{CO}_{2}, \mathrm{CO}, \mathrm{NO}_{2}$, $\mathrm{NO}, \mathrm{HCN}$ and $\mathrm{CH}_{3} \mathrm{CN}$, Atmos. Chem. Phys., 11, 6445-6463, https://doi.org/10.5194/acp-11-6445-2011, 2011.

Sindelarova, K., Granier, C., Bouarar, I., Guenther, A., Tilmes, S., Stavrakou, T., Müller, J.-F., Kuhn, U., Stefani, P., and Knorr, W.: Global data set of biogenic VOC emissions calculated by the MEGAN model over the last 30 years, Atmos. Chem. Phys., 14, 9317-9341, https://doi.org/10.5194/acp-14-9317-2014, 2014.

Smith, M. C., Chang, C.-H., Chao, W., Lin, L.-C., Takahashi, K., Boering, K. A., and Lin, J. J.: Strong negative temperature dependence of the simplest Criegee Intermediate $\mathrm{CH}_{2} \mathrm{OO}$ reaction with water dimer, J. Phys. Chem. Lett., 6, 2708-2713, 2015.

So, S., Wille, U., and da Silva, G.: Atmospheric chemistry of enols: A theoretical study of the vinyl alcohol $+\mathrm{OH}+\mathrm{O}_{2}$ reaction mechanism, Environ. Sci. Technol., 48, 6694-6701, 2014.

Sofiev, M., Vankevich, R., Ermakova, T., and Hakkarainen, J.: Global mapping of maximum emission heights and resulting vertical profiles of wildfire emissions, Atmos. Chem. Phys., 13, 7039-7052, https://doi.org/10.5194/acp-13-7039-2013, 2013.

Squire, O. J., Archibald, A. T., Griffiths, P. T., Jenkin, M. E., Smith, D., and Pyle, J. A.: Influence of isoprene chemical mechanism on modelled changes in tropospheric ozone due to climate and land use over the 21st century, Atmos. Chem. Phys., 15, 5123-5143, https://doi.org/10.5194/acp-15-5123-2015, 2015.

Stadtler, S., Kühn, T., Schröder, S., Taraborrelli, D., Schultz, M. G., and Kokkola, H.: Isoprene-derived secondary organic aerosol in the global aerosol-chemistry-climate model ECHAM6.3.0-HAM2.3-MOZ1.0, Geosci. Model Dev., 11, 3235-3260, https://doi.org/10.5194/gmd-11-3235-2018, 2018.

Stavrakou, T., Müller, J.-F., De Smedt, I., Van Roozendael, M., van der Werf, G. R., Giglio, L., and Guenther, A.: Evaluating the performance of pyrogenic and biogenic emission inventories against one decade of space-based formaldehyde columns, Atmos. Chem. Phys., 9, 1037-1060, https://doi.org/10.5194/acp-91037-2009, 2009a.

Stavrakou, T., Müller, J.-F., De Smedt, I., Van Roozendael, M., Kanakidou, M., Vrekoussis, M., Wittrock, F., Richter, A., and Burrows, J. P.: The continental source of glyoxal estimated by the synergistic use of spaceborne measurements and inverse modelling, Atmos. Chem. Phys., 9, 8431-8446, https://doi.org/10.5194/acp-9-8431-2009, 2009b.

Stavrakou, T., Peeters, J., and Müller, J.-F.: Improved global modelling of HOx recycling in isoprene oxidation: evaluation against the GABRIEL and INTEX-A aircraft campaign measurements, Atmos. Chem. Phys., 10, 9863-9878, https://doi.org/10.5194/acp-10-9863-2010, 2010.

Stavrakou, T., Guenther, A., Razavi, A., Clarisse, L., Clerbaux, C., Coheur, P.-F., Hurtmans, D., Karagulian, F., De Mazière, M., Vigouroux, C., Amelynck, C., Schoon, N., Laffineur, Q., Heinesch, B., Aubinet, M., Rinsland, C., and Müller, J.-F.: First space-based derivation of the global atmospheric 
methanol emission fluxes, Atmos. Chem. Phys., 11, 4873-4898, https://doi.org/10.5194/acp-11-4873-2011, 2011.

Stavrakou, T., Müller, J.-F., Peeters, J., Razavi, A., Clarisse, L., Clerbaux, C., Coheur, P.-F., Hurtmans, D., De Mazière, M., Vigouroux, C., Deutscher, N. M., Griffith, D. W. T., Jones, N., and Paton-Walsh, C.: Satellite evidence for a large source of formic acid from boreal and tropical forests, Nat. Geosci., 5, 2630, 2012.

Stavrakou, T., Müller, J.-F., Boersma, K. F., van der A, R. J., Kurokawa, J., Ohara, T., and Zhang, Q.: Key chemical $\mathrm{NO}_{x}$ sink uncertainties and how they influence top-down emissions of nitrogen oxides, Atmos. Chem. Phys., 13, 9057-9082, https://doi.org/10.5194/acp-13-9057-2013, 2013.

Stavrakou, T., Müller, J.-F., Bauwens, M., De Smedt, I., Van Roozendael, M., De Mazière, M., Vigouroux, C., Hendrick, F., George, M., Clerbaux, C., Coheur, P.-F., and Guenther, A.: How consistent are top-down hydrocarbon emissions based on formaldehyde observations from GOME-2 and OMI?, Atmos. Chem. Phys., 15, 11861-11884, https://doi.org/10.5194/acp-1511861-2015, 2015.

St. Clair, J. M., Rivera-Rios, J., Crounse, J. D., Knap, H. C., Bates, K. H., Teng, A. P., Jorgensen, S., Kjaergaard, H. G., Keutsch, F. N., and Wennberg, P. O.: Kinetics and products of the reaction of the first-generation isoprene hydroxy hydroperoxide (ISOPOOH) with OH, J. Phys. Chem. A, 120, 1441-1451, 2016.

Surratt, J. D., Chan, A. W. H., Eddingsaas, N. C., Chan, M., Loza, C. L., Kwan, A. J., Hersey, S. P., Flagan, R. C., Wennberg, P. O., and Seinfeld, J. H.: Reactive intermediates revealed in secondary organic aerosol formation from isoprene, P. Nat. Acad. Sci. USA, 107, 6640-6645, 2010.

Tadic, J., Juranic, I., and Moortgat, G. K.: Pressure dependence of the photooxidation of selected carbonyl compounds in air: nbutanal and n-pentanal, J. Photochem. Photobiol. A, 143, 169179, 2001a.

Tadic, J., Juranic, I., and Moortgat, G. K.: Photooxidation of nhexanal in air, Molecules, 6, 287-299, 2001b.

Taraborrelli, D., Lawrence, M. G., Crowley, J. N., Dillon, T. J., Gromov, S., Grosz, C. B. M., Vereecken, L., and Lelieveld, J.: Hydroxyl radical buffered by isoprene oxidation over tropical forests, Nat. Geosci., 5, 190-193, 2012.

Teng, A. P., Crounse, J. D., Lee, L., St. Clair, J. M., Cohen, R. C., and Wennberg, P. O.: Hydroxy nitrate production in the $\mathrm{OH}-$ initiated oxidation of alkenes, Atmos. Chem. Phys., 15, 42974316, https://doi.org/10.5194/acp-15-4297-2015, 2015.

Teng, A. P., Crounse, J. D., and Wennberg, P. O.: Isoprene peroxy radical dynamics, J. Am. Chem. Soc., 139, 5367-5377, 2017.

Thuner, L. P., Rea, G., and Wenger, J. C.: Photolysis of butenedial and 4-oxopent-2-enal, in The European Photoreactor EUPHORE 4th Report 2001, ed. I. Barnes, Bergische Universitat Wuppertal, Wuppertal, Germany, 41-46, 2003.

Toon, O. B., Maring, H., Dibb, J., Ferrare, R., Jacob, D. J., Jensen, E. J., J., Z., Mace, G. G., Pan, L. L., Pfister, L., Rosenlof, K. H., Redemann, J., Reid, J. S., Singh, H. B., Yokelson, R., Minnis, P., Chen, G., Jucks, K. W., and Pszenny, A.: Planning, implementation and scientific goals of the Studies of Emissions and Atmospheric Composition, Clouds and Climate Coupling by Regional SSurvey (SEAC4RS) field mission, J. Geophys. Res., 121, 4967-5009, 2016.
Trainer, M., Williams, E. J., Parrish, D. D., Buhr, M. P., Allwine, E. J., Westberg, H. H., Fehsenfeld, F. C., and Liu, S. C.: Models and observations of the impact of natural hydrocarbons on rural ozone, Nature, 329, 705-707, 1987.

Travis, K. R., Jacob, D. J., Fisher, J. A., Kim, P. S., Marais, E. A., Zhu, L., Yu, K., Miller, C. C., Yantosca, R. M., Sulprizio, M P., Thompson, A. M., Wennberg, P. O., Crounse, J. D., St. Clair, J. M., Cohen, R. C., Laughner, J. L., Dibb, J. E., Hall, S. R., Ullmann, K., Wolfe, G. M., Pollack, I. B., Peischl, J., Neuman, J. A., and Zhou, X.: Why do models overestimate surface ozone in the Southeast United States?, Atmos. Chem. Phys., 16, 1356113577, https://doi.org/10.5194/acp-16-13561-2016, 2016.

Tuazon, E. C. and Atkinson, R. A.: A product study of the gasphase reaction of methyl vinyl ketone with the $\mathrm{OH}$ radical in the presence of $\mathrm{NO}_{x}$, Int. J. Chem. Kinet., 21, 1141-1152, 1989.

van der Werf, G. R., Randerson, J. T., Giglio, L., van Leeuwen, T. T., Chen, Y., Rogers, B. M., Mu, M., van Marle, M. J. E., Morton, D. C., Collatz, G. J., Yokelson, R. J., and Kasibhatla, P. S.: Global fire emissions estimates during 1997-2016, Earth Syst. Sci. Data, 9, 697-720, https://doi.org/10.5194/essd-9-697-2017, 2017.

Vasquez, K. T., Allen, H. M., Crounse, J. D., Praske, E., Xu, L., Noelscher, A. C., and Wennberg, P. O.: Low-pressure gas chromatography with chemical ionization mass spectrometry for quantification of multifunctional organic compounds in the atmosphere, Atmos. Meas. Tech., 11, 6815-6832, https://doi.org/10.5194/amt-11-6815-2018, 2018.

Vereecken, L. and Peeters, J.: Decomposition of substituted alkoxy radicals - part I: a generalized structure-activity relationship for reaction barrier heights, Phys. Chem. Chem. Phys., 11, 90629074, 2009.

Vu, N. D., Khamaganov, V., Nguyen, V. S., Carl, S. A., and Peeters, J.: Absolute rate coefficient of the gas-phase reaction between hydroxyl radical $(\mathrm{OH})$ and hydroxyacetone: Investigating the effects of temperature and pressure, J. Phys. Chem. A, 117, 1220812215, 2013.

Wennberg, P. O., Bates, K. H., Crounse, J. D., Dodson, L. G., McVay, R., Mertens, L. A., Nguyen, T. B., Praske, E., Schwantes, R. H., Smarte, M. D., St Clair, J. M., Teng, A. P., Zhang, X., and Seinfeld, J. H.: Gas-phase reactions of isoprene and its major oxidation products, Chem. Rev., 118, 3337-3390, https://doi.org/10.1021/acs.chemrev.7b00439, 2018.

Wolfe, G. M., Crounse, J. D., Parrish, J. D., St Clair, J. M., Beaver, M. R., Paulot, F., Yoon, T. P., Wennberg, P. O., and Keutsch, F. N.: Photolysis, $\mathrm{OH}$ reactivity and ozone reactivity of a proxy for isoprene-derived hydroperoxyenals (HPALDs), Phys. Chem. Chem. Phys., 14, 7276-7286, 2012.

Wolfe, G. M., Hanisco, T. F., Arkinson, H. L., Bui, T. P., Crounse, J. D., Dean-Day, J., Goldstein, A., Guenther, A., Hall, S. R., Huey, G., Jacob, D. J., Karl, T., Kim, P. S., Liu, X., Marvin, M. R., Mikoviny, T., Misztal, P., Nguyen, T. B., Peischl, J., Pollack, I., Ryerson, T., St. Clair, J. M., Teng, A., Travis, K. R., Ullmann, K., Wennberg, P. O., and Wisthaler, A.: Quantifying sources and sinks of reactive gases in the lower atmosphere using airborne flux observations, Geophys. Res. Lett., 42, 8231-8240, 2015.

Xiong, F., Borca, C. H., Slipchenko, L. V., and Shepson, P. B.: Photochemical degradation of isoprene-derived 4,1-nitrooxy enal, Atmos. Chem. Phys., 16, 5595-5610, https://doi.org/10.5194/acp-16-5595-2016, 2016. 
Yan, C., Kocevska, S., and Krasnoperov, L. N.: Kinetics of the reaction of $\mathrm{CH}_{3} \mathrm{O}_{2}$ radicals with $\mathrm{OH}$ studied over the $292-526 \mathrm{~K}$ temperature range, J. Phys. Chem. A, 120, 6111-6121, 2016.
Yoon M.-C., Choi Y. S., and Kim, S. K.: The OH production from the transition of acetylacetone, Chem. Phys. Lett., 300, 207-212, 1999. 